\title{
HOCHSCHILD COHOMOLOGY RING FOR SELF-INJECTIVE ALGEBRAS OF TREE CLASS $E_{6}$. II.
}

\author{
MARIYA KACHALOVA
}

\begin{abstract}
We describe the Hochschild cohomology ring for a family of self-injective algebras of tree class $E_{6}$ in terms of generators and relations. Together with the results of the previous paper, this gives a complete description of the Hochschild cohomology ring for a self-injective algebras of tree class $E_{6}$.
\end{abstract}

\section{CONTEnTs}

1. Introduction

2. Statement of the main results

3. Bimodule resolution

4. The additive structure of $\mathrm{HH}^{*}(R)$

5. Generators of $\mathrm{HH}^{*}(R)$

6. $\Omega$-shifts of generators of the algebra $\mathrm{HH}^{*}(R)$

7. Multiplications in $\mathrm{HH}^{*}(R)$

References

\section{INTRODUCTION}

Consider a self-injective basic algebra of finite representation type over an algebraically closed field. According to Riedtmann's classification, the stable $A R$-quiver of such an algebra can be described with the help of an associated tree, which must be congruent with one of the Dynkin diagrams $A_{n}, D_{n}, E_{6}, E_{7}$, or $E_{8}$ (see [1]). The complete description of the Hochschild cohomology ring was obtained for an algebras of the types $A_{n}$ and $D_{n}$, see [2 [5] (type $A_{n}$ ) and [6 11] (type $D_{n}$ ). For one of the two self-injective algebras of tree class $E_{6}$ Hochschild cohomology ring was obtained in [12].

In this paper we consider the second part of algebras of tree class $E_{6}$. Any algebra of the class $E_{6}$ is derived equivalent to the path algebra for some quiver with relations. Namely, let $\mathcal{Q}_{s}$ $(s \in \mathbb{N})$ is the following quiver: 


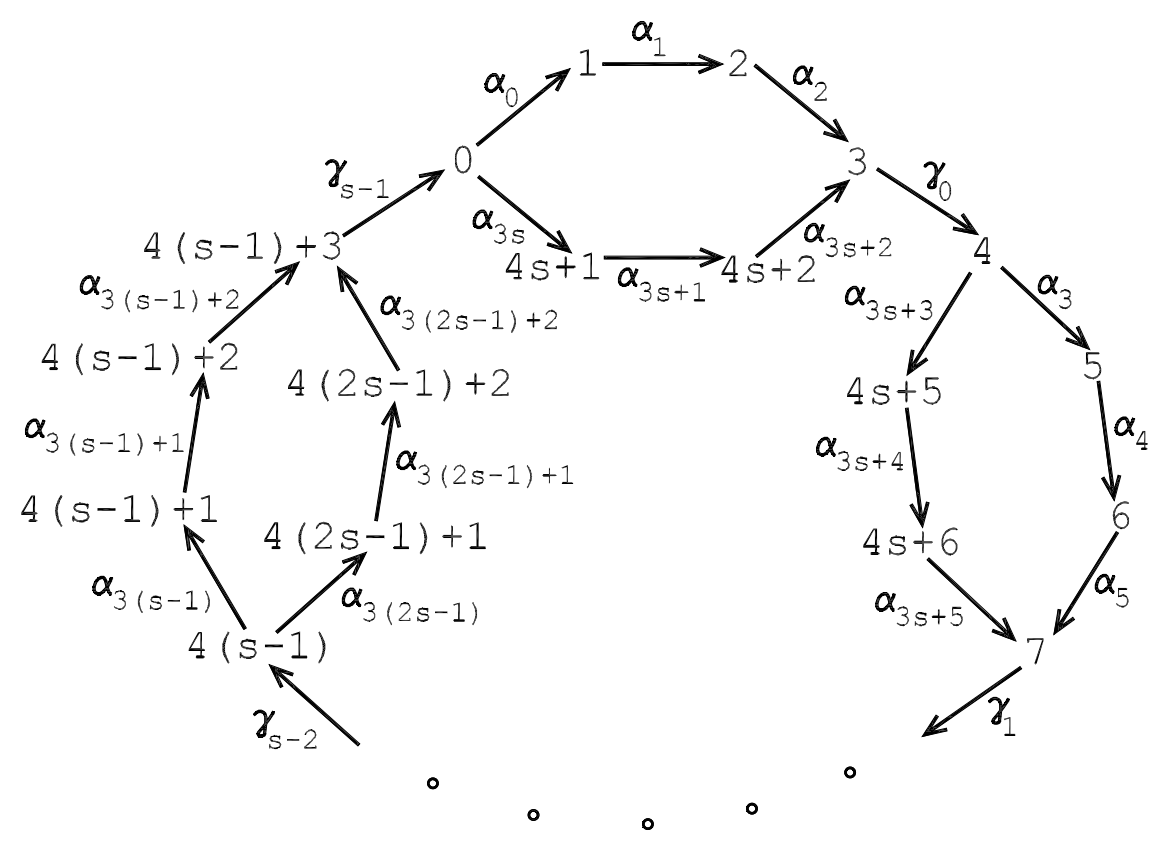

Then any algebra of the class $E_{6}$ is derived equivalent to one of the two following algebras:

1) $R_{s}=K\left[\mathcal{Q}_{s}\right] / I$, where $K$ is a field, and $I$ is the ideal in the path algebra $K\left[\mathcal{Q}_{s}\right]$ of the quiver $\mathcal{Q}_{s}$, generated by

a) all the paths of length 5 ;

b) the expressions of the form:

$$
\begin{gathered}
\alpha_{3 t+2} \alpha_{3 t+1} \alpha_{3 t}-\alpha_{3(t+s)+2} \alpha_{3(t+s)+1} \alpha_{3(t+s)}, \\
\alpha_{3 t} \gamma_{t-1} \alpha_{3(t+s)-1}, t \in[0,2 s-1] \backslash\{0, s\}, \\
\alpha_{0} \gamma_{s-1} \alpha_{3(2 s-1)+2}, \alpha_{3 s} \gamma_{s-1} \alpha_{3(s-1)+2} .
\end{gathered}
$$

2) $R_{s}^{\prime}=K\left[\mathcal{Q}_{s}\right] / I^{\prime}$, where $K$ is a field, and $I^{\prime}$ is the ideal in the path algebra $K\left[\mathcal{Q}_{s}\right]$ of the quiver $\mathcal{Q}$, generated by

a) all the paths of length 5 ;

b) the expressions of the form:

$$
\begin{gathered}
\alpha_{3 t+2} \alpha_{3 t+1} \alpha_{3 t}-\alpha_{3(t+s)+2} \alpha_{3(t+s)+1} \alpha_{3(t+s)}, \\
\alpha_{3 t} \gamma_{t-1} \alpha_{3(t+s)-1} .
\end{gathered}
$$

Henceforth we will often omit indexes in arrows $\alpha_{i}$ and $\gamma_{i}$ as long as subscripts are clear from the context.

The present paper is dedicated to the study of Hochschild cohomology ring structure for algebra $R_{s}^{\prime}$. We obtain the ring structure in terms of generators and relations. In studies of the structure of cohomology ring we will construct the bimodule resolution of $R_{s}^{\prime}$, which could be seen as a whole result.

\section{Statement of the main Results}

In what follows, we assume $n=6$.

Let $\mathrm{HH}^{t}(R)$ is the $t$ th group of the Hochschild cohomology ring of $R$ with coefficients in $R$. Let $\ell$ be the aliquot, and $r$ be the residue of division of $t$ by 11, $m$ be the aliquot of division of $r$ by 2 . 
HOCHSCHILD COHOMOLOGY RING FOR SELF-INJECTIVE ALGEBRAS OF TREE CLASS $E_{6}$. II.

Consider the case of $s>1$. To describe Hochschild cohomology ring of algebra $R_{s}^{\prime}$ we must introduce the following conditions on an arbitrary degree $t$ :

(1) $r=0, \ell(n+s)+m \equiv 0(2 s), \ell \vdots 2$ or $\operatorname{char} K=2$;

(2) $r=0, \ell(n+s)+m \equiv s+1(2 s), \ell \vdots 2, \operatorname{char} K=3$;

(3) $r=1, \ell(n+s)+m \equiv 0(2 s), \ell \vdots 2$ or $\operatorname{char} K=2$;

(4) $r=1, \ell(n+s)+m \equiv s(2 s), \ell \% 2$ or char $K=2$;

(5) $r=2, \ell(n+s)+m \equiv s+1(2 s), \ell \% 2$ or char $K=2$;

(6) $r=3, \ell(n+s)+m \equiv 0(2 s)$;

(7) $r=3, \ell(n+s)+m \equiv s(2 s)$, char $K=2$;

(8) $r=4, \ell(n+s)+m \equiv s+1(2 s)$, char $K=2$;

(9) $r=4, \ell(n+s)+m \equiv s(2 s), \ell \% 2$, char $K=3$;

(10) $r=4, \ell(n+s)+m \equiv 1(2 s)$;

(11) $r=5, \ell(n+s)+m \equiv 0(2 s), \ell \vdots 2, \operatorname{char} K=3$;

(12) $r=5, \ell(n+s)+m \equiv s(2 s), \ell \% 2$, char $K=3$;

(13) $r=6, \ell(n+s)+m \equiv 0(2 s)$, char $K=2$;

(14) $r=6, \ell(n+s)+m \equiv 1(2 s), \ell \vdots 2, \operatorname{char} K=3$;

(15) $r=6, \ell(n+s)+m \equiv s(2 s)$;

(16) $r=7, \ell(n+s)+m \equiv 0(2 s)$, char $K=2$;

(17) $r=7, \ell(n+s)+m \equiv s(2 s)$;

(18) $r=8, \ell(n+s)+m \equiv 0(2 s), \ell \vdots 2$ or $\operatorname{char} K=2$;

(19) $r=9, \ell(n+s)+m \equiv 0(2 s), \ell \vdots 2$ or $\operatorname{char} K=2$;

(20) $r=9, \ell(n+s)+m \equiv s(2 s), \ell \% 2$ or char $K=2$;

(21) $r=10, \ell(n+s)+m \equiv s+1(2 s), \ell \% 2$ or char $K=2$;

(22) $r=10, \ell(n+s)+m \equiv 0(2 s), \ell \% 2$, char $K=3$.

Let

$$
M_{0}=\frac{2 s}{\operatorname{gcd}(n+s, 2 s)}, \quad M= \begin{cases}11 M_{0}, & \text { char } K=2 \text { or } M_{0} \vdots 4 \\ 22 M_{0} & \text { otherwise }\end{cases}
$$

Remark 1 . We will prove in paragraph 3 that the minimal period of bimodule resolution of $R_{s}^{\prime}$ is $M$.

Let $\left\{t_{1, i}, \ldots, t_{\alpha_{i}, i}\right\}$ be a set of all degrees $t$, that satisfy the conditions of item $i$ from the above list, and such that $0 \leqslant t_{j, i}<M\left(j=1, \ldots, \alpha_{i}\right)$. Consider the set

$$
\mathcal{X}=\bigcup_{i=1}^{22}\left\{X_{t_{j, i}}^{(i)}\right\}_{j=1}^{\alpha_{i}} \cup\{T\}
$$

and define a graduation of polynomial ring $K[\mathcal{X}]$ such that

$$
\operatorname{deg} X_{t_{j, i}}^{(i)}=t_{j, i} \text { for all } i=1, \ldots, 22 \text { and } j=1, \ldots, \alpha_{i} \text {; }
$$

$$
\operatorname{deg} T=M \text {. }
$$


Remark 2. Hereafter we shall use simplified denotation $X^{(i)}$ instead of $X_{t_{j, i}}^{(i)}$, since lower indexes are clear from context.

\section{Denotation.}

$$
\tilde{X}^{(i)}=\left\{\begin{array}{l}
X^{(i)}, \quad \operatorname{deg} \widetilde{X}^{(i)}<\operatorname{deg} T \\
T X^{(i)}, \quad \text { otherwise. }
\end{array}\right.
$$

Define a graduate $K$-algebra $\mathcal{A}=K[\mathcal{X}] / I$, where $I$ is the ideal generated by homogeneous elements corresponding to the following relations.

$$
\begin{aligned}
& X^{(3)} X^{(2)}=X^{(3)} X^{(3)}=X^{(3)} X^{(5)}=X^{(3)} X^{(7)}=X^{(3)} X^{(8)}=0 ; \\
& X^{(3)} X^{(9)}=X^{(3)} X^{(10)}=X^{(3)} X^{(11)}=X^{(3)} X^{(12)}=X^{(3)} X^{(13)}=0 ; \\
& X^{(3)} X^{(14)}=X^{(3)} X^{(16)}=X^{(3)} X^{(17)}=X^{(3)} X^{(19)}=X^{(3)} X^{(21)}=X^{(3)} X^{(21)}=0 ; \\
& X^{(3)} X^{(1)}=\tilde{X}^{(3)}, \quad X^{(3)} X^{(4)}=\tilde{X}^{(5)}, \quad X^{(3)} X^{(6)}=\widetilde{X}^{(10)} ; \\
& X^{(3)} X^{(15)}=\tilde{X}^{(17)}, \quad X^{(3)} X^{(18)}=\tilde{X}^{(19)}, \quad X^{(3)} X^{(20)}=\tilde{X}^{(21)} ;
\end{aligned}
$$

$$
\begin{aligned}
X^{(4)} X^{(6)} & = \begin{cases}\tilde{X}^{(8)}, & \text { char } K=2, \\
0, & \text { otherwise }\end{cases} \\
X^{(6)} X^{(6)} & = \begin{cases}-s \widetilde{X}^{(14)}, & \text { char } K=3, \\
0, & \text { otherwise; }\end{cases} \\
X^{(4)} X^{(15)} & = \begin{cases}\tilde{X}^{(16)}, & \text { char } K=2, \\
0, & \text { otherwise; }\end{cases} \\
X^{(6)} X^{(18)} & = \begin{cases}-s \tilde{X}^{(2)}, & \text { char } K=3, \\
0, & \text { otherwise; }\end{cases} \\
X^{(15)} X^{(20)} & = \begin{cases}\tilde{X}^{(8)}, & \text { char } K=2, \\
0, & \text { otherwise; }\end{cases} \\
X^{(18)} X^{(18)} & = \begin{cases}s \widetilde{X}^{(12)}, & \text { char } K=3, \\
0, & \text { otherwise; }\end{cases} \\
X^{(18)} X^{(20)} & = \begin{cases}s \widetilde{X}^{(14)}, & \text { char } K=3, \\
0, & \text { otherwise }\end{cases}
\end{aligned}
$$

Describe the rest relations as a tables (numbers $(\mathrm{r} 1)-(\mathrm{r} 7)$ in tables cells are the number of relation that defines a multiplication of the following elements). 
HOCHSCHILD COHOMOLOGY RING FOR SELF-INJECTIVE ALGEBRAS OF TREE CLASS $E_{6}$. II. 5

\begin{tabular}{c|c|c|c|c|c|c|c|c} 
& $X^{(1)}$ & $X^{(2)}$ & $X^{(4)}$ & $X^{(6)}$ & $X^{(7)}$ & $X^{(8)}$ & $X^{(9)}$ & $X^{(11)}$ \\
\hline$X^{(1)}$ & $X^{(1)}$ & $X^{(2)}$ & $X^{(4)}$ & $X^{(6)}$ & $X^{(7)}$ & $X^{(8)}$ & $X^{(9)}$ & $X^{(11)}$ \\
\hline$X^{(2)}$ & & 0 & 0 & 0 & 0 & 0 & $-X^{(10)}$ & 0 \\
\hline$X^{(4)}$ & & & 0 & $(1)$ & $-X^{(10)}$ & 0 & $X^{(11)}$ & 0 \\
\hline$X^{(6)}$ & & & & $(2)$ & 0 & 0 & $s X^{(17)}$ & 0 \\
\hline$X^{(7)}$ & & & & & 0 & 0 & 0 & 0 \\
\hline$X^{(8)}$ & & & & & & 0 & 0 & 0 \\
\hline$X^{(9)}$ & & & & & & & 0 & 0 \\
\hline$X^{(11)}$ & & & & & & & & 0
\end{tabular}

\begin{tabular}{c|c|c|c|c|c|c|c|c} 
& $X^{(12)}$ & $X^{(13)}$ & $X^{(14)}$ & $X^{(15)}$ & $X^{(16)}$ & $X^{(18)}$ & $X^{(20)}$ & $X^{(22)}$ \\
\hline$X^{(1)}$ & $X^{(12)}$ & $X^{(13)}$ & $X^{(14)}$ & $X^{(15)}$ & $X^{(16)}$ & $X^{(18)}$ & $X^{(20)}$ & $X^{(22)}$ \\
\hline$X^{(2)}$ & 0 & 0 & 0 & $X^{(14)}$ & 0 & 0 & 0 & $-X^{(21)}$ \\
\hline$X^{(4)}$ & $X^{(14)}$ & $X^{(17)}$ & 0 & $(3)$ & 0 & $X^{(20)}$ & 0 & 0 \\
\hline$X^{(6)}$ & 0 & $X^{(19)}$ & 0 & $-X^{(20)}$ & 0 & $(4)$ & 0 & $s X^{(5)}$ \\
\hline$X^{(7)}$ & 0 & 0 & 0 & $X^{(19)}$ & $X^{(21)}$ & 0 & 0 & 0 \\
\hline$X^{(8)}$ & 0 & $X^{(21)}$ & 0 & 0 & 0 & 0 & 0 & 0 \\
\hline$X^{(9)}$ & $X^{(19)}$ & 0 & $X^{(21)}$ & $-X^{(22)}$ & 0 & $s X^{(3)}$ & $s X^{(5)}$ & 0 \\
\hline$X^{(11)}$ & $X^{(21)}$ & 0 & 0 & 0 & 0 & $s X^{(5)}$ & 0 & 0
\end{tabular}

\begin{tabular}{c|c|c|c|c|c|c|c|c} 
& $X^{(12)}$ & $X^{(13)}$ & $X^{(14)}$ & $X^{(15)}$ & $X^{(16)}$ & $X^{(18)}$ & $X^{(20)}$ & $X^{(22)}$ \\
\hline$X^{(12)}$ & 0 & 0 & 0 & $-X^{(2)}$ & 0 & 0 & 0 & $-X^{(10)}$ \\
\hline$X^{(13)}$ & & 0 & 0 & $X^{(3)}$ & $X^{(5)}$ & $X^{(7)}$ & $X^{(10)}$ & 0 \\
\hline$X^{(14)}$ & & & 0 & 0 & 0 & 0 & 0 & 0 \\
\hline$X^{(15)}$ & & & & $-X^{(4)}$ & 0 & $X^{(6)}$ & $(5)$ & $X^{(11)}$ \\
\hline$X^{(16)}$ & & & & & 0 & $X^{(8)}$ & 0 & 0 \\
\hline$X^{(18)}$ & & & & & & $(6)$ & $(7)$ & $-s X^{(17)}$ \\
\hline$X^{(20)}$ & & & & & & & 0 & 0 \\
\hline$X^{(22)}$ & & & & & & & & 0
\end{tabular}

Theorem 1. Let $s>1, R=R_{s}^{\prime}$ is algebra of the type $E_{6}$. Then the Hochschild cohomology ring $\mathrm{HH}^{*}(R)$ is isomorphic to $\mathcal{A}$ as a graded $K$-algebra.

Consider the case of $s=1$.

Let us introduce the set

$$
\mathcal{X}^{\prime}=\left\{\begin{array}{l}
\mathcal{X} \cup\left\{X_{0}^{(23)}, X_{0}^{(24)}\right\}, \quad \operatorname{char} K \neq 3 \\
\mathcal{X} \cup\left\{X_{0}^{(24)}\right\}, \quad \operatorname{char} K=3
\end{array}\right.
$$


and define a graduation of polynomial ring $K\left[\mathcal{X}^{\prime}\right]$ such that

$$
\begin{aligned}
& \operatorname{deg} X_{t_{j, i}}^{(i)}=t_{j, i} \text { for all } i=1, \ldots, 22 \text { and } j=1, \ldots, \alpha_{i} ; \\
& \operatorname{deg} T=M(\text { similar to (回) }) ; \\
& \operatorname{deg} X_{0}^{(23)}=\operatorname{deg} X_{0}^{(24)}=0 .
\end{aligned}
$$

Define a graduate $K$-algebra $\mathcal{A}^{\prime}=K\left[\mathcal{X}^{\prime}\right] / I^{\prime}$, where $I^{\prime}$ is the ideal generated by homogeneous elements corresponding to the relations described in the case of $s>1$, and by the following relations:

$$
\begin{aligned}
X^{(1)} X^{(23)} & = \begin{cases}\tilde{X}^{(23)}, & t_{1}=0 ; \\
0, & \text { otherwise; }\end{cases} \\
X^{(1)} X^{(24)} & = \begin{cases}\widetilde{X}^{(24)}, & t_{1}=0 ; \\
\tilde{X}^{(2)}, & t_{1}>0 \text { and char } K=3 ; \\
0, & \text { otherwise; }\end{cases} \\
X^{(9)} X^{(24)} & =-\widetilde{X}^{(10)} ; \\
X^{(15)} X^{(24)} & = \begin{cases}\widetilde{X}^{(14)}, & \text { char } K=3 ; \\
0, & \text { otherwise; }\end{cases} \\
X^{(22)} X^{(24)} & =-\widetilde{X}^{(21)} ; \quad j \in\{2,24] \backslash\{9,15,22\}, \quad i \in\{\},
\end{aligned}
$$

where $t_{1}$ denotes a degree of the element $X^{(1)}$.

Theorem 2. Let $s=1, R=R_{1}^{\prime}$ is algebra of the type $E_{6}$. Then the Hochschild cohomology ring $\mathrm{HH}^{*}(R)$ is isomorphic to $\mathcal{A}^{\prime}$ as a graded $K$-algebra.

Remark 3. From the descriptions of rings $\mathrm{HH}^{*}(R)$ given in theorems 1 and 2 it implies, in particular, that they are commutative.

\section{Bimodule Resolution}

We will construct the minimal projective bimodule resolution of the $R$ in the following form:

$$
\cdots \longrightarrow Q_{3} \stackrel{d_{2}}{\longrightarrow} Q_{2} \stackrel{d_{1}}{\longrightarrow} Q_{1} \stackrel{d_{0}}{\longrightarrow} Q_{0} \stackrel{\varepsilon}{\longrightarrow} R \longrightarrow 0
$$

Let $\Lambda$ be an enveloping algebra of algebra $R$. Then $R$-R-bimodules can be considered as left $\Lambda$-modules.

\section{Denotations.}

(1) Let $e_{i}, i \in \mathbb{Z}_{(n+2) s}=\{0,1, \ldots,(n+2) s-1\}$, be the idempotents of the algebra $K\left[\mathcal{Q}_{s}\right]$, that correspond to the vertices of the quiver $\mathcal{Q}_{s}$.

(2) Denote by $P_{i, j}=R\left(e_{i} \otimes e_{j}\right) R=\Lambda\left(e_{i} \otimes e_{j}\right), i, j \in \mathbb{Z}_{(n+2) s}$. Note that the modules $P_{i, j}$, forms the full set of the (pairwise non-isomorphic by) indecomposable projective $\Lambda$-modules.

(3) For $a \in \mathbb{Z}, t \in \mathbb{N}$ we denote the smallest nonnegative deduction of $a$ modulo $t$ with $(a)_{t}$ (in particular, $\left.0 \leqslant(a)_{t} \leqslant t-1\right)$. 
HOCHSCHILD COHOMOLOGY RING FOR SELF-INJECTIVE ALGEBRAS OF TREE CLASS $E_{6}$. II.

Let $R=R_{s}^{\prime}$. We introduce an automorphism $\sigma: R \rightarrow R$, which is mapping as follows:

$$
\begin{gathered}
\sigma\left(e_{i}\right)=e_{4(n+s)+i} \\
\sigma\left(\gamma_{i}\right)=\left\{\begin{array}{ll}
\gamma_{i+n}, \quad(i)_{s}=s-1 ; \\
-\gamma_{i+n}, \quad(i)_{s}<s-1,
\end{array} \quad \sigma\left(\alpha_{i}\right)= \begin{cases}-\alpha_{3(n+s)+i}, \quad(i)_{3}=0, \quad(i)_{6 s}<3 s ; \\
\alpha_{3(n+s)+i}, \quad(i)_{3}=0, \quad(i)_{6 s} \geqslant 3 s ; \\
-\alpha_{3(n+s)+i}, & (i)_{3}=1 ; \\
-\alpha_{3(n+s)+i}, & (i)_{3}=2, \quad(i)_{6 s} \geqslant 3 s ; \\
\alpha_{3(n+s)+i}, & (i)_{3}=2, \quad(i)_{6 s}<3 s\end{cases} \right.
\end{gathered}
$$

Define the helper functions $f: \mathbb{Z} \times \mathbb{Z} \rightarrow \mathbb{Z}$ and $h: \mathbb{Z} \times \mathbb{Z} \rightarrow \mathbb{Z}$, which act in the following way:

$$
f(x, y)=\left\{\begin{array}{ll}
1, & x=y ; \\
0, & x \neq y,
\end{array} \quad h(x, y)= \begin{cases}1, & x \vdots 2, x<y \\
0, & x \% 2, x<y \\
1, & x \% 2, x \geqslant y \\
0, & x \vdots 2, x \geqslant y\end{cases}\right.
$$

Introduce $Q_{r}(r \leqslant 10)$. Let $m$ be the aliquot of division of $r$ by 2 for considered degree $r$. We have

$$
\begin{aligned}
Q_{2 m} & =\bigoplus_{r=0}^{s-1} Q_{2 m, r}^{\prime}, \quad 0 \leqslant m \leqslant n-1, \\
Q_{2 m+1} & =\bigoplus_{r=0}^{s-1} Q_{2 m+1, r}^{\prime}, \quad 0 \leqslant m \leqslant n-2,
\end{aligned}
$$

where

$$
\begin{aligned}
Q_{2 m, r}^{\prime}=\left(\bigoplus_{i=0}^{f(m, 2)} P_{4(r+m)-1+h(m, 2)+i, 4 r}\right) & \\
& \oplus \bigoplus_{i=0}^{f(m, 3)} \bigoplus_{j=0}^{1} P_{4(r+m+j s+f(m, 2) s+f(m, 5) s)+2-h(m, 3)+i(4 s+1), 4(r+j s)+1} \\
\oplus \bigoplus_{i=0}^{f(m, 2)} \bigoplus_{j=0}^{1} P_{(r+m+j s+f(m, 1) s+f(m, 4) s+f(m, 5) s)+1+h(m, 2)+i(4 s+1), 4(r+j s)+2} & \oplus\left(\bigoplus_{i=0}^{f(m, 3)} P_{4(r+m+1)-h(m, 3)+i, 4 r+3}\right) ;
\end{aligned}
$$




$$
\begin{aligned}
& Q_{2 m+1, r}^{\prime}=\left(\bigoplus_{i=0}^{1-f(m, 4)} P_{4(r+m)+1+h(m, 0)+2 f(m, 4)+4 s i, 4 r}\right) \\
& \oplus \bigoplus_{j=0}^{1} P_{4(r+m+1+j s)-h(m, 0)-2 f(m, 0), 4(r+j s)+1}^{1} \bigoplus_{j=0} P_{4(r+m+1+j s+f(m, 4) s)-h(m, 5)+2 f(m, 4), 4(r+j s)+2} \\
& \oplus\left(\bigoplus_{i=0}^{1-f(m, 0)} P_{4(r+m+1)+1+h(m, 5)-2 f(m, 0)+4 s i, 4 r+3}\right)
\end{aligned}
$$

Now we shall describe differentials $d_{r}$ for $r \leqslant 10$. Since $Q_{i}$ are direct sums, their elements can be concerned as column vectors, hence differentials can be described as matrixes (which are being multiplied by column vectors from the right). Now let us describe the matrixes of differentials componentwisely.

Remark 4. Numeration of lines and columns always starts with zero.

\section{Denotations.}

(1) Denote by $w_{i \rightarrow j}$ the way that starts in $i$ th vertex and ends in $j$ th.

(2) Fot $j$ th column of differential matrix let $j_{2}$ be the aliquot and $i_{2}$ be the residue of division of $j$ by $s$.

Define the helper functions $f_{0}: \mathbb{Z} \times \mathbb{Z} \rightarrow \mathbb{Z}, f_{1}: \mathbb{Z} \times \mathbb{Z} \rightarrow \mathbb{Z}$ and $f_{2}: \mathbb{Z} \times \mathbb{Z} \rightarrow \mathbb{Z}$, which act in the following way:

$$
f_{0}(x, y)=\left\{\begin{array}{ll}
1, & x<y ; \\
0 & x \geqslant y,
\end{array} \quad f_{1}(x, y)=\left\{\begin{array}{ll}
1, & x<y ; \\
-1 & x \geqslant y,
\end{array} \quad f_{2}(x, y)= \begin{cases}1, & x=y \\
-1 & x \neq y\end{cases}\right.\right.
$$

If $0 \leqslant j<2 s$, then

\section{Description of the $d_{0}$}

$$
d_{0}: Q_{1} \rightarrow Q_{0}-\text { is an }(7 s \times 6 s) \text { matrix. }
$$

$$
\left(d_{0}\right)_{i j}= \begin{cases}w_{4(j+m) \rightarrow 4(j+m)+1} \otimes e_{4 j}, & i=(j)_{s} \\ -e_{4(j+m)+1} \otimes w_{4 j \rightarrow 4 j+1}, & i=j+s \\ 0 \quad \text { otherwise. } & \end{cases}
$$

If $2 s \leqslant j<4 s$, then

$$
\left(d_{0}\right)_{i j}=\left\{\begin{array}{l}
w_{4(j+m)+1 \rightarrow 4(j+m)+2} \otimes e_{4 j+1}, \quad i=j-s \\
-e_{4(j+m)+2} \otimes w_{4 j+1 \rightarrow 4 j+2}, \quad i=j+s \\
0 \quad \text { otherwise. }
\end{array}\right.
$$


If $4 s \leqslant j<6 s$, then

$$
\left(d_{0}\right)_{i j}=\left\{\begin{array}{l}
w_{4(j+m)+2 \rightarrow 4(j+m)+3} \otimes e_{4 j+2}, \quad i=j-s \\
-e_{4(j+m)+3} \otimes w_{4 j+2 \rightarrow 4 j+3}, \quad i=(j)_{s}+5 s \\
0 \quad \text { otherwise. }
\end{array}\right.
$$

If $6 s \leqslant j<7 s$, then

$$
\left(d_{0}\right)_{i j}= \begin{cases}-e_{4(j+m+1)} \otimes w_{4 j+3 \rightarrow 4(j+1)}, & i=(j+1)_{s} \\ w_{4(j+m)+3 \rightarrow 4(j+m+1)} \otimes e_{4 j+3}, & i=j-s \\ 0 \quad \text { otherwise. } & \end{cases}
$$

If $0 \leqslant j<s$, then

\section{Description of the $d_{1}$}

$$
d_{1}: Q_{2} \rightarrow Q_{1}-\text { is an }(6 s \times 7 s) \text { matrix. }
$$

$$
\left(d_{1}\right)_{i j}=\left\{\begin{array}{l}
w_{4(j+m)+1+j_{1} \rightarrow 4(j+m)+3} \otimes w_{4 j \rightarrow 4 j+j_{1}}, \quad i=j+2 j_{1} s, \quad 0 \leqslant j_{1} \leqslant 2 \\
-w_{4(j+m+s)+1+j_{1} \rightarrow 4(j+m)+3} \otimes w_{4 j \rightarrow 4(j+s)+j_{1}}, \quad i=j+\left(2 j_{1}+1\right) s, 0 \leqslant j_{1} \leqslant 2 \\
0 \quad \text { otherwise. }
\end{array}\right.
$$

If $s \leqslant j<3 s$, then

$$
\left(d_{1}\right)_{i j}=\left\{\begin{array}{l}
w_{4(j+m+s+1)+1 \rightarrow 4(j+m+s+1)+2} \otimes w_{4(j+s)+1 \rightarrow 4(j+1)}, \quad i=j-s+1, j<2 s \\
w_{4(j+m+s+1)+1 \rightarrow 4(j+m+s+1)+2} \otimes w_{4(j+s)+1 \rightarrow 4(j+1)}, \quad i=(j+s+1)_{2 s}, j \geqslant 2 s \\
w_{4(j+m+s)+2 \rightarrow 4(j+m+s+1)+2} \otimes e_{4(j+s)+1}, \quad i=j+s ; \\
e_{4(j+m+s+1)+2} \otimes w_{4(j+s)+1 \rightarrow 4(j+s+1)+1}, \quad i=j+s+1, j<2 s ; \\
e_{4(j+m+s+1)+2} \otimes w_{4(j+s)+1 \rightarrow 4(j+s+1)+1}, \quad i=(j+s+1)_{2 s}+2 s, j \geqslant 2 s ; \\
w_{4(j+m)+3 \rightarrow 4(j+m+s+1)+2} \otimes w_{4(j+s)+1 \rightarrow 4(j+s)+2}, \quad i=j+3 s ; \\
w_{4(j+m+1) \rightarrow 4(j+m+s+1)+2} \otimes w_{4(j+s)+1 \rightarrow 4 j+3}, \quad i=(j)_{s}+6 s ; \\
0 \quad \text { otherwise. }
\end{array}\right.
$$

If $3 s \leqslant j<5 s$, then

$$
\left(d_{1}\right)_{i j}=\left\{\begin{array}{l}
e_{4(j+m+1)+1} \otimes w_{4(j+s)+2 \rightarrow 4(j+1)}, \quad i=(j+1)_{2 s}, \quad j<4 s \\
e_{4(j+m+1)+1} \otimes w_{4(j+s)+2 \rightarrow 4(j+1)}, \quad i=j-4 s+1, j \geqslant 4 s \\
w_{4(j+m)+3 \rightarrow 4(j+m+1)+1} \otimes e_{4(j+s)+2}, \quad i=j+s \\
w_{4(j+m+1) \rightarrow 4(j+m+1)+1} \otimes w_{4(j+s)+2 \rightarrow 4 j+3}, \quad i=(j)_{s}+6 s \\
0 \quad \text { otherwise }
\end{array}\right.
$$


If $5 s \leqslant j<6 s$, then

$\left(d_{1}\right)_{i j}=\left\{\begin{array}{l}w_{4\left(j+m+\left(1-f\left(i_{2}, s-1\right)\right) s+1\right)+1 \rightarrow 4(j+m+2)} \otimes w_{4 j+3 \rightarrow 4(j+1)}, \quad i=(j+1)_{s} ; \\ w_{4\left(j+m+\left(1-f\left(i_{2}, s-1\right)\right) s+1\right)+2 \rightarrow 4(j+m+2)} \otimes w_{4 j+3 \rightarrow 4\left(j+\left(1-f\left(i_{2}, s-1\right)\right) s+1\right)+1}, \quad i=(j+1)_{s}+2 s \\ w_{4(j+m+1)+3 \rightarrow 4(j+m+2)} \otimes w_{4 j+3 \rightarrow 4\left(j+\left(1-f\left(i_{2}, s-1\right)\right) s+1\right)+2}, \quad i=(j+1)_{s}+4 s \\ w_{4(j+m+1) \rightarrow 4(j+m+2)} \otimes e_{4 j+3}, \quad i=j+s \\ e_{4(j+m+2)} \otimes w_{4 j+3 \rightarrow 4(j+1)+3}, \quad i=(j+1)_{s}+6 s \\ 0 \quad \text { otherwise. }\end{array}\right.$

Description of the $d_{2}$

If $0 \leqslant j<2 s$, then

$$
d_{2}: Q_{3} \rightarrow Q_{2}-\text { is an }(8 s \times 6 s) \text { matrix. }
$$

$$
\left(d_{2}\right)_{i j}=\left\{\begin{array}{l}
w_{4(j+m-1)+3 \rightarrow 4(j+m)+2} \otimes e_{4 j}, \quad i=(j)_{s} \\
-f_{1}(j, s) e_{4(j+m)+2} \otimes w_{4 j \rightarrow 4 j+1}, \quad i=j+s \\
f_{1}(j, s) w_{4(j+m)+1 \rightarrow 4(j+m)+2} \otimes w_{4 j \rightarrow 4(j+s)+2}, \quad i=(j+s)_{2 s}+3 s \\
0 \quad \text { otherwise. }
\end{array}\right.
$$

If $2 s \leqslant j<4 s$, then

$$
\left(d_{2}\right)_{i j}=\left\{\begin{array}{l}
-f_{1}(j, 3 s) f_{1}\left(i_{2}, s-1\right) e_{4(j+m)+3} \otimes w_{4 j+1 \rightarrow 4(j+1)}, \quad i=(j+1)_{s} ; \\
w_{4(j+m)+2 \rightarrow 4(j+m)+3} \otimes e_{4 j+1}, \quad i=j-s ; \\
-w_{4(j+m+s)+1 \rightarrow 4(j+m)+3} \otimes w_{4 j+1 \rightarrow 4 j+2}, \quad i=j+s \\
0 \quad \text { otherwise. }
\end{array}\right.
$$

If $4 s \leqslant j<6 s$, then

$$
\left(d_{2}\right)_{i j}=\left\{\begin{array}{l}
w_{4(j+m)+3 \rightarrow 4(j+m+1)} \otimes w_{4 j+2 \rightarrow 4(j+1)}, \quad i=(j+1)_{s}, j<5 s-1 \text { or } j=6 s-1 ; \\
w_{4(j+m+s)+1 \rightarrow 4(j+m+1)} \otimes e_{4 j+2}, \quad i=j-s \\
-e_{4(j+m+1)} \otimes w_{4 j+2 \rightarrow 4 j+3}, \quad i=(j)_{s}+5 s \\
0 \quad \text { otherwise. }
\end{array}\right.
$$

If $6 s \leqslant j<8 s$, then

$$
\left(d_{2}\right)_{i j}=\left\{\begin{array}{l}
-w_{4(j+m)+3 \rightarrow 4(j+m+1)+1} \otimes w_{4 j+3 \rightarrow 4(j+1)}, \quad i=(j+1)_{s}, j<7 s-1 \text { or } j=8 s-1 \\
-e_{4(j+m+1)+1} \otimes w_{4 j+3 \rightarrow 4(j+s+1)+2}, \quad i=(j+s+1)_{2 s}+3 s \\
w_{4(j+m+1) \rightarrow 4(j+m+1)+1} \otimes e_{4 j+3}, \quad i=(j)_{s}+5 s \\
0 \quad \text { otherwise. }
\end{array}\right.
$$


Description of the $d_{3}$

If $0 \leqslant j<2 s$, then

$$
d_{3}: Q_{4} \rightarrow Q_{3}-\text { is an }(9 s \times 8 s) \text { matrix. }
$$

$$
\left(d_{3}\right)_{i j}=\left\{\begin{array}{l}
w_{4\left(j+m+j_{2} s\right)+2 \rightarrow 4(j+m)+3+j_{2}} \otimes e_{4 j}, \quad i=(j)_{s} \\
-w_{4(j+m+s)+2+2 j_{2} \rightarrow 4(j+m)+3+j_{2}} \otimes w_{4 j \rightarrow 4 j+2 j_{2}}, \quad i=j+s+3 j_{2} s \\
e_{4(j+m)+3+j_{2}} \otimes w_{4 j \rightarrow 4\left(j+j_{2} s\right)+1+j_{2}}, \quad i=j+\left(2+j_{2}\right) s \\
w_{4(j+m)+3 \rightarrow 4(j+m)+3+j_{2}} \otimes w_{4 j \rightarrow 4(j+s)+1}, \quad i=(j+s)_{2 s}+2 s \\
0 \quad \text { otherwise. }
\end{array}\right.
$$

If $2 s \leqslant j<4 s$, then

$$
\left(d_{3}\right)_{i j}=\left\{\begin{array}{l}
w_{4(j+m)+3 \rightarrow 4(j+m+s+1)+1} \otimes e_{4 j+1}, \quad i=j \\
w_{4(j+m+1) \rightarrow 4(j+m+s+1)+1} \otimes w_{4 j+1 \rightarrow 4 j+2}, \quad i=j+2 s \\
e_{4(j+m+s+1)+1} \otimes w_{4 j+1 \rightarrow 4 j+3}, \quad i=(j+s)_{2 s}+6 s \\
0 \quad \text { otherwise. }
\end{array}\right.
$$

If $4 s \leqslant j<6 s$, then

$$
\left(d_{3}\right)_{i j}=\left\{\begin{array}{l}
-f_{1}(j, 6 s-1) e_{4(j+m+1)+2} \otimes w_{4(j+s)+2 \rightarrow 4(j+1)}, \quad i=(j+1)_{2 s}, \quad j \geqslant 5 s \\
w_{4(j+m+1) \rightarrow 4(j+m+1)+j_{2}-3} \otimes e_{4\left(j+\left(j_{2}-4\right) s\right)+2}, \quad i=(j)_{s}+4 s \\
w_{4(j+m+1)+1 \rightarrow 4(j+m+1)+j_{2}-3} \otimes w_{4\left(j+\left(j_{2}-4\right) s\right)+2 \rightarrow 4 j+3}, \quad i=j+2 s \\
0 \quad \text { otherwise. }
\end{array}\right.
$$

If $6 s \leqslant j<8 s$, then

$$
\left(d_{3}\right)_{i j}=\left\{\begin{array}{l}
f_{1}(j, 8 s-1) e_{4(j+m+s+1)+2} \otimes w_{4 j+2 \rightarrow 4(j+1)}, \quad i=j-7 s+1, j \geqslant 7 s \\
w_{4(j+m+1) \rightarrow 4(j+m+s+1)+1+j_{2}-6} \otimes e_{4\left(j+\left(7-j_{2}\right) s\right)+2}, \quad i=(j)_{s}+5 s \\
w_{4(j+m+s+1)+1 \rightarrow 4(j+m+s+1)+1+j_{2}-6} \otimes w_{4\left(j+\left(7-j_{2}\right) s\right)+2 \rightarrow 4 j+3}, \quad i=(j+s)_{2 s}+6 s \\
0 \quad \text { otherwise. }
\end{array}\right.
$$

If $8 s \leqslant j<9 s$, then

$$
\left(d_{3}\right)_{i j}=\left\{\begin{array}{l}
f_{1}(j, 9 s-1) w_{4(j+m+f(j, 9 s-1) s+1)+2 \rightarrow 4(j+m+1)+3} \otimes w_{4 j+3 \rightarrow 4(j+1)}, \quad i=(j+1)_{s} \\
f_{1}(j, 9 s-1) e_{4(j+m+1)+3} \otimes w_{4 j+3 \rightarrow 4(j+f(j, 9 s-1) s+1)+1}, \quad i=(j+1)_{s}+2 s \\
w_{4(j+m+1)+1 \rightarrow 4(j+m+1)+3} \otimes e_{4 j+3}, \quad i=j-2 s \\
-w_{4(j+m+s+1)+1 \rightarrow 4(j+m+1)+3} \otimes e_{4 j+3}, \quad i=j-s \\
0 \quad \text { otherwise. }
\end{array}\right.
$$




\section{Description of the $d_{4}$}

If $0 \leqslant j<2 s$, then

$$
d_{4}: Q_{5} \rightarrow Q_{4}-\text { is an }(8 s \times 9 s) \text { matrix. }
$$

$$
\left(d_{4}\right)_{i j}=\left\{\begin{array}{l}
w_{4(j+m-1)+3 \rightarrow 4(j+m)+1} \otimes e_{4 j}, \quad i=j, j<s \\
-f_{1}(j, s) w_{4(j+m) \rightarrow 4(j+m)+1} \otimes e_{4 j}, \quad i=(j)_{s}+s \\
-e_{4(j+m)+1} \otimes w_{4 j \rightarrow 4(j+s)+1}, \quad i=(j+s)_{2 s}+2 s \\
e_{4(j+m)+1} \otimes w_{4 j \rightarrow 4 j+2}, \quad i=j+\left(5-f_{0}(j, s)\right) s \\
0 \quad \text { otherwise. }
\end{array}\right.
$$

If $2 s \leqslant j<4 s$, then

$$
\left(d_{4}\right)_{i j}=\left\{\begin{array}{l}
w_{4(j+m)+3 \rightarrow 4(j+m+1)} \otimes w_{4 j+1 \rightarrow 4(j+1)}, \quad i=(j+1)_{s}, \quad j<3 s-1 \text { or } j=4 s-1 ; \\
-f_{1}\left(i_{2}, s-1\right) f_{1}(j, 3 s) e_{4(j+m+1)} \otimes w_{4 j+1 \rightarrow 4(j+1)}, \quad i=(j+1)_{s}+s ; \\
w_{4(j+m+s)+1 \rightarrow 4(j+m+1)} \otimes e_{4 j+1}, \quad i=j ; \\
-w_{4(j+m+s)+2 \rightarrow 4(j+m+1)} \otimes w_{4 j+1 \rightarrow 4 j+2}, \quad i=j+\left(4-f_{0}(j, 3 s)\right) s ; \\
0 \quad \text { otherwise. }
\end{array}\right.
$$

If $4 s \leqslant j<6 s$, then

$$
\left(d_{4}\right)_{i j}=\left\{\begin{array}{l}
e_{4(j+m)+3} \otimes w_{4 j+2 \rightarrow 4(j+1)}, \quad i=(j+1)_{s}, \quad j<5 s-1 \text { or } j=6 s-1 \\
w_{4(j+m)+1 \rightarrow 4(j+m)+3} \otimes e_{4 j+2}, \quad i=j+\left(1-f_{0}(j, 5 s)\right) s \\
-w_{4(j+m+s)+2 \rightarrow 4(j+m)+3} \otimes e_{4 j+2}, \quad i=j+\left(2-f_{0}(j, 5 s)\right) s \\
-f_{1}(j, 5 s) e_{4(j+m)+3} \otimes w_{4 j+2 \rightarrow 4 j+3}, \quad i=(j)_{s}+8 s \\
0 \quad \text { otherwise. }
\end{array}\right.
$$

If $6 s \leqslant j<8 s$, then

$$
\left(d_{4}\right)_{i j}=\left\{\begin{array}{l}
-f_{1}(j, 7 s) w_{4(j+m)+3 \rightarrow 4(j+m+1)+2} \otimes w_{4 j+3 \rightarrow 4(j+1)} \\
i=(j+1)_{s}, j<7 s-1 \text { or } j=8 s-1 ; \\
f_{1}(j, 7 s) w_{4(j+m+1)+1 \rightarrow 4(j+m+1)+2} \otimes w_{4 j+3 \rightarrow 4(j+s+1)+1}, \quad i=(j+s+1)_{2 s}+2 s \\
-f_{1}(j, 7 s) e_{4(j+m+1)+2} \otimes w_{4 j+3 \rightarrow 4(j+s+1)+2}, \\
i=(j+1)_{s}+7 s, j<7 s-1 \text { or } j=8 s-1 ; \\
-f_{1}(j, 7 s) e_{4(j+m+1)+2} \otimes w_{4 j+3 \rightarrow 4(j+s+1)+2}, \quad i=(j+1)_{s}+5 s, 7 s-1 \leqslant j<8 s-1 \\
w_{4(j+m)+3 \rightarrow 4(j+m+1)+2} \otimes e_{4 j+3}, \quad i=(j)_{s}+8 s \\
0 \quad \text { otherwise. }
\end{array}\right.
$$


Description of the $d_{5}$

If $0 \leqslant j<s$, then

$$
d_{5}: Q_{6} \rightarrow Q_{5}-\text { is an }(9 s \times 8 s) \text { matrix. }
$$

$$
\left(d_{5}\right)_{i j}=\left\{\begin{array}{l}
f_{1}\left(j_{1}, 2\right) w_{4(j+m)+1+j_{1}+2 f\left(j_{1}, 1\right) \rightarrow 4(j+m+1)} \otimes w_{4 j \rightarrow 4 j+j_{1}}, \quad i=j+2 j_{1} s, 0 \leqslant j_{1} \leqslant 2 \\
f_{1}\left(j_{1}, 2\right) w_{4(j+m+s)+1+j_{1}+2 f\left(j_{1}, 1\right) \rightarrow 4(j+m+1)} \otimes w_{4 j \rightarrow 4(j+s)+j_{1}}, \\
\quad i=j+\left(2 j_{1}+1\right) s, 0 \leqslant j_{1} \leqslant 2 \\
\quad \text { otherwise. }
\end{array}\right.
$$

If $s \leqslant j<3 s$, then

$$
\left(d_{5}\right)_{i j}=\left\{\begin{array}{l}
-f_{1}(j, 2 s) w_{4(j+m+s+1)+1 \rightarrow 4(j+m+s+1)+j_{2}} \otimes w_{4\left(j+s\left(2-j_{2}\right)\right)+1 \rightarrow 4(j+1)}, \quad i=(j+s+1)_{2 s} ; \\
w_{4(j+m+1) \rightarrow 4(j+m+s+1)+j_{2}} \otimes e_{4\left(j+s\left(2-j_{2}\right)\right)+1}, \quad i=(j)_{s}+2 s ; \\
-w_{4(j+m)+3 \rightarrow 4(j+m+s+1)+2} \otimes w_{4 j+1 \rightarrow 4 j+2}, \quad i=j+2 s, j \geqslant 2 s ; \\
-e_{4(j+m+s+1)+2} \otimes w_{4 j+1 \rightarrow 4 j+3}, \quad i=j+5 s, j \geqslant 2 s ; \\
0 \quad \text { otherwise. }
\end{array}\right.
$$

If $3 s \leqslant j<5 s$, then

$$
\left(d_{5}\right)_{i j}=\left\{\begin{array}{l}
-f_{1}(j, 4 s) w_{4(j+m+1)+1 \rightarrow 4(j+m+1)+j_{2}-2} \otimes w_{4\left(j+s\left(j_{2}-3\right)\right)+1 \rightarrow 4(j+1)}, \quad i=(j+1)_{2 s} \\
w_{4(j+m+1) \rightarrow 4(j+m+1)+j_{2}-2} \otimes e_{4\left(j+s\left(j_{2}-3\right)\right)+1}, \quad i=(j)_{s}+3 s ; \\
-w_{4(j+m)+3 \rightarrow 4(j+m+1)+2} \otimes w_{4(j+s)+1 \rightarrow 4(j+s)+2}, \quad i=j+s, j \geqslant 4 s \\
e_{4(j+m+1)+2} \otimes w_{4(j+s)+1 \rightarrow 4 j+3}, \quad i=j+2 s, j \geqslant 4 s ; \\
0 \quad \text { otherwise. }
\end{array}\right.
$$

If $5 s \leqslant j<7 s$, then

$$
\left(d_{5}\right)_{i j}=\left\{\begin{array}{l}
w_{4(j+m)+3 \rightarrow 4(j+m+s+1)+2} \otimes e_{4(j+s)+2}, \quad i=j-s \\
f_{1}(j, 6 s) e_{4(j+m+s+1)+2} \otimes w_{4(j+s)+2 \rightarrow 4 j+3}, \quad i=j+s \\
0 \quad \text { otherwise. }
\end{array}\right.
$$

If $7 s \leqslant j<8 s$, then

$$
\left(d_{5}\right)_{i j}=\left\{\begin{array}{l}
w_{4(j+m+s+1)+1 \rightarrow 4(j+m+1)+3} \otimes w_{4 j+3 \rightarrow 4(j+1)}, \quad i=j-7 s+1 ; \\
w_{4(j+m+1)+1 \rightarrow 4(j+m+1)+3} \otimes w_{4 j+3 \rightarrow 4(j+1)}, \quad i=(j+1)_{2 s} ; \\
-e_{4(j+m+1)+3} \otimes w_{4 j+3 \rightarrow 4(j+s+1)+2}, \quad i=j-3 s+1 ; \\
-e_{4(j+m+1)+3} \otimes w_{4 j+3 \rightarrow 4(j+1)+2}, \quad i=(j+1)_{2 s}+4 s ; \\
w_{4(j+m+s+1)+2 \rightarrow 4(j+m+1)+3} \otimes e_{4 j+3}, \quad i=j-s ; \\
-w_{4(j+m+1)+2 \rightarrow 4(j+m+1)+3} \otimes e_{4 j+3}, \quad i=j ; \\
0 \quad \text { otherwise. }
\end{array}\right.
$$


If $8 s \leqslant j<9 s$, then

$$
\left(d_{5}\right)_{i j}=\left\{\begin{array}{l}
-e_{4(j+m+2)} \otimes w_{4 j+3 \rightarrow 4(j+s+1)+1}, \quad i=(j+s+1)_{2 s}+2 s \\
w_{4(j+m+1)+2 \rightarrow 4(j+m+2)} \otimes e_{4 j+3}, \quad i=j-2 s \\
0 \quad \text { otherwise. }
\end{array}\right.
$$

Description of the $d_{6}$

If $0 \leqslant j<2 s$, then

$$
d_{6}: Q_{7} \rightarrow Q_{6}-\text { is an }(8 s \times 9 s) \text { matrix. }
$$

$$
\left(d_{6}\right)_{i j}=\left\{\begin{array}{l}
w_{4(j+m) \rightarrow 4(j+m)+2} \otimes e_{4 j}, \quad i=(j)_{s} ; \\
-w_{4(j+m)+1 \rightarrow 4(j+m)+2} \otimes w_{4 j \rightarrow 4 j+1}, \quad i=j+\left(j_{2}+1\right) s \\
-e_{4(j+m)+2} \otimes w_{4 j \rightarrow 4(j+s)+1}, \quad i=j+\left(4-3 j_{2}\right) s \\
e_{4(j+m)+2} \otimes w_{4 j \rightarrow 4 j+2}, \quad i=j+5 s \\
0 \quad \text { otherwise. }
\end{array}\right.
$$

If $2 s \leqslant j<4 s$, then

$$
\left(d_{6}\right)_{i j}=\left\{\begin{array}{l}
w_{4(j+m)+1 \rightarrow 4(j+m)+3} \otimes e_{4 j+1}, \quad i=j+s\left(j_{2}-3\right) \\
-w_{4(j+m+s)+2 \rightarrow 4(j+m)+3} \otimes e_{4 j+1}, \quad i=j+s\left(j_{2}-2\right) \\
-w_{4(j+m)+2 \rightarrow 4(j+m)+3} \otimes w_{4 j+1 \rightarrow 4 j+2}, \quad i=j+3 s \\
e_{4(j+m)+3} \otimes w_{4 j+1 \rightarrow 4 j+3}, \quad i=(j)_{s}+7 s \\
0 \quad \text { otherwise. }
\end{array}\right.
$$

If $4 s \leqslant j<6 s$, then

$$
\left(d_{6}\right)_{i j}=\left\{\begin{array}{l}
e_{4(j+m+1)} \otimes w_{4 j+2 \rightarrow 4(j+1)}, \quad i=(j+1)_{s}, j \geqslant 5 s \\
w_{4(j+m)+2 \rightarrow 4(j+m+1)} \otimes e_{4 j+2}, \quad i=j+s ; \\
-w_{4(j+m)+3 \rightarrow 4(j+m+1)} \otimes w_{4 j+2 \rightarrow 4 j+3}, \quad i=j+2 s, j \geqslant 5 s \\
-f_{1}(j, 5 s) e_{4(j+m+1)} \otimes w_{4 j+2 \rightarrow 4 j+3}, \quad i=(j)_{s}+8 s ; \\
0 \quad \text { otherwise. }
\end{array}\right.
$$

If $6 s \leqslant j<8 s$, then

$$
\left(d_{6}\right)_{i j}=\left\{\begin{array}{l}
-w_{4(j+m+1) \rightarrow 4(j+m+1)+1} \otimes w_{4 j+3 \rightarrow 4(j+1)}, \quad i=(j+1)_{s}, j<7 s ; \\
e_{4(j+m+1)+1} \otimes w_{4 j+3 \rightarrow 4(j+1)+1}, \quad i=(j+1)_{2 s}+s, j<7 s-1 \text { or } j=8 s-1 ; \\
e_{4(j+m+1)+1} \otimes w_{4 j+3 \rightarrow 4(j+1)+1}, \quad i=(j+1)_{2 s}+2 s, 7 s-1 \leqslant j<8 s-1 ; \\
w_{4(j+m)+3 \rightarrow 4(j+m+1)+1} \otimes e_{4 j+3}, \quad i=j+s, j<7 s ; \\
-f_{1}(j, 7 s) w_{4(j+m+1) \rightarrow 4(j+m+1)+1} \otimes e_{4 j+3}, \quad i=(j)_{s}+8 s ; \\
0 \quad \text { otherwise. }
\end{array}\right.
$$


If $0 \leqslant j<s$, then

Description of the $d_{7}$

$$
d_{7}: Q_{8} \rightarrow Q_{7}-\text { is an }(6 s \times 8 s) \text { matrix. }
$$

$$
\left(d_{7}\right)_{i j}=\left\{\begin{array}{l}
w_{4(j+m)+2 \rightarrow 4(j+m)+3} \otimes e_{4 j}, \quad i=j \\
-w_{4(j+m+s)+2 \rightarrow 4(j+m)+3} \otimes e_{4 j}, \quad i=j+s \\
e_{4(j+m)+3} \otimes w_{4 j \rightarrow 4 j+1}, \quad i=j+2 s \\
-e_{4(j+m)+3} \otimes w_{4 j \rightarrow 4(j+s)+1}, \quad i=j+3 s \\
0 \quad \text { otherwise. }
\end{array}\right.
$$

If $s \leqslant j<3 s$, then

$$
\left(d_{7}\right)_{i j}=\left\{\begin{array}{l}
-e_{4(j+m+s+1)+2} \otimes w_{4(j+s)+1 \rightarrow 4(j+1)}, \quad i=(j+s+1)_{2 s} \\
w_{4(j+m)+3 \rightarrow 4(j+m+s+1)+2} \otimes e_{4(j+s)+1}, \quad i=j+s \\
w_{4(j+m+1) \rightarrow 4(j+m+s+1)+2} \otimes w_{4(j+s)+1 \rightarrow 4(j+s)+2}, \quad i=j+3 s \\
-w_{4(j+m+s+1)+1 \rightarrow 4(j+m+s+1)+2} \otimes w_{4(j+s)+1 \rightarrow 4 j+3}, \quad i=j+5 s \\
0 \quad \text { otherwise. }
\end{array}\right.
$$

If $3 s \leqslant j<5 s$, then

$$
\left(d_{7}\right)_{i j}=\left\{\begin{array}{l}
w_{4(j+m+1) \rightarrow 4(j+m+1)+1} \otimes e_{4(j+s)+2}, \quad i=j+s \\
e_{4(j+m+1)+1} \otimes w_{4(j+s)+2 \rightarrow 4 j+3}, \quad i=(j)_{2 s}+6 s \\
0 \quad \text { otherwise. }
\end{array}\right.
$$

If $5 s \leqslant j<6 s$, then

$$
\left(d_{7}\right)_{i j}=\left\{\begin{array}{l}
w_{4(j+m+1+s f(j, 6 s-1))+2 \rightarrow 4(j+m+2)} \otimes w_{4 j+3 \rightarrow 4(j+1)}, \quad i=(j+1)_{s}+s ; \\
-w_{4(j+m+1)+3 \rightarrow 4(j+m+2)} \otimes w_{4 j+3 \rightarrow 4(j+1+(1+f(j, 6 s-1)) s)+1}, \quad i=(j+1)_{s}+2 s ; \\
-e_{4(j+m+2)} \otimes w_{4 j+3 \rightarrow 4(j+1+(1+f(j, 6 s-1)) s)+2}, \quad i=(j+1)_{s}+4 s ; \\
-e_{4(j+m+2)} \otimes w_{4 j+3 \rightarrow 4(j+1+s f(j, 6 s-1))+2}, \quad i=(j+1)_{s}+5 s ; \\
w_{4(j+m+s+1)+1 \rightarrow 4(j+m+2)} \otimes e_{4 j+3}, \quad i=j+s ; \\
w_{4(j+m+1)+1 \rightarrow 4(j+m+2)} \otimes e_{4 j+3}, \quad i=j+2 s ; \\
0 \quad \text { otherwise. }
\end{array}\right.
$$




\section{Description of the $d_{8}$}

If $0 \leqslant j<s$, then

$$
d_{8}: Q_{9} \rightarrow Q_{8}-\text { is an }(7 s \times 6 s) \text { matrix. }
$$

$$
\left(d_{8}\right)_{i j}=\left\{\begin{array}{l}
w_{4(j+m-1)+3 \rightarrow 4(j+m)+3} \otimes e_{4 j}, \quad i=j ; \\
-f_{1}(j, s-1) e_{4(j+m)+3} \otimes w_{4 j \rightarrow 4(j+1)}, \quad i=(j+1)_{s} \\
-w_{4(j+m)+2 \rightarrow 4(j+m)+3} \otimes w_{4 j \rightarrow 4 j+1}, \quad i=j+s \\
w_{4(j+m+s)+2 \rightarrow 4(j+m)+3} \otimes w_{4 j \rightarrow 4(j+s)+1}, \quad i=j+2 s \\
w_{4(j+m+s)+1 \rightarrow 4(j+m)+3} \otimes w_{4 j \rightarrow 4 j+2}, \quad i=j+3 s \\
-w_{4(j+m)+1 \rightarrow 4(j+m)+3} \otimes w_{4 j \rightarrow 4(j+s)+2}, \quad i=j+4 s \\
0 \quad \text { otherwise. }
\end{array}\right.
$$

If $s \leqslant j<3 s$, then

$$
\left(d_{8}\right)_{i j}=\left\{\begin{array}{l}
w_{4(j+m)+3 \rightarrow 4(j+m+1)} \otimes w_{4(j+s)+1 \rightarrow 4(j+1)}, \quad i=(j+1)_{s}, j<2 s-1 \text { or } j=3 s-1 ; \\
w_{4(j+m+s)+2 \rightarrow 4(j+m+1)} \otimes e_{4(j+s)+1}, \quad i=j ; \\
-w_{4(j+m)+1 \rightarrow 4(j+m+1)} \otimes w_{4(j+s)+1 \rightarrow 4(j+s)+2}, \quad i=j+2 s \\
e_{4(j+m+1)} \otimes w_{4(j+s)+1 \rightarrow 4 j+3}, \quad i=(j)_{s}+5 s ; \\
0 \quad \text { otherwise. }
\end{array}\right.
$$

If $3 s \leqslant j<5 s$, then

$$
\left(d_{8}\right)_{i j}=\left\{\begin{array}{l}
-w_{4(j+m)+3 \rightarrow 4(j+m+1)+1} \otimes w_{4(j+s)+2 \rightarrow 4(j+1)}, \quad i=(j+1)_{s}, j<4 s-1 \text { or } j=5 s-1 ; \\
w_{4(j+m)+1 \rightarrow 4(j+m+1)+1} \otimes e_{4(j+s)+2}, \quad i=j ; \\
-e_{4(j+m+1)+1} \otimes w_{4(j+s)+2 \rightarrow 4(j+s+1)+2}, \quad i=(j+s+1)_{2 s}+3 s ; \\
-w_{4(j+m+1) \rightarrow 4(j+m+1)+1} \otimes w_{4(j+s)+2 \rightarrow 4 j+3}, \quad i=(j)_{s}+5 s \\
0 \quad \text { otherwise. }
\end{array}\right.
$$

If $5 s \leqslant j<7 s$, then

$$
\left(d_{8}\right)_{i j}=\left\{\begin{array}{l}
w_{4(j+m)+3 \rightarrow 4(j+m+s+1)+2} \otimes w_{4 j+3 \rightarrow 4(j+1)}, \quad i=(j+1)_{s}, 6 s-1 \leqslant j<7 s-1 \\
e_{4(j+m+s+1)+2} \otimes w_{4 j+3 \rightarrow 4(j+s+1)+1}, \quad i=(j+s+1)_{2 s}+s \\
w_{4(j+m+s+1)+1 \rightarrow 4(j+m+s+1)+2} \otimes w_{4 j+3 \rightarrow 4(j+1)+2}, \quad i=(j+1)_{2 s}+3 s \\
w_{4(j+m+1) \rightarrow 4(j+m+s+1)+2} \otimes e_{4 j+3}, \quad i=(j)_{s}+5 s \\
0 \quad \text { otherwise. }
\end{array}\right.
$$


If $0 \leqslant j<s$, then

Description of the $d_{9}$

$d_{9}: Q_{10} \rightarrow Q_{9}-$ is an $(6 s \times 7 s)$ matrix.

$$
\left(d_{9}\right)_{i j}=\left\{\begin{array}{l}
w_{4(j+m)+3 \rightarrow 4(j+m+1)} \otimes e_{4 j}, \quad i=j \\
e_{4(j+m+1)} \otimes w_{4 j \rightarrow 4 j+1}, \quad i=j+s \\
-e_{4(j+m+1)} \otimes w_{4 j \rightarrow 4(j+s)+1}, \quad i=j+2 s \\
0 \quad \text { otherwise. }
\end{array}\right.
$$

If $s \leqslant j<3 s$, then

$$
\left(d_{9}\right)_{i j}= \begin{cases}w_{4(j+m+1) \rightarrow 4(j+m+1)+1} \otimes e_{4(j+s)+1}, & i=j \\ e_{4(j+m+1)+1} \otimes w_{4(j+s)+1 \rightarrow 4(j+s)+2}, & i=j+2 s \\ 0 \quad \text { otherwise } & \end{cases}
$$

If $3 s \leqslant j<5 s$, then

$$
\left(d_{9}\right)_{i j}=\left\{\begin{array}{l}
w_{4(j+m+1)+1 \rightarrow 4(j+m+1)+2} \otimes e_{4(j+s)+2}, \quad i=j \\
e_{4(j+m+1)+2} \otimes w_{4(j+s)+2 \rightarrow 4 j+3}, \quad i=(j)_{2 s}+5 s \\
0 \quad \text { otherwise }
\end{array}\right.
$$

If $5 s \leqslant j<6 s$, then

$$
\left(d_{9}\right)_{i j}=\left\{\begin{array}{l}
f_{1}(j, 6 s-1) e_{4(j+m+1)+3} \otimes w_{4 j+3 \rightarrow 4(j+1)}, \quad i=(j+1)_{s} \\
w_{4(j+m+s+1)+2 \rightarrow 4(j+m+1)+3} \otimes e_{4 j+3}, \quad i=j \\
-w_{4(j+m+1)+2 \rightarrow 4(j+m+1)+3} \otimes e_{4 j+3}, \quad i=j+s \\
0 \quad \text { otherwise. }
\end{array}\right.
$$

If $0 \leqslant j<s$, then

\section{Description of the $d_{10}$}

$$
d_{10}: Q_{11} \rightarrow Q_{10}-\text { is an }(6 s \times 6 s) \text { matrix. }
$$

$$
\left(d_{10}\right)_{i j}=\left\{\begin{array}{l}
-f_{1}(j, s-1) e_{4(j+m+1)} \otimes w_{4 j \rightarrow 4(j+1)}, \quad i=(j+1)_{s} ; \\
-f_{1}\left(j_{1}, 1\right) w_{4(j+m+s)+1+j_{1} \rightarrow 4(j+m+1)} \otimes w_{4 j \rightarrow 4 j+1+j_{1}}, \quad i=j+\left(2 j_{1}+1\right) s, \quad 0 \leqslant j_{1} \leqslant 2 \\
f_{1}\left(j_{1}, 2\right) w_{4(j+m)+j_{1} \rightarrow 4(j+m+1)} \otimes w_{4 j \rightarrow 4(j+s)+j_{1}}, \quad i=j+2 j_{1} s, \quad 0 \leqslant j_{1} \leqslant 2 \\
0 \quad \text { otherwise. }
\end{array}\right.
$$


If $s \leqslant j<3 s$, then

$$
\left(d_{10}\right)_{i j}=\left\{\begin{array}{l}
f_{1}\left(i_{2}, s-1\right) f_{1}(j, 2 s) w_{4(j+m+1) \rightarrow 4(j+m+1)+1} \otimes w_{4(j+s)+1 \rightarrow 4(j+1)}, \quad i=(j+1)_{s} ; \\
w_{4(j+m)+1 \rightarrow 4(j+m+1)+1} \otimes e_{4(j+s)+1}, \quad i=j ; \\
-e_{4(j+m+1)+1} \otimes w_{4(j+s)+1 \rightarrow 4(j+s+1)+1}, \quad i=(j+s+1)_{2 s}+s ; \\
-w_{4(j+m)+2 \rightarrow 4(j+m+1)+1} \otimes w_{4(j+s)+1 \rightarrow 4(j+s)+2}, \quad i=j+2 s ; \\
-f_{1}(j, 2 s) w_{4(j+m)+3 \rightarrow 4(j+m+1)+1} \otimes w_{4(j+s)+1 \rightarrow 4 j+3}, \quad i=(j)_{s}+5 s \\
0 \quad \text { otherwise. }
\end{array}\right.
$$

If $3 s \leqslant j<5 s$, then

$$
\left(d_{10}\right)_{i j}=\left\{\begin{array}{l}
-f_{1}\left(i_{2}, s-1\right) f_{1}(j, 4 s) w_{4(j+m+1) \rightarrow 4(j+m+1)+2} \otimes w_{4(j+s)+2 \rightarrow 4(j+1)}, \quad i=(j+1)_{s} \\
w_{4(j+m+1)+1 \rightarrow 4(j+m+1)+2} \otimes w_{4(j+s)+2 \rightarrow 4(j+s+1)+1}, \quad i=(j+s+1)_{2 s}+s \\
w_{4(j+m)+2 \rightarrow 4(j+m+1)+2} \otimes e_{4(j+s)+2}, \quad i=j ; \\
-e_{4(j+m+1)+2} \otimes w_{4(j+s)+2 \rightarrow 4(j+s+1)+2}, \quad i=(j+s+1)_{2 s}+3 s ; \\
f_{1}(j, 4 s) w_{4(j+m)+3 \rightarrow 4(j+m+1)+2} \otimes w_{4(j+s)+2 \rightarrow 4 j+3}, \quad i=(j)_{s}+5 s \\
0 \quad \text { otherwise. }
\end{array}\right.
$$

If $5 s \leqslant j<6 s$, then

$$
\left(d_{10}\right)_{i j}=\left\{\begin{array}{l}
-f_{1}(j, 6 s-1) w_{4(j+m+1) \rightarrow 4(j+m+1)+3} \otimes w_{4 j+3 \rightarrow 4(j+1)}, \quad i=(j+1)_{s} ; \\
w_{4(j+m+1)+1 \rightarrow 4(j+m+1)+3} \otimes w_{4 j+3 \rightarrow 4(j+s+1)+1}, \quad i=j-4 s+1 ; \\
-w_{4(j+m+s+1)+1 \rightarrow 4(j+m+1)+3} \otimes w_{4 j+3 \rightarrow 4(j+1)+1}, \quad i=(j+1)_{2 s}+s ; \\
-w_{4(j+m+1)+2 \rightarrow 4(j+m+1)+3} \otimes w_{4 j+3 \rightarrow 4(j+s+1)+2}, \quad i=j-2 s+1 ; \\
w_{4(j+m+s+1)+2 \rightarrow 4(j+m+1)+3} \otimes w_{4 j+3 \rightarrow 4(j+1)+2}, \quad i=(j+1)_{2 s}+3 s ; \\
w_{4(j+m)+3 \rightarrow 4(j+m+1)+3} \otimes e_{4 j+3}, \quad i=j ; \\
-f_{1}(j, 6 s-1) e_{4(j+m+1)+3} \otimes w_{4 j+3 \rightarrow 4(j+1)+3}, \quad i=(j+1)_{s}+5 s ; \\
0 \quad \text { otherwise. }
\end{array}\right.
$$

Theorem 3. Let $R=R_{s}^{\prime}$ is algebra of the type $E_{6}^{\prime}$. Then the minimal projective resolution of the $\Lambda$-module $R$ is of the form:

$$
\cdots \longrightarrow Q_{3} \stackrel{d_{2}}{\longrightarrow} Q_{2} \stackrel{d_{1}}{\longrightarrow} Q_{1} \stackrel{d_{0}}{\longrightarrow} Q_{0} \stackrel{\varepsilon}{\longrightarrow} R \longrightarrow 0,
$$

where $\varepsilon$ is the multiplication map $(\varepsilon(a \otimes b)=a b) ; Q_{r}(r \leqslant 10)$ and $d_{r}(r \leqslant 10)$ were described before; further $Q_{11 \ell+r}$, where $\ell \in \mathbb{N}$ and $0 \leqslant r \leqslant 10$, is obtained from $Q_{r}$ by replacing every direct summand $P_{i, j}$ to $P_{\sigma^{\ell}(i), j}$ correspondingly $\left(\right.$ here $\sigma(i)=j$, if $\left.\sigma\left(e_{i}\right)=e_{j}\right)$, and the differential $d_{11 \ell+r}$ is obtained from $d_{r}$ by act of $\sigma^{\ell}$ by all left tensor components of the corresponding matrix.

To prove that the terms $Q_{i}$ are of this form we introduce $P_{i}=R e_{i}$ is the projective cover of the simple $R$-modules $S_{i}$, corresponding to the vertices of the quiver $\mathcal{Q}_{s}$. We will find projective resolutions of the simple $R$-modules $S_{i}$. 
Denotation. For $R$-module $M$ its $m$ th syzygy is denoted by $\Omega^{m}(M)$.

Remark 5. From here we denote the multiplication homomorphism from the right by an element $w$ by $w$.

Lemma 4. The begin of the minimal projective resolution of $S_{4 r}$ is of the form

$$
\begin{aligned}
\cdots \longrightarrow P_{4(r+3)+3} & \stackrel{\left(\begin{array}{c}
\alpha \\
-\alpha
\end{array}\right)}{\longrightarrow} P_{4(r+3)+2} \oplus P_{4(r+s+3)+2} \stackrel{\left(\alpha^{2} \alpha^{2}\right)}{\longrightarrow} \\
\longrightarrow P_{4(r+3)} & \stackrel{\left(\begin{array}{c}
\gamma \alpha^{2} \\
\gamma \alpha^{2}
\end{array}\right)}{\longrightarrow} P_{4(r+2)+1} \oplus P_{4(r+s+2)+1} \stackrel{\left(\begin{array}{cc}
\alpha \gamma & 0 \\
-\alpha & \alpha
\end{array}\right)}{\longrightarrow} \\
\longrightarrow P_{4(r+1)+3} \oplus P_{4(r+2)} \stackrel{\left(\begin{array}{cc}
\alpha & \gamma \alpha \\
-\alpha & 0
\end{array}\right)}{\longrightarrow} P_{4(r+1)+2} \oplus P_{4(r+s+1)+2} \stackrel{\left(\alpha^{2} \gamma \alpha^{2} \gamma\right)}{\longrightarrow} & \\
\longrightarrow P_{4 r+3} & \stackrel{\left(\begin{array}{c}
\alpha^{2} \\
-\alpha^{2}
\end{array}\right)}{\longrightarrow} P_{4 r+1} \oplus P_{4(r+s)+1} \stackrel{(\alpha \alpha)}{\longrightarrow} P_{4 r} \longrightarrow S_{4 r} \longrightarrow 0 .
\end{aligned}
$$

At that $\Omega^{9}\left(S_{4 r}\right) \simeq S_{4(r+4)+3}$.

Lemma 5. The begin of the minimal projective resolution of $S_{4 r+1}$ is of the form

$$
\cdots \longrightarrow P_{4 r+2} \stackrel{\alpha}{\longrightarrow} P_{4 r+1} \longrightarrow S_{4 r+1} \longrightarrow 0 .
$$

At that $\Omega^{2}\left(S_{4 r+1}\right) \simeq S_{4(r+1)+2}$.

Lemma 6. The begin of the minimal projective resolution of $S_{4 r+2}$ is of the form

$$
\begin{aligned}
\cdots \longrightarrow P_{4(r+s+4)+1} \stackrel{\alpha}{\longrightarrow} P_{4(r+4)} \stackrel{\gamma \alpha}{\longrightarrow} P_{4(r+3)+2} \stackrel{\alpha^{2} \gamma}{\longrightarrow} P_{4(r+2)+3} \stackrel{\left(\alpha^{2}\right)}{\longrightarrow} \\
\longrightarrow P_{4(r+2)+1} \oplus P_{4(r+s+2)+2} \stackrel{\left(\alpha \alpha^{2}\right)}{\longrightarrow} P_{4(r+2)} \stackrel{\gamma \alpha^{2}}{\longrightarrow} \\
\longrightarrow P_{4(r+s+1)+1} \stackrel{\alpha \gamma}{\longrightarrow} P_{4 r+3} \stackrel{\alpha}{\longrightarrow} P_{4 r+2} \longrightarrow S_{4 r+2} \longrightarrow 0 .
\end{aligned}
$$

At that $\Omega^{9}\left(S_{4 r+2}\right) \simeq S_{4(r+s+5)+1}$.

Lemma 7. The begin of the minimal projective resolution of $S_{4 r+3}$ is of the form

$$
\cdots \longrightarrow P_{4(r+1)} \stackrel{\gamma}{\longrightarrow} P_{4 r+3} \longrightarrow S_{4 r+3} \longrightarrow 0 .
$$

At that $\Omega^{2}\left(S_{4 r+3}\right) \simeq S_{4(r+2)}$.

Proof. Proofs of the lemmas consist of direct check that given sequences are exact, and it is immediate.

We shall need the Happel's lemma (see [13]), as revised in [3]:

Lemma 8 (Happel). Let

$$
\cdots \rightarrow Q_{m} \rightarrow Q_{m-1} \rightarrow \cdots \rightarrow Q_{1} \rightarrow Q_{0} \rightarrow R \rightarrow 0
$$

be the minimal projective resolution of $R$. Then

$$
Q_{m} \cong \bigoplus_{i, j} P_{i, j}^{\operatorname{dim} \operatorname{Ext}_{R}^{m}\left(S_{j}, S_{i}\right)} .
$$


Proof of the theorem [3. Descriptions for $Q_{i}$ immediately follows from lemmas 4-7 and Happel's lemma.

As proved in [14], to prove that sequence (\#) is exact in $Q_{m}(m \leqslant 11)$ it will be sufficient to show that $d_{m} d_{m+1}=0$. It is easy to verify this relation by a straightforward calculation of matrixes products.

Since the sequence is exact in $Q_{11}$, it follows that $\left.\Omega^{11}{ }_{\Lambda} R\right) \simeq{ }_{1} R_{\sigma}$, where $\Omega^{11}\left({ }_{\Lambda} R\right)=\operatorname{Im} d_{10}$ is the 11th syzygy of the module $R$, and ${ }_{1} R_{\sigma}$ is a twisted bimodule. Hence, an exactness in $Q_{t}$ $(t>11)$ holds.

We recall that for $R$-bimodule $M$ the twisted bimodule is a linear space $M$, on which left act right acts of the algebra $R$ (denoted by asterisk) are assigned by the following way:

$$
r * m * s=\lambda(r) \cdot m \cdot \mu(s) \text { for } r, s \in R \text { and } m \in M,
$$

where $\lambda, \mu$ are some automorphisms of algebra $R$. Such twisted bimodule we shall denote by ${ }_{\lambda} M_{\mu}$.

Corollary 9. We have isomorphism $\Omega^{11}\left({ }_{\Lambda} R\right) \simeq{ }_{1} R_{\sigma}$.

Proposition 10. Automorphism $\sigma$ has a finite order, and

(1) if char $K=2$, then order of $\sigma$ is equal to $\frac{2 s}{\operatorname{gcd}(n+s, 2 s)}$;

(2) if char $K \neq 2$, then order of $\sigma$ is equal to $\frac{2 s}{\operatorname{gcd}(n+s, 2 s)}$, if $\frac{2 s}{\operatorname{gcd}(n+s, 2 s)}$ is divisible by 4 , and to $\frac{4 s}{\operatorname{gcd}(n+s, 2 s)}$ otherwise.

Proposition 11. The minimal period of bimodule resolution of $R$ is $11 \operatorname{deg} \sigma$.

\section{The Additive Structure of $\operatorname{HH}^{*}(R)$}

Proposition 12 (Dimensions of homomorphism groups, $s>1$ ). Let $s>1$ and $R=R_{s}^{\prime}$ is algebra of the type $E_{6}$. Next, $t \in \mathbb{N} \cup\{0\}$, $\ell$ be the aliquot, and $r$ be the residue of division of $t$ by 11 .

(1) If $r=0$, then

$$
\operatorname{dim}_{K} \operatorname{Hom}_{\Lambda}\left(Q_{d e g}, R\right)= \begin{cases}6 s, & \ell(n+s)+m \equiv 0(2 s) \text { or } \ell(n+s)+m \equiv 1(2 s) \\ 2 s, & \ell(n+s)+m \equiv s(2 s) \text { or } \ell(n+s)+m \equiv s+1(2 s) \\ 0, & \text { otherwise. }\end{cases}
$$

(2) If $r=1$, then

$$
\operatorname{dim}_{K} \operatorname{Hom}_{\Lambda}\left(Q_{\text {deg }}, R\right)= \begin{cases}7 s, & \ell(n+s)+m \equiv 0(2 s) \\ 5 s, & \ell(n+s)+m \equiv s(2 s) \\ 0, & \text { otherwise. }\end{cases}
$$

(3) If $r \in\{2,8\}$, then

$$
\operatorname{dim}_{K} \operatorname{Hom}_{\Lambda}\left(Q_{d e g}, R\right)= \begin{cases}3 s, & \ell(n+s)+m \equiv 0(2 s) \text { or } \ell(n+s)+m \equiv s+1(2 s) \\ s, & \ell(n+s)+m \equiv s(2 s) \text { or } \ell(n+s)+m \equiv 1(2 s) \\ 0, & \text { otherwise. }\end{cases}
$$


HOCHSCHILD COHOMOLOGY RING FOR SELF-INJECTIVE ALGEBRAS OF TREE CLASS E $E_{6}$ II. 21

(4) If $r \in\{3,5,7\}$, then

$$
\operatorname{dim}_{K} \operatorname{Hom}_{\Lambda}\left(Q_{d e g}, R\right)= \begin{cases}8 s, & \ell n+m \equiv 0(s) \\ 0, & \text { otherwise }\end{cases}
$$

(5) If $r=4$, then

$$
\operatorname{dim}_{K} \operatorname{Hom}_{\Lambda}\left(Q_{d e g}, R\right)= \begin{cases}2 s, & \ell(n+s)+m \equiv 0(2 s) \\ 6 s, & \ell(n+s)+m \equiv s(2 s) \\ 5 s, & \ell(n+s)+m \equiv 1(2 s) \\ 7 s, & \ell(n+s)+m \equiv s+1(2 s) \\ 0, & \text { otherwise. }\end{cases}
$$

(6) If $r=6$, then

$$
\operatorname{dim}_{K} \operatorname{Hom}_{\Lambda}\left(Q_{d e g}, R\right)= \begin{cases}7 s, & \ell(n+s)+m \equiv 0(2 s) \\ 5 s, & \ell(n+s)+m \equiv s(2 s) \\ 6 s, & \ell(n+s)+m \equiv 1(2 s) \\ 2 s, & \ell(n+s)+m \equiv s+1(2 s) \\ 0, & \text { otherwise. }\end{cases}
$$

(7) If $r=9$, then

$$
\operatorname{dim}_{K} \operatorname{Hom}_{\Lambda}\left(Q_{\text {deg }}, R\right)= \begin{cases}5 s, & \ell(n+s)+m \equiv 0(2 s) \\ 7 s, & \ell(n+s)+m \equiv s(2 s) \\ 0, & \text { otherwise. }\end{cases}
$$

(8) If $r=10$, then

$$
\operatorname{dim}_{K} \operatorname{Hom}_{\Lambda}\left(Q_{d e g}, R\right)= \begin{cases}2 s, & \ell(n+s)+m \equiv 0(2 s) \text { or } \ell(n+s)+m \equiv 1(2 s) \\ 6 s, & \ell(n+s)+m \equiv s(2 s) \text { or } \ell(n+s)+m \equiv s+1(2 s) \\ 0, & \text { otherwise. }\end{cases}
$$

Proof. The dimension $\operatorname{dim}_{K} \operatorname{Hom}_{\Lambda}\left(P_{i, j}, R\right)$ is equal to the number of linear independent nonzero paths of the quiver $\mathcal{Q}_{s}$, leading from $j$ th vertex to $i$ th, and the proof is to consider cases $r=0$, $r=1$ etc.

Proposition 13 (Dimensions of homomorphism groups, $s=1$ ). Let $R=R_{1}^{\prime}$ is algebra of the type $E_{6}$. Next, $t \in \mathbb{N} \cup\{0\}$, $\ell$ be the aliquot, and $r$ be the residue of division of $t$ by 11 .

(1) If $r \in\{0,3,5,7,10\}$, then $\operatorname{dim}_{K} \operatorname{Hom}_{\Lambda}\left(Q_{\text {deg }}, R\right)=8$.

(2) If $r=1$, then

$$
\operatorname{dim}_{K} \operatorname{Hom}_{\Lambda}\left(Q_{\text {deg }}, R\right)= \begin{cases}7, & \ell \vdots 2 \\ 5 & \ell \% 2\end{cases}
$$


(3) If $r=2$, then

$$
\operatorname{dim}_{K} \operatorname{Hom}_{\Lambda}\left(Q_{\text {deg }}, R\right)= \begin{cases}2, & \ell \vdots 2 \\ 6 & \ell \% 2\end{cases}
$$

(4) If $r=4$, then

$$
\operatorname{dim}_{K} \operatorname{Hom}_{\Lambda}\left(Q_{d e g}, R\right)= \begin{cases}9, & \ell \vdots 2 \\ 11 & \ell \% 2\end{cases}
$$

(5) If $r=6$, then

$$
\operatorname{dim}_{K} \operatorname{Hom}_{\Lambda}\left(Q_{d e g}, R\right)= \begin{cases}11, & \ell \vdots 2 \\ 9 & \ell \% 2\end{cases}
$$

(6) If $r=8$, then

$$
\operatorname{dim}_{K} \operatorname{Hom}_{\Lambda}\left(Q_{\text {deg }}, R\right)= \begin{cases}6, & \ell \vdots 2 \\ 2 & \ell \% 2\end{cases}
$$

(7) If $r=9$, then

$$
\operatorname{dim}_{K} \operatorname{Hom}_{\Lambda}\left(Q_{d e g}, R\right)= \begin{cases}5, & \ell \vdots 2 \\ 7 & \ell \% 2\end{cases}
$$

Proof. The proof is basically the same as proof of proposition 12 ,

Proposition 14 (Dimensions of coboundaries groups). Let $R=R_{s}^{\prime}$ is algebra of the type $E_{6}$, and let

$$
0 \longrightarrow \operatorname{Hom}_{\Lambda}\left(Q_{0}, R\right) \stackrel{\delta^{0}}{\longrightarrow} \operatorname{Hom}_{\Lambda}\left(Q_{1}, R\right) \stackrel{\delta^{1}}{\longrightarrow} \operatorname{Hom}_{\Lambda}\left(Q_{2}, R\right) \stackrel{\delta^{2}}{\longrightarrow} \ldots
$$

be a complex, obtained from minimal projective resolution (耳) of algebra $R$, by applying functor $\operatorname{Hom}_{\Lambda}(-, R)$.

Consider coboundaries groups $\operatorname{Im} \delta^{s}$ of the complex $(\mathbb{X})$. Let $\ell$ be the aliquot, and $r$ be the residue of division of $t$ by $11, m$ be the aliquot of division of $r$ by 2 . Then:

(1) If $r=0$,

$$
\operatorname{dim}_{K} \operatorname{Im} \delta^{s}=\left\{\begin{array}{l}
6 s-1, \quad \ell(n+s)+m \equiv 0(2 s), \quad \ell \vdots 2 \text { or char } K=2 \\
6 s, \quad \ell(n+s)+m \equiv 0(2 s), \quad \ell \% 2, \text { char } K \neq 2 \\
2 s, \quad \ell(n+s)+m \equiv s(2 s) \\
0, \quad \text { otherwise. }
\end{array}\right.
$$

(2) If $r=1$,

$$
\operatorname{dim}_{K} \operatorname{Im} \delta^{s}=\left\{\begin{array}{l}
s, \quad \ell(n+s)+m \equiv 0(2 s) \\
3 s-1, \quad \ell(n+s)+m \equiv s(2 s), \quad \ell \% 2 \text { or char } K=2 \\
3 s, \quad \ell(n+s)+m \equiv s(2 s), \ell \vdots 2, \text { char } K \neq 2 \\
0, \quad \text { otherwise. }
\end{array}\right.
$$


HOCHSCHILD COHOMOLOGY RING FOR SELF-INJECTIVE ALGEBRAS OF TREE CLASS $E_{6}$. II. 23

(3) If $r=2$,

$$
\operatorname{dim}_{K} \operatorname{Im} \delta^{s}= \begin{cases}3 s, & \ell(n+s)+m \equiv 0(2 s) \\ s, & \ell(n+s)+m \equiv s(2 s) \\ 0, & \text { otherwise. }\end{cases}
$$

(4) If $r=3$,

$$
\operatorname{dim}_{K} \operatorname{Im} \delta^{s}=\left\{\begin{array}{l}
5 s-1, \quad \ell(n+s)+m \equiv 0(2 s) \\
7 s-1, \quad \ell(n+s)+m \equiv s(2 s), \text { char } K=2 \\
7 s, \quad \ell(n+s)+m \equiv s(2 s), \text { char } K \neq 2 ; \\
0, \quad \text { otherwise. }
\end{array}\right.
$$

(5) If $r=4$,

$$
\operatorname{dim}_{K} \operatorname{Im} \delta^{s}=\left\{\begin{array}{l}
2 s, \quad \ell(n+s)+m \equiv 0(2 s) \\
6 s-1, \quad \ell(n+s)+m \equiv s(2 s), \quad \ell \% 2, \text { char } K=3 \\
6 s, \quad \ell(n+s)+m \equiv s(2 s), \quad \ell \vdots 2 \text { or char } K \neq 3 \\
0, \quad \text { otherwise. }
\end{array}\right.
$$

(6) If $r=5$,

$$
\operatorname{dim}_{K} \operatorname{Im} \delta^{s}=\left\{\begin{array}{l}
6 s-1, \quad \ell(n+s)+m \equiv 0(2 s), \quad \ell: 2, \text { char } K=3 \\
6 s, \quad \ell(n+s)+m \equiv 0(2 s), \quad \ell \% 2 \text { or char } K \neq 3 \\
2 s, \quad \ell(n+s)+m \equiv s(2 s) \\
0, \quad \text { otherwise. }
\end{array}\right.
$$

(7) If $r=6$,

$$
\operatorname{dim}_{K} \operatorname{Im} \delta^{s}=\left\{\begin{array}{l}
7 s-1, \quad \ell(n+s)+m \equiv 0(2 s), \text { char } K=2 \\
7 s, \quad \ell(n+s)+m \equiv 0(2 s), \text { char } K \neq 2 \\
5 s-1, \quad \ell(n+s)+m \equiv s(2 s) \\
0, \quad \text { otherwise. }
\end{array}\right.
$$

(8) If $r=7$,

$$
\operatorname{dim}_{K} \operatorname{Im} \delta^{s}= \begin{cases}s, & \ell(n+s)+m \equiv 0(2 s) \\ 3 s, & \ell(n+s)+m \equiv s(2 s) \\ 0, & \text { otherwise. }\end{cases}
$$


(9) If $r=8$,

$$
\operatorname{dim}_{K} \operatorname{Im} \delta^{s}=\left\{\begin{array}{l}
3 s-1, \quad \ell(n+s)+m \equiv 0(2 s), \quad \ell \vdots 2 \text { or char } K=2 \\
3 s, \quad \ell(n+s)+m \equiv 0(2 s), \quad \ell \% 2, \text { char } K \neq 2 \\
s, \quad \ell(n+s)+m \equiv s(2 s) \\
0, \quad \text { otherwise. }
\end{array}\right.
$$

(10) If $r=9$,

$$
\operatorname{dim}_{K} \operatorname{Im} \delta^{s}=\left\{\begin{array}{l}
2 s, \quad \ell(n+s)+m \equiv 0(2 s) \\
6 s-1, \quad \ell(n+s)+m \equiv s(2 s), \quad \ell \% 2 \text { or char } K=2 \\
6 s, \quad \ell(n+s)+m \equiv s(2 s), \quad \ell: 2, \text { char } K \neq 2 \\
0, \quad \text { otherwise. }
\end{array}\right.
$$

(11) If $r=10$,

$$
\operatorname{dim}_{K} \operatorname{Im} \delta^{s}=\left\{\begin{array}{l}
2 s-1, \quad \ell(n+s)+m \equiv 0(2 s), \ell \% 2, \text { char } K=3 \\
2 s, \quad \ell(n+s)+m \equiv 0(2 s), \ell \vdots 2 \text { or char } K \neq 3 \\
6 s, \quad \ell(n+s)+m \equiv s(2 s) \\
0, \quad \text { otherwise. }
\end{array}\right.
$$

Proof. The proof is technical and consists in constructing the image matrixes from the description of differential matrixes and the subsequent computations of the ranks of image matrixes.

Theorem 15 (Additive structure, $s>1$ ). Let $s>1$ and $R=R_{s}^{\prime}$ is algebra of the type $E_{6}$. Next, $t \in \mathbb{N} \cup\{0\}$, $\ell$ be the aliquot, and $r$ be the residue of division of $t$ by $11, m$ be the aliquot of division of $r$ by 2. Then $\operatorname{dim}_{K} \mathrm{HH}^{t}(R)=1$, if one of the following conditions takes place:

(1) $r \in\{0,1,8,9\}, \ell(n+s)+m \equiv 0(2 s), \ell \vdots 2$ or char $K=2$;

(2) $r=0, \ell(n+s)+m \equiv s+1(2 s), \ell \vdots 2, \operatorname{char} K=3$;

(3) $r \in\{1,9\}, \ell(n+s)+m \equiv s(2 s), \ell \% 2$ or char $K=2$;

(4) $r \in\{2,10\}, \ell(n+s)+m \equiv s+1(2 s), \ell \% 2$ or $\operatorname{char} K=2$;

(5) $r=3, \ell(n+s)+m \equiv 0(2 s)$;

(6) $r=3, \ell(n+s)+m \equiv s(2 s)$, $\operatorname{char} K=2$;

(7) $r \in\{4,5\}, \ell(n+s)+m \equiv s(2 s), \ell \% 2$, char $K=3$;

(8) $r=4, \ell(n+s)+m \equiv 1(2 s)$;

(9) $r=4, \ell(n+s)+m \equiv s+1(2 s)$, char $K=2$;

(10) $r=5, \ell(n+s)+m \equiv 0(2 s), \ell \vdots 2, \operatorname{char} K=3$;

(11) $r \in\{6,7\}, \ell(n+s)+m \equiv 0(2 s)$, char $K=2$;

(12) $r \in\{6,7\}, \ell(n+s)+m \equiv s(2 s)$;

(13) $r=6, \ell(n+s)+m \equiv 1(2 s), \ell \vdots 2, \operatorname{char} K=3$;

(14) $r=10, \ell(n+s)+m \equiv 0(2 s), \ell \% 2$, char $K=3$.

In other cases $\operatorname{dim}_{K} \mathrm{HH}^{t}(R)=0$. 


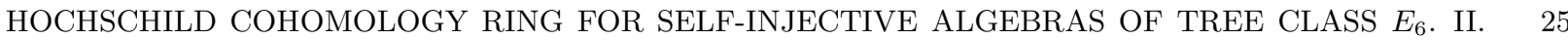

Proof. As $\operatorname{dim}_{K} \operatorname{HH}^{t}(R)=\operatorname{dim}_{K} \operatorname{Ker} \delta^{t}-\operatorname{dim}_{K} \operatorname{Im} \delta^{t-1}$, and $\operatorname{dim}_{K} \operatorname{Ker} \delta^{t}=\operatorname{dim}_{K} \operatorname{Hom}_{\Lambda}\left(Q_{t}, R\right)-$ $\operatorname{dim}_{K} \operatorname{Im} \delta^{t}$, the assertions of theorem easily follows from propositions 12 - 14.

Theorem 16 (Additive structure, $s=1$ ). Let $R=R_{1}^{\prime}$ is algebra of the type $E_{6}$. Next, $t \in \mathbb{N} \cup\{0\}$, $\ell$ be the aliquot, and $r$ be the residue of division of $t$ by 11 .

(a) $\operatorname{dim}_{K} \mathrm{HH}^{t}(R)=3$, if $t=0$.

(b) $\operatorname{dim}_{K} \mathrm{HH}^{t}(R)=2$, if one of the following conditions takes place:

(1) $r \in\{0,10\}, t>0, \ell+m \vdots 2$, char $K=3$;

(2) $r \in\{4,6\}, \ell+m \% 2$, char $K=3$.

(c) $\operatorname{dim}_{K} \mathrm{HH}^{t}(R)=1$, if one of the following conditions takes place:

(1) $r \in\{0,10\}, t>0, \ell+m \vdots 2$, char $K \neq 3$;

(2) $r \in\{1,9\}$;

(3) $r \in\{2,8\}, \ell+m \vdots 2$;

(4) $r=3, \ell+m \vdots 2$ or $\operatorname{char} K=2$;

(5) $r \in\{4,6\}, \ell+m \vdots 2$, char $K=2$;

(6) $r \in\{4,6\}, \ell+m \% 2$, char $K \neq 3$;

(7) $r=5$, char $K=3$;

(8) $r=7, \ell+m \% 2$ or $\operatorname{char} K=2$.

(d) In other cases $\operatorname{dim}_{K} \mathrm{HH}^{t}(R)=0$.

\section{Generators of $\operatorname{HH}^{*}(R)$}

For $s>1$ introduce the set of generators $Y_{t}^{(1)}, Y_{t}^{(2)}, \ldots Y_{t}^{(22)}$, such that $\operatorname{deg} Y_{t}^{(i)}=t, 0 \leqslant t<$ $11 \operatorname{deg} \sigma$ and $t$ satisfies conditions of (i)th item from the list on page 3. For $s=1$ introduce the set of generators $Y_{t}^{(1)}, Y_{t}^{(2)}, \ldots Y_{t}^{(24)}$, such that $\operatorname{deg} Y_{t}^{(i)}=t, 0 \leqslant t<11 \operatorname{deg} \sigma$ and $t$ satisfies conditions of (i)th item from the list on page 3 for $i \leqslant 22$ and $t=0$ if $i>22$. Now let us describe the matrixes of $Y_{t}^{(i)}$ componentwisely.

Denotation. Let us represent the degree $t$ of the generator element in the form $t=11 \ell+r$ $(0 \leqslant r \leqslant 10)$. Define the function $\kappa:\left\{w \in K\left[\mathcal{Q}_{s}\right]\right\} \rightarrow \mathbb{Z}$ which returns a coefficient of $\sigma(w)$; $\kappa^{\ell}(w)$ returns a coefficient of $\sigma^{\ell}(w)$.

(1) $Y_{t}^{(1)}$ is an $(6 s \times 6 s)$-matrix, whose elements $y_{i j}$ have the following form:

If $0 \leqslant j<s$, then

$$
y_{i j}=\left\{\begin{array}{l}
\kappa^{\ell}\left(\alpha_{3(j+m)}\right) e_{4 j} \otimes e_{4 j}, \quad i=j \\
0, \quad \text { otherwise }
\end{array}\right.
$$

If $s \leqslant j<3 s$, then

$$
y_{i j}=\left\{\begin{array}{l}
e_{4(j+s)+1} \otimes e_{4(j+s)+1}, \quad i=j \\
0, \quad \text { otherwise }
\end{array}\right.
$$

If $3 s \leqslant j<5 s$, then

$$
y_{i j}=\left\{\begin{array}{l}
e_{4(j+s)+2} \otimes e_{4(j+s)+2}, \quad i=j \\
0, \quad \text { otherwise. }
\end{array}\right.
$$


If $5 s \leqslant j<6 s$, then

$$
y_{i j}=\left\{\begin{array}{l}
\kappa^{\ell}\left(\alpha_{3(j+m)}\right) e_{4 j+3} \otimes e_{4 j+3}, \quad i=j \\
0, \quad \text { otherwise. }
\end{array}\right.
$$

(2) $Y_{t}^{(2)}$ is an $(6 s \times 6 s)$-matrix with a single nonzero element:

$$
y_{0,0}=\kappa^{\ell}\left(\alpha_{0}\right) w_{0 \rightarrow 4} \otimes e_{0} .
$$

(3) $Y_{t}^{(3)}$ is an $(7 s \times 6 s)$-matrix with two nonzero elements:

$$
y_{0,0}=w_{0 \rightarrow 1} \otimes e_{0} \text { and } y_{0, s}=w_{0 \rightarrow 4 s+1} \otimes e_{0} .
$$

(4) $Y_{t}^{(4)}$ is an $(7 s \times 6 s)$-matrix, whose elements $y_{i j}$ have the following form: If $0 \leqslant j<s$, then

$$
y_{i j}=\left\{\begin{array}{l}
w_{4 j \rightarrow 4(j+s)+1} \otimes e_{4 j}, \quad i=j \\
0, \quad \text { otherwise }
\end{array}\right.
$$

If $s \leqslant j<5 s-1$, then $y_{i j}=0$.

If $5 s-1 \leqslant j<6 s-1$, then

$$
y_{i j}=\left\{\begin{array}{l}
-\kappa^{\ell}\left(\alpha_{3(j+m)}\right) w_{4 j+2 \rightarrow 4 j+3} \otimes e_{4 j+2}, \quad i=j-s \\
0, \quad \text { otherwise. }
\end{array}\right.
$$

If $6 s-1 \leqslant j<7 s$, then $y_{i j}=0$.

(5) $Y_{t}^{(5)}$ is an $(6 s \times 6 s)$-matrix with a single nonzero element:

$$
y_{0,0}=\kappa^{\ell}\left(\alpha_{0}\right) w_{0 \rightarrow 3} \otimes e_{0} .
$$

(6) $Y_{t}^{(6)}$ is an $(8 s \times 6 s)$-matrix, whose elements $y_{i j}$ have the following form:

If $0 \leqslant j<s$, then

$$
y_{i j}=\left\{\begin{array}{l}
w_{4 j \rightarrow 4 j+2} \otimes e_{4 j}, \quad i=j \\
0, \quad \text { otherwise }
\end{array}\right.
$$

If $s \leqslant j<3 s$, then $y_{i j}=0$.

If $3 s \leqslant j<4 s$, then

$$
y_{i j}=\left\{\begin{array}{l}
-\kappa^{\ell}\left(\alpha_{3(j+m)}\right) w_{4 j+1 \rightarrow 4 j+3} \otimes e_{4 j+1}, \quad i=j-s \\
0, \quad \text { otherwise. }
\end{array}\right.
$$

If $4 s \leqslant j<6 s$, then

$$
y_{i j}=\left\{\begin{array}{l}
-\kappa^{\ell}\left(\alpha_{3(j+m+1)}\right) w_{4 j+2 \rightarrow 4(j+1)} \otimes e_{4 j+2}, \quad i=j-s, j<5 s-1 \text { or } j=6 s-1 \\
0, \quad \text { otherwise. }
\end{array}\right.
$$

If $6 s \leqslant j<8 s$, then

$$
y_{i j}=\left\{\begin{array}{l}
w_{4 j+3 \rightarrow 4(j+1)+1} \otimes e_{4 j+3}, \quad i=5 s+(j)_{s}, j<7 s-1 \text { or } j=8 s-1 ; \\
0, \quad \text { otherwise. }
\end{array}\right.
$$


(7) $Y_{t}^{(7)}$ is an $(8 s \times 6 s)$-matrix, whose elements $y_{i j}$ have the following form: If $0 \leqslant j<2 s$, then

$$
y_{i j}=\left\{\begin{array}{l}
w_{4 j \rightarrow 4(j+s)+2} \otimes e_{4 j}, \quad i=(j)_{s} \\
0, \quad \text { otherwise }
\end{array}\right.
$$

If $2 s \leqslant j<4 s$, then

$$
y_{i j}=\left\{\begin{array}{l}
w_{4 j+1 \rightarrow 4 j+3} \otimes e_{4 j+1}, \quad i=j-s \\
0, \quad \text { otherwise }
\end{array}\right.
$$

If $4 s \leqslant j<6 s$, then

$$
y_{i j}=\left\{\begin{array}{l}
w_{4 j+2 \rightarrow 4(j+1)} \otimes e_{4 j+2}, \quad i=j-s \\
0, \quad \text { otherwise. }
\end{array}\right.
$$

If $6 s \leqslant j<8 s$, then $y_{i j}=0$.

(8) $Y_{t}^{(8)}$ is an $(9 s \times 6 s)$-matrix with a single nonzero element:

$$
y_{0,0}=w_{0 \rightarrow 3} \otimes e_{0}
$$

(9) $Y_{t}^{(9)}$ is an $(9 s \times 6 s)$-matrix, whose elements $y_{i j}$ have the following form:

If $0 \leqslant j<s$, then $y_{i j}=0$.

If $s \leqslant j<2 s$, then

$$
y_{i j}=\left\{\begin{array}{l}
\kappa^{\ell}\left(\alpha_{3(j+m)}\right) e_{4 j} \otimes e_{4 j}, \quad i=j-s \\
0, \quad \text { otherwise }
\end{array}\right.
$$

If $2 s \leqslant j<4 s$, then

$$
y_{i j}=\left\{\begin{array}{l}
e_{4 j+1} \otimes e_{4 j+1}, \quad i=j-s \\
0, \quad \text { otherwise. }
\end{array}\right.
$$

If $4 s \leqslant j<5 s$, then $y_{i j}=0$.

If $5 s \leqslant j<6 s$, then

$$
y_{i j}=\left\{\begin{array}{l}
e_{4(j+s)+2} \otimes e_{4(j+s)+2}, \quad i=j-2 s \\
0, \quad \text { otherwise. }
\end{array}\right.
$$

If $6 s \leqslant j<7 s$, then $y_{i j}=0$.

If $7 s \leqslant j<8 s$, then

$$
y_{i j}=\left\{\begin{array}{l}
e_{4 j+2} \otimes e_{4 j+2}, \quad i=j-3 s \\
0, \quad \text { otherwise }
\end{array}\right.
$$

If $8 s \leqslant j<9 s$, then

$$
y_{i j}=\left\{\begin{array}{l}
-\kappa^{\ell}\left(\alpha_{3(j+m)}\right) e_{4 j+3} \otimes e_{4 j+3}, \quad i=j-3 s \\
0, \quad \text { otherwise. }
\end{array}\right.
$$


(10) $Y_{t}^{(10)}$ is an $(9 s \times 6 s)$-matrix with a single nonzero element:

$$
y_{0, s}=\kappa^{\ell}\left(\alpha_{6}\right) w_{0 \rightarrow 4} \otimes e_{0} .
$$

(11) $Y_{t}^{(11)}$ is an $(8 s \times 6 s)$-matrix, whose elements $y_{i j}$ have the following form: If $0 \leqslant j<2 s$, then

$$
y_{i j}=\left\{\begin{array}{l}
w_{4 j \rightarrow 4 j+1} \otimes e_{4 j}, \quad i=(j)_{s} \\
0, \quad \text { otherwise }
\end{array}\right.
$$

If $2 s \leqslant j<4 s$, then

$$
y_{i j}=\left\{\begin{array}{l}
\kappa^{\ell}\left(\alpha_{3(j+m+1)}\right) w_{4 j+1 \rightarrow 4(j+1)} \otimes e_{4 j+1}, \quad i=j-s \\
0, \quad \text { otherwise }
\end{array}\right.
$$

If $4 s \leqslant j<6 s$, then

$$
y_{i j}=\left\{\begin{array}{l}
-\kappa^{\ell}\left(\alpha_{3(j+m)}\right) w_{4 j+2 \rightarrow 4 j+3} \otimes e_{4 j+2}, \quad i=j-s \\
0, \quad \text { otherwise. }
\end{array}\right.
$$

If $6 s \leqslant j<8 s$, then

$$
y_{i j}=\left\{\begin{array}{l}
f_{1}(j, 7 s) w_{4 j+3 \rightarrow 4(j+1)+2} \otimes e_{4 j+3}, \quad i=5 s+(j)_{s} \\
0, \quad \text { otherwise }
\end{array}\right.
$$

(12) $Y_{t}^{(12)}$ is an $(8 s \times 6 s)$-matrix with two nonzero elements:

$$
y_{3 s, 4 s}=\kappa^{\ell}\left(\alpha_{3(j+m)}\right) w_{4 j+2 \rightarrow 4 j+3} \otimes e_{4 j+2} \text { and } y_{4 s, 5 s}=\kappa^{\ell}\left(\alpha_{3(j+m)}\right) w_{4 j+2 \rightarrow 4 j+3} \otimes e_{4 j+2} .
$$

(13) $Y_{t}^{(13)}$ is an $(9 s \times 6 s)$-matrix, whose elements $y_{i j}$ have the following form:

If $0 \leqslant j<s$, then $y_{i j}=0$.

If $s \leqslant j<2 s$, then

$$
y_{i j}=\left\{\begin{array}{l}
e_{4(j+s)+1} \otimes e_{4(j+s)+1}, \quad i=j \\
0, \quad \text { otherwise. }
\end{array}\right.
$$

If $2 s \leqslant j<3 s$, then $y_{i j}=0$.

If $3 s \leqslant j<4 s$, then

$$
y_{i j}=\left\{\begin{array}{l}
e_{4 j+1} \otimes e_{4 j+1}, \quad i=j-s \\
0, \quad \text { otherwise. }
\end{array}\right.
$$

If $4 s \leqslant j<5 s$, then $y_{i j}=0$.

If $5 s \leqslant j<7 s$, then

$$
y_{i j}=\left\{\begin{array}{l}
e_{4(j+s)+2} \otimes e_{4(j+s)+2}, \quad i=j-2 s \\
0, \quad \text { otherwise. }
\end{array}\right.
$$

If $7 s \leqslant j<8 s$, then $y_{i j}=0$. 
If $8 s \leqslant j<9 s$, then

$$
y_{i j}=\left\{\begin{array}{l}
w_{4 j+3 \rightarrow 4(j+1)} \otimes e_{4 j+3}, \quad i=j-3 s \\
0, \quad \text { otherwise }
\end{array}\right.
$$

(14) $Y_{t}^{(14)}$ is an $(9 s \times 6 s)$-matrix with a single nonzero element:

$$
y_{5 s, 7 s}=\kappa^{\ell}\left(\alpha_{3(j+m)}\right) w_{4 j+3 \rightarrow 4(j+1)+3} \otimes e_{4 j+3} .
$$

(15) $Y_{t}^{(15)}$ is an $(9 s \times 6 s)$-matrix, whose elements $y_{i j}$ have the following form: If $0 \leqslant j<s$, then

$$
y_{i j}=\left\{\begin{array}{l}
\kappa^{\ell}\left(\alpha_{3(j+m)}\right) e_{4 j} \otimes e_{4 j}, \quad i=j \\
0, \quad \text { otherwise }
\end{array}\right.
$$

If $s \leqslant j<2 s$, then $y_{i j}=0$.

If $2 s \leqslant j<3 s$, then

$$
y_{i j}=\left\{\begin{array}{l}
w_{4 j+1 \rightarrow 4 j+2} \otimes e_{4 j+1}, \quad i=j-s \\
0, \quad \text { otherwise. }
\end{array}\right.
$$

If $3 s \leqslant j<4 s$, then $y_{i j}=0$.

If $4 s \leqslant j<5 s$, then

$$
y_{i j}=\left\{\begin{array}{l}
w_{4(j+s)+1 \rightarrow 4(j+s)+2} \otimes e_{4(j+s)+1}, \quad i=j-2 s \\
0, \quad \text { otherwise. }
\end{array}\right.
$$

If $5 s \leqslant j<7 s$, then $y_{i j}=0$.

If $7 s \leqslant j<8 s$, then

$$
y_{i j}=\left\{\begin{array}{l}
\kappa^{\ell}\left(\alpha_{3(j+m)}\right) e_{4 j+3} \otimes e_{4 j+3}, \quad i=j-2 s \\
0, \quad \text { otherwise. }
\end{array}\right.
$$

(16) $Y_{t}^{(16)}$ is an $(8 s \times 6 s)$-matrix with two nonzero elements:

$$
y_{0,0}=w_{0 \rightarrow 2} \otimes e_{0} \text { and } y_{s, 2 s}=w_{1 \rightarrow 3} \otimes e_{1} .
$$

(17) $Y_{t}^{(17)}$ is an $(8 s \times 6 s)$-matrix with two nonzero elements:

$$
y_{0,0}=w_{0 \rightarrow 4 s+2} \otimes e_{0} \text { and } y_{0, s}=w_{0 \rightarrow 2} \otimes e_{0} .
$$

(18) $Y_{t}^{(18)}$ is an $(6 s \times 6 s)$-matrix, whose elements $y_{i j}$ have the following form:

If $0 \leqslant j<s$, then $y_{i j}=0$.

If $s \leqslant j<3 s$, then

$$
y_{i j}=\left\{\begin{array}{l}
w_{4(j+s)+1 \rightarrow 4(j+s)+2} \otimes e_{4(j+s)+1}, \quad i=j \\
0, \quad \text { otherwise. }
\end{array}\right.
$$

If $3 s \leqslant j<5 s$, then $y_{i j}=0$. 
If $5 s \leqslant j<6 s$, then

$$
y_{i j}=\left\{\begin{array}{l}
-\kappa^{\ell}\left(\alpha_{3(j+m+1)}\right) w_{4 j+3 \rightarrow 4(j+1)} \otimes e_{4 j+3}, \quad i=j \\
0, \quad \text { otherwise. }
\end{array}\right.
$$

(19) $Y_{t}^{(19)}$ is an $(7 s \times 6 s)$-matrix with two nonzero elements:

$y_{s, s}=\kappa^{\ell}\left(\alpha_{3(j+m+1)}\right) w_{4(j+s)+1 \rightarrow 4(j+1)} \otimes e_{4(j+s)+1}$ and $y_{2 s, 2 s}=\kappa^{\ell}\left(\alpha_{3(j+m+1)}\right) w_{4(j+s)+1 \rightarrow 4(j+1)} \otimes e_{4(j+s)+1}$.

$(20) Y_{t}^{(20)}$ is an $(7 s \times 6 s)$-matrix, whose elements $y_{i j}$ have the following form:

If $0 \leqslant j<s$, then

$$
y_{i j}=\left\{\begin{array}{l}
\kappa^{\ell}\left(\alpha_{3(j+m)}\right) w_{4 j \rightarrow 4 j+3} \otimes e_{4 j}, \quad i=j \\
0, \quad \text { otherwise }
\end{array}\right.
$$

If $s \leqslant j<2 s$, then

$$
y_{i j}=\left\{\begin{array}{l}
-\kappa^{\ell}\left(\alpha_{3(j+m+1)}\right) w_{4(j+s)+1 \rightarrow 4(j+1)} \otimes e_{4(j+s)+1}, \quad i=j ; \\
0, \quad \text { otherwise. }
\end{array}\right.
$$

If $2 s \leqslant j<3 s$, then $y_{i j}=0$.

If $3 s \leqslant j<4 s$, then

$$
y_{i j}=\left\{\begin{array}{l}
w_{4(j+s)+2 \rightarrow 4(j+s+1)+1} \otimes e_{4(j+s)+2}, \quad i=j \\
0, \quad \text { otherwise. }
\end{array}\right.
$$

If $4 s \leqslant j<6 s$, then $y_{i j}=0$.

If $6 s \leqslant j<7 s$, then

$$
y_{i j}=\left\{\begin{array}{l}
w_{4 j+3 \rightarrow 4(j+1)+2} \otimes e_{4 j+3}, \quad i=j-s \\
0, \quad \text { otherwise. }
\end{array}\right.
$$

$(21) Y_{t}^{(21)}$ is an $(6 s \times 6 s)$-matrix with a single nonzero element:

$$
y_{0,0}=-\kappa^{\ell}\left(\alpha_{15}\right) w_{0 \rightarrow 4} \otimes e_{0} .
$$

$(22) Y_{t}^{(22)}$ is an $(6 s \times 6 s)$-matrix, whose elements $y_{i j}$ have the following form:

If $0 \leqslant j<s$, then

$$
y_{i j}=\left\{\begin{array}{l}
\kappa^{\ell}\left(\alpha_{3(j+m)}\right) e_{4 j} \otimes e_{4 j}, \quad i=j \\
0, \quad \text { otherwise }
\end{array}\right.
$$

If $s \leqslant j<5 s$, then $y_{i j}=0$.

If $5 s \leqslant j<6 s$, then

$$
y_{i j}=\left\{\begin{array}{l}
-\kappa^{\ell}\left(\alpha_{3(j+m)}\right) e_{4 j+3} \otimes e_{4 j+3}, \quad i=j \\
0, \quad \text { otherwise. }
\end{array}\right.
$$

(23) $Y_{t}^{(23)}$ is an $(6 s \times 6 s)$-matrix with a single nonzero element:

$$
y_{0,0}=w_{0 \rightarrow 4} \otimes e_{0} .
$$


HOCHSCHILD COHOMOLOGY RING FOR SELF-INJECTIVE ALGEBRAS OF TREE CLASS $E_{6}$. II. 3

(24) $Y_{t}^{(24)}$ is an $(6 s \times 6 s)$-matrix with a single nonzero element:

$$
y_{5,5}=w_{3 \rightarrow 7} \otimes e_{3} .
$$

\section{6. $\Omega$-SHifts OF GENERATORS OF THE ALGEBRA HH* $(R)$}

Let $Q_{\bullet} \rightarrow R$ be the minimal projective bimodule resolution of the algebra $R$, constructed in paragraph 3. Any $t$-cocycle $f \in \operatorname{Ker} \delta^{t}$ is lifted (uniquely up to homotopy) to a chain map of complexes $\left\{\varphi_{i}: Q_{t+i} \rightarrow Q_{i}\right\}_{i \geqslant 0}$. The homomorphism $\varphi_{i}$ is called the $i$ th translate of the cocycle $f$ and will be denoted by $\Omega^{i}(f)$. For cocycles $f_{1} \in \operatorname{Ker} \delta^{t_{1}}$ and $f_{2} \in \operatorname{Ker} \delta^{t_{2}}$ we have

$$
\operatorname{cl} f_{2} \cdot \operatorname{cl} f_{1}=\operatorname{cl}\left(\Omega^{0}\left(f_{2}\right) \Omega^{t_{2}}\left(f_{1}\right)\right) .
$$

We shall now describe $\Omega$-translates for generators of the algebra $\operatorname{HH}^{*}(R)$ and then find multiplications of the generators using the formula (囵).

\section{Denotations.}

(1) For generator degree $t$ represent it in the form $t=11 \ell+r(0 \leqslant r \leqslant 10)$.

(2) For translate $\Omega^{t_{0}}$ represent $t_{0}$ in the form $t_{0}=11 \ell_{0}+r_{0}\left(0 \leqslant r_{0} \leqslant 10\right)$.

Proposition 17 (Translates for the case 1). (I) Let $r_{0} \in \mathbb{N}, r_{0}<11$. $r_{0}$-translates of the elements $Y_{t}^{(1)}$ are described by the following way.

(1) If $r_{0}=0$, then $\Omega^{0}\left(Y_{t}^{(1)}\right)$ is described with $(6 s \times 6 s)$-matrix with the following elements $b_{i j}$ : If $0 \leqslant j<s$, then

$$
b_{i j}=\left\{\begin{array}{l}
\kappa^{\ell}\left(\alpha_{3(j+m)}\right) e_{4 j} \otimes e_{4 j}, \quad i=j \\
0, \quad \text { otherwise. }
\end{array}\right.
$$

If $s \leqslant j<3 s$, then

$$
b_{i j}=\left\{\begin{array}{l}
e_{4(j+s)+1} \otimes e_{4(j+s)+1}, \quad i=j \\
0, \quad \text { otherwise. }
\end{array}\right.
$$

If $3 s \leqslant j<5 s$, then

$$
b_{i j}=\left\{\begin{array}{l}
e_{4(j+s)+2} \otimes e_{4(j+s)+2}, \quad i=j \\
0, \quad \text { otherwise. }
\end{array}\right.
$$

If $5 s \leqslant j<6 s$, then

$$
b_{i j}=\left\{\begin{array}{l}
\kappa^{\ell}\left(\alpha_{3(j+m)}\right) e_{4 j+3} \otimes e_{4 j+3}, \quad i=j ; \\
0, \quad \text { otherwise. }
\end{array}\right.
$$

(2) If $r_{0}=1$, then $\Omega^{1}\left(Y_{t}^{(1)}\right)$ is described with $(7 s \times 7 s)$-matrix with the following elements $b_{i j}$ : If $0 \leqslant j<2 s$, then

$$
b_{i j}=\left\{\begin{array}{l}
e_{4(j+m)+1} \otimes e_{4 j}, \quad i=j \\
0, \quad \text { otherwise. }
\end{array}\right.
$$

If $2 s \leqslant j<4 s$, then

$$
b_{i j}=\left\{\begin{array}{l}
e_{4(j+m)+2} \otimes e_{4 j+1}, \quad i=j \\
0, \quad \text { otherwise }
\end{array}\right.
$$


If $4 s \leqslant j<6 s$, then

$$
b_{i j}=\left\{\begin{array}{l}
\kappa^{\ell}\left(\alpha_{3(j+m)}\right) e_{4(j+m)+3} \otimes e_{4 j+2}, \quad i=j \\
0, \quad \text { otherwise }
\end{array}\right.
$$

If $6 s \leqslant j<7 s$, then

$$
b_{i j}=\left\{\begin{array}{l}
\kappa^{\ell}\left(\alpha_{3(j+m)}\right) e_{4(j+m+1)} \otimes e_{4 j+3}, \quad i=j \\
0, \quad \text { otherwise }
\end{array}\right.
$$

(3) If $r_{0}=2$, then $\Omega^{2}\left(Y_{t}^{(1)}\right)$ is described with $(6 s \times 6 s)$-matrix with the following elements $b_{i j}$ : If $0 \leqslant j<s$, then

$$
b_{i j}=\left\{\begin{array}{l}
\kappa^{\ell}\left(\alpha_{3(j+m)}\right) e_{4(j+m)-1} \otimes e_{4 j}, \quad i=j \\
0, \quad \text { otherwise }
\end{array}\right.
$$

If $s \leqslant j<3 s$, then

$$
b_{i j}=\left\{\begin{array}{l}
e_{4(j+m+s)+2} \otimes e_{4(j+s)+1}, \quad i=j \\
0, \quad \text { otherwise. }
\end{array}\right.
$$

If $3 s \leqslant j<5 s$, then

$$
b_{i j}=\left\{\begin{array}{l}
e_{4(j+m)+1} \otimes e_{4(j+s)+2}, \quad i=j \\
0, \quad \text { otherwise }
\end{array}\right.
$$

If $5 s \leqslant j<6 s$, then

$$
b_{i j}=\left\{\begin{array}{l}
\kappa^{\ell}\left(\alpha_{3(j+m)}\right) e_{4(j+m+1)} \otimes e_{4 j+3}, \quad i=j \\
0, \quad \text { otherwise. }
\end{array}\right.
$$

(4) If $r_{0}=3$, then $\Omega^{3}\left(Y_{t}^{(1)}\right)$ is described with $(8 s \times 8 s)$-matrix with the following elements $b_{i j}$ : If $0 \leqslant j<2 s$, then

$$
b_{i j}=\left\{\begin{array}{l}
e_{4(j+m)+2} \otimes e_{4 j}, \quad i=j \\
0, \quad \text { otherwise. }
\end{array}\right.
$$

If $2 s \leqslant j<4 s$, then

$$
b_{i j}=\left\{\begin{array}{l}
\kappa^{\ell}\left(\alpha_{3(j+m)}\right) e_{4(j+m)+3} \otimes e_{4 j+1}, \quad i=j \\
0, \quad \text { otherwise }
\end{array}\right.
$$

If $4 s \leqslant j<6 s$, then

$$
b_{i j}=\left\{\begin{array}{l}
\kappa^{\ell}\left(\alpha_{3(j+m)}\right) e_{4(j+m+1)} \otimes e_{4 j+2}, \quad i=j \\
0, \quad \text { otherwise }
\end{array}\right.
$$

If $6 s \leqslant j<8 s$, then

$$
b_{i j}=\left\{\begin{array}{l}
e_{4(j+m+1)+1} \otimes e_{4 j+3}, \quad i=j \\
0, \quad \text { otherwise }
\end{array}\right.
$$


HOCHSCHILD COHOMOLOGY RING FOR SELF-INJECTIVE ALGEBRAS OF TREE CLASS $E_{6}$. II. 33

(5) If $r_{0}=4$, then $\Omega^{4}\left(Y_{t}^{(1)}\right)$ is described with $(9 s \times 9 s)$-matrix with the following elements $b_{i j}$ : If $0 \leqslant j<s$, then

$$
b_{i j}=\left\{\begin{array}{l}
\kappa^{\ell}\left(\alpha_{3(j+m)}\right) e_{4(j+m)-1} \otimes e_{4 j}, \quad i=j \\
0, \quad \text { otherwise }
\end{array}\right.
$$

If $s \leqslant j<2 s$, then

$$
b_{i j}=\left\{\begin{array}{l}
\kappa^{\ell}\left(\alpha_{3(j+m)}\right) e_{4(j+m)} \otimes e_{4 j}, \quad i=j \\
0, \quad \text { otherwise. }
\end{array}\right.
$$

If $2 s \leqslant j<4 s$, then

$$
b_{i j}=\left\{\begin{array}{l}
e_{4(j+m+s)+1} \otimes e_{4 j+1}, \quad i=j \\
0, \quad \text { otherwise }
\end{array}\right.
$$

If $4 s \leqslant j<5 s$, then

$$
b_{i j}=\left\{\begin{array}{l}
e_{4(j+m)+1} \otimes e_{4 j+2}, \quad i=j \\
0, \quad \text { otherwise }
\end{array}\right.
$$

If $5 s \leqslant j<6 s$, then

$$
b_{i j}=\left\{\begin{array}{l}
e_{4(j+m)+2} \otimes e_{4(j+s)+2}, \quad i=j \\
0, \quad \text { otherwise }
\end{array}\right.
$$

If $6 s \leqslant j<7 s$, then

$$
b_{i j}=\left\{\begin{array}{l}
e_{4(j+m+s)+1} \otimes e_{4(j+s)+2}, \quad i=j \\
0, \quad \text { otherwise }
\end{array}\right.
$$

If $7 s \leqslant j<8 s$, then

$$
b_{i j}=\left\{\begin{array}{l}
e_{4(j+m+s)+2} \otimes e_{4 j+2}, \quad i=j \\
0, \quad \text { otherwise }
\end{array}\right.
$$

If $8 s \leqslant j<9 s$, then

$$
b_{i j}=\left\{\begin{array}{l}
\kappa^{\ell}\left(\alpha_{3(j+m)}\right) e_{4(j+m+1)-1} \otimes e_{4 j+3}, \quad i=j \\
0, \quad \text { otherwise }
\end{array}\right.
$$

(6) If $r_{0}=5$, then $\Omega^{5}\left(Y_{t}^{(1)}\right)$ is described with $(8 s \times 8 s)$-matrix with the following elements $b_{i j}$ : If $0 \leqslant j<2 s$, then

$$
b_{i j}=\left\{\begin{array}{l}
e_{4(j+m)+1} \otimes e_{4 j}, \quad i=j \\
0, \quad \text { otherwise }
\end{array}\right.
$$

If $2 s \leqslant j<4 s$, then

$$
b_{i j}=\left\{\begin{array}{l}
\kappa^{\ell}\left(\alpha_{3(j+m)}\right) e_{4(j+m+1)} \otimes e_{4 j+1}, \quad i=j \\
0, \quad \text { otherwise }
\end{array}\right.
$$


If $4 s \leqslant j<6 s$, then

$$
b_{i j}=\left\{\begin{array}{l}
\kappa^{\ell}\left(\alpha_{3(j+m)}\right) e_{4(j+m+s)+3} \otimes e_{4 j+2}, \quad i=j \\
0, \quad \text { otherwise }
\end{array}\right.
$$

If $6 s \leqslant j<8 s$, then

$$
b_{i j}=\left\{\begin{array}{l}
e_{4(j+m+1)+2} \otimes e_{4 j+3}, \quad i=j \\
0, \quad \text { otherwise. }
\end{array}\right.
$$

(7) If $r_{0}=6$, then $\Omega^{6}\left(Y_{t}^{(1)}\right)$ is described with $(9 s \times 9 s)$-matrix with the following elements $b_{i j}$ : If $0 \leqslant j<s$, then

$$
b_{i j}=\left\{\begin{array}{l}
\kappa^{\ell}\left(\alpha_{3(j+m)}\right) e_{4(j+m)} \otimes e_{4 j}, \quad i=j ; \\
0, \quad \text { otherwise. }
\end{array}\right.
$$

If $s \leqslant j<2 s$, then

$$
b_{i j}=\left\{\begin{array}{l}
e_{4(j+m+s)+1} \otimes e_{4(j+s)+1}, \quad i=j \\
0, \quad \text { otherwise }
\end{array}\right.
$$

If $2 s \leqslant j<3 s$, then

$$
b_{i j}=\left\{\begin{array}{l}
e_{4(j+m+s)+2} \otimes e_{4 j+1}, \quad i=j \\
0, \quad \text { otherwise }
\end{array}\right.
$$

If $3 s \leqslant j<4 s$, then

$$
b_{i j}=\left\{\begin{array}{l}
e_{4(j+m)+1} \otimes e_{4 j+1}, \quad i=j \\
0, \quad \text { otherwise. }
\end{array}\right.
$$

If $4 s \leqslant j<5 s$, then

$$
b_{i j}=\left\{\begin{array}{l}
e_{4(j+m)+2} \otimes e_{4(j+s)+1}, \quad i=j \\
0, \quad \text { otherwise }
\end{array}\right.
$$

If $5 s \leqslant j<7 s$, then

$$
b_{i j}=\left\{\begin{array}{l}
e_{4(j+m+s)+2} \otimes e_{4(j+s)+2}, \quad i=j \\
0, \quad \text { otherwise }
\end{array}\right.
$$

If $7 s \leqslant j<8 s$, then

$$
b_{i j}=\left\{\begin{array}{l}
\kappa^{\ell}\left(\alpha_{3(j+m)}\right) e_{4(j+m+1)-1} \otimes e_{4 j+3}, \quad i=j \\
0, \quad \text { otherwise }
\end{array}\right.
$$

If $8 s \leqslant j<9 s$, then

$$
b_{i j}=\left\{\begin{array}{l}
\kappa^{\ell}\left(\alpha_{3(j+m)}\right) e_{4(j+m+1)} \otimes e_{4 j+3}, \quad i=j ; \\
0, \quad \text { otherwise. }
\end{array}\right.
$$

(8) If $r_{0}=7$, then $\Omega^{7}\left(Y_{t}^{(1)}\right)$ is described with $(8 s \times 8 s)$-matrix with the following elements $b_{i j}$ : 
If $0 \leqslant j<2 s$, then

$$
b_{i j}=\left\{\begin{array}{l}
e_{4(j+m)+2} \otimes e_{4 j}, \quad i=j \\
0, \quad \text { otherwise. }
\end{array}\right.
$$

If $2 s \leqslant j<4 s$, then

$$
b_{i j}=\left\{\begin{array}{l}
\kappa^{\ell}\left(\alpha_{3(j+m)}\right) e_{4(j+m)+3} \otimes e_{4 j+1}, \quad i=j \\
0, \quad \text { otherwise }
\end{array}\right.
$$

If $4 s \leqslant j<6 s$, then

$$
b_{i j}=\left\{\begin{array}{l}
\kappa^{\ell}\left(\alpha_{3(j+m)}\right) e_{4(j+m+1)} \otimes e_{4 j+2}, \quad i=j \\
0, \quad \text { otherwise }
\end{array}\right.
$$

If $6 s \leqslant j<8 s$, then

$$
b_{i j}=\left\{\begin{array}{l}
e_{4(j+m+1)+1} \otimes e_{4 j+3}, \quad i=j \\
0, \quad \text { otherwise. }
\end{array}\right.
$$

(9) If $r_{0}=8$, then $\Omega^{8}\left(Y_{t}^{(1)}\right)$ is described with $(6 s \times 6 s)$-matrix with the following elements $b_{i j}$ : If $0 \leqslant j<s$, then

$$
b_{i j}=\left\{\begin{array}{l}
\kappa^{\ell}\left(\alpha_{3(j+m)}\right) e_{4(j+m)-1} \otimes e_{4 j}, \quad i=j \\
0, \quad \text { otherwise }
\end{array}\right.
$$

If $s \leqslant j<3 s$, then

$$
b_{i j}=\left\{\begin{array}{l}
e_{4(j+m+s)+2} \otimes e_{4(j+s)+1}, \quad i=j \\
0, \quad \text { otherwise }
\end{array}\right.
$$

If $3 s \leqslant j<5 s$, then

$$
b_{i j}=\left\{\begin{array}{l}
e_{4(j+m)+1} \otimes e_{4(j+s)+2}, \quad i=j \\
0, \quad \text { otherwise }
\end{array}\right.
$$

If $5 s \leqslant j<6 s$, then

$$
b_{i j}=\left\{\begin{array}{l}
\kappa^{\ell}\left(\alpha_{3(j+m)}\right) e_{4(j+m+1)} \otimes e_{4 j+3}, \quad i=j \\
0, \quad \text { otherwise. }
\end{array}\right.
$$

(10) If $r_{0}=9$, then $\Omega^{9}\left(Y_{t}^{(1)}\right)$ is described with $(7 s \times 7 s)$-matrix with the following elements $b_{i j}$ : If $0 \leqslant j<s$, then

$$
b_{i j}=\left\{\begin{array}{l}
\kappa^{\ell}\left(\alpha_{3(j+m)}\right) e_{4(j+m)+3} \otimes e_{4 j}, \quad i=j \\
0, \quad \text { otherwise. }
\end{array}\right.
$$

If $s \leqslant j<3 s$, then

$$
b_{i j}=\left\{\begin{array}{l}
\kappa^{\ell}\left(\alpha_{3(j+m)}\right) e_{4(j+m+1)} \otimes e_{4(j+s)+1}, \quad i=j \\
0, \quad \text { otherwise. }
\end{array}\right.
$$


If $3 s \leqslant j<5 s$, then

$$
b_{i j}=\left\{\begin{array}{l}
e_{4(j+m+1)+1} \otimes e_{4(j+s)+2}, \quad i=j \\
0, \quad \text { otherwise. }
\end{array}\right.
$$

If $5 s \leqslant j<7 s$, then

$$
b_{i j}=\left\{\begin{array}{l}
e_{4(j+m+1+s)+2} \otimes e_{4 j+3}, \quad i=j ; \\
0, \quad \text { otherwise. }
\end{array}\right.
$$

(11) If $r_{0}=10$, then $\Omega^{10}\left(Y_{t}^{(1)}\right)$ is described with $(6 s \times 6 s)$-matrix with the following elements $b_{i j}$ :

If $0 \leqslant j<s$, then

$$
b_{i j}=\left\{\begin{array}{l}
\kappa^{\ell}\left(\alpha_{3(j+m)}\right) e_{4(j+m)} \otimes e_{4 j}, \quad i=j \\
0, \quad \text { otherwise }
\end{array}\right.
$$

If $s \leqslant j<3 s$, then

$$
b_{i j}=\left\{\begin{array}{l}
e_{4(j+m)+1} \otimes e_{4(j+s)+1}, \quad i=j \\
0, \quad \text { otherwise }
\end{array}\right.
$$

If $3 s \leqslant j<5 s$, then

$$
b_{i j}=\left\{\begin{array}{l}
e_{4(j+m)+2} \otimes e_{4(j+s)+2}, \quad i=j \\
0, \quad \text { otherwise }
\end{array}\right.
$$

If $5 s \leqslant j<6 s$, then

$$
b_{i j}=\left\{\begin{array}{l}
\kappa^{\ell}\left(\alpha_{3(j+m)}\right) e_{4(j+m+s)+3} \otimes e_{4 j+3}, \quad i=j \\
0, \quad \text { otherwise }
\end{array}\right.
$$

(II) Represent an arbitrary $t_{0} \in \mathbb{N}$ in the form $t_{0}=11 \ell_{0}+r_{0}$, where $0 \leqslant r_{0} \leqslant 10$. Then $\Omega^{t_{0}}\left(Y_{t}^{(1)}\right)$ is a $\Omega^{r_{0}}\left(Y_{t}^{(1)}\right)$, whose left components twisted by $\sigma^{\ell_{0}}$.

Proposition 18 (Translates for the case 2). (I) Let $r_{0} \in \mathbb{N}, r_{0}<11$. Denote by $\kappa_{0}=$ $f_{2}(s, 1) \kappa^{\ell}\left(\alpha_{0}\right)$. Then $r_{0}$-translates of the elements $Y_{t}^{(2)}$ are described by the following way.

(1) If $r_{0}=0$, then $\Omega^{0}\left(Y_{t}^{(2)}\right)$ is described with $(6 s \times 6 s)$-matrix with one nonzero element that is of the following form:

$$
b_{0,0}=\kappa^{\ell}\left(\alpha_{0}\right) w_{4(j+m) \rightarrow 4(j+m+1)} \otimes e_{4 j} .
$$

(2) If $r_{0}=1$, then $\Omega^{1}\left(Y_{t}^{(2)}\right)$ is described with $(7 s \times 7 s)$-matrix with one nonzero element that is of the following form:

$$
b_{j, 6 s+(5)_{s}}=\kappa_{0} w_{4(j+m+1) \rightarrow 4(j+m+2)} \otimes e_{4 j+3} .
$$

(3) If $r_{0}=2$, then $\Omega^{2}\left(Y_{t}^{(2)}\right)$ is described with $(6 s \times 6 s)$-matrix with one nonzero element that is of the following form:

$$
b_{j, 5 s+(4)_{s}}=\kappa_{0} w_{4(j+m+1) \rightarrow 4(j+m+2)} \otimes e_{4 j+3} .
$$


HOCHSCHILD COHOMOLOGY RING FOR SELF-INJECTIVE ALGEBRAS OF TREE CLASS $E_{6}$. II. 37

(4) If $r_{0}=3$, then $\Omega^{3}\left(Y_{t}^{(2)}\right)$ is described with $(8 s \times 8 s)$-matrix with the following two nonzero elements:

$$
\begin{aligned}
& b_{j, 4 s+(4)_{s}}=\kappa_{0} w_{4(j+m+1) \rightarrow 4(j+m+2)} \otimes e_{4 j+2} ; \\
& b_{j, 5 s+(4)_{s}}=\kappa_{0} w_{4(j+m+1) \rightarrow 4(j+m+2)} \otimes e_{4 j+2} .
\end{aligned}
$$

(5) If $r_{0}=4$, then $\Omega^{4}\left(Y_{t}^{(2)}\right)$ is described with $(9 s \times 9 s)$-matrix with one nonzero element that is of the following form:

$$
b_{j, s+(4)_{s}}=\kappa_{0} w_{4(j+m) \rightarrow 4(j+m+1)} \otimes e_{4 j} .
$$

(6) If $r_{0}=5$, then $\Omega^{5}\left(Y_{t}^{(2)}\right)$ is described with $(8 s \times 8 s)$-matrix with the following two nonzero elements:

$$
\begin{aligned}
& b_{j, 2 s+(3)_{s}}=\kappa_{0} w_{4(j+m+1) \rightarrow 4(j+m+2)} \otimes e_{4 j+1} ; \\
& b_{j, 3 s+(3)_{s}}=\kappa_{0} w_{4(j+m+1) \rightarrow 4(j+m+2)} \otimes e_{4 j+1} .
\end{aligned}
$$

(7) If $r_{0}=6$, then $\Omega^{6}\left(Y_{t}^{(2)}\right)$ is described with $(9 s \times 9 s)$-matrix with the following two nonzero elements:

$$
\begin{gathered}
b_{j,(3)_{s}}=\kappa_{0} w_{4(j+m) \rightarrow 4(j+m+1)} \otimes e_{4 j} ; \\
b_{j, 8 s+(2)_{s}}=\kappa_{0} w_{4(j+m+1) \rightarrow 4(j+m+2)} \otimes e_{4 j+3} .
\end{gathered}
$$

(8) If $r_{0}=7$, then $\Omega^{7}\left(Y_{t}^{(2)}\right)$ is described with $(8 s \times 8 s)$-matrix with the following two nonzero elements:

$$
\begin{aligned}
& b_{j, 4 s+(2)_{s}}=\kappa_{0} w_{4(j+m+1) \rightarrow 4(j+m+2)} \otimes e_{4 j+2} ; \\
& b_{j, 5 s+(2)_{s}}=\kappa_{0} w_{4(j+m+1) \rightarrow 4(j+m+2)} \otimes e_{4 j+2} .
\end{aligned}
$$

(9) If $r_{0}=8$, then $\Omega^{8}\left(Y_{t}^{(2)}\right)$ is described with $(6 s \times 6 s)$-matrix with one nonzero element that is of the following form:

$$
b_{j, 5 s+(1)_{s}}=\kappa_{0} w_{4(j+m+1) \rightarrow 4(j+m+2)} \otimes e_{4 j+3} .
$$

(10) If $r_{0}=9$, then $\Omega^{9}\left(Y_{t}^{(2)}\right)$ is described with $(7 s \times 7 s)$-matrix with the following two nonzero elements:

$$
\begin{aligned}
b_{j, s+(1)_{s}} & =\kappa_{0} w_{4(j+m+1) \rightarrow 4(j+m+2)} \otimes e_{4(j+s)+1} ; \\
b_{j, 2 s+(1)_{s}} & =\kappa_{0} w_{4(j+m+1) \rightarrow 4(j+m+2)} \otimes e_{4(j+s)+1} .
\end{aligned}
$$

(11) If $r_{0}=10$, then $\Omega^{10}\left(Y_{t}^{(2)}\right)$ is described with $(6 s \times 6 s)$-matrix with one nonzero element that is of the following form:

$$
b_{j,(1)_{s}}=\kappa_{0} w_{4(j+m) \rightarrow 4(j+m+1)} \otimes e_{4 j} .
$$

(II) Represent an arbitrary $t_{0} \in \mathbb{N}$ in the form $t_{0}=11 \ell_{0}+r_{0}$, where $0 \leqslant r_{0} \leqslant 10$. Then $\Omega^{t_{0}}\left(Y_{t}^{(2)}\right)$ is a $\Omega^{r_{0}}\left(Y_{t}^{(2)}\right)$, whose left components twisted by $\sigma^{\ell_{0}}$.

Proposition 19 (Translates for the case 3). (I) Let $r_{0} \in \mathbb{N}, r_{0}<11$. $r_{0}$-translates of the elements $Y_{t}^{(3)}$ are described by the following way.

(1) If $r_{0}=0$, then $\Omega^{0}\left(Y_{t}^{(3)}\right)$ is described with $(7 s \times 6 s)$-matrix with the following two nonzero elements:

$$
\begin{aligned}
& b_{0,0}=w_{4 j \rightarrow 4 j+1} \otimes e_{4 j} ; \\
& b_{0, s}=w_{4 j \rightarrow 4 j+1} \otimes e_{4 j} .
\end{aligned}
$$

(2) If $r_{0}=1$, then $\Omega^{1}\left(Y_{t}^{(3)}\right)$ is described with $(6 s \times 7 s)$-matrix with the following nonzero elements:

$$
b_{(s+j+1)_{2 s}, s+(1)_{s}}=w_{4(j+m+s+1)+1 \rightarrow 4(j+m+s+1)+2} \otimes w_{4(j+s)+1 \rightarrow 4(j+1)} ;
$$




$$
\begin{gathered}
b_{2 s+(s+j+1)_{2 s}, s+(1)_{s}}=e_{4(j+m+s+1)+2} \otimes w_{4(j+s)+1 \rightarrow 4(j+s+1)+1} ; \\
b_{(s+j+1)_{2 s}, 2 s+(1)_{s}}=w_{4(j+m+s+1)+1 \rightarrow 4(j+m+s+1)+2} \otimes w_{4(j+s)+1 \rightarrow 4(j+1)} ; \\
b_{2 s+(s+j+1)_{2 s}, 2 s+(1)_{s}}=e_{4(j+m+s+1)+2} \otimes w_{4(j+s)+1 \rightarrow 4(j+s+1)+1} ; \\
b_{(j+1)_{2 s}, 3 s+(1)_{s}}=e_{4(j+m+1)+1} \otimes w_{4(j+s)+2 \rightarrow 4(j+1)} ; \\
b_{(j+1)_{2 s}, 4 s+(1)_{s}}=e_{4(j+m+1)+1} \otimes w_{4(j+s)+2 \rightarrow 4(j+1)} ; \\
b_{j+s, 5 s+(1)_{s}}=-\kappa^{\ell}\left(\alpha_{3(j+m)}\right) w_{4(j+m+1) \rightarrow 4(j+m+2)} \otimes e_{4 j+3} .
\end{gathered}
$$

(3) If $r_{0}=2$, then $\Omega^{2}\left(Y_{t}^{(3)}\right)$ is described with $(8 s \times 6 s)$-matrix with the following nonzero elements:

$$
\begin{gathered}
b_{(j+1)_{s}, 2 s+(1)_{s}}=f_{1}\left((j)_{s}, s-1\right) \kappa^{\ell}\left(\alpha_{3(j+m)}\right) e_{4(j+m)+3} \otimes w_{4 j+1 \rightarrow 4(j+1)} \\
b_{(j+1)_{s}, 3 s+(1)_{s}}=-f_{1}\left((j)_{s}, s-1\right) \kappa^{\ell}\left(\alpha_{3(j+m)}\right) e_{4(j+m)+3} \otimes w_{4 j+1 \rightarrow 4(j+1)} \\
b_{j-s, 4 s+(1)_{s}}=\kappa^{\ell}\left(\alpha_{3(j+m)}\right) w_{4(j+m+s)+1 \rightarrow 4(j+m+1)} \otimes e_{4 j+2} \\
b_{j-s, 5 s+(1)_{s}}=\kappa^{\ell}\left(\alpha_{3(j+m)}\right) w_{4(j+m+s)+1 \rightarrow 4(j+m+1)} \otimes e_{4 j+2} .
\end{gathered}
$$

(4) If $r_{0}=3$, then $\Omega^{3}\left(Y_{t}^{(3)}\right)$ is described with $(9 s \times 8 s)$-matrix with the following nonzero elements:

$$
\begin{gathered}
b_{j-s, s+(1)_{s}}=-\kappa^{\ell}\left(\alpha_{3(j+m)}\right) w_{4(j+m+s)+2 \rightarrow 4(j+m+1)} \otimes e_{4 j} ; \\
b_{j+s, s+(1)_{s}}=-\kappa^{\ell}\left(\alpha_{3(j+m)}\right) w_{4(j+m)+3 \rightarrow 4(j+m+1)} \otimes w_{4 j \rightarrow 4(j+s)+1} ; \\
b_{j, 2 s+(1)_{s}}=-w_{4(j+m)+3 \rightarrow 4(j+m+s+1)+1} \otimes e_{4 j+1} ; \\
b_{j, 3 s+(1)_{s}}=-w_{4(j+m)+3 \rightarrow 4(j+m+s+1)+1} \otimes e_{4 j+1} .
\end{gathered}
$$

(5) If $r_{0}=4$, then $\Omega^{4}\left(Y_{t}^{(3)}\right)$ is described with $(8 s \times 9 s)$-matrix with the following nonzero elements:

$$
\begin{gathered}
b_{j,(1)_{s}}=w_{4(j+m-1)+3 \rightarrow 4(j+m)+1} \otimes e_{4 j} ; \\
b_{s+(j+1)_{s}, 2 s}=f_{1}\left((j)_{s}, s-1\right) \kappa^{\ell}\left(\alpha_{3(j+m)}\right) e_{4(j+m+1)} \otimes w_{4 j+1 \rightarrow 4(j+1)} ; \\
b_{s+(j+1)_{s}, 3 s}=-f_{1}\left((j)_{s}, s-1\right) \kappa^{\ell}\left(\alpha_{3(j+m)}\right) e_{4(j+m+1)} \otimes w_{4 j+1 \rightarrow 4(j+1)} ; \\
b_{2 s+(s+j+1)_{2 s}, 6 s}=-w_{4(j+m+1)+1 \rightarrow 4(j+m+1)+2} \otimes w_{4 j+3 \rightarrow 4(j+s+1)+1} ; \\
b_{6 s+(s+j+1)_{2 s}-f(s, 1) s, 6 s}=e_{4(j+m+1)+2} \otimes w_{4 j+3 \rightarrow 4(j+s+1)+2} ; \\
b_{2 s+(s+j+1)_{2 s}, 7 s}=w_{4(j+m+1)+1 \rightarrow 4(j+m+1)+2} \otimes w_{4 j+3 \rightarrow 4(j+s+1)+1} ; \\
b_{5 s+(s+j+1)_{2 s}+f(s, 1) s, 7 s}=-e_{4(j+m+1)+2} \otimes w_{4 j+3 \rightarrow 4(j+s+1)+2} .
\end{gathered}
$$

(6) If $r_{0}=5$, then $\Omega^{5}\left(Y_{t}^{(3)}\right)$ is described with $(9 s \times 8 s)$-matrix with the following nonzero elements:

$$
\begin{gathered}
b_{(s+j+1)_{2 s}, s}=-e_{4(j+m+s+1)+1} \otimes w_{4(j+s)+1 \rightarrow 4(j+1)} ; \\
b_{(s+j+1)_{2 s}, 2 s}=w_{4(j+m+s+1)+1 \rightarrow 4(j+m+s+1)+2} \otimes w_{4 j+1 \rightarrow 4(j+1)} ; \\
b_{(j+1)_{2 s}, 3 s}=-e_{4(j+m+1)+1} \otimes w_{4 j+1 \rightarrow 4(j+1)} ; \\
b_{(j+1)_{2 s}, 4 s}=w_{4(j+m+1)+1 \rightarrow 4(j+m+1)+2} \otimes w_{4(j+s)+1 \rightarrow 4(j+1)} ; \\
b_{(s+j+1)_{2 s}, 7 s}=\kappa^{\ell}\left(\alpha_{3(j+m)}\right) w_{4(j+m+s+1)+1 \rightarrow 4(j+m+1)+3} \otimes w_{4 j+3 \rightarrow 4(j+1)} ; \\
b_{(j+1)_{2 s}, 7 s}=\kappa^{\ell}\left(\alpha_{3(j+m)}\right) w_{4(j+m+1)+1 \rightarrow 4(j+m+1)+3} \otimes w_{4 j+3 \rightarrow 4(j+1)} ; \\
b_{4 s+(s+j+1)_{2 s}, 7 s}=-\kappa^{\ell}\left(\alpha_{3(j+m)}\right) e_{4(j+m+1)+3} \otimes w_{4 j+3 \rightarrow 4(j+s+1)+2} ; \\
b_{2 s+(s+j+1)_{2 s}, 7 s}=-\kappa^{\ell}\left(\alpha_{3(j+m)}\right) e_{4(j+m+1)+3} \otimes w_{4 j+3 \rightarrow 4(j+1)+2} ;
\end{gathered}
$$


(7) If $r_{0}=6$, then $\Omega^{6}\left(Y_{t}^{(3)}\right)$ is described with $(8 s \times 9 s)$-matrix with the following nonzero elements:

$$
\begin{gathered}
b_{(j+1)_{s}, 5 s}=-\kappa^{\ell}\left(\alpha_{3(j+m)}\right) e_{4(j+m+1)} \otimes w_{4 j+2 \rightarrow 4(j+1)} ; \\
b_{(j+1)_{s}, 6 s}=w_{4(j+m+1) \rightarrow 4(j+m+1)+1} \otimes w_{4 j+3 \rightarrow 4(j+1)} ; \\
b_{s+(j+1)_{2 s}+f(s, 1) s, 6 s}=-e_{4(j+m+1)+1} \otimes w_{4 j+3 \rightarrow 4(j+1)+1} ; \\
b_{2 s+(j+1)_{2 s}-f(s, 1) s, 7 s}=-e_{4(j+m+1)+1} \otimes w_{4 j+3 \rightarrow 4(j+1)+1} .
\end{gathered}
$$

(8) If $r_{0}=7$, then $\Omega^{7}\left(Y_{t}^{(3)}\right)$ is described with $(6 s \times 8 s)$-matrix with the following nonzero elements:

$$
\begin{gathered}
b_{(j+s+1)_{2 s}, s}=-e_{4(j+m+s+1)+2} \otimes w_{4(j+s)+1 \rightarrow 4(j+1)} ; \\
b_{(j+s+1)_{2 s}, 2 s}=-e_{4(j+m+s+1)+2} \otimes w_{4(j+s)+1 \rightarrow 4(j+1)} ; \\
b_{s+(j+1)_{s}, 5 s}=\kappa^{\ell}\left(\alpha_{3(j+m)}\right) w_{4(j+m+1+f(s, 1) s)+2 \rightarrow 4(j+m+2)} \otimes w_{4 j+3 \rightarrow 4(j+1)} ; \\
b_{2 s+(j+1)_{s}, 5 s}=-\kappa^{\ell}\left(\alpha_{3(j+m)}\right) w_{4(j+m+1)+3 \rightarrow 4(j+m+2)} \otimes w_{4 j+3 \rightarrow 4(j+s+1+f(s, 1) s)+1} ; \\
b_{4 s+(j+s+1)_{2 s}, 5 s}=-\kappa^{\ell}\left(\alpha_{3(j+m)}\right) e_{4(j+m+2)} \otimes w_{4 j+3 \rightarrow 4(j+s+1)+2} ; \\
b_{4 s+(j+1)_{2 s}, 5 s}=-\kappa^{\ell}\left(\alpha_{3(j+m)}\right) e_{4(j+m+2)} \otimes w_{4 j+3 \rightarrow 4(j+1)+2} .
\end{gathered}
$$

(9) If $r_{0}=8$, then $\Omega^{8}\left(Y_{t}^{(3)}\right)$ is described with $(7 s \times 6 s)$-matrix with the following nonzero elements:

$$
\begin{gathered}
b_{(j+1)_{s}, 0}=f_{1}\left((j)_{s}, s-1\right) \kappa^{\ell}\left(\alpha_{3(j+m)}\right) e_{4(j+m)+3} \otimes w_{4 j \rightarrow 4(j+1)} \\
b_{(j+1)_{s}, s+f(s, 1) s}=-\kappa^{\ell}\left(\alpha_{3(j+m)}\right) w_{4(j+m)+3 \rightarrow 4(j+m+1)} \otimes w_{4(j+s)+1 \rightarrow 4(j+1)} \\
b_{3 s+(j+s+1)_{2 s}, 3 s}=e_{4(j+m+1)+1} \otimes w_{4(j+s)+2 \rightarrow 4(j+s+1)+2} \\
b_{(j+1)_{s}, 3 s+f(s, 1) s}=w_{4(j+m)+3 \rightarrow 4(j+m+1)+1} \otimes w_{4(j+s)+2 \rightarrow 4(j+1)} \\
b_{3 s+(j+s+1)_{2 s}, 4 s}=e_{4(j+m+1)+1} \otimes w_{4(j+s)+2 \rightarrow 4(j+s+1)+2} \\
b_{j, 5 s}=w_{4(j+m+1) \rightarrow 4(j+m+s+1)+2} \otimes e_{4 j+3} \\
b_{j-s, 6 s}=w_{4(j+m+1) \rightarrow 4(j+m+s+1)+2} \otimes e_{4 j+3} .
\end{gathered}
$$

(10) If $r_{0}=9$, then $\Omega^{9}\left(Y_{t}^{(3)}\right)$ is described with $(6 s \times 7 s)$-matrix with the following two nonzero elements:

$$
\begin{aligned}
& b_{j, 3 s}=-w_{4(j+m+1)+1 \rightarrow 4(j+m+1)+2} \otimes e_{4(j+s)+2} ; \\
& b_{j, 4 s}=-w_{4(j+m+1)+1 \rightarrow 4(j+m+1)+2} \otimes e_{4(j+s)+2} .
\end{aligned}
$$

(11) If $r_{0}=10$, then $\Omega^{10}\left(Y_{t}^{(3)}\right)$ is described with $(6 s \times 6 s)$-matrix with the following nonzero elements:

$$
\begin{aligned}
& b_{(j+1)_{s}, 0}=f_{1}\left((j)_{s}, s-1\right) \kappa^{\ell}\left(\alpha_{3(j+m)}\right) e_{4(j+m+1)} \otimes w_{4 j \rightarrow 4(j+1)} ; \\
& b_{j+3 s, 0}=-\kappa^{\ell}\left(\alpha_{3(j+m)}\right) w_{4(j+m+s)+2 \rightarrow 4(j+m+1)} \otimes w_{4 j \rightarrow 4 j+2} ; \\
& b_{j+4 s, 0}=\kappa^{\ell}\left(\alpha_{3(j+m)}\right) w_{4(j+m)+2 \rightarrow 4(j+m+1)} \otimes w_{4 j \rightarrow 4(j+s)+2} \text {; } \\
& b_{j+5 s, 0}=-\kappa^{\ell}\left(\alpha_{3(j+m)}\right) w_{4(j+m)+3 \rightarrow 4(j+m+1)} \otimes w_{4 j \rightarrow 4 j+3} ; \\
& b_{j, s}=w_{4(j+m)+1 \rightarrow 4(j+m+1)+1} \otimes e_{4(j+s)+1} ; \\
& b_{j, 2 s}=w_{4(j+m)+1 \rightarrow 4(j+m+1)+1} \otimes e_{4(j+s)+1} ; \\
& b_{3 s+(j+s+1)_{2 s}, 3 s+(s-1)_{s}}=e_{4(j+m+1)+2} \otimes w_{4(j+s)+2 \rightarrow 4(j+s+1)+2} ; \\
& b_{3 s+(j+s+1)_{2 s}, 4 s+(s-1)_{s}}=e_{4(j+m+1)+2} \otimes w_{4(j+s)+2 \rightarrow 4(j+s+1)+2} ; \\
& b_{3 s+(j+1)_{2 s}, 5 s+(s-1)_{s}}=-\kappa^{\ell}\left(\alpha_{3(j+m)}\right) w_{4(j+m+s+1)+2 \rightarrow 4(j+m+1)+3} \otimes w_{4 j+3 \rightarrow 4(j+1)+2} \text {; } \\
& b_{3 s+(j+s+1)_{2 s}, 5 s+(s-1)_{s}}=\kappa^{\ell}\left(\alpha_{3(j+m)}\right) w_{4(j+m+1)+2 \rightarrow 4(j+m+1)+3} \otimes w_{4 j+3 \rightarrow 4(j+s+1)+2} \text {; }
\end{aligned}
$$




$$
b_{5 s+(j+1)_{s}, 5 s+(s-1)_{s}}=f_{1}\left((j)_{s}, s-1\right) \kappa^{\ell}\left(\alpha_{3(j+m)}\right) e_{4(j+m+1)+3} \otimes w_{4 j+3 \rightarrow 4(j+1)+3} .
$$

(II) Represent an arbitrary $t_{0} \in \mathbb{N}$ in the form $t_{0}=11 \ell_{0}+r_{0}$, where $0 \leqslant r_{0} \leqslant 10$. Then $\Omega^{t_{0}}\left(Y_{t}^{(3)}\right)$ is a $\Omega^{r_{0}}\left(Y_{t}^{(3)}\right)$, whose left components twisted by $\sigma^{\ell_{0}}$, and coefficients multiplied by $(-1)^{\ell_{0}}$.

Proposition 20 (Translates for the case 4). (I) Let $r_{0} \in \mathbb{N}, r_{0}<11$. $r_{0}$-translates of the elements $Y_{t}^{(4)}$ are described by the following way.

(1) If $r_{0}=0$, then $\Omega^{0}\left(Y_{t}^{(4)}\right)$ is described with $(7 s \times 6 s)$-matrix with the following elements $b_{i j}$ : If $0 \leqslant j<s$, then

$$
b_{i j}=\left\{\begin{array}{l}
w_{4(j+m) \rightarrow 4(j+m+s)+1} \otimes e_{4 j}, \quad i=(j)_{s} \\
0, \quad \text { otherwise }
\end{array}\right.
$$

If $s \leqslant j<5 s-1$, then $b_{i j}=0$.

If $5 s-1 \leqslant j<6 s-1$, then

$$
b_{i j}=\left\{\begin{array}{l}
-\kappa^{\ell}\left(\alpha_{3(j+m)}\right) w_{4(j+m)+2 \rightarrow 4(j+m)+3} \otimes e_{4 j+2}, \quad i=j-s \\
0, \quad \text { otherwise. }
\end{array}\right.
$$

If $6 s-1 \leqslant j<7 s$, then $b_{i j}=0$.

(2) If $r_{0}=1$, then $\Omega^{1}\left(Y_{t}^{(4)}\right)$ is described with $(6 s \times 7 s)$-matrix with the following elements $b_{i j}$ : If $0 \leqslant j<s$, then

$$
b_{i j}=\left\{\begin{array}{l}
\kappa_{1} w_{4(j+m)+1 \rightarrow 4(j+m)+3} \otimes e_{4 j}, \quad i=j \\
\kappa_{1} w_{4(j+m)+2 \rightarrow 4(j+m)+3} \otimes w_{4 j \rightarrow 4 j+1}, \quad i=j+2 s \\
\kappa_{1} e_{4(j+m)+3} \otimes w_{4 j \rightarrow 4 j+2}, \quad i=j+4 s, \quad j<s-1 \\
-\kappa_{1} e_{4(j+m)+3} \otimes w_{4 j \rightarrow 4(j+s)+2}, \quad i=j+5 s, j<s-1 \\
0, \quad \text { otherwise, }
\end{array}\right.
$$

where $\kappa_{1}=\kappa^{\ell}\left(\alpha_{3(j+m)}\right)$.

If $s \leqslant j<2 s-1$, then

$$
b_{i j}=\left\{\begin{array}{l}
-w_{4(j+m+1)+1 \rightarrow 4(j+m+1)+2} \otimes w_{4(j+s)+1 \rightarrow 4(j+1)}, \quad i=s+(j+1)_{s} ; \\
0, \quad \text { otherwise. }
\end{array}\right.
$$

If $2 s-1 \leqslant j<3 s-1$, then $b_{i j}=0$.

If $3 s-1 \leqslant j<3 s$, then

$$
b_{i j}=\left\{\begin{array}{l}
-w_{4(j+m+1)+1 \rightarrow 4(j+m+1)+2} \otimes w_{4(j+s)+1 \rightarrow 4(j+1),} \quad i=s+(j+1)_{s} ; \\
0, \quad \text { otherwise. }
\end{array}\right.
$$

If $3 s \leqslant j<4 s-1$, then $b_{i j}=0$.

If $4 s-1 \leqslant j<4 s$, then

$$
b_{i j}=\left\{\begin{array}{l}
-w_{4(j+m)+3 \rightarrow 4(j+m+s+1)+1} \otimes e_{4(j+s)+2}, \quad i=j+s \\
-w_{4(j+m+1) \rightarrow 4(j+m+s+1)+1} \otimes w_{4(j+s)+2 \rightarrow 4 j+3}, \quad i=j+3 s \\
0, \quad \text { otherwise. }
\end{array}\right.
$$


If $4 s \leqslant j<5 s-1$, then

$$
b_{i j}=\left\{\begin{array}{l}
-w_{4(j+m)+3 \rightarrow 4(j+m+s+1)+1} \otimes e_{4(j+s)+2}, \quad i=j+s \\
-w_{4(j+m+1) \rightarrow 4(j+m+s+1)+1} \otimes w_{4(j+s)+2 \rightarrow 4 j+3}, \quad i=j+2 s \\
0, \quad \text { otherwise. }
\end{array}\right.
$$

If $5 s-1 \leqslant j<5 s$, then $b_{i j}=0$.

If $5 s \leqslant j<6 s$, then

$$
b_{i j}=\left\{\begin{array}{l}
f_{2}(j, 6 s-1) \kappa_{1} w_{4(j+m+1) \rightarrow 4(j+m+2)} \otimes e_{4 j+3}, \quad i=j+s ; \\
f_{2}(s, 1) \kappa_{1} w_{4(j+m+1)+3 \rightarrow 4(j+m+2)} \otimes w_{4 j+3 \rightarrow 4(j+s+1+s f(s, 1))+2}, \\
\quad i=4 s+(j+1)_{s}, j=5 s+(s-2)_{s} ; \\
f_{2}(s, 1) \kappa_{1} e_{4(j+m+2)} \otimes w_{4 j+3 \rightarrow 4(j+s+1)+3}, \quad i=6 s+(j+1)_{s}, j=5 s+(s-2)_{s} ; \\
0, \quad \text { otherwise, }
\end{array}\right.
$$

where $\kappa_{1}=\kappa^{\ell}\left(\alpha_{3(j+m+2)}\right)$.

(3) If $r_{0}=2$, then $\Omega^{2}\left(Y_{t}^{(4)}\right)$ is described with $(8 s \times 6 s)$-matrix with the following elements $b_{i j}$ : If $0 \leqslant j<s-1$, then

$$
b_{i j}=\left\{\begin{array}{l}
w_{4(j+m+s)+1 \rightarrow 4(j+m+s)+2} \otimes w_{4 j \rightarrow 4 j+2}, \quad i=j+3 s \\
0, \quad \text { otherwise. }
\end{array}\right.
$$

If $s-1 \leqslant j<s$, then $b_{i j}=0$.

If $s \leqslant j<2 s-1$, then

$$
b_{i j}=\left\{\begin{array}{l}
-w_{4(j+m+s)-1 \rightarrow 4(j+m+s)+2} \otimes e_{4 j}, \quad i=j-s \\
0, \quad \text { otherwise. }
\end{array}\right.
$$

If $2 s-1 \leqslant j<2 s$, then

$$
b_{i j}=\left\{\begin{array}{l}
-e_{4(j+m+s)+2} \otimes w_{4 j \rightarrow 4(j+s)+1}, \quad i=j \\
0, \quad \text { otherwise. }
\end{array}\right.
$$

If $2 s \leqslant j<3 s$, then

$$
b_{i j}=\left\{\begin{array}{l}
-\kappa^{\ell}\left(\alpha_{3(j+m)}\right) e_{4(j+m)+3} \otimes w_{4 j+1 \rightarrow 4(j+1)}, \quad i=(j+1)_{s} ; \\
-\kappa^{\ell}\left(\alpha_{3(j+m)}\right) w_{4(j+m)+2 \rightarrow 4(j+m)+3} \otimes e_{4 j+1}, \quad i=j-s, j=3 s-1 \\
0, \quad \text { otherwise. }
\end{array}\right.
$$

If $3 s \leqslant j<4 s$, then

$$
b_{i j}=\left\{\begin{array}{l}
-\kappa^{\ell}\left(\alpha_{3(j+m)}\right) e_{4(j+m)+3} \otimes w_{4 j+1 \rightarrow 4(j+1)}, \quad i=(j+1)_{s} ; \\
-\kappa^{\ell}\left(\alpha_{3(j+m)}\right) w_{4(j+m)+2 \rightarrow 4(j+m)+3} \otimes e_{4 j+1}, \quad i=j-s, j<4 s-1 ; \\
0, \quad \text { otherwise. }
\end{array}\right.
$$


If $4 s \leqslant j<5 s-1$, then

$$
b_{i j}=\left\{\begin{array}{l}
-\kappa_{1} w_{4(j+m)+3 \rightarrow 4(j+m+1)} \otimes w_{4 j+2 \rightarrow 4(j+1)}, \quad i=(j+1)_{s} \\
-\kappa_{1} w_{4(j+m+s)+1 \rightarrow 4(j+m+1)} \otimes e_{4 j+2}, \quad i=j-s \\
0, \quad \text { otherwise, }
\end{array}\right.
$$

where $\kappa_{1}=\kappa^{\ell}\left(\alpha_{3(j+m+1)}\right)$.

If $5 s-1 \leqslant j<6 s-1$, then $b_{i j}=0$.

If $6 s-1 \leqslant j<6 s$, then

$$
b_{i j}=\left\{\begin{array}{l}
\kappa_{1} w_{4(j+m)+3 \rightarrow 4(j+m+1)} \otimes w_{4 j+2 \rightarrow 4(j+1)}, \quad i=(j+1)_{s} ; \\
\kappa_{1} w_{4(j+m+s)+1 \rightarrow 4(j+m+1)} \otimes e_{4 j+2}, \quad i=j-s \\
0, \quad \text { otherwise }
\end{array}\right.
$$

where $\kappa_{1}=-\kappa^{\ell}\left(\alpha_{3(j+m+1)}\right)$.

If $6 s \leqslant j<7 s-1$, then

$$
b_{i j}=\left\{\begin{array}{l}
e_{4(j+m+s+1)+1} \otimes w_{4 j+3 \rightarrow 4(j+1)+2}, \quad i=3 s+(j+1)_{s} \\
0, \quad \text { otherwise. }
\end{array}\right.
$$

If $7 s-1 \leqslant j<8 s-1$, then $b_{i j}=0$.

If $8 s-1 \leqslant j<8 s$, then

$$
b_{i j}=\left\{\begin{array}{l}
e_{4(j+m+s+1)+1} \otimes w_{4 j+3 \rightarrow 4(j+1)+2}, \quad i=3 s+(j+1)_{s} \\
0, \quad \text { otherwise. }
\end{array}\right.
$$

(4) If $r_{0}=3$, then $\Omega^{3}\left(Y_{t}^{(4)}\right)$ is described with $(9 s \times 8 s)$-matrix with the following elements $b_{i j}$ : If $0 \leqslant j<s-1$, then

$$
b_{i j}=\left\{\begin{array}{l}
\kappa^{\ell}\left(\alpha_{3(j+m)}\right) w_{4(j+m)+2 \rightarrow 4(j+m)+3} \otimes e_{4 j}, \quad i=j \\
\kappa^{\ell}\left(\alpha_{3(j+m)}\right) e_{4(j+m)+3} \otimes w_{4 j \rightarrow 4 j+1}, \quad i=j+2 s \\
\kappa^{\ell}\left(\alpha_{3(j+m)}\right) e_{4(j+m)+3} \otimes w_{4 j \rightarrow 4(j+s)+1}, \quad i=j+3 s \\
0, \quad \text { otherwise. }
\end{array}\right.
$$

If $s-1 \leqslant j<2 s-1$, then $b_{i j}=0$.

If $2 s-1 \leqslant j<2 s$, then

$$
b_{i j}=\left\{\begin{array}{l}
\kappa^{\ell}\left(\alpha_{3(j+m+1)}\right) w_{4(j+m+s)+2 \rightarrow 4(j+m+1)} \otimes e_{4 j}, \quad i=j-s \\
0, \quad \text { otherwise. }
\end{array}\right.
$$

If $2 s \leqslant j<3 s-1$, then $b_{i j}=0$.

If $3 s-1 \leqslant j<3 s$, then

$$
b_{i j}=\left\{\begin{array}{l}
-w_{4(j+m)+3 \rightarrow 4(j+m+1)+1} \otimes e_{4 j+1}, \quad i=j ; \\
0, \quad \text { otherwise. }
\end{array}\right.
$$


If $3 s \leqslant j<4 s-1$, then

$$
b_{i j}=\left\{\begin{array}{l}
-w_{4(j+m)+3 \rightarrow 4(j+m+1)+1} \otimes e_{4 j+1}, \quad i=j \\
0, \quad \text { otherwise. }
\end{array}\right.
$$

If $4 s-1 \leqslant j<4 s$, then $b_{i j}=0$.

If $4 s \leqslant j<5 s-1$, then

$$
b_{i j}=\left\{\begin{array}{l}
-w_{4(j+m+1) \rightarrow 4(j+m+s+1)+1} \otimes e_{4 j+2}, \quad i=j \\
-e_{4(j+m+s+1)+1} \otimes w_{4 j+2 \rightarrow 4 j+3}, \quad i=j+3 s \\
0, \quad \text { otherwise. }
\end{array}\right.
$$

If $7 \leqslant j<8$, then

$$
b_{i j}=\left\{\begin{array}{l}
-e_{4(j+m+1)+2} \otimes w_{4 j+2 \rightarrow 4(j+1)}, \quad i=(j+1)_{s}, \quad s=1 \\
0, \quad \text { otherwise. }
\end{array}\right.
$$

If $6 s-2 \leqslant j<6 s-1$, then

$$
b_{i j}=\left\{\begin{array}{l}
-e_{4(j+m+s+1)+2} \otimes w_{4(j+s)+2 \rightarrow 4(j+1)}, \quad i=(j+1)_{s}, \quad s>1 \\
0, \quad \text { otherwise. }
\end{array}\right.
$$

If $7 s-1 \leqslant j<7 s$, then

$$
b_{i j}=\left\{\begin{array}{l}
-w_{4(j+m+1) \rightarrow 4(j+m+1)+1} \otimes e_{4(j+s)+2}, \quad i=j-s \\
-e_{4(j+m+1)+1} \otimes w_{4(j+s)+2 \rightarrow 4 j+3}, \quad i=j \\
0, \quad \text { otherwise. }
\end{array}\right.
$$

If $7 s \leqslant j<8 s+(s-2)_{s}$, then $b_{i j}=0$.

If $8 s+(s-2)_{s} \leqslant j<8 s+(s-2)_{s}+1$, then

$$
b_{i j}=\left\{\begin{array}{l}
\kappa^{\ell}\left(\alpha_{3(j+m)}\right) e_{4(j+m+1)+3} \otimes w_{4 j+3 \rightarrow 4(j+1+s f(s, 1))+1}, \quad i=2 s+(j+1)_{s} ; \\
0, \quad \text { otherwise. }
\end{array}\right.
$$

If $8 s+(s-2)_{s}+1 \leqslant j<9 s$, then $b_{i j}=0$.

(5) If $r_{0}=4$, then $\Omega^{4}\left(Y_{t}^{(4)}\right)$ is described with $(8 s \times 9 s)$-matrix with the following elements $b_{i j}$ : If $0 \leqslant j<s-1$, then

$$
b_{i j}=\left\{\begin{array}{l}
-e_{4(j+m+s)+1} \otimes w_{4 j \rightarrow 4 j+1}, \quad i=j+2 s \\
0, \quad \text { otherwise. }
\end{array}\right.
$$

If $s-1 \leqslant j<2 s-1$, then $b_{i j}=0$.

If $2 s-1 \leqslant j<2 s$, then

$$
b_{i j}=\left\{\begin{array}{l}
w_{4(j+m+s)-1 \rightarrow 4(j+m+s)+1} \otimes e_{4 j}, \quad i=j-s \\
-e_{4(j+m+s)+1} \otimes w_{4 j \rightarrow 4 j+1}, \quad i=j+2 s \\
0, \quad \text { otherwise. }
\end{array}\right.
$$


If $2 s \leqslant j<3 s-(2)_{s}$, then

$$
b_{i j}=\left\{\begin{array}{l}
f_{2}(s, 1) \kappa^{\ell}\left(\alpha_{3(j+m+1)}\right) e_{4(j+m+1)} \otimes w_{4 j+1 \rightarrow 4(j+1)}, \quad i=s+(j+1)_{s} ; \\
0, \quad \text { otherwise. }
\end{array}\right.
$$

If $3 s-(2)_{s} \leqslant j<4 s-(2)_{s}$, then $b_{i j}=0$.

If $4 s-(2)_{s} \leqslant j<4 s$, then

$$
b_{i j}=\left\{\begin{array}{l}
f_{2}(j, 4 s-2) \kappa^{\ell}\left(\alpha_{3(j+m+1)}\right) e_{4(j+m+1)} \otimes w_{4 j+1 \rightarrow 4(j+1)}, \quad i=s+(j+1)_{s} \\
0, \quad \text { otherwise. }
\end{array}\right.
$$

If $4 s \leqslant j<5 s-1$, then

$$
b_{i j}=\left\{\begin{array}{l}
\kappa^{\ell}\left(\alpha_{3(j+m)}\right) e_{4(j+m)+3} \otimes w_{4 j+2 \rightarrow 4(j+1)}, \quad i=(j+1)_{s} ; \\
-\kappa^{\ell}\left(\alpha_{3(j+m)}\right) w_{4(j+m+s)+2 \rightarrow 4(j+m)+3} \otimes e_{4 j+2}, \quad i=j+s \\
0, \quad \text { otherwise. }
\end{array}\right.
$$

If $5 s-1 \leqslant j<6 s-1$, then $b_{i j}=0$.

If $6 s-1 \leqslant j<6 s$, then

$$
b_{i j}=\left\{\begin{array}{l}
\kappa^{\ell}\left(\alpha_{3(j+m)}\right) e_{4(j+m)+3} \otimes w_{4 j+2 \rightarrow 4(j+1)}, \quad i=(j+1)_{s} \\
-\kappa^{\ell}\left(\alpha_{3(j+m)}\right) w_{4(j+m+s)+2 \rightarrow 4(j+m)+3} \otimes e_{4 j+2}, \quad i=j+2 s \\
0, \quad \text { otherwise. }
\end{array}\right.
$$

If $6 s \leqslant j<7 s-1$, then

$$
b_{i j}=\left\{\begin{array}{l}
-w_{4(j+m+s+1)+1 \rightarrow 4(j+m+s+1)+2} \otimes w_{4 j+3 \rightarrow 4(j+1)+1}, \quad i=2 s+(j+1)_{s} \\
e_{4(j+m+s+1)+2} \otimes w_{4 j+3 \rightarrow 4(j+1)+2}, \quad i=5 s+(j+1)_{s} \\
0, \quad \text { otherwise. }
\end{array}\right.
$$

If $7 s-1 \leqslant j<8 s-1$, then $b_{i j}=0$.

If $8 s-1 \leqslant j<8 s$, then

$$
b_{i j}=\left\{\begin{array}{l}
w_{4(j+m+s+1)+1 \rightarrow 4(j+m+s+1)+2} \otimes w_{4 j+3 \rightarrow 4(j+1)+1}, \quad i=2 s+(j+1)_{s} \\
-e_{4(j+m+s+1)+2} \otimes w_{4 j+3 \rightarrow 4(j+1)+2}, \quad i=5 s+(j+1)_{s} \\
0, \quad \text { otherwise. }
\end{array}\right.
$$

(6) If $r_{0}=5$ and $s=1$, then $\Omega^{5}\left(Y_{t}^{(4)}\right)$ is described with $(9 s \times 8 s)$-matrix with the following nonzero elements:

$$
\begin{gathered}
b_{j+3 s, 0}=-\kappa^{\ell}\left(\alpha_{3(j+m+1)}\right) e_{4(j+m)} \otimes w_{4 j \rightarrow 4(j+1)+1} \\
b_{j-s, s}=-e_{4(j+m+1)+1} \otimes w_{4(j+1)+1 \rightarrow 4 j} \\
b_{j-s, 2 s}=w_{4(j+m+1)+1 \rightarrow 4(j+m+1)+2} \otimes w_{4 j+1 \rightarrow 4 j} \\
b_{j-s, 6 s}=w_{4(j+m)+3 \rightarrow 4(j+m+1)+2} \otimes e_{4(j+1)+2} \\
b_{j, 6 s}=-e_{4(j+m+1)+2} \otimes w_{4(j+1)+2 \rightarrow 4 j+3} \\
b_{j-7 s, 7 s}=\kappa_{1} w_{4(j+m+1)+1 \rightarrow 4(j+m)+3} \otimes w_{4 j+3 \rightarrow 4 j} \\
b_{j-6 s, 7 s}=\kappa_{1} w_{4(j+m)+1 \rightarrow 4(j+m)+3} \otimes w_{4 j+3 \rightarrow 4 j}
\end{gathered}
$$




$$
\begin{gathered}
b_{j-3 s, 7 s}=-\kappa_{1} e_{4(j+m)+3} \otimes w_{4 j+3 \rightarrow 4(j+1)+2} ; \\
b_{j-2 s, 7 s}=-\kappa_{1} e_{4(j+m)+3} \otimes w_{4 j+3 \rightarrow 4 j+2},
\end{gathered}
$$

where $\kappa_{1}=-\kappa^{\ell}\left(\alpha_{3(7 s+m+1)}\right)$.

(7) If $r_{0}=5$ and $s>1$, then $\Omega^{5}\left(Y_{t}^{(4)}\right)$ is described with $(9 s \times 8 s)$-matrix with the following elements $b_{i j}$ :

If $0 \leqslant j<s$, then

$$
b_{i j}=\left\{\begin{array}{l}
\kappa_{1} e_{4(j+m+1)} \otimes w_{4 j \rightarrow 4 j+1}, \quad i=j+2 s \\
\kappa_{1} w_{4(j+m)+1 \rightarrow 4(j+m+1)} \otimes e_{4 j}, \quad i=j, \quad j=s-1 \\
\kappa_{1} w_{4(j+m+s)+1 \rightarrow 4(j+m+1)} \otimes e_{4 j}, \quad i=j+s, j=s-1 \\
-\kappa_{1} w_{4(j+m)+3 \rightarrow 4(j+m+1)} \otimes w_{4 j \rightarrow 4 j+2}, \quad i=j+4 s, j=s-1 \\
-\kappa_{1} w_{4(j+m)+3 \rightarrow 4(j+m+1)} \otimes w_{4 j \rightarrow 4(j+s)+2}, \quad i=j+5 s, j=s-1 \\
0, \quad \text { otherwise, }
\end{array}\right.
$$

where $\kappa_{1}=\kappa^{\ell}\left(\alpha_{3(j+m+1)}\right)$.

If $s \leqslant j<2 s-2$, then

$$
b_{i j}=\left\{\begin{array}{l}
-e_{4(j+m+1)+1} \otimes w_{4(j+s)+1 \rightarrow 4(j+1)}, \quad i=s+(j+1)_{s} ; \\
0, \quad \text { otherwise. }
\end{array}\right.
$$

If $2 s-2 \leqslant j<2 s$, then $b_{i j}=0$.

If $2 s \leqslant j<3 s-2$, then

$$
b_{i j}=\left\{\begin{array}{l}
w_{4(j+m+1)+1 \rightarrow 4(j+m+1)+2} \otimes w_{4 j+1 \rightarrow 4(j+1)}, \quad i=(j+1)_{s} \\
0, \quad \text { otherwise. }
\end{array}\right.
$$

If $3 s-2 \leqslant j<4 s-2$, then $b_{i j}=0$.

If $4 s-2 \leqslant j<4 s-1$, then

$$
b_{i j}=\left\{\begin{array}{l}
-e_{4(j+m+s+1)+1} \otimes w_{4 j+1 \rightarrow 4(j+1)}, \quad i=(j+1)_{s} ; \\
0, \quad \text { otherwise. }
\end{array}\right.
$$

If $4 s-1 \leqslant j<4 s$, then

$$
b_{i j}=\left\{\begin{array}{l}
-e_{4(j+m+s+1)+1} \otimes w_{4 j+1 \rightarrow 4(j+1)}, \quad i=s+(j+1)_{s} ; \\
0, \quad \text { otherwise. }
\end{array}\right.
$$

If $4 s \leqslant j<5 s-1$, then $b_{i j}=0$.

If $5 s-1 \leqslant j<5 s$, then

$$
b_{i j}=\left\{\begin{array}{l}
w_{4(j+m+s+1)+1 \rightarrow 4(j+m+s+1)+2} \otimes w_{4(j+s)+1 \rightarrow 4(j+1)}, \quad i=(j+1)_{s} \\
0, \quad \text { otherwise }
\end{array}\right.
$$

If $5 s \leqslant j<5 s-2$, then $b_{i j}=0$. 
If $5 s-2 \leqslant j<5 s-1$, then

$$
b_{i j}=\left\{\begin{array}{l}
w_{4(j+m+s+1)+1 \rightarrow 4(j+m+s+1)+2} \otimes w_{4(j+s)+1 \rightarrow 4(j+1)}, \quad i=s+(j+1)_{s} ; \\
0, \quad \text { otherwise. }
\end{array}\right.
$$

If $5 s-1 \leqslant j<5 s$, then $b_{i j}=0$.

If $5 s \leqslant j<6 s-1$, then

$$
b_{i j}=\left\{\begin{array}{l}
w_{4(j+m)+3 \rightarrow 4(j+m+1)+2} \otimes e_{4(j+s)+2}, \quad i=j-s \\
e_{4(j+m+1)+2} \otimes w_{4(j+s)+2 \rightarrow 4 j+3}, \quad i=j+2 s \\
0, \quad \text { otherwise. }
\end{array}\right.
$$

If $6 s-1 \leqslant j<7 s-1$, then $b_{i j}=0$.

If $7 s-1 \leqslant j<7 s$, then

$$
b_{i j}=\left\{\begin{array}{l}
w_{4(j+m)+3 \rightarrow 4(j+m+1)+2} \otimes e_{4(j+s)+2}, \quad i=j-s \\
-e_{4(j+m+1)+2} \otimes w_{4(j+s)+2 \rightarrow 4 j+3}, \quad i=j \\
0, \quad \text { otherwise. }
\end{array}\right.
$$

If $7 s \leqslant j<8 s-2$, then $b_{i j}=0$.

If $8 s-2 \leqslant j<8 s-1$, then

$$
b_{i j}=\left\{\begin{array}{l}
\kappa_{1} w_{4(j+m+s+1)+1 \rightarrow 4(j+m+1)+3} \otimes w_{4 j+3 \rightarrow 4(j+1)}, \quad i=(j+1)_{s} \\
\kappa_{1} w_{4(j+m+1)+1 \rightarrow 4(j+m+1)+3} \otimes w_{4 j+3 \rightarrow 4(j+1)}, \quad i=s+(j+1)_{s} \\
-\kappa_{1} e_{4(j+m+1)+3} \otimes w_{4 j+3 \rightarrow 4(j+s+1)+2}, \quad i=4 s+(j+1)_{s} \\
-\kappa_{1} e_{4(j+m+1)+3} \otimes w_{4 j+3 \rightarrow 4(j+1)+2}, \quad i=5 s+(j+1)_{s} \\
0, \quad \text { otherwise, }
\end{array}\right.
$$

where $\kappa_{1}=\kappa^{\ell}\left(\alpha_{3(j+m+1)}\right)$.

If $8 s-1 \leqslant j<8 s$, then $b_{i j}=0$.

If $8 s \leqslant j<9 s-1$, then

$$
b_{i j}=\left\{\begin{array}{l}
-\kappa^{\ell}\left(\alpha_{3(j+m+2)}\right) e_{4(j+m+2)} \otimes w_{4 j+3 \rightarrow 4(j+1)+1}, \quad i=2 s+(j+1)_{s} ; \\
0, \quad \text { otherwise. }
\end{array}\right.
$$

If $9 s-1 \leqslant j<9 s$, then $b_{i j}=0$.

(8) If $r_{0}=6$ and $s=1$, then $\Omega^{6}\left(Y_{t}^{(4)}\right)$ is described with $(8 s \times 9 s)$-matrix with the following nonzero elements:

$$
\begin{gathered}
b_{j+3 s, 0}=w_{4(j+m+1)+1 \rightarrow 4(j+m+1)+2} \otimes w_{4 j \rightarrow 4(j+1)+1} ; \\
b_{j+3 s, s}=-e_{4(j+m+1)+2} \otimes w_{4 j \rightarrow 4 j+1} ; \\
b_{j, 3 s}=\kappa^{\ell}\left(\alpha_{3(j+m)}\right) w_{4(j+m)+1 \rightarrow 4(j+m)+3} \otimes e_{4 j+1} ; \\
b_{j+s, 3 s}=-\kappa^{\ell}\left(\alpha_{3(j+m)}\right) w_{4(j+m+1)+2 \rightarrow 4(j+m)+3} \otimes e_{4 j+1} ; \\
b_{j+s, 5 s}=-\kappa^{\ell}\left(\alpha_{3(j+m)}\right) w_{4(j+m)+2 \rightarrow 4(j+m)} \otimes e_{4 j+2} ; \\
b_{j+2 s, 5 s}=\kappa^{\ell}\left(\alpha_{3(j+m)}\right) w_{4(j+m)+3 \rightarrow 4(j+m)} \otimes w_{4 j+2 \rightarrow 4 j+3} ;
\end{gathered}
$$




$$
\begin{gathered}
b_{j-6 s, 6 s}=-w_{4(j+m) \rightarrow 4(j+m)+1} \otimes w_{4 j+3 \rightarrow 4 j} ; \\
b_{j-5 s, 6 s}=e_{4(j+m)+1} \otimes w_{4 j+3 \rightarrow 4 j+1} .
\end{gathered}
$$

(9) If $r_{0}=6$ and $s>1$, then $\Omega^{6}\left(Y_{t}^{(4)}\right)$ is described with $(8 s \times 9 s)$-matrix with the following elements $b_{i j}$ :

If $0 \leqslant j<s$, then

$$
b_{i j}=\left\{\begin{array}{l}
-w_{4(j+m) \rightarrow 4(j+m+s)+2} \otimes e_{4 j}, \quad i=j ; \\
w_{4(j+m+s)+1 \rightarrow 4(j+m+s)+2} \otimes w_{4 j \rightarrow 4(j+s)+1}, \quad i=j+3 s \\
-e_{4(j+m+s)+2} \otimes w_{4 j \rightarrow 4(j+s)+2}, \quad i=j+6 s, \quad j<s-1 ; \\
0, \quad \text { otherwise. }
\end{array}\right.
$$

If $s \leqslant j<2 s$, then

$$
b_{i j}=\left\{\begin{array}{l}
w_{4(j+m+s)+1 \rightarrow 4(j+m+s)+2} \otimes w_{4 j \rightarrow 4(j+s)+1}, \quad i=j ; \\
0, \quad \text { otherwise. }
\end{array}\right.
$$

If $2 s \leqslant j<2 s-1$, then $b_{i j}=0$.

If $2 s-1 \leqslant j<2 s$, then

$$
b_{i j}=\left\{\begin{array}{l}
-e_{4(j+m+s)+2} \otimes w_{4 j \rightarrow 4(j+s)+2}, \quad i=j+4 s \\
0, \quad \text { otherwise. }
\end{array}\right.
$$

If $2 s \leqslant j<3 s-1$, then

$$
b_{i j}=\left\{\begin{array}{l}
\kappa^{\ell}\left(\alpha_{3(j+m)}\right) w_{4(j+m)+2 \rightarrow 4(j+m)+3} \otimes w_{4 j+1 \rightarrow 4 j+2}, \quad i=j+3 s \\
-\kappa^{\ell}\left(\alpha_{3(j+m)}\right) e_{4(j+m)+3} \otimes w_{4 j+1 \rightarrow 4 j+3}, \quad i=j+5 s \\
0, \quad \text { otherwise. }
\end{array}\right.
$$

If $3 s-1 \leqslant j<4 s-1$, then $b_{i j}=0$.

If $4 s-1 \leqslant j<4 s$, then

$$
b_{i j}=\left\{\begin{array}{l}
\kappa^{\ell}\left(\alpha_{3(j+m)}\right) w_{4(j+m)+2 \rightarrow 4(j+m)+3} \otimes w_{4 j+1 \rightarrow 4 j+2}, \quad i=j+3 s \\
-\kappa^{\ell}\left(\alpha_{3(j+m)}\right) e_{4(j+m)+3} \otimes w_{4 j+1 \rightarrow 4 j+3}, \quad i=j+4 s \\
0, \quad \text { otherwise. }
\end{array}\right.
$$

If $4 s \leqslant j<5 s-1$, then

$$
b_{i j}=\left\{\begin{array}{l}
\kappa_{1} e_{4(j+m+1)} \otimes w_{4 j+2 \rightarrow 4(j+1)}, \quad i=(j+1)_{s} \\
\kappa_{1} w_{4(j+m)+2 \rightarrow 4(j+m+1)} \otimes e_{4 j+2}, \quad i=j+s ; \\
-\kappa_{1} w_{4(j+m)+3 \rightarrow 4(j+m+1)} \otimes w_{4 j+2 \rightarrow 4 j+3}, \quad i=j+3 s \\
0, \quad \text { otherwise, }
\end{array}\right.
$$

where $\kappa_{1}=\kappa^{\ell}\left(\alpha_{3(j+m+1)}\right)$.

If $5 s-1 \leqslant j<6 s-1$, then $b_{i j}=0$. 
If $6 s-1 \leqslant j<6 s$, then

$$
b_{i j}=\left\{\begin{array}{l}
-\kappa^{\ell}\left(\alpha_{3(j+m)}\right) e_{4(j+m+1)} \otimes w_{4 j+2 \rightarrow 4(j+1)}, \quad i=(j+1)_{s} \\
-\kappa^{\ell}\left(\alpha_{3(j+m)}\right) w_{4(j+m)+2 \rightarrow 4(j+m+1)} \otimes e_{4 j+2}, \quad i=j+s \\
\kappa^{\ell}\left(\alpha_{3(j+m)}\right) w_{4(j+m)+3 \rightarrow 4(j+m+1)} \otimes w_{4 j+2 \rightarrow 4 j+3}, \quad i=j+2 s \\
0, \quad \text { otherwise. }
\end{array}\right.
$$

If $6 s \leqslant j<7 s-1$, then $b_{i j}=0$.

If $7 s-1 \leqslant j<7 s$, then

$$
b_{i j}=\left\{\begin{array}{l}
e_{4(j+m+s+1)+1} \otimes w_{4 j+3 \rightarrow 4(j+s+1)+1}, \quad i=s+(j+1)_{s} ; \\
0, \quad \text { otherwise. }
\end{array}\right.
$$

If $7 s \leqslant j<8 s-1$, then

$$
b_{i j}=\left\{\begin{array}{l}
e_{4(j+m+s+1)+1} \otimes w_{4 j+3 \rightarrow 4(j+s+1)+1}, \quad i=s+(j+1)_{s} ; \\
0, \quad \text { otherwise. }
\end{array}\right.
$$

If $8 s-1 \leqslant j<8 s$, then $b_{i j}=0$.

(10) If $r_{0}=7$ and $s=1$, then $\Omega^{7}\left(Y_{t}^{(4)}\right)$ is described with $(6 s \times 8 s)$-matrix with the following nonzero elements:

$$
\begin{gathered}
b_{j-s, s}=-e_{4(j+m+1)+2} \otimes w_{4(j+1)+1 \rightarrow 4 j} ; \\
b_{j+s, 2 s}=w_{4(j+m)+3 \rightarrow 4(j+m+1)+2} \otimes e_{4(j+1)+1} ; \\
b_{j+s, 4 s}=-w_{4(j+m) \rightarrow 4(j+m)+1} \otimes e_{4(j+1)+2} ; \\
b_{j+3 s, 4 s}=e_{4(j+m)+1} \otimes w_{4(j+1)+2 \rightarrow 4 j+3} ; \\
b_{j-5 s, 5 s}=\kappa_{1} w_{4(j+m+1)+2 \rightarrow 4(j+m)} \otimes w_{4 j+3 \rightarrow 4 j} ; \\
b_{j-s, 5 s}=-\kappa_{1} e_{4(j+m)} \otimes w_{4 j+3 \rightarrow 4(j+1)+2} ; \\
b_{j, 5 s}=-\kappa_{1} e_{4(j+m)} \otimes w_{4 j+3 \rightarrow 4 j+2} ;
\end{gathered}
$$

where $\kappa_{1}=-\kappa^{\ell}\left(\alpha_{3(5 s+m)}\right)$.

(11) If $r_{0}=7$ and $s>1$, then $\Omega^{7}\left(Y_{t}^{(4)}\right)$ is described with $(6 s \times 8 s)$-matrix with the following elements $b_{i j}$ :

If $0 \leqslant j<s$, then

$$
b_{i j}=\left\{\begin{array}{l}
\kappa^{\ell}\left(\alpha_{3(j+m)}\right) w_{4(j+m+s)+2 \rightarrow 4(j+m)+3} \otimes e_{4 j}, \quad i=j+s \\
-\kappa^{\ell}\left(\alpha_{3(j+m)}\right) e_{4(j+m)+3} \otimes w_{4 j \rightarrow 4 j+1}, \quad i=j+2 s \\
0, \quad \text { otherwise. }
\end{array}\right.
$$

If $s \leqslant j<3 s$, then $b_{i j}=0$.

If $3 s \leqslant j<4 s-1$, then

$$
b_{i j}=\left\{\begin{array}{l}
-w_{4(j+m+1) \rightarrow 4(j+m+s+1)+1} \otimes e_{4(j+s)+2}, \quad i=j+s \\
e_{4(j+m+s+1)+1} \otimes w_{4(j+s)+2 \rightarrow 4 j+3}, \quad i=j+3 s \\
0, \quad \text { otherwise. }
\end{array}\right.
$$

If $4 s-1 \leqslant j<5 s-1$, then $b_{i j}=0$. 
If $5 s-1 \leqslant j<5 s$, then

$$
b_{i j}=\left\{\begin{array}{l}
-w_{4(j+m+1) \rightarrow 4(j+m+s+1)+1} \otimes e_{4(j+s)+2}, \quad i=j+s \\
e_{4(j+m+s+1)+1} \otimes w_{4(j+s)+2 \rightarrow 4 j+3}, \quad i=j+3 s \\
0, \quad \text { otherwise. }
\end{array}\right.
$$

If $5 s \leqslant j<6 s-2$, then $b_{i j}=0$.

If $6 s-2 \leqslant j<6 s-1$, then

$$
b_{i j}=\left\{\begin{array}{l}
\kappa_{1} e_{4(j+m+2)} \otimes w_{4 j+3 \rightarrow 4(j+s+1)+2}, \quad i=4 s+(j+1)_{s} ; \\
\kappa_{1} e_{4(j+m+2)} \otimes w_{4 j+3 \rightarrow 4(j+1)+2}, \quad i=5 s+(j+1)_{s} ; \\
0, \quad \text { otherwise, }
\end{array}\right.
$$

where $\kappa_{1}=\kappa^{\ell}\left(\alpha_{3(j+m+2)}\right)$.

If $6 s-1 \leqslant j<6 s$, then $b_{i j}=0$.

(12) If $r_{0}=8$ and $s=1$, then $\Omega^{8}\left(Y_{t}^{(4)}\right)$ is described with $(7 s \times 6 s)$-matrix with the following nonzero elements:

$$
\begin{gathered}
b_{j+2 s, 0}=\kappa^{\ell}\left(\alpha_{3(j+m)}\right) w_{4(j+m+1)+2 \rightarrow 4(j+m)+3} \otimes w_{4 j \rightarrow 4(j+1)+1} \\
b_{j, 2 s}=\kappa^{\ell}\left(\alpha_{3(j+m)}\right) w_{4(j+m+1)+2 \rightarrow 4(j+m)} \otimes e_{4(j+1)+1} \\
b_{j-4 s, 4 s}=-w_{4(j+m)+3 \rightarrow 4(j+m)+1} \otimes w_{4(j+1)+2 \rightarrow 4 j} ; \\
b_{j+s, 4 s}=-w_{4(j+m) \rightarrow 4(j+m)+1} \otimes w_{4(j+1)+2 \rightarrow 4 j+3} ; \\
b_{j-5 s, 5 s}=-w_{4(j+m)+3 \rightarrow 4(j+m+1)+2} \otimes w_{4 j+3 \rightarrow 4 j} \\
b_{j-4 s, 5 s}=e_{4(j+m+1)+2} \otimes w_{4 j+3 \rightarrow 4(j+1)+1} \\
b_{j-s, 5 s}=w_{4(j+m+1)+1 \rightarrow 4(j+m+1)+2} \otimes w_{4 j+3 \rightarrow 4 j+2} \\
b_{j-3 s, 6 s}=-w_{4(j+m+1)+1 \rightarrow 4(j+m+1)+2} \otimes w_{4 j+3 \rightarrow 4 j+2} .
\end{gathered}
$$

(13) If $r_{0}=8$ and $s>1$, then $\Omega^{8}\left(Y_{t}^{(4)}\right)$ is described with $(7 s \times 6 s)$-matrix with the following elements $b_{i j}$ :

If $0 \leqslant j<s$, then

$$
b_{i j}=\left\{\begin{array}{l}
\kappa^{\ell}\left(\alpha_{3(j+m)}\right) e_{4(j+m)+3} \otimes w_{4 j \rightarrow 4(j+1)}, \quad i=(j+1)_{s} \\
\kappa^{\ell}\left(\alpha_{3(j+m)}\right) w_{4(j+m)+2 \rightarrow 4(j+m)+3} \otimes w_{4 j \rightarrow 4 j+1}, \quad i=j+s, j<s-1 ; \\
\kappa^{\ell}\left(\alpha_{3(j+m)}\right) w_{4(j+m-1)+3 \rightarrow 4(j+m)+3} \otimes e_{4 j}, \quad i=j, \quad j=s-1 ; \\
\kappa^{\ell}\left(\alpha_{3(j+m)}\right) w_{4(j+m+s)+2 \rightarrow 4(j+m)+3} \otimes w_{4 j \rightarrow 4(j+s)+1}, \quad i=j+2 s, j=s-1 ; \\
0, \quad \text { otherwise. }
\end{array}\right.
$$

If $s \leqslant j<2 s-1$, then

$$
b_{i j}=\left\{\begin{array}{l}
\kappa_{1} w_{4(j+m)+3 \rightarrow 4(j+m+1)} \otimes w_{4(j+s)+1 \rightarrow 4(j+1)}, \quad i=(j+1)_{s} \\
\kappa_{1} w_{4(j+m+s)+2 \rightarrow 4(j+m+1)} \otimes e_{4(j+s)+1}, \quad i=j \\
0, \quad \text { otherwise, }
\end{array}\right.
$$

where $\kappa_{1}=-\kappa^{\ell}\left(\alpha_{3(j+m+1)}\right)$.

If $2 s-1 \leqslant j<3 s-1$, then $b_{i j}=0$. 
If $3 s-1 \leqslant j<3 s$, then

$$
b_{i j}=\left\{\begin{array}{l}
-\kappa_{1} w_{4(j+m)+1 \rightarrow 4(j+m+1)} \otimes w_{4(j+s)+1 \rightarrow 4(j+s)+2}, \quad i=j+2 s \\
\kappa_{1} e_{4(j+m+1)} \otimes w_{4(j+s)+1 \rightarrow 4 j+3}, \quad i=j+3 s \\
0, \quad \text { otherwise, }
\end{array}\right.
$$

where $\kappa_{1}=\kappa^{\ell}\left(\alpha_{3(j+m+1)}\right)$.

If $3 s \leqslant j<4 s-1$, then

$$
b_{i j}=\left\{\begin{array}{l}
-w_{4(j+m+1) \rightarrow 4(j+m+s+1)+1} \otimes w_{4(j+s)+2 \rightarrow 4 j+3}, \quad i=j+2 s \\
0, \quad \text { otherwise. }
\end{array}\right.
$$

If $4 s-1 \leqslant j<5 s-1$, then $b_{i j}=0$.

If $5 s-1 \leqslant j<5 s$, then

$$
b_{i j}=\left\{\begin{array}{l}
-w_{4(j+m+1) \rightarrow 4(j+m+s+1)+1} \otimes w_{4(j+s)+2 \rightarrow 4 j+3}, \quad i=j+s \\
0, \quad \text { otherwise. }
\end{array}\right.
$$

If $5 s \leqslant j<6 s-1$, then $b_{i j}=0$.

If $6 s-1 \leqslant j<6 s$, then

$$
b_{i j}=\left\{\begin{array}{l}
e_{4(j+m+1)+2} \otimes w_{4 j+3 \rightarrow 4(j+1)+1}, \quad i=s+(j+1)_{s} \\
0, \quad \text { otherwise. }
\end{array}\right.
$$

If $6 s \leqslant j<6 s-2$, then $b_{i j}=0$.

If $6 s-2 \leqslant j<6 s-1$, then

$$
b_{i j}=\left\{\begin{array}{l}
-w_{4(j+m+1)+1 \rightarrow 4(j+m+1)+2} \otimes w_{4 j+3 \rightarrow 4(j+s+1)+2}, \quad i=3 s+(j+1)_{s} ; \\
0, \quad \text { otherwise. }
\end{array}\right.
$$

If $6 s-1 \leqslant j<6 s$, then $b_{i j}=0$.

If $6 s \leqslant j<7 s-1$, then

$$
b_{i j}=\left\{\begin{array}{l}
e_{4(j+m+1)+2} \otimes w_{4 j+3 \rightarrow 4(j+1)+1}, \quad i=s+(j+1)_{s} \\
0, \quad \text { otherwise. }
\end{array}\right.
$$

If $7 s-1 \leqslant j<7 s-2$, then $b_{i j}=0$.

If $7 s-2 \leqslant j<7 s-1$, then

$$
b_{i j}=\left\{\begin{array}{l}
w_{4(j+m+1)+1 \rightarrow 4(j+m+1)+2} \otimes w_{4 j+3 \rightarrow 4(j+s+1)+2}, \quad i=4 s+(j+1)_{s} \\
0, \quad \text { otherwise. }
\end{array}\right.
$$

If $7 s-1 \leqslant j<7 s$, then $b_{i j}=0$.

(14) If $r_{0}=9$ and $s=1$, then $\Omega^{9}\left(Y_{t}^{(4)}\right)$ is described with $(6 s \times 7 s)$-matrix with the following nonzero elements:

$$
\begin{gathered}
b_{j, 2 s}=-w_{4(j+m) \rightarrow 4(j+m)+1} \otimes e_{4(j+1)+1} \\
b_{j+2 s, 4 s}=e_{4(j+m)+2} \otimes w_{4(j+1)+2 \rightarrow 4 j+3}
\end{gathered}
$$


HOCHSCHILD COHOMOLOGY RING FOR SELF-INJECTIVE ALGEBRAS OF TREE CLASS $E_{6}$. II. 5

$$
\begin{gathered}
b_{j, 5 s}=-\kappa^{\ell}\left(\alpha_{3(j+m+1)}\right) w_{4(j+m)+2 \rightarrow 4(j+m)+3} \otimes e_{4 j+3} ; \\
b_{j+s, 5 s}=\kappa^{\ell}\left(\alpha_{3(j+m+1)}\right) w_{4(j+m+1)+2 \rightarrow 4(j+m)+3} \otimes e_{4 j+3} .
\end{gathered}
$$

(15) If $r_{0}=9$ and $s>1$, then $\Omega^{9}\left(Y_{t}^{(4)}\right)$ is described with $(6 s \times 7 s)$-matrix with the following elements $b_{i j}$ :

If $s-1 \leqslant j<s$, then

$$
b_{i j}=\left\{\begin{array}{l}
-\kappa_{1} w_{4(j+m)+3 \rightarrow 4(j+m+1)} \otimes e_{4 j}, \quad i=j ; \\
-\kappa_{1} e_{4(j+m+1)} \otimes w_{4 j \rightarrow 4 j+1}, \quad i=j+s ; \\
0, \quad \text { otherwise, }
\end{array}\right.
$$

where $\kappa_{1}=\kappa^{\ell}\left(\alpha_{3(j+m+1)}\right)$.

If $s \leqslant j<2 s-1$, then

$$
b_{i j}=\left\{\begin{array}{l}
-w_{4(j+m+1) \rightarrow 4(j+m+s+1)+1} \otimes e_{4(j+s)+1}, \quad i=j ; \\
0, \quad \text { otherwise. }
\end{array}\right.
$$

If $2 s-1 \leqslant j<3 s$, then $b_{i j}=0$.

If $3 s \leqslant j<4 s-1$, then

$$
b_{i j}=\left\{\begin{array}{l}
e_{4(j+m+s+1)+2} \otimes w_{4(j+s)+2 \rightarrow 4 j+3}, \quad i=j+2 s \\
0, \quad \text { otherwise. }
\end{array}\right.
$$

If $4 s-1 \leqslant j<5 s-1$, then $b_{i j}=0$.

If $5 s-1 \leqslant j<5 s$, then

$$
b_{i j}=\left\{\begin{array}{l}
e_{4(j+m+s+1)+2} \otimes w_{4(j+s)+2 \rightarrow 4 j+3}, \quad i=j+2 s \\
0, \quad \text { otherwise. }
\end{array}\right.
$$

If $5 s \leqslant j<6 s-2$, then $b_{i j}=0$.

If $6 s-2 \leqslant j<6 s-1$, then

$$
b_{i j}=\left\{\begin{array}{l}
\kappa^{\ell}\left(\alpha_{3(j+m)}\right) e_{4(j+m+1)+3} \otimes w_{4 j+3 \rightarrow 4(j+1)}, \quad i=(j+1)_{s} ; \\
0, \quad \text { otherwise. }
\end{array}\right.
$$

If $6 s-1 \leqslant j<6 s$, then $b_{i j}=0$.

(16) If $r_{0}=10$ and $s=1$, then $\Omega^{10}\left(Y_{t}^{(4)}\right)$ is described with $(6 s \times 6 s)$-matrix with the following nonzero elements:

$$
\begin{gathered}
b_{j+2 s, 0}=\kappa_{1} w_{4(j+m)+1 \rightarrow 4(j+m)} \otimes w_{4 j \rightarrow 4(j+1)+1} ; \\
b_{j+4 s, 0}=-\kappa_{1} w_{4(j+m)+2 \rightarrow 4(j+m)} \otimes w_{4 j \rightarrow 4(j+1)+2} ; \\
b_{j-s, s}=-w_{4(j+m) \rightarrow 4(j+m)+1} \otimes w_{4(j+1)+1 \rightarrow 4 j} ; \\
b_{j+4 s, s}=-w_{4(j+m)+3 \rightarrow 4(j+m)+1} \otimes w_{4(j+1)+1 \rightarrow 4 j+3} ; \\
b_{j-3 s, 3 s}=-w_{4(j+m) \rightarrow 4(j+m)+2} \otimes w_{4(j+1)+2 \rightarrow 4 j} ; \\
b_{j+2 s, 3 s}=-w_{4(j+m)+3 \rightarrow 4(j+m)+2} \otimes w_{4(j+1)+2 \rightarrow 4 j+3} ; \\
b_{j-3 s, 5 s}=-\kappa_{2} w_{4(j+m+1)+1 \rightarrow 4(j+m)+3} \otimes w_{4 j+3 \rightarrow 4 j+1} ; \\
b_{j-s, 5 s}=\kappa_{2} w_{4(j+m+1)+2 \rightarrow 4(j+m)+3} \otimes w_{4 j+3 \rightarrow 4 j+2} ;
\end{gathered}
$$


where $\kappa_{1}=\kappa^{\ell}\left(\alpha_{3(m+1)}\right), \kappa_{2}=\kappa^{\ell}\left(\alpha_{3(5 s+m+1)}\right)$.

(17) If $r_{0}=10$ and $s>1$, then $\Omega^{10}\left(Y_{t}^{(4)}\right)$ is described with $(6 s \times 6 s)$-matrix with the following elements $b_{i j}$ :

If $0 \leqslant j<s$, then

$$
b_{i j}=\left\{\begin{array}{l}
\kappa_{1} w_{4(j+m) \rightarrow 4(j+m+1)} \otimes e_{4 j}, \quad i=j ; \\
\kappa_{1} w_{4(j+m)+1 \rightarrow 4(j+m+1)} \otimes w_{4 j \rightarrow 4(j+s)+1}, \quad i=j+2 s \\
-\kappa_{1} w_{4(j+m)+2 \rightarrow 4(j+m+1)} \otimes w_{4 j \rightarrow 4(j+s)+2}, \quad i=j+4 s \\
\kappa_{1} e_{4(j+m+1)} \otimes w_{4 j \rightarrow 4(j+1)}, \quad i=(j+1)_{s}, \quad j=s-1 \\
\kappa_{1} w_{4(j+m)+3 \rightarrow 4(j+m+1)} \otimes w_{4 j \rightarrow 4 j+3}, \quad i=j+5 s, j=s-1 \\
0, \quad \text { otherwise, }
\end{array}\right.
$$

where $\kappa_{1}=\kappa^{\ell}\left(\alpha_{3(j+m+1)}\right)$.

If $s \leqslant j<2 s-1$, then

$$
b_{i j}= \begin{cases}w_{4(j+m+1) \rightarrow 4(j+m+s+1)+1} \otimes w_{4(j+s)+1 \rightarrow 4(j+1)}, & i=(j+1)_{s} \\ -w_{4(j+m)+3 \rightarrow 4(j+m+s+1)+1} \otimes w_{4(j+s)+1 \rightarrow 4 j+3}, & i=j+4 s \\ 0, \quad \text { otherwise. } & \end{cases}
$$

If $2 s-1 \leqslant j<3 s-1$, then $b_{i j}=0$.

If $3 s-1 \leqslant j<3 s$, then

$$
b_{i j}=\left\{\begin{array}{l}
w_{4(j+m+1) \rightarrow 4(j+m+s+1)+1} \otimes w_{4(j+s)+1 \rightarrow 4(j+1),} \quad i=(j+1)_{s} \\
w_{4(j+m)+3 \rightarrow 4(j+m+s+1)+1} \otimes w_{4(j+s)+1 \rightarrow 4 j+3}, \quad i=j+3 s \\
0, \quad \text { otherwise. }
\end{array}\right.
$$

If $3 s \leqslant j<4 s-1$, then

$$
b_{i j}= \begin{cases}w_{4(j+m+1) \rightarrow 4(j+m+s+1)+2} \otimes w_{4(j+s)+2 \rightarrow 4(j+1)}, & i=(j+1)_{s} \\ -w_{4(j+m)+3 \rightarrow 4(j+m+s+1)+2} \otimes w_{4(j+s)+2 \rightarrow 4 j+3}, & i=j+2 s \\ 0, \quad \text { otherwise. } & \end{cases}
$$

If $4 s-1 \leqslant j<5 s-1$, then $b_{i j}=0$.

If $5 s-1 \leqslant j<5 s$, then

$$
b_{i j}=\left\{\begin{array}{l}
w_{4(j+m+1) \rightarrow 4(j+m+s+1)+2} \otimes w_{4(j+s)+2 \rightarrow 4(j+1)}, \quad i=(j+1)_{s} \\
w_{4(j+m)+3 \rightarrow 4(j+m+s+1)+2} \otimes w_{4(j+s)+2 \rightarrow 4 j+3}, \quad i=j+s \\
0, \quad \text { otherwise. }
\end{array}\right.
$$


HOCHSCHILD COHOMOLOGY RING FOR SELF-INJECTIVE ALGEBRAS OF TREE CLASS $E_{6}$. II. 53 If $5 s \leqslant j<6 s$, then

$$
b_{i j}=\left\{\begin{array}{l}
-\kappa_{1} w_{4(j+m+1) \rightarrow 4(j+m+1)+3} \otimes w_{4 j+3 \rightarrow 4(j+1)}, \quad i=(j+1)_{s} ; \\
-\kappa_{1} w_{4\left(j+m+s+1+j_{0}\right)+1 \rightarrow 4(j+m+1)+3} \otimes w_{4 j+3 \rightarrow 4\left(j+1+j_{0}\right)+1}, \quad i=2 s+(j+1)_{s} ; \\
\kappa_{1} w_{4\left(j+m+s+1+j_{0}\right)+2 \rightarrow 4(j+m+1)+3} \otimes w_{4 j+3 \rightarrow 4\left(j+1+j_{0}\right)+2}, \quad i=4 s+(j+1)_{s} ; \\
-f_{2}(j, 6 s-1) \kappa_{1} w_{4(j+m)+3 \rightarrow 4(j+m+1)+3} \otimes e_{4 j+3}, \quad i=j ; \\
-\kappa_{1} e_{4(j+m+1)+3} \otimes w_{4 j+3 \rightarrow 4(j+1)+3}, \quad i=5 s+(j+1)_{s}, \quad j=6 s-2 ; \\
0, \quad \text { otherwise, }
\end{array}\right.
$$

where $\kappa_{1}=\kappa^{\ell}\left(\alpha_{3(j+m+1)}\right), j_{0}=s f(j, 6 s-1)$

(II) Represent an arbitrary $t_{0} \in \mathbb{N}$ in the form $t_{0}=11 \ell_{0}+r_{0}$, where $0 \leqslant r_{0} \leqslant 10$. Then $\Omega^{t_{0}}\left(Y_{t}^{(4)}\right)$ is a $\Omega^{r_{0}}\left(Y_{t}^{(4)}\right)$, whose left components twisted by $\sigma^{\ell_{0}}$.

Proposition 21 (Translates for the case 5). (I) Let $r_{0} \in \mathbb{N}, r_{0}<11$. Denote by $\kappa_{0}=\kappa^{\ell}\left(\alpha_{3\left((3)_{s}\right)}\right)$. Then $r_{0}$-translates of the elements $Y_{t}^{(5)}$ are described by the following way.

(1) If $r_{0}=0$, then $\Omega^{0}\left(Y_{t}^{(5)}\right)$ is described with $(6 s \times 6 s)$-matrix with one nonzero element that is of the following form:

$$
b_{0,0}=\kappa^{\ell}\left(\alpha_{0}\right) w_{4 j \rightarrow 4 j+3} \otimes e_{4 j} .
$$

(2) If $r_{0}=1$, then $\Omega^{1}\left(Y_{t}^{(5)}\right)$ is described with $(8 s \times 7 s)$-matrix with the following nonzero elements:

$$
\begin{gathered}
b_{(j+s+1)_{2 s}, 2 s+(2)_{s}}=f_{2}\left((j)_{s}, s-1\right) \kappa_{0} w_{4(j+m+s+1)+1 \rightarrow 4(j+m+1)+3} \otimes w_{4 j+1 \rightarrow 4(j+1)} ; \\
b_{(j+s+1)_{2 s}, 3 s+(2)_{s}}=-f_{2}\left((j)_{s}, s-1\right) \kappa_{0} w_{4(j+m+s+1)+1 \rightarrow 4(j+m+1)+3} \otimes w_{4 j+1 \rightarrow 4(j+1)} ; \\
b_{6 s+(j)_{s}, 4 s+(2)_{s}+s f(s, 1)+s f(s, 3)}=-\kappa^{\ell}\left(\gamma_{j+1+m}\right) \kappa_{0} w_{4(j+m+1) \rightarrow 4(j+m+2)} \otimes w_{4 j+2 \rightarrow 4 j+3} .
\end{gathered}
$$

(3) If $r_{0}=2$, then $\Omega^{2}\left(Y_{t}^{(5)}\right)$ is described with $(9 s \times 6 s)$-matrix with the following two nonzero elements:

$$
\begin{gathered}
b_{(j+1)_{s},(2)_{s}}=\kappa_{0} e_{4(j+m)+3} \otimes w_{4 j \rightarrow 4(j+1)} ; \\
b_{j+s, s+(2)_{s}}=f_{2}\left((j)_{s}, s-1\right) \kappa^{\ell}\left(\gamma_{j+m}\right) \kappa_{0} w_{4(j+m)+2 \rightarrow 4(j+m+1)} \otimes w_{4 j \rightarrow 4 j+1} .
\end{gathered}
$$

(4) If $r_{0}=3$, then $\Omega^{3}\left(Y_{t}^{(5)}\right)$ is described with $(8 s \times 8 s)$-matrix with the following two nonzero elements:

$$
\begin{gathered}
b_{j+3 s,(2)_{s}}=-\kappa^{\ell}\left(\alpha_{3(j+1+m)}\right) \kappa^{\ell}\left(\gamma_{j+m}\right) f_{2}\left((j)_{s}, s-1\right) \kappa_{0} w_{4(j+m)+3 \rightarrow 4(j+m+s+1)+1} \otimes w_{4 j \rightarrow 4(j+s)+1} ; \\
b_{s+(j+1)_{s}, 3 s-1+(2)_{s}}=f_{2}\left((j)_{s},(s-2) s\right) \kappa^{\ell}\left(\gamma_{j+1+m}\right) \kappa_{0} w_{4(j+m+1)+2 \rightarrow 4(j+m+2)} \otimes w_{4 j+1 \rightarrow 4(j+1)} .
\end{gathered}
$$

(5) If $r_{0}=4$, then $\Omega^{4}\left(Y_{t}^{(5)}\right)$ is described with $(9 s \times 9 s)$-matrix with the following nonzero elements:

$$
\begin{gathered}
b_{j+3 s,(1)_{s}}=\kappa^{\ell}\left(\gamma_{j+m}\right) \kappa_{0} w_{4(j+m)+1 \rightarrow 4(j+m)} \otimes w_{4 j \rightarrow 4(j+1)+1}, \quad s=1 ; \\
b_{j+7 s,(1)_{s}}=-\kappa^{\ell}\left(\gamma_{j+m}\right) \kappa_{0} w_{4(j+m)+2 \rightarrow 4(j+m)} \otimes w_{4 j \rightarrow 4(j+1)+2}, \quad s=1 ; \\
b_{(j+1)_{s},(1)_{s}}=-f_{2}\left((j)_{s}, s-2\right) \kappa^{\ell}\left(\gamma_{j+m}\right) \kappa_{0} w_{4(j+m)+3 \rightarrow 4(j+m+1)} \otimes w_{4 j \rightarrow 4(j+1)}, \quad s>1 ; \\
b_{s+(j+1)_{s},(1)_{s}}=f_{2}\left((j)_{s}, s-2\right) \kappa^{\ell}\left(\gamma_{j+m}\right) \kappa_{0} e_{4(j+m+1)} \otimes w_{4 j \rightarrow 4(j+1)}, \quad s>1 ; \\
b_{(j+1)_{s}, 4 s+1-3 f(s, 1)}=f_{2}(s, 1) f_{1}\left((j)_{s}, s-2\right) \kappa^{\ell}\left(\gamma_{j+m}\right) \kappa^{\ell}\left(\alpha_{3(j+1+m)}\right) \kappa_{0} \\
w_{4(j+m)+3 \rightarrow 4(j+m+s+1+s f(s, 1))+2} \otimes w_{4(j+s+s f(s, 1))+1 \rightarrow 4(j+1)} ;
\end{gathered}
$$




$$
\begin{aligned}
b_{3 s+(j+1)_{s}, 7 s+(1)_{s}=}=-f_{2}\left((j)_{s},(s-2)_{s}\right) & \kappa^{\ell}\left(\gamma_{j+m}\right) \kappa_{0} \\
& w_{4(j+m+s+1+s f(s, 1))+1 \rightarrow 4(j+m+1)+3} \otimes w_{4 j+3 \rightarrow 4(j+1+s f(s, 1))+1} ; \\
b_{7 s+(j+1)_{s}, 7 s+(1)_{s}}=f_{2}\left((j)_{s},(s-2)_{s}\right) & \kappa^{\ell}\left(\gamma_{j+m}\right) \kappa_{0} \\
& w_{4(j+m+s+1+s f(s, 1))+2 \rightarrow 4(j+m+1)+3} \otimes w_{4 j+3 \rightarrow 4(j+1+s f(s, 1))+2 .}
\end{aligned}
$$

(6) If $r_{0}=5$ and $s=1$, then $\Omega^{5}\left(Y_{t}^{(5)}\right)$ is described with $(8 s \times 8 s)$-matrix with the following nonzero elements:

$$
\begin{gathered}
b_{j+s, s}=\kappa^{\ell}\left(\alpha_{3(j+m)}\right) \kappa_{0} w_{4(j+m) \rightarrow 4(j+m)+2} \otimes w_{4 j \rightarrow 4(j+1)+1} ; \\
b_{j+2 s, s}=\kappa^{\ell}\left(\alpha_{3(j+m)}\right) \kappa_{0} w_{4(j+m) \rightarrow 4(j+m)+2} \otimes w_{4 j \rightarrow 4 j+1} ; \\
b_{j-2 s, 2 s}=\kappa_{0} w_{4(j+m)+1 \rightarrow 4(j+m)+3} \otimes w_{4 j+1 \rightarrow 4 j} ; \\
b_{j-s, 2 s}=\kappa_{0} w_{4(j+m+1)+1 \rightarrow 4(j+m)+3} \otimes w_{4 j+1 \rightarrow 4 j} ; \\
b_{j-3 s, 6 s}=-2 \kappa^{\ell}\left(\alpha_{3(j+m)}\right) \kappa_{0} w_{4(j+m) \rightarrow 4(j+m+1)+1} \otimes w_{4 j+3 \rightarrow 4(j+1)+1} .
\end{gathered}
$$

(7) If $r_{0}=5$ and $s>1$, then $\Omega^{5}\left(Y_{t}^{(5)}\right)$ is described with $(8 s \times 8 s)$-matrix with the following nonzero elements:

$$
\begin{gathered}
b_{s+(j+1)_{s}, 1}=-\kappa^{\ell}\left(\alpha_{3(j+m)}\right) f_{1}\left((j)_{s}, s-2\right) \kappa_{0} w_{4(j+m+s+1)+1 \rightarrow 4(j+m+s+1)+2} \otimes w_{4 j \rightarrow 4(j+1)} \\
b_{(j+1)_{s}, s+1}=\kappa^{\ell}\left(\alpha_{3(j+m)}\right) f_{1}\left((j)_{s}, s-2\right) \kappa_{0} w_{4(j+m+s+1)+1 \rightarrow 4(j+m+s+1)+2} \otimes w_{4 j \rightarrow 4(j+1)} ; \\
b_{(j+1)_{s}, 3 s+1}=\kappa_{1} \kappa_{0} w_{4(j+m+s+1)+1 \rightarrow 4(j+m+1)+3} \otimes w_{4 j+1 \rightarrow 4(j+1)} ; \\
b_{s+(j+1)_{s}, 3 s+1}=\kappa_{1} \kappa_{0} w_{4(j+m+1)+1 \rightarrow 4(j+m+1)+3} \otimes w_{4 j+1 \rightarrow 4(j+1)} ; \\
b_{3 s+(j+1)_{s}, 5 s+1}=\kappa_{2} \kappa_{0} e_{4(j+m+2)} \otimes w_{4 j+2 \rightarrow 4(j+1)+1},
\end{gathered}
$$

where $\kappa_{1}=f_{2}(1, s-2) \kappa^{\ell}\left(\gamma_{3 s+1+m}\right), \kappa_{2}=\kappa^{\ell}\left(\gamma_{5 s+1+m}\right) \kappa^{\ell}\left(\gamma_{5 s+2+m}\right) f_{2}(1, s-2)$.

(8) If $r_{0}=6$ and $s=1$, then $\Omega^{6}\left(Y_{t}^{(5)}\right)$ is described with $(6 s \times 9 s)$-matrix with the following nonzero elements:

$$
\begin{gathered}
b_{j+2 s, 0}=-\kappa_{0} w_{4(j+m+1)+2 \rightarrow 4(j+m)+3} \otimes w_{4 j \rightarrow 4 j+1} ; \\
b_{j+3 s, 0}=-\kappa_{0} w_{4(j+m+1)+1 \rightarrow 4(j+m)+3} \otimes w_{4 j \rightarrow 4(j+1)+1} ; \\
b_{j+5 s, 0}=-\kappa_{0} w_{4(j+m)+2 \rightarrow 4(j+m)+3} \otimes w_{4 j \rightarrow 4 j+2} ; \\
b_{j+7 s, 0}=\kappa_{0} e_{4(j+m)+3} \otimes w_{4 j \rightarrow 4 j+3} ; \\
b_{j-s, s}=-\kappa^{\ell}\left(\alpha_{3(j+m)}\right) \kappa_{0} w_{4(j+m) \rightarrow 4(j+m+1)+2} \otimes w_{4(j+1)+1 \rightarrow 4 j} ; \\
b_{j-4 s, 5 s}=\kappa_{0} w_{4(j+m+1)+1 \rightarrow 4(j+m)} \otimes w_{4 j+3 \rightarrow 4(j+1)+1} ; \\
b_{j-2 s, 5 s}=-\kappa_{0} w_{4(j+m)+1 \rightarrow 4(j+m)} \otimes w_{4 j+3 \rightarrow 4 j+1} .
\end{gathered}
$$

(9) If $r_{0}=6$ and $s>1$, then $\Omega^{6}\left(Y_{t}^{(5)}\right)$ is described with $(6 s \times 9 s)$-matrix with the following two nonzero elements:

$$
\begin{aligned}
& b_{(j+1)_{s}, 2 s+1}=f_{1}\left((j)_{s}, s-2\right) \kappa^{\ell}\left(\alpha_{3(j+2+m)}\right) \kappa_{0} w_{4(j+m+1) \rightarrow 4(j+m+1)+2} \otimes w_{4(j+s)+1 \rightarrow 4(j+1)} ; \\
& b_{3 s+(j+1)_{s}, 4 s+1}=-f_{2}\left((j)_{s}, s-2\right) \kappa^{\ell}\left(\alpha_{3(j+2+m)}\right) \kappa_{0} e_{4(j+m+s+1)+1} \otimes w_{4(j+s)+2 \rightarrow 4(j+s+1)+1} .
\end{aligned}
$$

(10) If $r_{0}=7$ and $s=1$, then $\Omega^{7}\left(Y_{t}^{(5)}\right)$ is described with $(7 s \times 8 s)$-matrix with the following nonzero elements:

$$
\begin{gathered}
b_{j+4 s, 0}=-\kappa_{0} w_{4(j+m) \rightarrow 4(j+m)+3} \otimes w_{4 j \rightarrow 4 j+2} ; \\
b_{j+5 s, 0}=-\kappa_{0} w_{4(j+m) \rightarrow 4(j+m)+3} \otimes w_{4 j \rightarrow 4(j+1)+2} ; \\
b_{j, s}=\kappa_{0} w_{4(j+m)+2 \rightarrow 4(j+m)} \otimes w_{4(j+1)+1 \rightarrow 4 j} ;
\end{gathered}
$$




$$
\begin{gathered}
b_{j-2 s, 2 s}=-\kappa_{0} w_{4(j+m)+2 \rightarrow 4(j+m)} \otimes w_{4(j+1)+1 \rightarrow 4 j} ; \\
b_{j-s, 2 s}=\kappa_{0} w_{4(j+m+1)+2 \rightarrow 4(j+m)} \otimes w_{4(j+1)+1 \rightarrow 4 j} ; \\
b_{j-2 s, 3 s}=-\kappa^{\ell}\left(\alpha_{3(j+2+m)}\right) \kappa_{0} w_{4(j+m)+2 \rightarrow 4(j+m+1)+1} \otimes w_{4(j+1)+2 \rightarrow 4 j} ; \\
b_{j+2 s, 3 s}=\kappa^{\ell}\left(\alpha_{3(j+2+m)}\right) \kappa_{0} w_{4(j+m) \rightarrow 4(j+m+1)+1} \otimes w_{4(j+1)+2 \rightarrow 4 j+2} ; \\
b_{j-4 s, 4 s}=\kappa^{\ell}\left(\alpha_{3(j+2+m)}\right) \kappa_{0} w_{4(j+m)+2 \rightarrow 4(j+m+1)+1} \otimes w_{4(j+1)+2 \rightarrow 4 j} ; \\
b_{j-3 s, 5 s}=\kappa^{\ell}\left(\alpha_{3(j+2+m)}\right) \kappa_{0} w_{4(j+m)+3 \rightarrow 4(j+m)+2} \otimes w_{4 j+3 \rightarrow 4(j+1)+1} .
\end{gathered}
$$

(11) If $r_{0}=7$ and $s>1$, then $\Omega^{7}\left(Y_{t}^{(5)}\right)$ is described with $(7 s \times 8 s)$-matrix with the following nonzero elements:

$$
\begin{gathered}
b_{(j+1)_{s}, 1}=-\kappa^{\ell}\left(\gamma_{j+1+m}\right) f_{1}\left((j)_{s}, s-2\right) \kappa_{0} w_{4(j+m+1)+2 \rightarrow 4(j+m+1)+3} \otimes w_{4 j \rightarrow 4(j+1)} ; \\
b_{s+(j+1)_{s}, 2 s+1}=f_{2}\left((j)_{s}, s-2\right) \kappa_{0} w_{4(j+m+s+1)+2 \rightarrow 4(j+m+2)} \otimes w_{4(j+s)+1 \rightarrow 4(j+1)} ; \\
b_{7 s+(j)_{s}, 4 s+1}=-f_{1}\left((j)_{s}, s-2\right) \kappa^{\ell}\left(\alpha_{3(j+2+m)}\right) \kappa_{0} w_{4(j+m+s+1)+1 \rightarrow 4(j+m+s+2)+1} \otimes w_{4(j+s)+2 \rightarrow 4 j+3} .
\end{gathered}
$$

(12) If $r_{0}=8$ and $s=1$, then $\Omega^{8}\left(Y_{t}^{(5)}\right)$ is described with $(6 s \times 6 s)$-matrix with the following nonzero elements:

$$
\begin{gathered}
b_{j+2 s, 0}=-\kappa_{0} w_{4(j+m+1)+2 \rightarrow 4(j+m)} \otimes w_{4 j \rightarrow 4(j+1)+1} ; \\
b_{j+3 s, 0}=-\kappa_{0} w_{4(j+m+1)+1 \rightarrow 4(j+m)} \otimes w_{4 j \rightarrow 4 j+2} ; \\
b_{j-3 s, 3 s}=-\kappa^{\ell}\left(\alpha_{3(j+1+m)}\right) \kappa_{0} w_{4(j+m)+3 \rightarrow 4(j+m)+2} \otimes w_{4(j+1)+2 \rightarrow 4 j} ; \\
b_{j-s, 3 s}=-\kappa^{\ell}\left(\alpha_{3(j+1+m)}\right) \kappa_{0} e_{4(j+m)+2} \otimes w_{4(j+1)+2 \rightarrow 4 j+1} ; \\
b_{j-4 s, 4 s}=-\kappa^{\ell}\left(\alpha_{3(j+1+m)}\right) \kappa_{0} w_{4(j+m)+3 \rightarrow 4(j+m)+2} \otimes w_{4(j+1)+2 \rightarrow 4 j} ; \\
b_{j-4 s, 5 s}=\kappa_{0} w_{4(j+m+1)+2 \rightarrow 4(j+m)+3} \otimes w_{4 j+3 \rightarrow 4(j+1)+1} ; \\
b_{j-s, 5 s}=\kappa_{0} w_{4(j+m+1)+1 \rightarrow 4(j+m)+3} \otimes w_{4 j+3 \rightarrow 4 j+2} .
\end{gathered}
$$

(13) If $r_{0}=8$ and $s>1$, then $\Omega^{8}\left(Y_{t}^{(5)}\right)$ is described with $(6 s \times 6 s)$-matrix with the following two nonzero elements:

$$
\begin{gathered}
b_{(j+1)_{s}, 1}=-f_{1}(1, s-2) \kappa_{0} w_{4(j+m)+3 \rightarrow 4(j+m+1)} \otimes w_{4 j \rightarrow 4(j+1)} ; \\
b_{j, 2 s+1}=f_{1}\left((j)_{s}, s-2\right) \kappa^{\ell}\left(\alpha_{3(j+1+m)}\right) \kappa_{0} w_{4(j+m+s)+2 \rightarrow 4(j+m+s+1)+1} \otimes e_{4(j+s)+1} .
\end{gathered}
$$

(14) If $r_{0}=9$ and $s=1$, then $\Omega^{9}\left(Y_{t}^{(5)}\right)$ is described with $(6 s \times 7 s)$-matrix with the following two nonzero elements:

$$
\begin{gathered}
b_{j-s, 2 s}=\kappa^{\ell}\left(\alpha_{3(j+m)}\right) \kappa_{0} w_{4(j+m) \rightarrow 4(j+m+1)+1} \otimes w_{4(j+1)+1 \rightarrow 4 j+1} ; \\
b_{j+2 s, 4 s}=\kappa^{\ell}\left(\alpha_{3(j+m)}\right) \kappa_{0} w_{4(j+m)+2 \rightarrow 4(j+m+1)+2} \otimes w_{4(j+1)+2 \rightarrow 4 j+3} .
\end{gathered}
$$

(15) If $r_{0}=9$ and $s>1$, then $\Omega^{9}\left(Y_{t}^{(5)}\right)$ is described with $(6 s \times 7 s)$-matrix with the following two nonzero elements:

$$
\begin{aligned}
& b_{(j+1)_{s}, 2 s}=\kappa_{1} \kappa_{0} w_{4(j+m+1)+3 \rightarrow 4(j+m+s+2)+1} \otimes w_{4(j+s)+1 \rightarrow 4(j+1)} ; \\
& b_{(j+1)_{s}, 4 s}=\kappa_{1} \kappa_{0} w_{4(j+m+1)+3 \rightarrow 4(j+m+s+2)+2} \otimes w_{4(j+s)+2 \rightarrow 4(j+1)},
\end{aligned}
$$

where $\kappa_{1}=f_{2}(s, 3) \kappa^{\ell}\left(\alpha_{3(m+2)}\right)$.

(16) If $r_{0}=10$ and $s=1$, then $\Omega^{10}\left(Y_{t}^{(5)}\right)$ is described with $(7 s \times 6 s)$-matrix with the following two nonzero elements:

$$
\begin{gathered}
b_{j-3 s, 3 s}=-\kappa^{\ell}\left(\alpha_{3(j+1+m)}\right) \kappa_{0} w_{4(j+m) \rightarrow 4(j+m+1)+2} \otimes w_{4 j+1 \rightarrow 4 j} ; \\
b_{j+2 s, 3 s}=-\kappa^{\ell}\left(\alpha_{3(j+1+m)}\right) \kappa_{0} w_{4(j+m)+3 \rightarrow 4(j+m+1)+2} \otimes w_{4 j+1 \rightarrow 4 j+3} .
\end{gathered}
$$


(17) If $r_{0}=10$ and $s>1$, then $\Omega^{10}\left(Y_{t}^{(5)}\right)$ is described with $(7 s \times 6 s)$-matrix with the following two nonzero elements:

$$
\begin{gathered}
b_{(j+1)_{s}, s}=-f_{2}\left((j)_{s}, s-1\right) f_{2}\left((j)_{s}, s-3\right) \kappa^{\ell}\left(\alpha_{3(j+1+m)}\right) \kappa_{0} w_{4(j+m+1) \rightarrow 4(j+m+1)+1} \otimes w_{4 j \rightarrow 4(j+1)} \\
b_{5 s+(j)_{s}, 5 s}=f_{2}\left((j)_{s}, s-3\right) \kappa_{0} w_{4(j+m)+3 \rightarrow 4(j+m+1)+3} \otimes w_{4 j+2 \rightarrow 4 j+3} .
\end{gathered}
$$

(II) Represent an arbitrary $t_{0} \in \mathbb{N}$ in the form $t_{0}=11 \ell_{0}+r_{0}$, where $0 \leqslant r_{0} \leqslant 10$. Then $\Omega^{t_{0}}\left(Y_{t}^{(5)}\right)$ is a $\Omega^{r_{0}}\left(Y_{t}^{(5)}\right)$, whose left components twisted by $\sigma^{\ell_{0}}$, and coefficients multiplied by $(-1)^{\ell_{0}}$.

Proposition 22 (Translates for the case 6). (I) Let $r_{0} \in \mathbb{N}, r_{0}<11$. $r_{0}$-translates of the elements $Y_{t}^{(6)}$ are described by the following way.

(1) If $r_{0}=0$, then $\Omega^{0}\left(Y_{t}^{(6)}\right)$ is described with $(8 s \times 6 s)$-matrix with the following elements $b_{i j}$ : If $0 \leqslant j<s$, then

$$
b_{i j}=\left\{\begin{array}{l}
w_{4 j \rightarrow 4 j+2} \otimes e_{4 j}, \quad i=j \\
0, \quad \text { otherwise. }
\end{array}\right.
$$

If $s \leqslant j<3 s$, then $b_{i j}=0$.

If $3 s \leqslant j<4 s$, then

$$
b_{i j}=\left\{\begin{array}{l}
-\kappa^{\ell}\left(\alpha_{3(j+m+1)}\right) w_{4 j+1 \rightarrow 4 j+3} \otimes e_{4 j+1}, \quad i=j-s \\
0, \quad \text { otherwise }
\end{array}\right.
$$

If $4 s \leqslant j<6 s$, then

$$
b_{i j}=\left\{\begin{array}{l}
-\kappa^{\ell}\left(\alpha_{3(j+m+2)}\right) w_{4 j+2 \rightarrow 4(j+1)} \otimes e_{4 j+2}, \quad i=j-s, j<5 s-1 \text { or } j=6 s-1 \\
0, \quad \text { otherwise. }
\end{array}\right.
$$

If $6 s \leqslant j<8 s$, then

$$
b_{i j}=\left\{\begin{array}{l}
w_{4 j+3 \rightarrow 4(j+1)+1} \otimes e_{4 j+3}, \quad i=5 s+(j)_{s}, j<7 s-1 \text { or } j=8 s-1 \\
0, \quad \text { otherwise. }
\end{array}\right.
$$

(2) If $r_{0}=1$, then $\Omega^{1}\left(Y_{t}^{(6)}\right)$ is described with $(9 s \times 7 s)$-matrix with the following elements $b_{i j}$ : If $0 \leqslant j<s$, then

$$
b_{i j}=\left\{\begin{array}{l}
-\kappa^{\ell}\left(\alpha_{3(j+m+1)}\right) w_{4(j+m+s)+1 \rightarrow 4(j+m)+3} \otimes e_{4 j}, \quad i=j+s \\
0, \quad \text { otherwise. }
\end{array}\right.
$$

If $s \leqslant j<2 s$, then

$$
b_{i j}=\left\{\begin{array}{l}
-\kappa^{\ell}\left(\alpha_{3(j+m+2)}\right) w_{4(j+m+s)+1 \rightarrow 4(j+m+1)} \otimes e_{4 j}, \quad i=j-s \\
-\kappa^{\ell}\left(\alpha_{3(j+m+2)}\right) w_{4(j+m+s)+2 \rightarrow 4(j+m+1)} \otimes w_{4 j \rightarrow 4(j+s)+1}, \quad i=j+s \\
0, \quad \text { otherwise. }
\end{array}\right.
$$

If $2 s \leqslant j<2 s-1$, then $b_{i j}=0$. 
If $2 s-1 \leqslant j<2 s$, then

$$
b_{i j}=\left\{\begin{array}{l}
-\kappa^{\ell}\left(\alpha_{3(j+m+2)}\right) w_{4(j+m)+3 \rightarrow 4(j+m+1)} \otimes w_{4 j \rightarrow 4(j+s)+2}, \quad i=j+3 s \\
\kappa^{\ell}\left(\alpha_{3(j+m+2)}\right) w_{4(j+m)+3 \rightarrow 4(j+m+1)} \otimes w_{4 j \rightarrow 4 j+2}, \quad i=j+4 s \\
0, \quad \text { otherwise. }
\end{array}\right.
$$

If $2 s \leqslant j<3 s-1$, then $b_{i j}=0$.

If $3 s-1 \leqslant j<3 s$, then

$$
b_{i j}=\left\{\begin{array}{l}
-w_{4(j+m)+3 \rightarrow 4(j+m+s+1)+1} \otimes w_{4 j+1 \rightarrow 4 j+2}, \quad i=j+2 s \\
0, \quad \text { otherwise. }
\end{array}\right.
$$

If $3 s \leqslant j<4 s-1$, then

$$
b_{i j}=\left\{\begin{array}{l}
-w_{4(j+m)+3 \rightarrow 4(j+m+s+1)+1} \otimes w_{4 j+1 \rightarrow 4 j+2}, \quad i=j+2 s \\
0, \quad \text { otherwise. }
\end{array}\right.
$$

If $4 s-1 \leqslant j<4 s$, then $b_{i j}=0$.

If $4 s \leqslant j<5 s-1$, then

$$
b_{i j}=\left\{\begin{array}{l}
-w_{4(j+m)+3 \rightarrow 4(j+m+1)+1} \otimes e_{4 j+2}, \quad i=j ; \\
0, \quad \text { otherwise. }
\end{array}\right.
$$

If $5 s-1 \leqslant j<6 s-1$, then $b_{i j}=0$.

If $6 s-1 \leqslant j<6 s$, then

$$
b_{i j}=\left\{\begin{array}{l}
w_{4(j+m+1)+1 \rightarrow 4(j+m+1)+2} \otimes w_{4(j+s)+2 \rightarrow 4(j+1)}, \quad i=(j+1)_{s} \\
w_{4(j+m)+3 \rightarrow 4(j+m+1)+2} \otimes e_{4(j+s)+2}, \quad i=j-s \\
0, \quad \text { otherwise. }
\end{array}\right.
$$

If $6 s \leqslant j<7 s-1$, then $b_{i j}=0$.

If $7 s-1 \leqslant j<7 s$, then

$$
b_{i j}=\left\{\begin{array}{l}
-w_{4(j+m)+3 \rightarrow 4(j+m+s+1)+1} \otimes e_{4(j+s)+2}, \quad i=j-s \\
0, \quad \text { otherwise. }
\end{array}\right.
$$

If $7 s \leqslant j<8 s-1$, then

$$
b_{i j}=\left\{\begin{array}{l}
w_{4(j+m+s+1)+1 \rightarrow 4(j+m+s+1)+2} \otimes w_{4 j+2 \rightarrow 4(j+1)}, \quad i=(j+1)_{s} \\
w_{4(j+m)+3 \rightarrow 4(j+m+s+1)+2} \otimes e_{4 j+2}, \quad i=j-2 s \\
0, \quad \text { otherwise. }
\end{array}\right.
$$

If $8 s-1 \leqslant j<8 s$, then $b_{i j}=0$.

If $8 s \leqslant j<9 s$, then

$$
b_{i j}=\left\{\begin{array}{l}
\kappa^{\ell}\left(\alpha_{3(j+m+2)}\right) w_{4(j+m+1) \rightarrow 4(j+m+1)+3} \otimes e_{4 j+3}, \quad i=j-2 s \\
0, \quad \text { otherwise. }
\end{array}\right.
$$


(3) If $r_{0}=2$, then $\Omega^{2}\left(Y_{t}^{(6)}\right)$ is described with $(8 s \times 6 s)$-matrix with the following elements $b_{i j}$ : If $0 \leqslant j<s$, then

If $s \leqslant j<2 s$, then $b_{i j}=0$.

$$
b_{i j}=\left\{\begin{array}{l}
-w_{4(j+m)-1 \rightarrow 4(j+m)+1} \otimes e_{4 j}, \quad i=j \\
0, \quad \text { otherwise. }
\end{array}\right.
$$

If $2 s \leqslant j<4 s$, then

$$
b_{i j}=\left\{\begin{array}{c}
-\kappa^{\ell}\left(\alpha_{3(j+m+2)}\right) w_{4(j+m)+3 \rightarrow 4(j+m+1)} \otimes w_{4 j+1 \rightarrow 4(j+1)}, \\
i=(j+1)_{s}, j<3 s-1 \text { or } j=4 s-1 \\
0, \quad \text { otherwise. }
\end{array}\right.
$$

If $4 s \leqslant j<5 s$, then

$$
b_{i j}=\left\{\begin{array}{l}
-\kappa^{\ell}\left(\alpha_{3(j+m+1)}\right) w_{4(j+m+s)+1 \rightarrow 4(j+m)+3} \otimes e_{4 j+2}, \quad i=j-s ; \\
0, \quad \text { otherwise. }
\end{array}\right.
$$

If $5 s \leqslant j<6 s$, then

$$
b_{i j}=\left\{\begin{array}{l}
-\kappa^{\ell}\left(\alpha_{3(j+m+1)}\right) w_{4(j+m+s)+1 \rightarrow 4(j+m)+3} \otimes e_{4 j+2}, \quad i=j-s \\
0, \quad \text { otherwise. }
\end{array}\right.
$$

If $6 s \leqslant j<8 s$, then $b_{i j}=0$.

(4) If $r_{0}=3$, then $\Omega^{3}\left(Y_{t}^{(6)}\right)$ is described with $(9 s \times 8 s)$-matrix with the following elements $b_{i j}$ : If $0 \leqslant j<s$, then

$$
b_{i j}=\left\{\begin{array}{l}
\kappa^{\ell}\left(\alpha_{3(j+m+2)}\right) w_{4(j+m)+2 \rightarrow 4(j+m+1)} \otimes e_{4 j}, \quad i=j ; \\
\kappa^{\ell}\left(\alpha_{3(j+m+2)}\right) w_{4(j+m)+3 \rightarrow 4(j+m+1)} \otimes w_{4 j \rightarrow 4 j+1}, \quad i=j+2 s \\
0, \quad \text { otherwise. }
\end{array}\right.
$$

If $s \leqslant j<3 s-1$, then $b_{i j}=0$.

If $3 s-1 \leqslant j<3 s$, then

$$
b_{i j}=\left\{\begin{array}{l}
e_{4(j+m+s+1)+2} \otimes w_{4 j+1 \rightarrow 4(j+1)}, \quad i=(j+1)_{s} \\
0, \quad \text { otherwise. }
\end{array}\right.
$$

If $3 s \leqslant j<2 s$, then $b_{i j}=0$.

If $2 s \leqslant j<3 s-1$, then

$$
b_{i j}=\left\{\begin{array}{l}
-e_{4(j+m+s+1)+2} \otimes w_{4 j+1 \rightarrow 4(j+1)}, \quad i=s+(j+1)_{s} ; \\
0, \quad \text { otherwise. }
\end{array}\right.
$$

If $3 s-1 \leqslant j<4 s$, then $b_{i j}=0$.

If $4 s \leqslant j<5 s-1$, then

$$
b_{i j}=\left\{\begin{array}{l}
e_{4(j+m+1)+2} \otimes w_{4(j+s)+1 \rightarrow 4(j+1)}, \quad i=(j+1)_{s} \\
0, \quad \text { otherwise. }
\end{array}\right.
$$


If $5 s-1 \leqslant j<5 s$, then

$$
b_{i j}=\left\{\begin{array}{l}
-e_{4(j+m+1)+2} \otimes w_{4(j+s)+1 \rightarrow 4(j+1)}, \quad i=s+(j+1)_{s} ; \\
0, \quad \text { otherwise. }
\end{array}\right.
$$

If $5 s \leqslant j<7 s$, then $b_{i j}=0$.

If $7 s \leqslant j<8 s$, then

$$
b_{i j}=\left\{\begin{array}{l}
\kappa^{\ell}\left(\alpha_{3(j+m+2)}\right) f_{1}(j, 8 s-1) w_{4(j+m+s-f(j, 8 s-1) s+1)+2 \rightarrow 4(j+m+1)+3} \otimes w_{4 j+3 \rightarrow 4(j+1)} \\
\quad i=(j+1)_{s} \\
\kappa^{\ell}\left(\alpha_{3(j+m+2)}\right) f_{1}(j, 8 s-1) e_{4(j+m+1)+3} \otimes w_{4 j+3 \rightarrow 4(j+s-f(j, 8 s-1) s+1)+1} \\
\quad i=2 s+(j+1)_{s} \\
0, \quad \text { otherwise. }
\end{array}\right.
$$

If $8 s \leqslant j<9 s$, then $b_{i j}=0$.

(5) If $r_{0}=4$, then $\Omega^{4}\left(Y_{t}^{(6)}\right)$ is described with $(8 s \times 9 s)$-matrix with the following elements $b_{i j}$ : If $0 \leqslant j<s$, then

$$
b_{i j}=\left\{\begin{array}{l}
-w_{4(j+m)-1 \rightarrow 4(j+m)+2} \otimes e_{4 j}, \quad i=j \\
w_{4(j+m)+1 \rightarrow 4(j+m)+2} \otimes w_{4 j \rightarrow 4(j+s)+1}, \quad i=j+3 s \\
-e_{4(j+m)+2} \otimes w_{4 j \rightarrow 4(j+s)+2}, \quad i=j+7 s \\
0, \quad \text { otherwise. }
\end{array}\right.
$$

If $s \leqslant j<2 s$, then

$$
b_{i j}=\left\{\begin{array}{l}
w_{4(j+m)+1 \rightarrow 4(j+m)+2} \otimes w_{4 j \rightarrow 4(j+s)+1}, \quad i=j+s \\
-e_{4(j+m)+2} \otimes w_{4 j \rightarrow 4(j+s)+2}, \quad i=j+4 s \\
0, \quad \text { otherwise. }
\end{array}\right.
$$

If $2 s \leqslant j<4 s$, then

$b_{i j}=\left\{\begin{array}{l}-\kappa^{\ell}\left(\alpha_{3(j+m+1)}\right) e_{4(j+m)+3} \otimes w_{4 j+1 \rightarrow 4(j+1)}, \quad i=(j+1)_{s}, j<3 s-1 \text { or } j=4 s-1 ; \\ 0, \quad \text { otherwise. }\end{array}\right.$

If $4 s \leqslant j<8 s$, then $b_{i j}=0$.

(6) If $r_{0}=5$, then $\Omega^{5}\left(Y_{t}^{(6)}\right)$ is described with $(6 s \times 8 s)$-matrix with the following elements $b_{i j}$ : If $0 \leqslant j<s$, then

$$
b_{i j}=\left\{\begin{array}{l}
\kappa^{\ell}\left(\alpha_{3(j+m+1)}\right) w_{4(j+m)+1 \rightarrow 4(j+m)+3} \otimes e_{4 j}, \quad i=j \\
\kappa^{\ell}\left(\alpha_{3(j+m+1)}\right) w_{4(j+m+s)+1 \rightarrow 4(j+m)+3} \otimes e_{4 j}, \quad i=j+s \\
-\kappa^{\ell}\left(\alpha_{3(j+m+1)}\right) e_{4(j+m)+3} \otimes w_{4 j \rightarrow 4 j+2}, \quad i=j+4 s \\
-\kappa^{\ell}\left(\alpha_{3(j+m+1)}\right) e_{4(j+m)+3} \otimes w_{4 j \rightarrow 4(j+s)+2}, \quad i=j+5 s \\
0, \quad \text { otherwise. }
\end{array}\right.
$$

If $s \leqslant j<5 s$, then $b_{i j}=0$. 
If $5 s \leqslant j<6 s$, then

$$
b_{i j}=\left\{\begin{array}{l}
\kappa^{\ell}\left(\alpha_{3(j+m+3)}\right) f_{1}(j, 6 s-1) e_{4(j+m+2)} \otimes w_{4 j+3 \rightarrow 4(j+s-f(j, 6 s-1) s+1)+1} \\
\quad i=2 s+(j+1)_{s} \\
0, \quad \text { otherwise. }
\end{array}\right.
$$

(7) If $r_{0}=6$, then $\Omega^{6}\left(Y_{t}^{(6)}\right)$ is described with $(7 s \times 9 s)$-matrix with the following elements $b_{i j}$ : If $0 \leqslant j<s$, then

$$
b_{i j}=\left\{\begin{array}{l}
-\kappa^{\ell}\left(\alpha_{3(j+m+1)}\right) w_{4(j+m) \rightarrow 4(j+m)+3} \otimes e_{4 j}, \quad i=j ; \\
\kappa^{\ell}\left(\alpha_{3(j+m+1)}\right) w_{4(j+m)+1 \rightarrow 4(j+m)+3} \otimes w_{4 j \rightarrow 4 j+1}, \quad i=j+s \\
\kappa^{\ell}\left(\alpha_{3(j+m+1)}\right) w_{4(j+m+s)+1 \rightarrow 4(j+m)+3} \otimes w_{4 j \rightarrow 4(j+s)+1}, \quad i=j+3 s \\
0, \quad \text { otherwise. }
\end{array}\right.
$$

If $s \leqslant j<3 s$, then

$$
b_{i j}=\left\{\begin{array}{l}
\kappa^{\ell}\left(\alpha_{3(j+m+2)}\right) e_{4(j+m+1)} \otimes w_{4(j+s)+1 \rightarrow 4(j+1)} \\
i=(j+1)_{s}, j<2 s-1 \text { or } j=3 s-1 \\
0, \quad \text { otherwise. }
\end{array}\right.
$$

If $3 s \leqslant j<5 s$, then

$$
b_{i j}=\left\{\begin{array}{l}
-w_{4(j+m+1) \rightarrow 4(j+m+1)+1} \otimes w_{4(j+s)+2 \rightarrow 4(j+1)} \\
i=(j+1)_{s}, j<4 s-1 \text { or } j=5 s-1 \\
0, \quad \text { otherwise. }
\end{array}\right.
$$

If $5 s \leqslant j<6 s$, then

$$
b_{i j}=\left\{\begin{array}{l}
f_{1}(j, 6 s-1) w_{4(j+m+1) \rightarrow 4(j+m+s+1)+2} \otimes w_{4 j+3 \rightarrow 4(j+1)}, \quad i=(j+1)_{s} \\
0, \quad \text { otherwise. }
\end{array}\right.
$$

If $6 s \leqslant j<6 s-1$, then $b_{i j}=0$.

If $6 s-1 \leqslant j<6 s$, then

$$
b_{i j}=\left\{\begin{array}{l}
w_{4(j+m+s+1)+1 \rightarrow 4(j+m+s+1)+2} \otimes w_{4 j+3 \rightarrow 4(j+s+1)+1}, \quad i=3 s+(j+1)_{s} \\
0, \quad \text { otherwise. }
\end{array}\right.
$$

If $6 s \leqslant j<5 s$, then $b_{i j}=0$.

If $5 s \leqslant j<6 s-1$, then

$$
b_{i j}= \begin{cases}-e_{4(j+m+s+1)+2} \otimes w_{4 j+3 \rightarrow 4(j+1)+1}, & i=4 s+(j+1)_{s} ; \\ e_{4(j+m+s+1)+2} \otimes w_{4 j+3 \rightarrow 4(j+s+1)+2}, & i=5 s+(j+1)_{s} ; \\ 0, \quad \text { otherwise. } & \end{cases}
$$

If $6 s-1 \leqslant j<6 s$, then $b_{i j}=0$. 
HOCHSCHILD COHOMOLOGY RING FOR SELF-INJECTIVE ALGEBRAS OF TREE CLASS $E_{6}$. II. 61 If $6 s \leqslant j<7 s$, then

$$
b_{i j}=\left\{\begin{array}{l}
-f_{1}(j, 7 s-1) w_{4(j+m+1) \rightarrow 4(j+m+s+1)+2} \otimes w_{4 j+3 \rightarrow 4(j+1)}, \quad i=(j+1)_{s} ; \\
0, \quad \text { otherwise. }
\end{array}\right.
$$

If $7 s \leqslant j<6 s$, then $b_{i j}=0$.

If $6 s \leqslant j<7 s-1$, then

$$
b_{i j}=\left\{\begin{array}{l}
w_{4(j+m+s+1)+1 \rightarrow 4(j+m+s+1)+2} \otimes w_{4 j+3 \rightarrow 4(j+s+1)+1}, \quad i=3 s+(j+1)_{s} ; \\
0, \quad \text { otherwise. }
\end{array}\right.
$$

If $7 s-1 \leqslant j<7 s$, then

$$
b_{i j}= \begin{cases}-e_{4(j+m+s+1)+2} \otimes w_{4 j+3 \rightarrow 4(j+1)+1}, & i=4 s+(j+1)_{s} ; \\ e_{4(j+m+s+1)+2} \otimes w_{4 j+3 \rightarrow 4(j+s+1)+2}, & i=5 s+(j+1)_{s} \\ 0, \quad \text { otherwise. } & \end{cases}
$$

(8) If $r_{0}=7$, then $\Omega^{7}\left(Y_{t}^{(6)}\right)$ is described with $(6 s \times 8 s)$-matrix with the following elements $b_{i j}$ : If $0 \leqslant j<s$, then

$$
b_{i j}=\left\{\begin{array}{l}
\kappa^{\ell}\left(\alpha_{3(j+m+2)}\right) w_{4(j+m+s)+2 \rightarrow 4(j+m+1)} \otimes e_{4 j}, \quad i=j+s \\
-\kappa^{\ell}\left(\alpha_{3(j+m+2)}\right) w_{4(j+m)+3 \rightarrow 4(j+m+1)} \otimes w_{4 j \rightarrow 4 j+1}, \quad i=j+2 s \\
-\kappa^{\ell}\left(\alpha_{3(j+m+2)}\right) e_{4(j+m+1)} \otimes w_{4 j \rightarrow 4 j+2}, \quad i=j+4 s \\
-\kappa^{\ell}\left(\alpha_{3(j+m+2)}\right) e_{4(j+m+1)} \otimes w_{4 j \rightarrow 4(j+s)+2}, \quad i=j+5 s \\
0, \quad \text { otherwise. }
\end{array}\right.
$$

If $s \leqslant j<4 s-1$, then $b_{i j}=0$.

If $4 s-1 \leqslant j<4 s$, then

$$
b_{i j}=\left\{\begin{array}{l}
e_{4(j+m+1)+2} \otimes w_{4(j+s)+2 \rightarrow 4(j+1)}, \quad i=(j+1)_{s} \\
0, \quad \text { otherwise. }
\end{array}\right.
$$

If $4 s \leqslant j<5 s-1$, then

$$
b_{i j}=\left\{\begin{array}{l}
e_{4(j+m+1)+2} \otimes w_{4(j+s)+2 \rightarrow 4(j+1)}, \quad i=(j+1)_{s} \\
0, \quad \text { otherwise. }
\end{array}\right.
$$

If $5 s-1 \leqslant j<5 s$, then $b_{i j}=0$.

If $5 s \leqslant j<6 s$, then

$$
b_{i j}=\left\{\begin{array}{l}
\kappa^{\ell}\left(\alpha_{3(j+m+2)}\right) w_{4(j+m+s-f(j, 6 s-1) s+1)+2 \rightarrow 4(j+m+1)+3} \otimes w_{4 j+3 \rightarrow 4(j+1)}, \quad i=(j+1)_{s} \\
0, \quad \text { otherwise. }
\end{array}\right.
$$

(9) If $r_{0}=8$, then $\Omega^{8}\left(Y_{t}^{(6)}\right)$ is described with $(6 s \times 6 s)$-matrix with the following elements $b_{i j}$ : 
If $0 \leqslant j<s$, then

$$
b_{i j}=\left\{\begin{array}{l}
\kappa^{\ell}\left(\alpha_{3(j+m+2)}\right) w_{4(j+m)+3 \rightarrow 4(j+m+1)} \otimes w_{4 j \rightarrow 4(j+1)}, \quad i=(j+1)_{s} \\
\kappa^{\ell}\left(\alpha_{3(j+m+2)}\right) w_{4(j+m)+2 \rightarrow 4(j+m+1)} \otimes w_{4 j \rightarrow 4 j+1}, \quad i=j+s \\
\kappa^{\ell}\left(\alpha_{3(j+m+2)}\right) w_{4(j+m+s)+2 \rightarrow 4(j+m+1)} \otimes w_{4 j \rightarrow 4(j+s)+1}, \quad i=j+2 s \\
\kappa^{\ell}\left(\alpha_{3(j+m+2)}\right) e_{4(j+m+1)} \otimes w_{4 j \rightarrow 4 j+3}, \quad i=j+5 s \\
0, \quad \text { otherwise. }
\end{array}\right.
$$

If $s \leqslant j<3 s$, then

$b_{i j}=\left\{\begin{array}{l}w_{4(j+m)+3 \rightarrow 4(j+m+1)+1} \otimes w_{4(j+s)+1 \rightarrow 4(j+1)}, \quad i=(j+1)_{s}, j<2 s-1 \text { or } j=3 s-1 \\ 0, \quad \text { otherwise. }\end{array}\right.$

If $3 s \leqslant j<4 s-1$, then

$$
b_{i j}=\left\{\begin{array}{l}
w_{4(j+m)+3 \rightarrow 4(j+m+1)+2} \otimes w_{4(j+s)+2 \rightarrow 4(j+1)}, \quad i=(j+1)_{s} \\
w_{4(j+m+1)+1 \rightarrow 4(j+m+1)+2} \otimes w_{4(j+s)+2 \rightarrow 4(j+s+1)+2}, \quad i=3 s+(j+1)_{s} \\
0, \quad \text { otherwise. }
\end{array}\right.
$$

If $4 s-1 \leqslant j<4 s$, then

$$
b_{i j}=\left\{\begin{array}{l}
w_{4(j+m+1)+1 \rightarrow 4(j+m+1)+2} \otimes w_{4(j+s)+2 \rightarrow 4(j+s+1)+2}, \quad i=4 s+(j+1)_{s} \\
0, \quad \text { otherwise. }
\end{array}\right.
$$

If $4 s \leqslant j<5 s-1$, then $b_{i j}=0$.

If $5 s-1 \leqslant j<5 s$, then

$$
b_{i j}=\left\{\begin{array}{l}
w_{4(j+m)+3 \rightarrow 4(j+m+1)+2} \otimes w_{4(j+s)+2 \rightarrow 4(j+1)}, \quad i=(j+1)_{s} \\
w_{4(j+m+1)+1 \rightarrow 4(j+m+1)+2} \otimes w_{4(j+s)+2 \rightarrow 4(j+s+1)+2}, \quad i=3 s+(j+1)_{s} \\
0, \quad \text { otherwise. }
\end{array}\right.
$$

If $5 s \leqslant j<4 s$, then $b_{i j}=0$.

If $4 s \leqslant j<5 s-1$, then

$$
b_{i j}=\left\{\begin{array}{l}
w_{4(j+m+1)+1 \rightarrow 4(j+m+1)+2} \otimes w_{4(j+s)+2 \rightarrow 4(j+s+1)+2}, \quad i=4 s+(j+1)_{s} \\
0, \quad \text { otherwise }
\end{array}\right.
$$

If $5 s-1 \leqslant j<5 s$, then $b_{i j}=0$. 
If $5 s \leqslant j<6 s$, then

$$
b_{i j}=\left\{\begin{array}{l}
-\kappa^{\ell}\left(\alpha_{3(j+m+2)}\right) w_{4(j+m)+3 \rightarrow 4(j+m+1)+3} \otimes w_{4 j+3 \rightarrow 4(j+1)}, \quad i=(j+1)_{s} ; \\
-\kappa^{\ell}\left(\alpha_{3(j+m+2)}\right) w_{4(j+m+1+f(j, 6 s-1) s)+2 \rightarrow 4(j+m+1)+3} \otimes w_{4 j+3 \rightarrow 4(j+1+f(j, 6 s-1) s)+1}, \\
\quad i=2 s+(j+1)_{s} ; \\
-\kappa^{\ell}\left(\alpha_{3(j+m+2)}\right) w_{4(j+m+1+f(j, 6 s-1) s)+1 \rightarrow 4(j+m+1)+3} \otimes w_{4 j+3 \rightarrow 4(j+s-f(j, 6 s-1) s+1)+2}, \\
\quad i=3 s+(j+1)_{s} ; \\
0, \quad \text { otherwise. }
\end{array}\right.
$$

(10) If $r_{0}=9$, then $\Omega^{9}\left(Y_{t}^{(6)}\right)$ is described with $(7 s \times 7 s)$-matrix with the following elements $b_{i j}$ : If $0 \leqslant j<s$, then

$$
b_{i j}=\left\{\begin{array}{l}
w_{4(j+m)+3 \rightarrow 4(j+m+s+1)+1} \otimes e_{4 j}, \quad i=j \\
w_{4(j+m+1) \rightarrow 4(j+m+s+1)+1} \otimes w_{4 j \rightarrow 4 j+1}, \quad i=j+s \\
0, \quad \text { otherwise. }
\end{array}\right.
$$

If $s \leqslant j<2 s$, then

$$
b_{i j}=\left\{\begin{array}{l}
w_{4(j+m+1) \rightarrow 4(j+m+s+1)+1} \otimes w_{4 j \rightarrow 4(j+s)+1,} \quad i=j \\
0, \quad \text { otherwise. }
\end{array}\right.
$$

If $2 s \leqslant j<5 s-1$, then $b_{i j}=0$.

If $5 s-1 \leqslant j<6 s-1$, then

$$
b_{i j}=\left\{\begin{array}{l}
-f_{2}(j, 5 s-1) \kappa^{\ell}\left(\alpha_{3(j+m+2)}\right) e_{4(j+m+1)+3} \otimes w_{4 j+2 \rightarrow 4(j+1)}, \quad i=(j+1)_{s} \\
0, \quad \text { otherwise. }
\end{array}\right.
$$

If $6 s-1 \leqslant j<6 s$, then $b_{i j}=0$.

If $6 s \leqslant j<7 s$, then

$b_{i j}=\left\{\begin{array}{l}f_{2}(j, 7 s-1) \kappa^{\ell}\left(\alpha_{3(j+m+3)}\right) e_{4(j+m+2)} \otimes w_{4 j+3 \rightarrow 4(j+1+f(j, 7 s-1) s)+1}, \quad i=s+(j+1)_{s} \\ 0, \quad \text { otherwise. }\end{array}\right.$ $b_{i j}:$

(11) If $r_{0}=10$, then $\Omega^{10}\left(Y_{t}^{(6)}\right)$ is described with $(6 s \times 6 s)$-matrix with the following elements 
If $0 \leqslant j<s$, then

$$
b_{i j}=\left\{\begin{array}{l}
\kappa^{\ell}\left(\alpha_{3(j+m+1)}\right) w_{4(j+m) \rightarrow 4(j+m)+3} \otimes e_{4 j}, \quad i=j ; \\
-\kappa^{\ell}\left(\alpha_{3(j+m+1)}\right) w_{4(j+m+s)+1 \rightarrow 4(j+m)+3} \otimes w_{4 j \rightarrow 4 j+1}, \quad i=j+s \\
\kappa^{\ell}\left(\alpha_{3(j+m+1)}\right) w_{4(j+m)+1 \rightarrow 4(j+m)+3} \otimes w_{4 j \rightarrow 4(j+s)+1}, \quad i=j+2 s \\
\kappa^{\ell}\left(\alpha_{3(j+m+1)}\right) w_{4(j+m+s)+2 \rightarrow 4(j+m)+3} \otimes w_{4 j \rightarrow 4 j+2}, \quad i=j+3 s \\
-\kappa^{\ell}\left(\alpha_{3(j+m+1)}\right) w_{4(j+m)+2 \rightarrow 4(j+m)+3} \otimes w_{4 j \rightarrow 4(j+s)+2}, \quad i=j+4 s \\
\kappa^{\ell}\left(\alpha_{3(j+m+1)}\right) e_{4(j+m)+3} \otimes w_{4 j \rightarrow 4 j+3}, \quad i=j+5 s \\
0, \quad \text { otherwise. }
\end{array}\right.
$$

If $s \leqslant j<2 s$, then

$$
b_{i j}=\left\{\begin{array}{l}
f_{1}(j, 2 s-1) w_{4(j+m+1) \rightarrow 4(j+m+1)+2} \otimes w_{4(j+s)+1 \rightarrow 4(j+1)}, \quad i=(j+1)_{s} \\
0, \quad \text { otherwise. }
\end{array}\right.
$$

If $2 s \leqslant j<s$, then $b_{i j}=0$.

If $s \leqslant j<2 s-1$, then

$$
b_{i j}=\left\{\begin{array}{l}
-w_{4(j+m+1)+1 \rightarrow 4(j+m+1)+2} \otimes w_{4(j+s)+1 \rightarrow 4(j+s+1)+1}, \quad i=s+(j+1)_{s} \\
0, \quad \text { otherwise. }
\end{array}\right.
$$

If $2 s-1 \leqslant j<2 s$, then

$$
b_{i j}=\left\{\begin{array}{l}
-w_{4(j+m+1)+1 \rightarrow 4(j+m+1)+2} \otimes w_{4(j+s)+1 \rightarrow 4(j+s+1)+1}, \quad i=2 s+(j+1)_{s} \\
0, \quad \text { otherwise. }
\end{array}\right.
$$

If $2 s \leqslant j<3 s$, then

$$
b_{i j}=\left\{\begin{array}{l}
w_{4(j+m)+2 \rightarrow 4(j+m+1)+2} \otimes w_{4(j+s)+1 \rightarrow 4(j+s)+2,} \quad i=j+2 s \\
-w_{4(j+m)+3 \rightarrow 4(j+m+1)+2} \otimes w_{4(j+s)+1 \rightarrow 4 j+3}, \quad i=j+3 s \\
0, \quad \text { otherwise. }
\end{array}\right.
$$

If $3 s \leqslant j<6 s$, then $b_{i j}=0$.

(II) Represent an arbitrary $t_{0} \in \mathbb{N}$ in the form $t_{0}=11 \ell_{0}+r_{0}$, where $0 \leqslant r_{0} \leqslant 10$. Then $\Omega^{t_{0}}\left(Y_{t}^{(6)}\right)$ is a $\Omega^{r_{0}}\left(Y_{t}^{(6)}\right)$, whose left components twisted by $\sigma^{\ell_{0}}$.

Proposition 23 (Translates for the case 7). (I) Let $r_{0} \in \mathbb{N}, r_{0}<11$. $r_{0}$-translates of the elements $Y_{t}^{(7)}$ are described by the following way.

(1) If $r_{0}=0$, then $\Omega^{0}\left(Y_{t}^{(7)}\right)$ is described with $(8 s \times 6 s)$-matrix with the following elements $b_{i j}$ : If $0 \leqslant j<2 s$, then

$$
b_{i j}=\left\{\begin{array}{l}
w_{4 j \rightarrow 4(j+s)+2} \otimes e_{4 j}, \quad i=(j)_{s} \\
0, \quad \text { otherwise }
\end{array}\right.
$$


If $2 s \leqslant j<4 s$, then

$$
b_{i j}=\left\{\begin{array}{l}
w_{4 j+1 \rightarrow 4 j+3} \otimes e_{4 j+1}, \quad i=j-s \\
0, \quad \text { otherwise }
\end{array}\right.
$$

If $4 s \leqslant j<6 s$, then

$$
b_{i j}=\left\{\begin{array}{l}
w_{4 j+2 \rightarrow 4(j+1)} \otimes e_{4 j+2}, \quad i=j-s \\
0, \quad \text { otherwise. }
\end{array}\right.
$$

If $6 s \leqslant j<8 s$, then $b_{i j}=0$.

(2) If $r_{0}=1$, then $\Omega^{1}\left(Y_{t}^{(7)}\right)$ is described with $(9 s \times 7 s)$-matrix with the following elements $b_{i j}$ : If $0 \leqslant j<s$, then

$$
b_{i j}=\left\{\begin{array}{l}
w_{4(j+m)+1 \rightarrow 4(j+m)+3} \otimes e_{4 j}, \quad i=j \\
w_{4(j+m+s)+1 \rightarrow 4(j+m)+3} \otimes e_{4 j}, \quad i=j+s \\
0, \quad \text { otherwise. }
\end{array}\right.
$$

If $s \leqslant j<2 s$, then

$$
b_{i j}=\left\{\begin{array}{l}
w_{4(j+m)+1 \rightarrow 4(j+m+1)} \otimes e_{4 j}, \quad i=j ; \\
w_{4(j+m+s)+2 \rightarrow 4(j+m+1)} \otimes w_{4 j \rightarrow 4(j+s)+1}, \quad i=j+s \\
w_{4(j+m)+2 \rightarrow 4(j+m+1)} \otimes w_{4 j \rightarrow 4 j+1}, \quad i=j+2 s \\
0, \quad \text { otherwise. }
\end{array}\right.
$$

If $2 s \leqslant j<3 s$, then

$$
b_{i j}=\left\{\begin{array}{l}
w_{4(j+m)+2 \rightarrow 4(j+m+1)+1} \otimes e_{4 j+1}, \quad i=j \\
0, \quad \text { otherwise }
\end{array}\right.
$$

If $3 s \leqslant j<4 s$, then

$$
b_{i j}=\left\{\begin{array}{l}
w_{4(j+m)+2 \rightarrow 4(j+m+1)+1} \otimes e_{4 j+1}, \quad i=j \\
0, \quad \text { otherwise }
\end{array}\right.
$$

If $4 s \leqslant j<5 s$, then $b_{i j}=0$.

If $5 s \leqslant j<6 s$, then

$$
b_{i j}=\left\{\begin{array}{l}
w_{4(j+m)+3 \rightarrow 4(j+m+s+1)+2} \otimes e_{4(j+s)+2}, \quad i=j-s \\
w_{4(j+m+1) \rightarrow 4(j+m+s+1)+2} \otimes w_{4(j+s)+2 \rightarrow 4 j+3}, \quad i=j+s \\
0, \quad \text { otherwise. }
\end{array}\right.
$$

If $6 s \leqslant j<7 s$, then $b_{i j}=0$. 
If $7 s \leqslant j<8 s$, then

$$
b_{i j}=\left\{\begin{array}{l}
w_{4(j+m)+3 \rightarrow 4(j+m+1)+2} \otimes e_{4 j+2}, \quad i=j-2 s \\
w_{4(j+m+1) \rightarrow 4(j+m+1)+2} \otimes w_{4 j+2 \rightarrow 4 j+3}, \quad i=j-s \\
0, \quad \text { otherwise. }
\end{array}\right.
$$

If $8 s \leqslant j<9 s$, then

$$
b_{i j}=\left\{\begin{array}{l}
w_{4(j+m+1+f(j, 9 s-1) s)+1 \rightarrow 4(j+m+1)+3} \otimes w_{4 j+3 \rightarrow 4(j+1)}, \quad i=(j+1)_{s} ; \\
0, \quad \text { otherwise. }
\end{array}\right.
$$

(3) If $r_{0}=2$, then $\Omega^{2}\left(Y_{t}^{(7)}\right)$ is described with $(8 s \times 6 s)$-matrix with the following elements $b_{i j}$ : If $2 s \leqslant j<3 s$, then

$$
b_{i j}=\left\{\begin{array}{l}
w_{4(j+m)+2 \rightarrow 4(j+m+1)} \otimes e_{4 j+1}, \quad i=j-s \\
0, \quad \text { otherwise. }
\end{array}\right.
$$

If $3 s \leqslant j<4 s$, then

$$
b_{i j}=\left\{\begin{array}{l}
w_{4(j+m)+2 \rightarrow 4(j+m+1)} \otimes e_{4 j+1}, \quad i=j-s \\
0, \quad \text { otherwise. }
\end{array}\right.
$$

If $4 s \leqslant j<5 s$, then

$$
b_{i j}=\left\{\begin{array}{l}
w_{4(j+m+s)+1 \rightarrow 4(j+m)+3} \otimes e_{4 j+2}, \quad i=j-s \\
0, \quad \text { otherwise. }
\end{array}\right.
$$

If $5 s \leqslant j<6 s$, then

$$
b_{i j}=\left\{\begin{array}{l}
w_{4(j+m+s)+1 \rightarrow 4(j+m)+3} \otimes e_{4 j+2}, \quad i=j-s \\
0, \quad \text { otherwise. }
\end{array}\right.
$$

If $6 s \leqslant j<7 s-1$, then $b_{i j}=0$.

If $7 s-1 \leqslant j<7 s$, then

$$
b_{i j}=\left\{\begin{array}{l}
e_{4(j+m+s+1)+2} \otimes w_{4 j+3 \rightarrow 4(j+s+1)+1}, \quad i=s+(j+1)_{s} ; \\
0, \quad \text { otherwise. }
\end{array}\right.
$$

If $7 s \leqslant j<6 s$, then $b_{i j}=0$.

If $6 s \leqslant j<7 s-1$, then

$$
b_{i j}=\left\{\begin{array}{l}
e_{4(j+m+s+1)+2} \otimes w_{4 j+3 \rightarrow 4(j+s+1)+1}, \quad i=2 s+(j+1)_{s} \\
0, \quad \text { otherwise. }
\end{array}\right.
$$

If $7 s-1 \leqslant j<7 s$, then $b_{i j}=0$. 
HOCHSCHILD COHOMOLOGY RING FOR SELF-INJECTIVE ALGEBRAS OF TREE CLASS $E_{6}$. II. 67 If $7 s \leqslant j<8 s$, then

$$
b_{i j}=\left\{\begin{array}{l}
e_{4(j+m+s+1)+2} \otimes w_{4 j+3 \rightarrow 4(j+s+1)+1}, \quad i=s+f(j, 8 s-1) s+(j+1)_{s} \\
0, \quad \text { otherwise. }
\end{array}\right.
$$

(4) If $r_{0}=3$, then $\Omega^{3}\left(Y_{t}^{(7)}\right)$ is described with $(9 s \times 8 s)$-matrix with the following elements $b_{i j}$ : If $0 \leqslant j<s$, then

$$
b_{i j}=\left\{\begin{array}{l}
w_{4(j+m)+2 \rightarrow 4(j+m+1)} \otimes e_{4 j}, \quad i=j \\
w_{4(j+m+s)+2 \rightarrow 4(j+m+1)} \otimes e_{4 j}, \quad i=j+s \\
0, \quad \text { otherwise. }
\end{array}\right.
$$

If $s \leqslant j<2 s$, then $b_{i j}=0$.

If $2 s \leqslant j<3 s$, then

$$
b_{i j}=\left\{\begin{array}{l}
e_{4(j+m+1)+2} \otimes w_{4 j+1 \rightarrow 4(j+1)}, \quad i=f(j, 3 s-1) s+(j+1)_{s} \\
w_{4(j+m)+3 \rightarrow 4(j+m+1)+2} \otimes e_{4 j+1}, \quad i=j \\
0, \quad \text { otherwise. }
\end{array}\right.
$$

If $3 s \leqslant j<4 s$, then $b_{i j}=0$.

If $4 s \leqslant j<5 s$, then

$$
b_{i j}=\left\{\begin{array}{l}
e_{4(j+m+s+1)+2} \otimes w_{4(j+s)+1 \rightarrow 4(j+1)}, \quad i=s-f(j, 5 s-1) s+(j+1)_{s} \\
w_{4(j+m)+3 \rightarrow 4(j+m+s+1)+2} \otimes e_{4(j+s)+1}, \quad i=j-s \\
0, \quad \text { otherwise. }
\end{array}\right.
$$

If $5 s \leqslant j<7 s$, then $b_{i j}=0$.

If $7 s \leqslant j<8 s$, then

$$
b_{i j}=\left\{\begin{array}{l}
w_{4(j+m+s-f(j, 8 s-1) s+1)+2 \rightarrow 4(j+m+1)+3} \otimes w_{4 j+3 \rightarrow 4(j+1)}, \quad i=(j+1)_{s} \\
w_{4(j+m-f(j, 8 s-1) s+1)+2 \rightarrow 4(j+m+1)+3} \otimes w_{4 j+3 \rightarrow 4(j+1)}, \quad i=s+(j+1)_{s} \\
0, \quad \text { otherwise. }
\end{array}\right.
$$

If $8 s \leqslant j<9 s$, then $b_{i j}=0$.

(5) If $r_{0}=4$, then $\Omega^{4}\left(Y_{t}^{(7)}\right)$ is described with $(8 s \times 9 s)$-matrix with the following elements $b_{i j}$ : If $0 \leqslant j<s$, then

$$
b_{i j}=\left\{\begin{array}{l}
w_{4(j+m+s)-1 \rightarrow 4(j+m+s)+2} \otimes e_{4 j}, \quad i=j \\
w_{4(j+m+s)+1 \rightarrow 4(j+m+s)+2} \otimes w_{4 j \rightarrow 4 j+1}, \quad i=j+2 s \\
e_{4(j+m+s)+2} \otimes w_{4 j \rightarrow 4 j+2}, \quad i=j+5 s \\
0, \quad \text { otherwise. }
\end{array}\right.
$$


If $s \leqslant j<2 s$, then

$$
b_{i j}=\left\{\begin{array}{l}
w_{4(j+m+s)-1 \rightarrow 4(j+m+s)+2} \otimes e_{4 j}, \quad i=j-s \\
w_{4(j+m+s)+1 \rightarrow 4(j+m+s)+2} \otimes w_{4 j \rightarrow 4 j+1}, \quad i=j+2 s \\
e_{4(j+m+s)+2} \otimes w_{4 j \rightarrow 4 j+2}, \quad i=j+6 s \\
0, \quad \text { otherwise. }
\end{array}\right.
$$

If $2 s \leqslant j<3 s$, then

$$
b_{i j}=\left\{\begin{array}{l}
w_{4(j+m+s)+1 \rightarrow 4(j+m)+3} \otimes e_{4 j+1}, \quad i=j ; \\
w_{4(j+m+s)+2 \rightarrow 4(j+m)+3} \otimes w_{4 j+1 \rightarrow 4 j+2}, \quad i=j+3 s \\
0, \quad \text { otherwise. }
\end{array}\right.
$$

If $3 s \leqslant j<4 s$, then

$$
b_{i j}=\left\{\begin{array}{l}
w_{4(j+m+s)+1 \rightarrow 4(j+m)+3} \otimes e_{4 j+1}, \quad i=j ; \\
w_{4(j+m+s)+2 \rightarrow 4(j+m)+3} \otimes w_{4 j+1 \rightarrow 4 j+2}, \quad i=j+4 s \\
0, \quad \text { otherwise }
\end{array}\right.
$$

If $4 s \leqslant j<8 s$, then $b_{i j}=0$.

(6) If $r_{0}=5$, then $\Omega^{5}\left(Y_{t}^{(7)}\right)$ is described with $(6 s \times 8 s)$-matrix with the following elements $b_{i j}$ : If $s \leqslant j<2 s$, then

$$
b_{i j}=\left\{\begin{array}{l}
w_{4(j+m+1)+1 \rightarrow 4(j+m+1)+2} \otimes w_{4(j+s)+1 \rightarrow 4(j+1)}, \quad i=s-f(j, 2 s-1) s+(j+1)_{s} \\
w_{4(j+m+1) \rightarrow 4(j+m+1)+2} \otimes e_{4(j+s)+1}, \quad i=j+s \\
0, \quad \text { otherwise. }
\end{array}\right.
$$

If $2 s \leqslant j<3 s$, then

$$
b_{i j}=\left\{\begin{array}{l}
w_{4(j+m+1)+1 \rightarrow 4(j+m+1)+2} \otimes w_{4(j+s)+1 \rightarrow 4(j+1)}, \quad i=f(j, 3 s-1) s+(j+1)_{s} ; \\
w_{4(j+m+1) \rightarrow 4(j+m+1)+2} \otimes e_{4(j+s)+1}, \quad i=j+s ; \\
0, \quad \text { otherwise. }
\end{array}\right.
$$

If $3 s \leqslant j<5 s$, then $b_{i j}=0$.

If $5 s \leqslant j<6 s$, then

$$
b_{i j}=\left\{\begin{array}{l}
e_{4(j+m+2)} \otimes w_{4 j+3 \rightarrow 4(j+s-f(j, 6 s-1) s+1)+1}, \quad i=2 s+(j+1)_{s} ; \\
e_{4(j+m+2)} \otimes w_{4 j+3 \rightarrow 4(j+1+f(j, 6 s-1) s)+1}, \quad i=3 s+(j+1)_{s} ; \\
0, \quad \text { otherwise. }
\end{array}\right.
$$

(7) If $r_{0}=6$, then $\Omega^{6}\left(Y_{t}^{(7)}\right)$ is described with $(7 s \times 9 s)$-matrix with the following elements $b_{i j}$ : 
If $0 \leqslant j<s$, then

$$
b_{i j}=\left\{\begin{array}{l}
w_{4(j+m)+1 \rightarrow 4(j+m)+3} \otimes w_{4 j \rightarrow 4 j+1}, \quad i=j+s \\
w_{4(j+m+s)+1 \rightarrow 4(j+m)+3} \otimes w_{4 j \rightarrow 4(j+s)+1}, \quad i=j+3 s \\
0, \quad \text { otherwise. }
\end{array}\right.
$$

If $s \leqslant j<2 s$, then

$$
b_{i j}=\left\{\begin{array}{l}
e_{4(j+m+1)} \otimes w_{4(j+s)+1 \rightarrow 4(j+1)}, \quad i=(j+1)_{s} \\
w_{4(j+m+s)+1 \rightarrow 4(j+m+1)} \otimes e_{4(j+s)+1}, \quad i=j \\
0, \quad \text { otherwise. }
\end{array}\right.
$$

If $2 s \leqslant j<3 s$, then

$$
b_{i j}=\left\{\begin{array}{l}
e_{4(j+m+1)} \otimes w_{4(j+s)+1 \rightarrow 4(j+1)}, \quad i=(j+1)_{s} \\
w_{4(j+m+s)+1 \rightarrow 4(j+m+1)} \otimes e_{4(j+s)+1}, \quad i=j+s \\
0, \quad \text { otherwise. }
\end{array}\right.
$$

If $3 s \leqslant j<4 s$, then

$$
b_{i j}=\left\{\begin{array}{l}
e_{4(j+m+s+1)+1} \otimes w_{4(j+s)+2 \rightarrow 4(j+s+1)+1}, \quad i=s+f(j, 4 s-1) 2 s+(j+1)_{s} ; \\
0, \quad \text { otherwise. }
\end{array}\right.
$$

If $4 s \leqslant j<5 s$, then

$$
b_{i j}=\left\{\begin{array}{l}
e_{4(j+m+s+1)+1} \otimes w_{4(j+s)+2 \rightarrow 4(j+s+1)+1}, \quad i=3 s-f(j, 5 s-1) 2 s+(j+1)_{s} \\
0, \quad \text { otherwise. }
\end{array}\right.
$$

If $5 s \leqslant j<6 s$, then

$$
b_{i j}=\left\{\begin{array}{l}
w_{4(j+m+1)+1 \rightarrow 4(j+m+1)+2} \otimes w_{4 j+3 \rightarrow 4(j+1)+1}, \quad i=3 s-f(j, 6 s-1) 2 s+(j+1)_{s} \\
0, \quad \text { otherwise. }
\end{array}\right.
$$

If $6 s \leqslant j<7 s$, then

$$
b_{i j}=\left\{\begin{array}{l}
w_{4(j+m+1)+1 \rightarrow 4(j+m+1)+2} \otimes w_{4 j+3 \rightarrow 4(j+1)+1}, \quad i=s+f(j, 7 s-1) 2 s+(j+1)_{s} ; \\
0, \quad \text { otherwise. }
\end{array}\right.
$$

(8) If $r_{0}=7$, then $\Omega^{7}\left(Y_{t}^{(7)}\right)$ is described with $(6 s \times 8 s)$-matrix with the following elements $b_{i j}$ : If $s \leqslant j<2 s$, then

$$
b_{i j}=\left\{\begin{array}{l}
w_{4(j+m)+3 \rightarrow 4(j+m+s+1)+1} \otimes e_{4(j+s)+1}, \quad i=j+s \\
w_{4(j+m+1) \rightarrow 4(j+m+s+1)+1} \otimes w_{4(j+s)+1 \rightarrow 4(j+s)+2}, \quad i=j+3 s \\
e_{4(j+m+s+1)+1} \otimes w_{4(j+s)+1 \rightarrow 4 j+3}, \quad i=j+5 s \\
0, \quad \text { otherwise. }
\end{array}\right.
$$


If $2 s \leqslant j<3 s$, then

$$
b_{i j}=\left\{\begin{array}{l}
w_{4(j+m)+3 \rightarrow 4(j+m+s+1)+1} \otimes e_{4(j+s)+1}, \quad i=j+s \\
w_{4(j+m+1) \rightarrow 4(j+m+s+1)+1} \otimes w_{4(j+s)+1 \rightarrow 4(j+s)+2}, \quad i=j+3 s \\
e_{4(j+m+s+1)+1} \otimes w_{4(j+s)+1 \rightarrow 4 j+3}, \quad i=j+5 s \\
0, \quad \text { otherwise. }
\end{array}\right.
$$

If $3 s \leqslant j<6 s$, then $b_{i j}=0$.

(9) If $r_{0}=8$, then $\Omega^{8}\left(Y_{t}^{(7)}\right)$ is described with $(6 s \times 6 s)$-matrix with the following elements $b_{i j}$ : If $0 \leqslant j<s$, then

$$
b_{i j}=\left\{\begin{array}{l}
w_{4(j+m)+3 \rightarrow 4(j+m+1)} \otimes w_{4 j \rightarrow 4(j+1)}, \quad i=(j+1)_{s} \\
w_{4(j+m)+2 \rightarrow 4(j+m+1)} \otimes w_{4 j \rightarrow 4 j+1}, \quad i=j+s \\
w_{4(j+m+s)+2 \rightarrow 4(j+m+1)} \otimes w_{4 j \rightarrow 4(j+s)+1}, \quad i=j+2 s \\
0, \quad \text { otherwise. }
\end{array}\right.
$$

If $s \leqslant j<2 s$, then

$$
b_{i j}=\left\{\begin{array}{l}
w_{4(j+m)+3 \rightarrow 4(j+m+s+1)+1} \otimes w_{4(j+s)+1 \rightarrow 4(j+1)}, \quad i=(j+1)_{s} \\
w_{4(j+m+s)+2 \rightarrow 4(j+m+s+1)+1} \otimes e_{4(j+s)+1}, \quad i=j \\
0, \quad \text { otherwise. }
\end{array}\right.
$$

If $2 s \leqslant j<3 s$, then

$$
b_{i j}=\left\{\begin{array}{l}
w_{4(j+m)+3 \rightarrow 4(j+m+s+1)+1} \otimes w_{4(j+s)+1 \rightarrow 4(j+1)}, \quad i=(j+1)_{s} \\
w_{4(j+m+s)+2 \rightarrow 4(j+m+s+1)+1} \otimes e_{4(j+s)+1}, \quad i=j \\
0, \quad \text { otherwise. }
\end{array}\right.
$$

If $3 s \leqslant j<4 s$, then

$$
b_{i j}=\left\{\begin{array}{l}
e_{4(j+m+s+1)+2} \otimes w_{4(j+s)+2 \rightarrow 4(j+s+1)+1}, \quad i=s+f(j, 4 s-1) s+(j+1)_{s} \\
0, \quad \text { otherwise. }
\end{array}\right.
$$

If $4 s \leqslant j<5 s$, then

$$
b_{i j}=\left\{\begin{array}{l}
e_{4(j+m+s+1)+2} \otimes w_{4(j+s)+2 \rightarrow 4(j+s+1)+1}, \quad i=2 s-f(j, 5 s-1) s+(j+1)_{s} ; \\
0, \quad \text { otherwise. }
\end{array}\right.
$$

If $5 s \leqslant j<6 s$, then

$b_{i j}=\left\{\begin{array}{l}w_{4(j+m+s-f(j, 6 s-1) s+1)+2 \rightarrow 4(j+m+1)+3} \otimes w_{4 j+3 \rightarrow 4(j+s-f(j, 6 s-1) s+1)+1}, \quad i=s+(j+1)_{s} ; \\ w_{4(j+m+f(j, 6 s-1) s+1)+2 \rightarrow 4(j+m+1)+3} \otimes w_{4 j+3 \rightarrow 4(j+f(j, 6 s-1) s+1)+1}, \quad i=2 s+(j+1)_{s} ; \\ 0, \quad \text { otherwise. }\end{array}\right.$

(10) If $r_{0}=9$, then $\Omega^{9}\left(Y_{t}^{(7)}\right)$ is described with $(7 s \times 7 s)$-matrix with the following elements $b_{i j}$ : 
HOCHSCHILD COHOMOLOGY RING FOR SELF-INJECTIVE ALGEBRAS OF TREE CLASS $E_{6}$. II. 7 If $2 s \leqslant j<3 s$, then

$$
b_{i j}=\left\{\begin{array}{l}
w_{4(j+m+1) \rightarrow 4(j+m+1)+2} \otimes e_{4 j+1}, \quad i=j-s \\
e_{4(j+m+1)+2} \otimes w_{4 j+1 \rightarrow 4 j+3}, \quad i=j+3 s \\
0, \quad \text { otherwise. }
\end{array}\right.
$$

If $3 s \leqslant j<4 s$, then

$$
b_{i j}=\left\{\begin{array}{l}
w_{4(j+m+1) \rightarrow 4(j+m+1)+2} \otimes e_{4 j+1}, \quad i=j-s \\
e_{4(j+m+1)+2} \otimes w_{4 j+1 \rightarrow 4 j+3}, \quad i=j+3 s \\
0, \quad \text { otherwise. }
\end{array}\right.
$$

If $4 s \leqslant j<7 s$, then $b_{i j}=0$.

(11) If $r_{0}=10$, then $\Omega^{10}\left(Y_{t}^{(7)}\right)$ is described with $(6 s \times 6 s)$-matrix with the following elements $b_{i j}$ :

If $0 \leqslant j<s$, then

$$
b_{i j}= \begin{cases}w_{4(j+m+s)+1 \rightarrow 4(j+m)+3} \otimes w_{4 j \rightarrow 4 j+1}, & i=j+s \\ w_{4(j+m)+1 \rightarrow 4(j+m)+3} \otimes w_{4 j \rightarrow 4(j+s)+1}, & i=j+2 s \\ w_{4(j+m+s)+2 \rightarrow 4(j+m)+3} \otimes w_{4 j \rightarrow 4 j+2}, & i=j+3 s ; \\ w_{4(j+m)+2 \rightarrow 4(j+m)+3} \otimes w_{4 j \rightarrow 4(j+s)+2}, & i=j+4 s \\ 0, \quad \text { otherwise. } & \end{cases}
$$

If $s \leqslant j<2 s$, then

$$
b_{i j}=\left\{\begin{array}{l}
w_{4(j+m+1) \rightarrow 4(j+m+s+1)+2} \otimes w_{4(j+s)+1 \rightarrow 4(j+1)}, \quad i=(j+1)_{s} \\
w_{4(j+m)+3 \rightarrow 4(j+m+s+1)+2} \otimes w_{4(j+s)+1 \rightarrow 4 j+3}, \quad i=j+4 s \\
0, \quad \text { otherwise. }
\end{array}\right.
$$

If $2 s \leqslant j<3 s$, then

$$
b_{i j}=\left\{\begin{array}{l}
w_{4(j+m+1) \rightarrow 4(j+m+s+1)+2} \otimes w_{4(j+s)+1 \rightarrow 4(j+1)}, \quad i=(j+1)_{s} \\
w_{4(j+m)+3 \rightarrow 4(j+m+s+1)+2} \otimes w_{4(j+s)+1 \rightarrow 4 j+3}, \quad i=j+3 s \\
0, \quad \text { otherwise. }
\end{array}\right.
$$

If $3 s \leqslant j<5 s$, then $b_{i j}=0$.

If $5 s \leqslant j<6 s$, then

$$
b_{i j}= \begin{cases}w_{4(j+m+1) \rightarrow 4(j+m+2)} \otimes w_{4 j+3 \rightarrow 4(j+1)}, \quad i=(j+1)_{s} ; \\ w_{4(j+m+s-f(j, 6 s-1) s+1)+1 \rightarrow 4(j+m+2)} \otimes w_{4 j+3 \rightarrow 4(j+f(j, 6 s-1) s+1)+1}, \quad i=2 s+(j+1)_{s} ; \\ w_{4(j+m+s-f(j, 6 s-1) s+1)+2 \rightarrow 4(j+m+2)} \otimes w_{4 j+3 \rightarrow 4(j+f(j, 6 s-1) s+1)+2,} i=4 s+(j+1)_{s} ; \\ 0, \quad \text { otherwise. }\end{cases}
$$

(II) Represent an arbitrary $t_{0} \in \mathbb{N}$ in the form $t_{0}=11 \ell_{0}+r_{0}$, where $0 \leqslant r_{0} \leqslant 10$. Then $\Omega^{t_{0}}\left(Y_{t}^{(7)}\right)$ is a $\Omega^{r_{0}}\left(Y_{t}^{(7)}\right)$, whose left components twisted by $\sigma^{\ell_{0}}$. 
Proposition 24 (Translates for the case 8). (I) Let $r_{0} \in \mathbb{N}, r_{0}<11 . r_{0}$-translates of the elements $Y_{t}^{(8)}$ are described by the following way.

(1) If $r_{0}=0$, then $\Omega^{0}\left(Y_{t}^{(8)}\right)$ is described with $(9 s \times 6 s)$-matrix with one nonzero element that is of the following form:

$$
b_{0,0}=w_{4(j+m) \rightarrow 4(j+m)+3} \otimes e_{4 j} .
$$

(2) If $r_{0}=1$, then $\Omega^{1}\left(Y_{t}^{(8)}\right)$ is described with $(8 s \times 7 s)$-matrix with the following two nonzero elements:

$$
\begin{gathered}
b_{s+(j+1)_{s}, 4 s-1}=w_{4(j+m+s+1)+1 \rightarrow 4(j+m+2)} \otimes w_{4 j+1 \rightarrow 4(j+1)} ; \\
b_{s+(j+1)_{s}, 6 s-1}=w_{4(j+m+s+1)+1 \rightarrow 4(j+m+1)+3} \otimes w_{4 j+2 \rightarrow 4(j+1)} .
\end{gathered}
$$

(3) If $r_{0}=2$, then $\Omega^{2}\left(Y_{t}^{(8)}\right)$ is described with $(9 s \times 6 s)$-matrix with one nonzero element that is of the following form:

$$
b_{(j+1)_{s}, 4 s-1}=w_{4(j+m)+3 \rightarrow 4(j+m+1+s)+1} \otimes w_{4 j+1 \rightarrow 4(j+1)} .
$$

(4) If $r_{0}=3$, then $\Omega^{3}\left(Y_{t}^{(8)}\right)$ is described with $(8 s \times 8 s)$-matrix with the following two nonzero elements:

$$
\begin{gathered}
b_{s+(j+1)_{s}, 2 s-1}=e_{4(j+m+s+1)+2} \otimes w_{4 j \rightarrow 4(j+1)} ; \\
b_{s+(j+1)_{s}, 4 s-1}=w_{4(j+m+s+1)+2 \rightarrow 4(j+m+1)+3} \otimes w_{4 j+1 \rightarrow 4(j+1)} .
\end{gathered}
$$

(5) If $r_{0}=4$, then $\Omega^{4}\left(Y_{t}^{(8)}\right)$ is described with $(6 s \times 9 s)$-matrix with the following two nonzero elements:

$$
\begin{gathered}
b_{(j+1)_{s}, 3 s-1}=w_{4(j+m)+3 \rightarrow 4(j+m+1)+2} \otimes w_{4(j+s)+1 \rightarrow 4(j+1)} ; \\
b_{s+(j+1)_{s}, 3 s-1}=w_{4(j+m+1) \rightarrow 4(j+m+1)+2} \otimes w_{4(j+s)+1 \rightarrow 4(j+1)} .
\end{gathered}
$$

(6) If $r_{0}=5$, then $\Omega^{5}\left(Y_{t}^{(8)}\right)$ is described with $(7 s \times 8 s)$-matrix with the following two nonzero elements:

$$
\begin{gathered}
b_{(j+1)_{s}, s-1}=w_{4(j+m+1+s f(j, s-1))+1 \rightarrow 4(j+m+1)+3} \otimes w_{4 j \rightarrow 4(j+1)} ; \\
b_{(j+1)_{s}, 3 s-1}=w_{4(j+m+s+1)+1 \rightarrow 4(j+m+2)} \otimes w_{4(j+s)+1 \rightarrow 4(j+1)} .
\end{gathered}
$$

(7) If $r_{0}=6$, then $\Omega^{6}\left(Y_{t}^{(8)}\right)$ is described with $(6 s \times 9 s)$-matrix with the following two nonzero elements:

$$
\begin{gathered}
b_{(j+1)_{s}, 3 s-1}=w_{4(j+m+1) \rightarrow 4(j+m+s+1)+1} \otimes w_{4(j+s)+1 \rightarrow 4(j+1)} \\
b_{s+(j+1)_{s}, 3 s-1}=e_{4(j+m+s+1)+1} \otimes w_{4(j+s)+1 \rightarrow 4(j+s+1)+1} .
\end{gathered}
$$

(8) If $r_{0}=7$, then $\Omega^{7}\left(Y_{t}^{(8)}\right)$ is described with $(6 s \times 8 s)$-matrix with the following two nonzero elements:

$$
\begin{gathered}
b_{(j+1)_{s}, s-1}=w_{4(j+m+1+s f(j, s-1))+2 \rightarrow 4(j+m+2)} \otimes w_{4 j \rightarrow 4(j+1)} ; \\
b_{(j+1)_{s}, 3 s-1}=w_{4(j+m+s+1)+2 \rightarrow 4(j+m+s+2)+1} \otimes w_{4(j+s)+1 \rightarrow 4(j+1)} .
\end{gathered}
$$

(9) If $r_{0}=8$, then $\Omega^{8}\left(Y_{t}^{(8)}\right)$ is described with $(7 s \times 6 s)$-matrix with the following two nonzero elements:

$$
\begin{gathered}
b_{(j+1)_{s}, 4 s-1}=w_{4(j+m)+3 \rightarrow 4(j+m+1)+2} \otimes w_{4 j+1 \rightarrow 4(j+1)} \\
b_{s+(j+1)_{s}, 4 s-1}=e_{4(j+m+1)+2} \otimes w_{4 j+1 \rightarrow 4(j+1)+1} .
\end{gathered}
$$

(10) If $r_{0}=9$, then $\Omega^{9}\left(Y_{t}^{(8)}\right)$ is described with $(6 s \times 7 s)$-matrix with the following nonzero elements:

$$
\begin{gathered}
b_{j+s, s-1}=w_{4(j+m+1) \rightarrow 4(j+m+1)+3} \otimes w_{4 j \rightarrow 4 j+1} ; \\
b_{j+5 s, s-1}=w_{4(j+m+1)+2 \rightarrow 4(j+m)+3} \otimes w_{4 j \rightarrow 4 j+3}, \quad s=1
\end{gathered}
$$




$$
\begin{gathered}
b_{j+2 s, s-1}=w_{4(j+m+1) \rightarrow 4(j+m+1)+3} \otimes w_{4 j \rightarrow 4(j+s)+1}, \quad s>1 ; \\
b_{(j+1)_{s}, 3 s-1}=w_{4(j+m+1)+3 \rightarrow 4(j+m+s+2)+2} \otimes w_{4(j+s)+1 \rightarrow 4(j+1)} .
\end{gathered}
$$

(11) If $r_{0}=10$ and $s=1$, then $\Omega^{10}\left(Y_{t}^{(8)}\right)$ is described with $(8 s \times 6 s)$-matrix with the following nonzero elements:

$$
\begin{gathered}
b_{j, 0}=w_{4(j+m) \rightarrow 4(j+m+1)+2} \otimes e_{4 j} ; \\
b_{j+5 s, 0}=w_{4(j+m)+3 \rightarrow 4(j+m+1)+2} \otimes w_{4 j \rightarrow 4 j+3} ; \\
b_{j-s, s}=w_{4(j+m) \rightarrow 4(j+m+1)+2} \otimes e_{4 j} ; \\
b_{j-3 s, 3 s}=w_{4(j+m) \rightarrow 4(j+m)+3} \otimes w_{4 j+1 \rightarrow 4 j} ; \\
b_{j-5 s, 5 s}=e_{4(j+m)} \otimes w_{4 j+2 \rightarrow 4 j} ; \\
b_{j-2 s, 7 s}=w_{4(j+m)+3 \rightarrow 4(j+m)+1} \otimes e_{4 j+3} .
\end{gathered}
$$

(12) If $r_{0}=10$ and $s>1$, then $\Omega^{10}\left(Y_{t}^{(8)}\right)$ is described with $(8 s \times 6 s)$-matrix with the following nonzero elements:

$$
\begin{gathered}
b_{j+2 s, 2 s-1}=w_{4(j+m)+2 \rightarrow 4(j+m+1)+2} \otimes w_{4 j \rightarrow 4(j+s)+2} ; \\
b_{j+4 s, 2 s-1}=w_{4(j+m)+3 \rightarrow 4(j+m+1)+2} \otimes w_{4 j \rightarrow 4 j+3} ; \\
b_{s+(j+1)_{s}, 3 s-2}=w_{4(j+m+s+1)+1 \rightarrow 4(j+m+1)+3} \otimes w_{4 j+1 \rightarrow 4(j+1)+1} ; \\
b_{(j+1)_{s}, 4 s-2}=w_{4(j+m+1) \rightarrow 4(j+m+1)+3} \otimes w_{4 j+1 \rightarrow 4(j+1)} ; \\
b_{j+2 s, 4 s-2}=w_{4(j+m)+3 \rightarrow 4(j+m+1)+3} \otimes w_{4 j+1 \rightarrow 4 j+3} ; \\
b_{j+2 s, 4 s-1}=w_{4(j+m)+3 \rightarrow 4(j+m+1)+3} \otimes w_{4 j+1 \rightarrow 4 j+3} ; \\
b_{s+(j+1)_{s}, 5 s-2}=w_{4(j+m+s+1)+1 \rightarrow 4(j+m+2)} \otimes w_{4 j+2 \rightarrow 4(j+1)+1} ; \\
b_{3 s+(j+1)_{s}, 5 s-2}=w_{4(j+m+s+1)+2 \rightarrow 4(j+m+2)} \otimes w_{4 j+2 \rightarrow 4(j+1)+2} .
\end{gathered}
$$

(II) Represent an arbitrary $t_{0} \in \mathbb{N}$ in the form $t_{0}=11 \ell_{0}+r_{0}$, where $0 \leqslant r_{0} \leqslant 10$. Then $\Omega^{t_{0}}\left(Y_{t}^{(8)}\right)$ is a $\Omega^{r_{0}}\left(Y_{t}^{(8)}\right)$, whose left components twisted by $\sigma^{\ell_{0}}$.

Proposition 25 (Translates for the case 9). (I) Let $r_{0} \in \mathbb{N}, r_{0}<11$. $r_{0}$-translates of the elements $Y_{t}^{(9)}$ are described by the following way.

(1) If $r_{0}=0$, then $\Omega^{0}\left(Y_{t}^{(9)}\right)$ is described with $(9 s \times 6 s)$-matrix with the following elements $b_{i j}$ : If $s \leqslant j<2 s$, then

$$
b_{i j}=\left\{\begin{array}{l}
\kappa^{\ell}\left(\alpha_{3(j+m+2)}\right) e_{4 j} \otimes e_{4 j}, \quad i=j-s \\
0, \quad \text { otherwise }
\end{array}\right.
$$

If $2 s \leqslant j<4 s$, then

$$
b_{i j}=\left\{\begin{array}{l}
e_{4 j+1} \otimes e_{4 j+1}, \quad i=j-s \\
0, \quad \text { otherwise. }
\end{array}\right.
$$

If $4 s \leqslant j<5 s$, then $b_{i j}=0$.

If $5 s \leqslant j<6 s$, then

$$
b_{i j}=\left\{\begin{array}{l}
e_{4(j+s)+2} \otimes e_{4(j+s)+2}, \quad i=j-2 s ; \\
0, \quad \text { otherwise. }
\end{array}\right.
$$

If $6 s \leqslant j<7 s$, then $b_{i j}=0$. 
If $7 s \leqslant j<8 s$, then

$$
b_{i j}=\left\{\begin{array}{l}
e_{4 j+2} \otimes e_{4 j+2}, \quad i=j-3 s \\
0, \quad \text { otherwise. }
\end{array}\right.
$$

If $8 s \leqslant j<9 s$, then

$$
b_{i j}=\left\{\begin{array}{l}
-\kappa^{\ell}\left(\alpha_{3(j+m+2)}\right) e_{4 j+3} \otimes e_{4 j+3}, \quad i=j-3 s \\
0, \quad \text { otherwise. }
\end{array}\right.
$$

(2) If $r_{0}=1$, then $\Omega^{1}\left(Y_{t}^{(9)}\right)$ is described with $(8 s \times 7 s)$-matrix with the following elements $b_{i j}$ : If $0 \leqslant j<s$, then

$$
b_{i j}=\left\{\begin{array}{l}
e_{4(j+m+s)+1} \otimes e_{4 j}, \quad i=j+s \\
0, \quad \text { otherwise. }
\end{array}\right.
$$

If $s \leqslant j<2 s$, then

$$
b_{i j}=\left\{\begin{array}{l}
e_{4(j+m+s)+1} \otimes e_{4 j}, \quad i=j-s \\
0, \quad \text { otherwise }
\end{array}\right.
$$

If $2 s \leqslant j<3 s$, then

$$
b_{i j}=\left\{\begin{array}{l}
\kappa^{\ell}\left(\alpha_{3(j+m+3)}\right) w_{4(j+m)+2 \rightarrow 4(j+m+1)} \otimes e_{4 j+1}, \quad i=j ; \\
-\kappa^{\ell}\left(\alpha_{3(j+m+3)}\right) w_{4(j+m)+3 \rightarrow 4(j+m+1)} \otimes w_{4 j+1 \rightarrow 4 j+2}, \quad i=j+2 s ; \\
-\kappa^{\ell}\left(\alpha_{3(j+m+3)}\right) e_{4(j+m+1)} \otimes w_{4 j+1 \rightarrow 4 j+3}, \quad i=j+4 s \\
0, \quad \text { otherwise. }
\end{array}\right.
$$

If $3 s \leqslant j<4 s$, then

$$
b_{i j}=\left\{\begin{array}{l}
\kappa^{\ell}\left(\alpha_{3(j+m+3)}\right) w_{4(j+m)+2 \rightarrow 4(j+m+1)} \otimes e_{4 j+1}, \quad i=j ; \\
-\kappa^{\ell}\left(\alpha_{3(j+m+3)}\right) w_{4(j+m)+3 \rightarrow 4(j+m+1)} \otimes w_{4 j+1 \rightarrow 4 j+2}, \quad i=j+2 s ; \\
-\kappa^{\ell}\left(\alpha_{3(j+m+3)}\right) e_{4(j+m+1)} \otimes w_{4 j+1 \rightarrow 4 j+3}, \quad i=j+3 s ; \\
0, \quad \text { otherwise. }
\end{array}\right.
$$

If $4 s \leqslant j<5 s$, then

$$
b_{i j}=\left\{\begin{array}{l}
-\kappa^{\ell}\left(\alpha_{3(j+m+2)}\right) e_{4(j+m)+3} \otimes e_{4 j+2}, \quad i=j ; \\
0, \quad \text { otherwise. }
\end{array}\right.
$$

If $5 s \leqslant j<6 s$, then

$$
b_{i j}=\left\{\begin{array}{l}
-\kappa^{\ell}\left(\alpha_{3(j+m+2)}\right) e_{4(j+m)+3} \otimes e_{4 j+2}, \quad i=j ; \\
0, \quad \text { otherwise. }
\end{array}\right.
$$


If $6 s \leqslant j<7 s$, then

$$
b_{i j}=\left\{\begin{array}{l}
-w_{4(j+m+s+1)+1 \rightarrow 4(j+m+s+1)+2} \otimes w_{4 j+3 \rightarrow 4(j+1)}, \quad i=(j+s+1)_{2 s} \\
e_{4(j+m+s+1)+2} \otimes w_{4 j+3 \rightarrow 4(j+s+1)+1}, \quad i=2 s+(j+s+1)_{2 s} \\
-w_{4(j+m+1) \rightarrow 4(j+m+s+1)+2} \otimes e_{4 j+3}, \quad i=j \\
0, \quad \text { otherwise. }
\end{array}\right.
$$

If $7 s \leqslant j<8 s$, then

$$
b_{i j}=\left\{\begin{array}{l}
w_{4(j+m+s+1)+1 \rightarrow 4(j+m+s+1)+2} \otimes w_{4 j+3 \rightarrow 4(j+1)}, \quad i=(j+s+1)_{2 s} ; \\
-e_{4(j+m+s+1)+2} \otimes w_{4 j+3 \rightarrow 4(j+s+1)+1}, \quad i=2 s+(j+s+1)_{2 s} ; \\
w_{4(j+m+1) \rightarrow 4(j+m+s+1)+2} \otimes e_{4 j+3}, \quad i=j-s ; \\
0, \quad \text { otherwise. }
\end{array}\right.
$$

(3) If $r_{0}=2$, then $\Omega^{2}\left(Y_{t}^{(9)}\right)$ is described with $(9 s \times 6 s)$-matrix with the following elements $b_{i j}$ : If $0 \leqslant j<s$, then

$$
b_{i j}=\left\{\begin{array}{l}
\kappa^{\ell}\left(\alpha_{3(j+m+2)}\right) w_{4(j+m)-1 \rightarrow 4(j+m)} \otimes e_{4 j}, \quad i=j ; \\
0, \quad \text { otherwise. }
\end{array}\right.
$$

If $s \leqslant j<2 s$, then

$$
b_{i j}=\left\{\begin{array}{l}
-e_{4(j+m)+1} \otimes w_{4(j+s)+1 \rightarrow 4(j+s)+2}, \quad i=j+2 s \\
0, \quad \text { otherwise. }
\end{array}\right.
$$

If $2 s \leqslant j<3 s$, then

$$
b_{i j}=\left\{\begin{array}{l}
e_{4(j+m)+2} \otimes e_{4 j+1}, \quad i=j-s \\
0, \quad \text { otherwise. }
\end{array}\right.
$$

If $3 s \leqslant j<4 s$, then

$$
b_{i j}=\left\{\begin{array}{l}
-e_{4(j+m+s)+1} \otimes w_{4 j+1 \rightarrow 4 j+2}, \quad i=j+s \\
0, \quad \text { otherwise. }
\end{array}\right.
$$

If $4 s \leqslant j<5 s$, then

$$
b_{i j}=\left\{\begin{array}{l}
e_{4(j+m+s)+2} \otimes e_{4(j+s)+1}, \quad i=j-2 s \\
0, \quad \text { otherwise }
\end{array}\right.
$$

If $5 s \leqslant j<6 s$, then

$$
b_{i j}=\left\{\begin{array}{l}
-w_{4(j+m)+1 \rightarrow 4(j+m)+2} \otimes e_{4(j+s)+2}, \quad i=j-2 s \\
0, \quad \text { otherwise }
\end{array}\right.
$$


If $6 s \leqslant j<7 s$, then

$$
b_{i j}=\left\{\begin{array}{l}
-w_{4(j+m)+1 \rightarrow 4(j+m)+2} \otimes e_{4(j+s)+2}, \quad i=j-2 s \\
0, \quad \text { otherwise. }
\end{array}\right.
$$

If $7 s \leqslant j<8 s$, then

$$
b_{i j}=\left\{\begin{array}{l}
\kappa^{\ell}\left(\alpha_{3(j+m+2)}\right) f_{2}(j, 8 s-1) e_{4(j+m)+3} \otimes w_{4 j+3 \rightarrow 4(j+1)}, \quad i=(j+1)_{s} ; \\
0, \quad \text { otherwise. }
\end{array}\right.
$$

If $8 s \leqslant j<9 s-1$, then

$$
b_{i j}=\left\{\begin{array}{l}
\kappa^{\ell}\left(\alpha_{3(j+m+3)}\right) w_{4(j+m)+3 \rightarrow 4(j+m+1)} \otimes w_{4 j+3 \rightarrow 4(j+1)}, \quad i=(j+1)_{s} ; \\
0, \quad \text { otherwise. }
\end{array}\right.
$$

If $9 s-1 \leqslant j<8 s$, then $b_{i j}=0$.

If $8 s \leqslant j<9 s$, then

$$
b_{i j}=\left\{\begin{array}{l}
-\kappa^{\ell}\left(\alpha_{3(j+m+3)}\right) e_{4(j+m+1)} \otimes e_{4 j+3}, \quad i=j-3 s \\
0, \quad \text { otherwise. }
\end{array}\right.
$$

(4) If $r_{0}=3$, then $\Omega^{3}\left(Y_{t}^{(9)}\right)$ is described with $(8 s \times 8 s)$-matrix with the following elements $b_{i j}$ : If $0 \leqslant j<s$, then

$$
b_{i j}=\left\{\begin{array}{l}
-e_{4(j+m+s)+2} \otimes e_{4 j}, \quad i=j+s \\
0, \quad \text { otherwise }
\end{array}\right.
$$

If $s \leqslant j<2 s$, then

$$
b_{i j}=\left\{\begin{array}{l}
e_{4(j+m+s)+2} \otimes e_{4 j}, \quad i=j-s \\
0, \quad \text { otherwise }
\end{array}\right.
$$

If $2 s \leqslant j<3 s$, then

$$
b_{i j}=\left\{\begin{array}{l}
-\kappa^{\ell}\left(\alpha_{3(j+m+2)}\right) e_{4(j+m)+3} \otimes e_{4 j+1}, \quad i=j ; \\
0, \quad \text { otherwise. }
\end{array}\right.
$$

If $3 s \leqslant j<4 s$, then

$$
b_{i j}=\left\{\begin{array}{l}
-\kappa^{\ell}\left(\alpha_{3(j+m+2)}\right) e_{4(j+m)+3} \otimes e_{4 j+1}, \quad i=j ; \\
0, \quad \text { otherwise. }
\end{array}\right.
$$

If $4 s \leqslant j<5 s$, then

$$
b_{i j}=\left\{\begin{array}{l}
-\kappa^{\ell}\left(\alpha_{3(j+m+3)}\right) e_{4(j+m+1)} \otimes e_{4 j+2}, \quad i=j ; \\
0, \quad \text { otherwise. }
\end{array}\right.
$$


HOCHSCHILD COHOMOLOGY RING FOR SELF-INJECTIVE ALGEBRAS OF TREE CLASS $E_{6}$. II. 77 If $5 s \leqslant j<6 s$, then

$$
b_{i j}=\left\{\begin{array}{l}
-\kappa^{\ell}\left(\alpha_{3(j+m+3)}\right) e_{4(j+m+1)} \otimes e_{4 j+2}, \quad i=j \\
0, \quad \text { otherwise. }
\end{array}\right.
$$

If $6 s \leqslant j<7 s$, then

$$
b_{i j}=\left\{\begin{array}{l}
e_{4(j+m+s+1)+1} \otimes e_{4 j+3}, \quad i=j+s \\
0, \quad \text { otherwise }
\end{array}\right.
$$

If $7 s \leqslant j<8 s$, then

$$
b_{i j}=\left\{\begin{array}{l}
e_{4(j+m+s+1)+1} \otimes e_{4 j+3}, \quad i=j-s \\
0, \quad \text { otherwise }
\end{array}\right.
$$

(5) If $r_{0}=4$, then $\Omega^{4}\left(Y_{t}^{(9)}\right)$ is described with $(6 s \times 9 s)$-matrix with the following elements $b_{i j}$ : If $0 \leqslant j<s$, then

$$
b_{i j}=\left\{\begin{array}{l}
-\kappa^{\ell}\left(\alpha_{3(j+m+1)}\right) e_{4(j+2)-1} \otimes e_{4 j}, \quad i=j \\
0, \quad \text { otherwise. }
\end{array}\right.
$$

If $s \leqslant j<2 s$, then

$$
b_{i j}= \begin{cases}-w_{4(j+m)+1 \rightarrow 4(j+m)+2} \otimes e_{4(j+s)+1}, & i=j+s \\ -e_{4(j+m)+2} \otimes w_{4(j+s)+1 \rightarrow 4(j+s)+2}, & i=j+4 s \\ 0, \quad \text { otherwise. } & \end{cases}
$$

If $2 s \leqslant j<3 s$, then

$$
b_{i j}= \begin{cases}-w_{4(j+m)+1 \rightarrow 4(j+m)+2} \otimes e_{4(j+s)+1}, & i=j+s \\ -e_{4(j+m)+2} \otimes w_{4(j+s)+1 \rightarrow 4(j+s)+2}, & i=j+5 s \\ 0, \quad \text { otherwise. } & \end{cases}
$$

If $3 s \leqslant j<4 s$, then

$$
b_{i j}=\left\{\begin{array}{l}
e_{4(j+m+s)+1} \otimes e_{4(j+s)+2}, \quad i=j+s \\
0, \quad \text { otherwise }
\end{array}\right.
$$

If $4 s \leqslant j<5 s$, then

$$
b_{i j}=\left\{\begin{array}{l}
e_{4(j+m+s)+1} \otimes e_{4(j+s)+2}, \quad i=j+2 s \\
0, \quad \text { otherwise }
\end{array}\right.
$$


If $5 s \leqslant j<6 s$, then

$$
b_{i j}=\left\{\begin{array}{l}
-\kappa^{\ell}\left(\alpha_{3(j+m+3)}\right) f_{1}(j, 6 s-1) e_{4(j+m+1)} \otimes w_{4 j+3 \rightarrow 4(j+1)}, \quad i=s+(j+1)_{s} \\
-\kappa^{\ell}\left(\alpha_{3(j+m+3)}\right) w_{4(j+m)+3 \rightarrow 4(j+m+1)} \otimes e_{4 j+3}, \quad i=j+3 s \\
0, \quad \text { otherwise. }
\end{array}\right.
$$

(6) If $r_{0}=5$, then $\Omega^{5}\left(Y_{t}^{(9)}\right)$ is described with $(7 s \times 8 s)$-matrix with the following elements $b_{i j}$ : If $0 \leqslant j<s$, then

$$
b_{i j}=\left\{\begin{array}{l}
\kappa^{\ell}\left(\alpha_{3(j+m+2)}\right) w_{4(j+m)+1 \rightarrow 4(j+m)+3} \otimes e_{4 j}, \quad i=j \\
\kappa^{\ell}\left(\alpha_{3(j+m+2)}\right) w_{4(j+m+s)+1 \rightarrow 4(j+m)+3} \otimes e_{4 j}, \quad i=j+s \\
\kappa^{\ell}\left(\alpha_{3(j+m+2)}\right) e_{4(j+m)+3} \otimes w_{4 j \rightarrow 4 j+2}, \quad i=j+4 s \\
\kappa^{\ell}\left(\alpha_{3(j+m+2)}\right) e_{4(j+m)+3} \otimes w_{4 j \rightarrow 4(j+s)+2}, \quad i=j+5 s \\
0, \quad \text { otherwise. }
\end{array}\right.
$$

If $s \leqslant j<2 s$, then

$$
b_{i j}=\left\{\begin{array}{l}
\kappa^{\ell}\left(\alpha_{3(j+m+3)}\right) e_{4(j+m+1)} \otimes e_{4(j+s)+1}, \quad i=j+s ; \\
\kappa^{\ell}\left(\alpha_{3(j+m+3)}\right) w_{4(j+m)+3 \rightarrow 4(j+m+1)} \otimes w_{4(j+s)+1 \rightarrow 4(j+s)+2}, \quad i=j+3 s \\
0, \quad \text { otherwise. }
\end{array}\right.
$$

If $2 s \leqslant j<3 s$, then

$$
b_{i j}=\left\{\begin{array}{l}
\kappa^{\ell}\left(\alpha_{3(j+m+3)}\right) e_{4(j+m+1)} \otimes e_{4(j+s)+1}, \quad i=j+s \\
\kappa^{\ell}\left(\alpha_{3(j+m+3)}\right) w_{4(j+m)+3 \rightarrow 4(j+m+1)} \otimes w_{4(j+s)+1 \rightarrow 4(j+s)+2}, \quad i=j+3 s \\
0, \quad \text { otherwise. }
\end{array}\right.
$$

If $3 s \leqslant j<4 s$, then

$$
b_{i j}=\left\{\begin{array}{l}
-e_{4(j+m+s+1)+1} \otimes w_{4(j+s)+2 \rightarrow 4(j+1)}, \quad i=(j+s+1)_{2 s} \\
-w_{4(j+m)+3 \rightarrow 4(j+m+s+1)+1} \otimes e_{4(j+s)+2}, \quad i=j+s \\
0, \quad \text { otherwise. }
\end{array}\right.
$$

If $4 s \leqslant j<5 s$, then

$$
b_{i j}=\left\{\begin{array}{l}
-e_{4(j+m+s+1)+1} \otimes w_{4(j+s)+2 \rightarrow 4(j+1),} \quad i=(j+s+1)_{2 s} \\
-w_{4(j+m)+3 \rightarrow 4(j+m+s+1)+1} \otimes e_{4(j+s)+2}, \quad i=j+s \\
0, \quad \text { otherwise. }
\end{array}\right.
$$

If $5 s \leqslant j<6 s$, then

$$
b_{i j}=\left\{\begin{array}{l}
-w_{4(j+m+1)+1 \rightarrow 4(j+m+1)+2} \otimes w_{4 j+3 \rightarrow 4(j+1)}, \quad i=(j+1)_{2 s} \\
-e_{4(j+m+1)+2} \otimes e_{4 j+3}, \quad i=j+2 s \\
0, \quad \text { otherwise. }
\end{array}\right.
$$


If $6 s \leqslant j<7 s$, then

$$
b_{i j}=\left\{\begin{array}{l}
-w_{4(j+m+1)+1 \rightarrow 4(j+m+1)+2} \otimes w_{4 j+3 \rightarrow 4(j+1)}, \quad i=(j+1)_{2 s} ; \\
e_{4(j+m+1)+2} \otimes e_{4 j+3}, \quad i=j \\
0, \quad \text { otherwise. }
\end{array}\right.
$$

(7) If $r_{0}=6$, then $\Omega^{6}\left(Y_{t}^{(9)}\right)$ is described with $(6 s \times 9 s)$-matrix with the following elements $b_{i j}$ : If $0 \leqslant j<s$, then

$$
b_{i j}=\left\{\begin{array}{l}
-\kappa^{\ell}\left(\alpha_{3(j+m+2)}\right) e_{4(j+m)} \otimes e_{4 j}, \quad i=j \\
0, \quad \text { otherwise. }
\end{array}\right.
$$

If $s \leqslant j<2 s$, then

$$
b_{i j}=\left\{\begin{array}{l}
e_{4(j+m+s)+1} \otimes e_{4(j+s)+1}, \quad i=j \\
0, \quad \text { otherwise }
\end{array}\right.
$$

If $2 s \leqslant j<3 s$, then

$$
b_{i j}=\left\{\begin{array}{l}
e_{4(j+m+s)+1} \otimes e_{4(j+s)+1}, \quad i=j+s \\
0, \quad \text { otherwise }
\end{array}\right.
$$

If $3 s \leqslant j<4 s$, then

$$
b_{i j}=\left\{\begin{array}{l}
e_{4(j+m+s)+2} \otimes e_{4(j+s)+2}, \quad i=j+2 s \\
0, \quad \text { otherwise }
\end{array}\right.
$$

If $4 s \leqslant j<5 s$, then

$$
b_{i j}=\left\{\begin{array}{l}
e_{4(j+m+s)+2} \otimes e_{4(j+s)+2}, \quad i=j+2 s \\
0, \quad \text { otherwise }
\end{array}\right.
$$

If $5 s \leqslant j<6 s$, then

$$
b_{i j}=\left\{\begin{array}{l}
\kappa^{\ell}\left(\alpha_{3(j+m+2)}\right) e_{4(j+m)+3} \otimes e_{4 j+3}, \quad i=j+2 s \\
0, \quad \text { otherwise. }
\end{array}\right.
$$

(8) If $r_{0}=7$, then $\Omega^{7}\left(Y_{t}^{(9)}\right)$ is described with $(6 s \times 8 s)$-matrix with the following elements $b_{i j}$ : If $0 \leqslant j<s$, then

$$
b_{i j}=\left\{\begin{array}{l}
\kappa^{\ell}\left(\alpha_{3(j+m+3)}\right) w_{4(j+m+s)+2 \rightarrow 4(j+m+1)} \otimes e_{4 j}, \quad i=j+s \\
-\kappa^{\ell}\left(\alpha_{3(j+m+3)}\right) w_{4(j+m)+3 \rightarrow 4(j+m+1)} \otimes w_{4 j \rightarrow 4 j+1}, \quad i=j+2 s \\
\kappa^{\ell}\left(\alpha_{3(j+m+3)}\right) e_{4(j+m+1)} \otimes w_{4 j \rightarrow 4 j+2}, \quad i=j+4 s \\
\kappa^{\ell}\left(\alpha_{3(j+m+3)}\right) e_{4(j+m+1)} \otimes w_{4 j \rightarrow 4(j+s)+2}, \quad i=j+5 s \\
0, \quad \text { otherwise. }
\end{array}\right.
$$


If $s \leqslant j<2 s$, then

$$
b_{i j}=\left\{\begin{array}{l}
-w_{4(j+m)+3 \rightarrow 4(j+m+s+1)+1} \otimes e_{4(j+s)+1}, \quad i=j+s \\
w_{4(j+m+1) \rightarrow 4(j+m+s+1)+1} \otimes w_{4(j+s)+1 \rightarrow 4(j+s)+2}, \quad i=j+3 s \\
-e_{4(j+m+s+1)+1} \otimes w_{4(j+s)+1 \rightarrow 4 j+3}, \quad i=j+5 s \\
0, \quad \text { otherwise. }
\end{array}\right.
$$

If $2 s \leqslant j<3 s$, then

$$
b_{i j}=\left\{\begin{array}{l}
-w_{4(j+m)+3 \rightarrow 4(j+m+s+1)+1} \otimes e_{4(j+s)+1}, \quad i=j+s \\
w_{4(j+m+1) \rightarrow 4(j+m+s+1)+1} \otimes w_{4(j+s)+1 \rightarrow 4(j+s)+2}, \quad i=j+3 s \\
-e_{4(j+m+s+1)+1} \otimes w_{4(j+s)+1 \rightarrow 4 j+3}, \quad i=j+5 s \\
0, \quad \text { otherwise. }
\end{array}\right.
$$

If $3 s \leqslant j<4 s$, then

$$
b_{i j}=\left\{\begin{array}{l}
-e_{4(j+m+s+1)+2} \otimes w_{4(j+s)+2 \rightarrow 4(j+1)}, \quad i=(j+s+1)_{2 s} \\
-w_{4(j+m+1) \rightarrow 4(j+m+s+1)+2} \otimes e_{4(j+s)+2}, \quad i=j+s \\
w_{4(j+m+s+1)+1 \rightarrow 4(j+m+s+1)+2} \otimes w_{4(j+s)+2 \rightarrow 4 j+3}, \quad i=j+3 s \\
0, \quad \text { otherwise. }
\end{array}\right.
$$

If $4 s \leqslant j<5 s$, then

$$
b_{i j}=\left\{\begin{array}{l}
-e_{4(j+m+s+1)+2} \otimes w_{4(j+s)+2 \rightarrow 4(j+1)}, \quad i=(j+s+1)_{2 s} \\
-w_{4(j+m+1) \rightarrow 4(j+m+s+1)+2} \otimes e_{4(j+s)+2}, \quad i=j+s \\
w_{4(j+m+s+1)+1 \rightarrow 4(j+m+s+1)+2} \otimes w_{4(j+s)+2 \rightarrow 4 j+3}, \quad i=j+3 s \\
0, \quad \text { otherwise. }
\end{array}\right.
$$

If $5 s \leqslant j<6 s$, then

$$
b_{i j}=\left\{\begin{array}{l}
\kappa^{\ell}\left(\alpha_{3(j+m+3)}\right) w_{4\left(j+m+s-f_{0}(j, 6 s-1) s+1\right)+2 \rightarrow 4(j+m+1)+3} \otimes w_{4 j+3 \rightarrow 4(j+1)}, \quad i=s+(j+1)_{s} \\
-\kappa^{\ell}\left(\alpha_{3(j+m+3)}\right) e_{4(j+m+1)+3} \otimes w_{4 j+3 \rightarrow 4\left(j+f_{0}(j, 6 s-1) s+1\right)+1}, \quad i=2 s+(j+1)_{s} ; \\
-\kappa^{\ell}\left(\alpha_{3(j+m+3)}\right) w_{4(j+m+s+1)+1 \rightarrow 4(j+m+1)+3} \otimes e_{4 j+3}, \quad i=j+s \\
-\kappa^{\ell}\left(\alpha_{3(j+m+3)}\right) w_{4(j+m+1)+1 \rightarrow 4(j+m+1)+3} \otimes e_{4 j+3}, \quad i=j+2 s \\
0, \quad \text { otherwise. }
\end{array}\right.
$$

(9) If $r_{0}=8$, then $\Omega^{8}\left(Y_{t}^{(9)}\right)$ is described with $(7 s \times 6 s)$-matrix with the following elements $b_{i j}$ : If $0 \leqslant j<s$, then

$$
b_{i j}=\left\{\begin{array}{l}
e_{4(j+m)+1} \otimes w_{4 j \rightarrow 4(j+s)+2}, \quad i=j+4 s \\
0, \quad \text { otherwise }
\end{array}\right.
$$


If $s \leqslant j<2 s$, then

$$
b_{i j}=\left\{\begin{array}{l}
-w_{4(j+m)-1 \rightarrow 4(j+m)+1} \otimes e_{4 j}, \quad i=j-s \\
e_{4(j+m)+1} \otimes w_{4 j \rightarrow 4(j+s)+2}, \quad i=j+2 s \\
0, \quad \text { otherwise. }
\end{array}\right.
$$

If $2 s \leqslant j<3 s$, then

$$
b_{i j}=\left\{\begin{array}{l}
-e_{4(j+m)+2} \otimes e_{4 j+1}, \quad i=j-s \\
0, \quad \text { otherwise }
\end{array}\right.
$$

If $3 s \leqslant j<4 s$, then

$$
b_{i j}=\left\{\begin{array}{l}
-e_{4(j+m)+2} \otimes e_{4 j+1}, \quad i=j-s \\
0, \quad \text { otherwise. }
\end{array}\right.
$$

If $4 s \leqslant j<5 s-1$, then

$$
b_{i j}=\left\{\begin{array}{l}
-\kappa^{\ell}\left(\alpha_{3(j+m+2)}\right) e_{4(j+m)+3} \otimes w_{4 j+2 \rightarrow 4(j+1)}, \quad i=(j+1)_{s} ; \\
0, \quad \text { otherwise. }
\end{array}\right.
$$

If $5 s-1 \leqslant j<4 s$, then $b_{i j}=0$.

If $4 s \leqslant j<5 s$, then

$$
b_{i j}=\left\{\begin{array}{l}
-\kappa^{\ell}\left(\alpha_{3(j+m+2)}\right) w_{4(j+m+s)+1 \rightarrow 4(j+m)+3} \otimes e_{4 j+2}, \quad i=j-s \\
0, \quad \text { otherwise. }
\end{array}\right.
$$

If $5 s \leqslant j<6 s-1$, then $b_{i j}=0$.

If $6 s-1 \leqslant j<6 s$, then

$$
b_{i j}=\left\{\begin{array}{l}
\kappa^{\ell}\left(\alpha_{3(j+m+2)}\right) e_{4(j+m)+3} \otimes w_{4 j+2 \rightarrow 4(j+1)}, \quad i=(j+1)_{s} \\
0, \quad \text { otherwise. }
\end{array}\right.
$$

If $6 s \leqslant j<5 s$, then $b_{i j}=0$.

If $5 s \leqslant j<6 s$, then

$$
b_{i j}=\left\{\begin{array}{l}
\kappa^{\ell}\left(\alpha_{3(j+m+2)}\right) w_{4(j+m+s)+1 \rightarrow 4(j+m)+3} \otimes e_{4 j+2}, \quad i=j-s \\
0, \quad \text { otherwise. }
\end{array}\right.
$$

If $6 s \leqslant j<7 s$, then

$$
b_{i j}=\left\{\begin{array}{l}
-\kappa^{\ell}\left(\alpha_{3(j+m+3)}\right) f_{2}(j, 7 s-1) e_{4(j+m+1)} \otimes e_{4 j+3}, \quad i=j-s \\
0, \quad \text { otherwise. }
\end{array}\right.
$$

(10) If $r_{0}=9$, then $\Omega^{9}\left(Y_{t}^{(9)}\right)$ is described with $(6 s \times 7 s)$-matrix with the following elements $b_{i j}$ : If $0 \leqslant j<s$, then

$$
b_{i j}=\left\{\begin{array}{l}
\kappa^{\ell}\left(\alpha_{3(j+m+2)}\right) e_{4(j+m)+3} \otimes e_{4 j}, \quad i=j \\
0, \quad \text { otherwise }
\end{array}\right.
$$


If $s \leqslant j<2 s$, then

$$
b_{i j}=\left\{\begin{array}{l}
-w_{4(j+m+1) \rightarrow 4(j+m+s+1)+2} \otimes e_{4(j+s)+1}, \quad i=j \\
-e_{4(j+m+s+1)+2} \otimes w_{4(j+s)+1 \rightarrow 4 j+3}, \quad i=j+4 s \\
0, \quad \text { otherwise }
\end{array}\right.
$$

If $2 s \leqslant j<3 s$, then

$$
b_{i j}=\left\{\begin{array}{l}
-w_{4(j+m+1) \rightarrow 4(j+m+s+1)+2} \otimes e_{4(j+s)+1}, \quad i=j ; \\
-e_{4(j+m+s+1)+2} \otimes w_{4(j+s)+1 \rightarrow 4 j+3}, \quad i=j+4 s \\
0, \quad \text { otherwise. }
\end{array}\right.
$$

If $3 s \leqslant j<4 s$, then

$$
b_{i j}=\left\{\begin{array}{l}
-e_{4(j+m+1)+1} \otimes e_{4(j+s)+2}, \quad i=j \\
0, \quad \text { otherwise }
\end{array}\right.
$$

If $4 s \leqslant j<5 s$, then

$$
b_{i j}=\left\{\begin{array}{l}
-e_{4(j+m+1)+1} \otimes e_{4(j+s)+2}, \quad i=j ; \\
0, \quad \text { otherwise. }
\end{array}\right.
$$

If $5 s \leqslant j<6 s-1$, then $b_{i j}=0$.

If $6 s-1 \leqslant j<6 s$, then

$$
b_{i j}=\left\{\begin{array}{l}
-\kappa^{\ell}\left(\alpha_{3(j+m+s-2)}\right) w_{4(j+m+1)+3 \rightarrow 4(j+m+2)} \otimes w_{4 j+3 \rightarrow 4(j+1)}, \quad i=(j+1)_{s} ; \\
0, \quad \text { otherwise. }
\end{array}\right.
$$

If $6 s \leqslant j<5 s$, then $b_{i j}=0$.

If $5 s \leqslant j<6 s$, then

$$
b_{i j}=\left\{\begin{array}{l}
\kappa^{\ell}\left(\alpha_{3(j+m+s-2)}\right) e_{4(j+m+2)} \otimes w_{4 j+3 \rightarrow 4\left(j+f_{0}(j, 6 s-1) s+1\right)+1}, \quad i=s+(j+1)_{s} \\
\kappa^{\ell}\left(\alpha_{3(j+m+s-2)}\right) w_{4(j+m+s+1)+2 \rightarrow 4(j+m+2)} \otimes e_{4 j+3}, \quad i=j \\
0, \quad \text { otherwise. }
\end{array}\right.
$$

(11) If $r_{0}=10$, then $\Omega^{10}\left(Y_{t}^{(9)}\right)$ is described with $(8 s \times 6 s)$-matrix with the following elements $b_{i j}$ :

If $0 \leqslant j<s$, then

$$
b_{i j}=\left\{\begin{array}{l}
w_{4(j+m) \rightarrow 4(j+m)+2} \otimes e_{4 j}, \quad i=j ; \\
w_{4(j+m)+1 \rightarrow 4(j+m)+2} \otimes w_{4 j \rightarrow 4(j+s)+1}, \quad i=j+2 s \\
e_{4(j+m)+2} \otimes w_{4 j \rightarrow 4(j+s)+2}, \quad i=j+4 s \\
0, \quad \text { otherwise. }
\end{array}\right.
$$


If $s \leqslant j<2 s$, then

$$
b_{i j}=\left\{\begin{array}{l}
w_{4(j+m) \rightarrow 4(j+m)+2} \otimes e_{4 j}, \quad i=j-s ; \\
-w_{4(j+m)+1 \rightarrow 4(j+m)+2} \otimes w_{4 j \rightarrow 4(j+s)+1}, \quad i=j ; \\
-e_{4(j+m)+2} \otimes w_{4 j \rightarrow 4(j+s)+2}, \quad i=j+2 s ; \\
0, \quad \text { otherwise. }
\end{array}\right.
$$

If $2 s \leqslant j<3 s$, then

$$
b_{i j}=\left\{\begin{array}{l}
\kappa^{\ell}\left(\alpha_{3(j+m+2)}\right) f_{2}(j, 3 s-1) w_{4(j+m+s)+1 \rightarrow 4(j+m)+3} \otimes e_{4 j+1}, \quad i=j-s \\
\kappa^{\ell}\left(\alpha_{3(j+m+2)}\right) f_{2}(j, 3 s-1) w_{4(j+m+s)+2 \rightarrow 4(j+m)+3} \otimes w_{4 j+1 \rightarrow 4 j+2}, \quad i=j+s \\
\kappa^{\ell}\left(\alpha_{3(j+m+2)}\right) f_{2}(j, 3 s-1) e_{4(j+m)+3} \otimes w_{4 j+1 \rightarrow 4 j+3}, \quad i=j+3 s \\
0, \quad \text { otherwise. }
\end{array}\right.
$$

If $3 s \leqslant j<4 s$, then

$$
b_{i j}=\left\{\begin{array}{l}
-\kappa^{\ell}\left(\alpha_{3(j+m+2)}\right) f_{2}(j, 4 s-1) w_{4(j+m+s)+1 \rightarrow 4(j+m)+3} \otimes e_{4 j+1}, \quad i=j-s \\
-\kappa^{\ell}\left(\alpha_{3(j+m+2)}\right) f_{2}(j, 4 s-1) w_{4(j+m+s)+2 \rightarrow 4(j+m)+3} \otimes w_{4 j+1 \rightarrow 4 j+2}, \quad i=j+s \\
\kappa^{\ell}\left(\alpha_{3(j+m+2)}\right) f_{2}(j, 4 s-1) e_{4(j+m)+3} \otimes w_{4 j+1 \rightarrow 4 j+3}, \quad i=j+2 s ; \\
0, \quad \text { otherwise. }
\end{array}\right.
$$

If $4 s \leqslant j<5 s-1$, then

$$
b_{i j}=\left\{\begin{array}{l}
\kappa^{\ell}\left(\alpha_{3(j+m+s-3)}\right) e_{4(j+m+1)} \otimes w_{4 j+2 \rightarrow 4(j+1)}, \quad i=(j+1)_{s} ; \\
0, \quad \text { otherwise. }
\end{array}\right.
$$

If $5 s-1 \leqslant j<4 s$, then $b_{i j}=0$.

If $4 s \leqslant j<5 s$, then

$$
b_{i j}=\left\{\begin{array}{l}
\kappa^{\ell}\left(\alpha_{3(j+m+s-3)}\right) w_{4(j+m+s)+2 \rightarrow 4(j+m+1)} \otimes e_{4 j+2}, \quad i=j-s \\
\kappa^{\ell}\left(\alpha_{3(j+m+s-3)}\right) w_{4(j+m)+3 \rightarrow 4(j+m+1)} \otimes w_{4 j+2 \rightarrow 4 j+3}, \quad i=j+s \\
0, \quad \text { otherwise. }
\end{array}\right.
$$

If $5 s \leqslant j<6 s-1$, then $b_{i j}=0$.

If $6 s-1 \leqslant j<6 s$, then

$$
b_{i j}=\left\{\begin{array}{l}
-\kappa^{\ell}\left(\alpha_{3(j+m+s-3)}\right) e_{4(j+m+1)} \otimes w_{4 j+2 \rightarrow 4(j+1)}, \quad i=(j+1)_{s} ; \\
0, \quad \text { otherwise. }
\end{array}\right.
$$

If $6 s \leqslant j<5 s$, then $b_{i j}=0$.

If $5 s \leqslant j<6 s$, then

$$
b_{i j}=\left\{\begin{array}{l}
-\kappa^{\ell}\left(\alpha_{3(j+m+s-3)}\right) w_{4(j+m+s)+2 \rightarrow 4(j+m+1)} \otimes e_{4 j+2}, \quad i=j-s \\
0, \quad \text { otherwise. }
\end{array}\right.
$$


If $6 s \leqslant j<7 s$, then

$$
b_{i j}=\left\{\begin{array}{l}
e_{4(j+m+1)+1} \otimes w_{4 j+3 \rightarrow 4(j+s+1)+1}, \quad i=s+(j+s+1)_{2 s} \\
w_{4(j+m)+3 \rightarrow 4(j+m+1)+1} \otimes e_{4 j+3}, \quad i=j-s \\
0, \quad \text { otherwise. }
\end{array}\right.
$$

If $7 s \leqslant j<6 s$, then $b_{i j}=0$.

If $6 s \leqslant j<7 s-1$, then

$$
b_{i j}=\left\{\begin{array}{l}
w_{4(j+m+1) \rightarrow 4(j+m+1)+1} \otimes w_{4 j+3 \rightarrow 4\left(j+s-f_{0}(j, 7 s-1) s+1\right),} \quad i=(j+1)_{s} ; \\
0, \quad \text { otherwise. }
\end{array}\right.
$$

If $7 s-1 \leqslant j<8 s-1$, then $b_{i j}=0$.

If $8 s-1 \leqslant j<8 s$, then

$$
b_{i j}=\left\{\begin{array}{l}
w_{4(j+m+1) \rightarrow 4(j+m+1)+1} \otimes w_{4 j+3 \rightarrow 4(j+1)}, \quad i=(j+1)_{s} ; \\
0, \quad \text { otherwise. }
\end{array}\right.
$$

If $8 s \leqslant j<7 s$, then $b_{i j}=0$.

If $7 s \leqslant j<8 s$, then

$$
b_{i j}=\left\{\begin{array}{l}
e_{4(j+m+1)+1} \otimes w_{4 j+3 \rightarrow 4(j+s+1)+1}, \quad i=s+(j+s+1)_{2 s} ; \\
0, \quad \text { otherwise. }
\end{array}\right.
$$

(II) Represent an arbitrary $t_{0} \in \mathbb{N}$ in the form $t_{0}=11 \ell_{0}+r_{0}$, where $0 \leqslant r_{0} \leqslant 10$. Then $\Omega^{t_{0}}\left(Y_{t}^{(9)}\right)$ is a $\Omega^{r_{0}}\left(Y_{t}^{(9)}\right)$, whose left components twisted by $\sigma^{\ell_{0}}$, and coefficients multiplied by $(-1)^{\ell_{0}}$.

Proposition 26 (Translates for the case 10). (I) Let $r_{0} \in \mathbb{N}, r_{0}<11$. Denote by

$$
\kappa_{0}=\left\{\begin{array}{l}
\kappa^{\ell}\left(\alpha_{6}\right), \quad s=1 \\
-\kappa^{\ell}\left(\gamma_{2}\right) \kappa^{\ell}\left(\alpha_{6}\right), \quad s>1
\end{array}\right.
$$

Then $r_{0}$-translates of the elements $Y_{t}^{(10)}$ are described by the following way.

(1) If $r_{0}=0$, then $\Omega^{0}\left(Y_{t}^{(10)}\right)$ is described with $(9 s \times 6 s)$-matrix with one nonzero element that is of the following form:

$$
b_{0, s}=\kappa^{\ell}\left(\alpha_{6}\right) w_{4 j \rightarrow 4(j+1)} \otimes e_{4 j} .
$$

(2) If $r_{0}=1$, then $\Omega^{1}\left(Y_{t}^{(10)}\right)$ is described with $(8 s \times 7 s)$-matrix with the following two nonzero elements:

$$
\begin{gathered}
b_{(j+s+1)_{2 s}, 2 s}=f_{2}(s, 1) \kappa_{0} w_{4(j+m+s+1)+1 \rightarrow 4(j+m+s+2)} \otimes w_{4 j+1 \rightarrow 4(j+1)} ; \\
b_{(j+s+1)_{2 s}, 3 s}=-f_{2}(s, 1) \kappa_{0} w_{4(j+m+s+1)+1 \rightarrow 4(j+m+s+2)} \otimes w_{4 j+1 \rightarrow 4(j+1)} .
\end{gathered}
$$

(3) If $r_{0}=2$, then $\Omega^{2}\left(Y_{t}^{(10)}\right)$ is described with $(9 s \times 6 s)$-matrix with one nonzero element that is of the following form:

$$
b_{(j+1)_{s}, 0}=\kappa_{0} w_{4(j+m)+3 \rightarrow 4(j+m+1)} \otimes w_{4 j \rightarrow 4(j+1)} .
$$


HOCHSCHILD COHOMOLOGY RING FOR SELF-INJECTIVE ALGEBRAS OF TREE CLASS $E_{6} . \quad$ II. 85

(4) If $r_{0}=3$, then $\Omega^{3}\left(Y_{t}^{(10)}\right)$ is described with $(8 s \times 8 s)$-matrix with the following two nonzero elements:

$$
\begin{aligned}
& b_{(j+1)_{2 s}, 0}=-\kappa^{\ell}\left(\alpha_{3(j+m+2)}\right) \kappa_{0} e_{4(j+m+1)+2} \otimes w_{4 j \rightarrow 4(j+1)} ; \\
& b_{(j+1)_{2 s}, s}=-\kappa^{\ell}\left(\alpha_{3(j+m+2)}\right) \kappa_{0} e_{4(j+m+1)+2} \otimes w_{4 j \rightarrow 4(j+1)} .
\end{aligned}
$$

(5) If $r_{0}=4$, then $\Omega^{4}\left(Y_{t}^{(10)}\right)$ is described with $(6 s \times 9 s)$-matrix with one nonzero element that is of the following form:

$$
b_{(j+1)_{s}, 0}=-f_{2}(s, 1) \kappa_{0} e_{4(j+m)+3} \otimes w_{4 j \rightarrow 4(j+1)} .
$$

(6) If $r_{0}=5$, then $\Omega^{5}\left(Y_{t}^{(10)}\right)$ is described with $(7 s \times 8 s)$-matrix with the following two nonzero elements:

$$
\begin{aligned}
b_{(j+s+1)_{2 s}, 0} & =-f_{2}(s, 1) \kappa^{\ell}\left(\gamma_{j+m}\right) \kappa_{0} w_{4(j+m+s+1)+1 \rightarrow 4(j+m+1)+3} \otimes w_{4 j \rightarrow 4(j+1)} ; \\
b_{(j+1)_{2 s}, 0} & =-f_{2}(s, 1) \kappa^{\ell}\left(\gamma_{j+m}\right) \kappa_{0} w_{4(j+m+1)+1 \rightarrow 4(j+m+1)+3} \otimes w_{4 j \rightarrow 4(j+1)} .
\end{aligned}
$$

(7) If $r_{0}=6$, then $\Omega^{6}\left(Y_{t}^{(10)}\right)$ is described with $(6 s \times 9 s)$-matrix with one nonzero element that is of the following form:

$$
b_{(j+1)_{s}, 0}=-f_{2}(s, 1) \kappa_{0} e_{4(j+m+1)} \otimes w_{4 j \rightarrow 4(j+1)} .
$$

(8) If $r_{0}=7$, then $\Omega^{7}\left(Y_{t}^{(10)}\right)$ is described with $(6 s \times 8 s)$-matrix with one nonzero element that is of the following form:

$$
b_{(j+1)_{s}, 0}=\kappa_{1} \kappa_{0} w_{4(j+m+s f(j, s-1)+1)+2 \rightarrow 4(j+m+2)} \otimes w_{4 j \rightarrow 4(j+1)},
$$

where

$$
\kappa_{1}=\left\{\begin{array}{l}
-\kappa^{\ell}\left(\gamma_{m}\right), \quad s=1 \\
\kappa^{\ell}\left(\gamma_{m}\right) \kappa^{\ell}\left(\gamma_{m+1}\right) \kappa^{\ell}\left(\gamma_{m+2}\right), \quad s>1 .
\end{array}\right.
$$

(9) If $r_{0}=8$, then $\Omega^{8}\left(Y_{t}^{(10)}\right)$ is described with $(7 s \times 6 s)$-matrix with one nonzero element that is of the following form:

$$
b_{(j+1)_{s}, s(1-f(s, 1))}=\kappa_{1} \kappa_{0} w_{4(j+m)+3 \rightarrow 4(j+m+s+1)+1} \otimes w_{4 j \rightarrow 4(j+1)},
$$

where

$$
\kappa_{1}=\left\{\begin{array}{l}
-\kappa^{\ell}\left(\alpha_{3(m+4)}\right), \quad s=1 ; \\
\kappa^{\ell}\left(\gamma_{m-1}\right) \kappa^{\ell}\left(\gamma_{m}\right) \kappa^{\ell}\left(\gamma_{m+1}\right) \kappa^{\ell+1}\left(\alpha_{0}\right), \quad s>1 .
\end{array}\right.
$$

(10) If $r_{0}=9$ and $s=1$, then $\Omega^{9}\left(Y_{t}^{(10)}\right)$ is described with $(6 s \times 7 s)$-matrix with one nonzero element that is of the following form:

$$
b_{(j+1)_{s}, 0}=-\kappa^{\ell}\left(\gamma_{j+m}\right) \kappa_{0} e_{4(j+m+1)+3} \otimes w_{4 j \rightarrow 4(j+1)} .
$$

(11) If $r_{0}=9$ and $s>1$, then $\Omega^{9}\left(Y_{t}^{(10)}\right)$ is described with $(6 s \times 7 s)$-matrix with the following two nonzero elements:

$$
\begin{aligned}
& b_{5 s+j, 0}=-\kappa_{1} \kappa_{0} w_{4(j+m+1)+2 \rightarrow 4(j+m+1)+3} \otimes w_{4 j \rightarrow 4 j+3} ; \\
& b_{6 s+j, 0}=\kappa_{1} \kappa_{0} w_{4(j+m+s+1)+2 \rightarrow 4(j+m+1)+3} \otimes w_{4 j \rightarrow 4 j+3},
\end{aligned}
$$

where $\kappa_{1}=-\kappa^{\ell}\left(\gamma_{m-1}\right) \kappa^{\ell}\left(\gamma_{m}\right) \kappa^{\ell}\left(\gamma_{m+1}\right)$. 
(12) If $r_{0}=10$ and $s=1$, then $\Omega^{10}\left(Y_{t}^{(10)}\right)$ is described with $(8 s \times 6 s)$-matrix with the following two nonzero elements:

$$
\begin{gathered}
b_{(j+1)_{s}, 0}=-\kappa^{\ell}\left(\alpha_{3(j+m+3)}\right) \kappa_{0} w_{4(j+m+1) \rightarrow 4(j+m+s+1)+2} \otimes w_{4 j \rightarrow 4(j+1)} ; \\
b_{(j+1)_{s}, s}=\kappa^{\ell}\left(\alpha_{3(j+m+3)}\right) \kappa_{0} w_{4(j+m+1) \rightarrow 4(j+m+s+1)+2} \otimes w_{4 j \rightarrow 4(j+1)} .
\end{gathered}
$$

(13) If $r_{0}=10$ and $s>1$, then $\Omega^{10}\left(Y_{t}^{(10)}\right)$ is described with $(8 s \times 6 s)$-matrix with the following nonzero elements:

$$
\begin{gathered}
b_{(j+1)_{s}, 0}=\kappa_{1} \kappa_{0} w_{4(j+m+1) \rightarrow 4(j+m+s+1)+2} \otimes w_{4 j \rightarrow 4(j+1)} ; \\
b_{5 s+j, 0}=-\kappa_{1} \kappa_{0} w_{4(j+m+1)-1 \rightarrow 4(j+m+s+1)+2} \otimes w_{4 j \rightarrow 4(j+1)-1} ; \\
b_{(j+1)_{s}, s}=\kappa_{1} \kappa_{0} w_{4(j+m+1) \rightarrow 4(j+m+s+1)+2} \otimes w_{4 j \rightarrow 4(j+1)} ; \\
b_{4 s+j, s}=-\kappa_{1} \kappa_{0} w_{4(j+m+1)-1 \rightarrow 4(j+m+s+1)+2} \otimes w_{4 j \rightarrow 4(j+1)-1} ; \\
b_{5 s+(j+1)_{s}, 7 s-1+s f\left((j)_{s}, 0\right)}=\kappa_{1} \kappa_{0} w_{4(j+m+s+1)+3 \rightarrow 4(j+m+s+1)+5} \otimes w_{4 j+3 \rightarrow 4(j+1)+3},
\end{gathered}
$$

where $\kappa_{1}=\kappa^{\ell}\left(\gamma_{m-2}\right) \kappa^{\ell}\left(\gamma_{m-1}\right) \kappa^{\ell}\left(\gamma_{m}\right) \kappa^{\ell}\left(\gamma_{m+1}\right) \kappa^{\ell+1}\left(\alpha_{3}\right)$.

(II) Represent an arbitrary $t_{0} \in \mathbb{N}$ in the form $t_{0}=11 \ell_{0}+r_{0}$, where $0 \leqslant r_{0} \leqslant 10$. Then $\Omega^{t_{0}}\left(Y_{t}^{(10)}\right)$ is a $\Omega^{r_{0}}\left(Y_{t}^{(10)}\right)$, whose left components twisted by $\sigma^{\ell_{0}}$, and coefficients multiplied by $(-1)^{\ell_{0}}$.

Proposition 27 (Translates for the case 11). (I) Let $r_{0} \in \mathbb{N}, r_{0}<11$. $r_{0}$-translates of the elements $Y_{t}^{(11)}$ are described by the following way.

(1) If $r_{0}=0$, then $\Omega^{0}\left(Y_{t}^{(11)}\right)$ is described with $(8 s \times 6 s)$-matrix with the following elements $b_{i j}$ : If $0 \leqslant j<2 s$, then

$$
b_{i j}=\left\{\begin{array}{l}
w_{4(j+m) \rightarrow 4(j+m)+1} \otimes e_{4 j}, \quad i=(j)_{s} \\
0, \quad \text { otherwise }
\end{array}\right.
$$

If $2 s \leqslant j<4 s$, then

$$
b_{i j}=\left\{\begin{array}{l}
\kappa^{\ell}\left(\alpha_{3(j+m+2+1)}\right) w_{4(j+m)+1 \rightarrow 4(j+m+1)} \otimes e_{4 j+1}, \quad i=j-s \\
0, \quad \text { otherwise }
\end{array}\right.
$$

If $4 s \leqslant j<6 s$, then

$$
b_{i j}=\left\{\begin{array}{l}
-\kappa^{\ell}\left(\alpha_{3(j+m+2)}\right) w_{4(j+m)+2 \rightarrow 4(j+m)+3} \otimes e_{4 j+2}, \quad i=j-s \\
0, \quad \text { otherwise. }
\end{array}\right.
$$

If $6 s \leqslant j<8 s$, then

$$
b_{i j}=\left\{\begin{array}{l}
f_{1}(j, 7 s) w_{4(j+m)+3 \rightarrow 4(j+m+1)+2} \otimes e_{4 j+3}, \quad i=5 s+(j)_{s} \\
0, \quad \text { otherwise. }
\end{array}\right.
$$

(2) If $r_{0}=1$, then $\Omega^{1}\left(Y_{t}^{(11)}\right)$ is described with $(9 s \times 7 s)$-matrix with the following elements $b_{i j}$ : 
If $0 \leqslant j<s$, then

$$
b_{i j}=\left\{\begin{array}{l}
\kappa_{1} w_{4(j+m)+1 \rightarrow 4(j+m+1)} \otimes e_{4 j}, \quad i=j \\
\kappa_{1} w_{4(j+m+s)+1 \rightarrow 4(j+m+1)} \otimes e_{4 j}, \quad i=j+s \\
-\kappa_{1} w_{4(j+m)+2 \rightarrow 4(j+m+1)} \otimes w_{4 j \rightarrow 4 j+1}, \quad i=j+2 s \\
-\kappa_{1} w_{4(j+m+s)+2 \rightarrow 4(j+m+1)} \otimes w_{4 j \rightarrow 4(j+s)+1}, \quad i=j+3 s \\
0, \quad \text { otherwise, }
\end{array}\right.
$$

where $\kappa_{1}=\kappa^{\ell}\left(\alpha_{3(j+m+3)}\right)$.

If $s \leqslant j<2 s$, then

$$
b_{i j}=\left\{\begin{array}{l}
w_{4(j+m+s)+2 \rightarrow 4(j+m+s+1)+1} \otimes e_{4(j+s)+1}, \quad i=j+s \\
w_{4(j+m)+3 \rightarrow 4(j+m+s+1)+1} \otimes w_{4(j+s)+1 \rightarrow 4(j+s)+2}, \quad i=j+3 s \\
w_{4(j+m+1) \rightarrow 4(j+m+s+1)+1} \otimes w_{4(j+s)+1 \rightarrow 4 j+3}, \quad i=j+5 s \\
0, \quad \text { otherwise }
\end{array}\right.
$$

If $2 s \leqslant j<3 s$, then

$$
b_{i j}=\left\{\begin{array}{l}
w_{4(j+m+s+1)+1 \rightarrow 4(j+m+s+1)+2} \otimes w_{4 j+1 \rightarrow 4(j+1)}, \quad i=(j+s+1)_{2 s} \\
-w_{4(j+m)+3 \rightarrow 4(j+m+s+1)+2} \otimes w_{4 j+1 \rightarrow 4 j+2}, \quad i=j+2 s \\
0, \quad \text { otherwise. }
\end{array}\right.
$$

If $3 s \leqslant j<4 s$, then

$$
b_{i j}= \begin{cases}w_{4(j+m)+2 \rightarrow 4(j+m+1)+1} \otimes e_{4 j+1}, \quad i=j ; \\ w_{4(j+m)+3 \rightarrow 4(j+m+1)+1} \otimes w_{4 j+1 \rightarrow 4 j+2}, & i=j+2 s \\ w_{4(j+m+1) \rightarrow 4(j+m+1)+1} \otimes w_{4 j+1 \rightarrow 4 j+3}, & i=j+3 s \\ 0, \quad \text { otherwise. } & \end{cases}
$$

If $4 s \leqslant j<5 s$, then

$$
b_{i j}=\left\{\begin{array}{l}
w_{4(j+m+1)+1 \rightarrow 4(j+m+1)+2} \otimes w_{4(j+s)+1 \rightarrow 4(j+1),} \quad i=(j+1)_{2 s} \\
-w_{4(j+m)+3 \rightarrow 4(j+m+1)+2} \otimes w_{4(j+s)+1 \rightarrow 4(j+s)+2}, \quad i=j+s \\
0, \quad \text { otherwise }
\end{array}\right.
$$

If $5 s \leqslant j<7 s$, then

$$
b_{i j}=\left\{\begin{array}{l}
-w_{4(j+m)+3 \rightarrow 4(j+m+s+1)+2} \otimes e_{4(j+s)+2}, \quad i=j-s \\
0, \quad \text { otherwise. }
\end{array}\right.
$$


If $7 s \leqslant j<8 s$, then

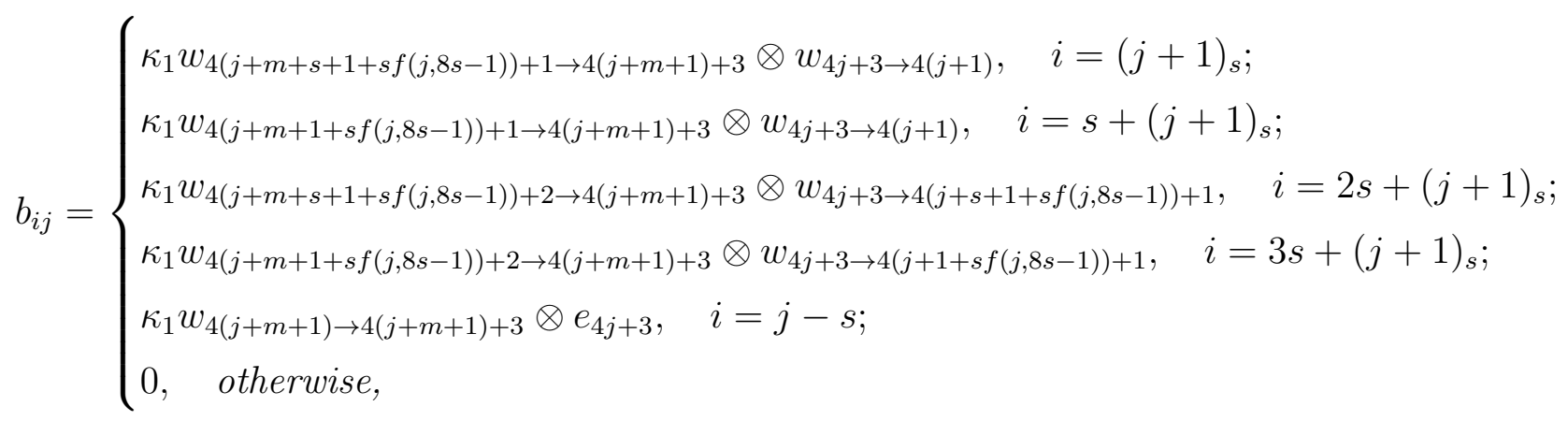

where $\kappa_{1}=-\kappa^{\ell}\left(\alpha_{3(j+m+3)}\right)$.

If $8 s \leqslant j<9 s$, then

$$
b_{i j}=\left\{\begin{array}{l}
\kappa_{1} w_{4(j+m+s+1)+1 \rightarrow 4(j+m+2)} \otimes w_{4 j+3 \rightarrow 4(j+1)}, \quad i=(j+s+1)_{2 s} \\
\kappa_{1} w_{4(j+m+1) \rightarrow 4(j+m+2)} \otimes e_{4 j+3}, \quad i=j-2 s \\
0, \quad \text { otherwise }
\end{array}\right.
$$

where $\kappa_{1}=\kappa^{\ell}\left(\alpha_{3(j+m+4)}\right)$.

(3) If $r_{0}=2$, then $\Omega^{2}\left(Y_{t}^{(11)}\right)$ is described with $(8 s \times 6 s)$-matrix with the following elements $b_{i j}$ : If $0 \leqslant j<2 s$, then

$$
b_{i j}=\left\{\begin{array}{l}
-f_{1}(j, s) w_{4(j+m)-1 \rightarrow 4(j+m)+2} \otimes e_{4 j}, \quad i=(j)_{s} \\
-e_{4(j+m)+2} \otimes w_{4 j \rightarrow 4 j+1}, \quad i=j+s \\
0, \quad \text { otherwise. }
\end{array}\right.
$$

If $2 s \leqslant j<4 s$, then

$$
b_{i j}=\left\{\begin{array}{l}
\kappa^{\ell+1}\left(\alpha_{3(j+m+2)}\right) e_{4(j+m)+3} \otimes w_{4 j+1 \rightarrow 4(j+1)}, \quad i=(j+1)_{s} \\
0, \quad \text { otherwise. }
\end{array}\right.
$$

If $4 s \leqslant j<6 s$, then

$$
b_{i j}=\left\{\begin{array}{l}
-\kappa^{\ell}\left(\alpha_{3(j+m+3)}\right) w_{4(j+m+s)+1 \rightarrow 4(j+m+1)} \otimes e_{4 j+2}, \quad i=j-s \\
0, \quad \text { otherwise. }
\end{array}\right.
$$

If $6 s \leqslant j<8 s$, then

$$
b_{i j}=\left\{\begin{array}{l}
-f_{2}\left((j)_{s}, s-1\right) f_{1}(j, 7 s) w_{4(j+m)+3 \rightarrow 4(j+m+1)+1} \otimes w_{4 j+3 \rightarrow 4(j+1)}, \quad i=(j+1)_{s} \\
e_{4(j+m+1)+1} \otimes w_{4 j+3 \rightarrow 4(j+s+1)+2}, \quad i=3 s+(j+s+1)_{2 s} \\
0, \quad \text { otherwise. }
\end{array}\right.
$$

(4) If $r_{0}=3$, then $\Omega^{3}\left(Y_{t}^{(11)}\right)$ is described with $(6 s \times 8 s)$-matrix with the following elements $b_{i j}$ : 
If $0 \leqslant j<s$, then

$$
b_{i j}=\left\{\begin{array}{l}
-\kappa_{1} w_{4(j+m+s)+2 \rightarrow 4(j+m)+3} \otimes e_{4 j}, \quad i=j+s \\
\kappa_{1} e_{4(j+m)+3} \otimes w_{4 j \rightarrow 4 j+1}, \quad i=j+2 s \\
0, \quad \text { otherwise }
\end{array}\right.
$$

where $\kappa_{1}=-\kappa^{\ell}\left(\alpha_{3(j+m+2)}\right)$.

If $s \leqslant j<3 s$, then

$$
b_{i j}=\left\{\begin{array}{l}
f_{2}\left((j)_{s}, s-1\right) f_{1}(j, 2 s) e_{4(j+m+s+1)+2} \otimes w_{4(j+s)+1 \rightarrow 4(j+1)}, \quad i=(j+s+1)_{2 s} ; \\
0, \quad \text { otherwise. }
\end{array}\right.
$$

If $3 s \leqslant j<4 s$, then

$$
b_{i j}=\left\{\begin{array}{l}
-w_{4(j+m+1) \rightarrow 4(j+m+1)+1} \otimes e_{4(j+s)+2}, \quad i=j+s \\
-e_{4(j+m+1)+1} \otimes w_{4(j+s)+2 \rightarrow 4 j+3}, \quad i=j+4 s \\
0, \quad \text { otherwise. }
\end{array}\right.
$$

If $4 s \leqslant j<5 s$, then

$$
b_{i j}=\left\{\begin{array}{l}
-w_{4(j+m+1) \rightarrow 4(j+m+1)+1} \otimes e_{4(j+s)+2}, \quad i=j+s \\
-e_{4(j+m+1)+1} \otimes w_{4(j+s)+2 \rightarrow 4 j+3}, \quad i=j+2 s \\
0, \quad \text { otherwise. }
\end{array}\right.
$$

If $5 s \leqslant j<6 s$, then

$$
b_{i j}=\left\{\begin{array}{l}
\kappa_{1} w_{4(j+m+s+1+s f(j, 6 s-1))+2 \rightarrow 4(j+m+2)} \otimes w_{4 j+3 \rightarrow 4(j+1)}, \quad i=(j+1)_{s} ; \\
\kappa_{1} w_{4(j+m+1+s f(j, 6 s-1))+2 \rightarrow 4(j+m+2)} \otimes w_{4 j+3 \rightarrow 4(j+1)}, \quad i=s+(j+1)_{s} ; \\
\kappa_{1} w_{4(j+m+1)+3 \rightarrow 4(j+m+2)} \otimes w_{4 j+3 \rightarrow 4(j+s+1+s f(j, 6 s-1))+1}, \quad i=2 s+(j+1)_{s} ; \\
0, \quad \text { otherwise, }
\end{array}\right.
$$

where $\kappa_{1}=-\kappa^{\ell}\left(\alpha_{3(j+m+4)}\right)$.

(5) If $r_{0}=4$, then $\Omega^{4}\left(Y_{t}^{(11)}\right)$ is described with $(7 s \times 9 s)$-matrix with the following elements $b_{i j}$ : If $0 \leqslant j<s$, then

$$
b_{i j}=\left\{\begin{array}{l}
\kappa_{1} w_{4(j+m)-1 \rightarrow 4(j+m)+3} \otimes e_{4 j}, \quad i=j ; \\
-\kappa_{1} e_{4(j+m)+3} \otimes w_{4 j \rightarrow 4(j+1)}, \quad i=(j+1)_{s}, \quad j<s-1 ; \\
-\kappa_{1} w_{4(j+m)+1 \rightarrow 4(j+m)+3} \otimes w_{4 j \rightarrow 4(j+s)+1}, \quad i=j+3 s \\
\kappa_{1} w_{4(j+m+s)+2 \rightarrow 4(j+m)+3} \otimes w_{4 j \rightarrow 4 j+2}, \quad i=j+5 s ; \\
0, \quad \text { otherwise, }
\end{array}\right.
$$

where $\kappa_{1}=-\kappa^{\ell}\left(\alpha_{3(j+m+2)}\right)$. 
If $s \leqslant j<3 s$, then

$$
b_{i j}=\left\{\begin{array}{l}
\kappa_{1} f_{2}\left((j)_{s}, s-1\right) f_{1}(j, 2 s) e_{4(j+m+1)} \otimes w_{4(j+s)+1 \rightarrow 4(j+1)}, \quad i=s+(j+1)_{s} \\
-\kappa_{1} w_{4(j+m)+1 \rightarrow 4(j+m+1)} \otimes e_{4(j+s)+1}, \quad i=j+s \\
0, \quad \text { otherwise, }
\end{array}\right.
$$

where $\kappa_{1}=-\kappa^{\ell}\left(\alpha_{3(j+m+3)}\right)$.

If $3 s \leqslant j<4 s$, then

$$
b_{i j}=\left\{\begin{array}{l}
w_{4(j+m)+3 \rightarrow 4(j+m+1)+1} \otimes w_{4(j+s)+2 \rightarrow 4(j+1)}, \quad i=(j+1)_{s}, \quad j<4 s-1 \\
-f_{2}\left((j)_{s}, s-1\right) w_{4(j+m+1) \rightarrow 4(j+m+1)+1} \otimes w_{4(j+s)+2 \rightarrow 4(j+1)}, \quad i=s+(j+1)_{s} ; \\
-w_{4(j+m)+2 \rightarrow 4(j+m+1)+1} \otimes e_{4(j+s)+2}, \quad i=j+2 s \\
0, \quad \text { otherwise. }
\end{array}\right.
$$

If $4 s \leqslant j<5 s$, then

$$
b_{i j}=\left\{\begin{array}{l}
w_{4(j+m)+3 \rightarrow 4(j+m+1)+1} \otimes w_{4(j+s)+2 \rightarrow 4(j+1)}, \quad i=(j+1)_{s}, \quad j=5 s-1 \\
f_{2}(j, 5 s-1) w_{4(j+m+1) \rightarrow 4(j+m+1)+1} \otimes w_{4(j+s)+2 \rightarrow 4(j+1)}, \quad i=s+(j+1)_{s} \\
-w_{4(j+m)+2 \rightarrow 4(j+m+1)+1} \otimes e_{4(j+s)+2}, \quad i=j+3 s \\
0, \quad \text { otherwise. }
\end{array}\right.
$$

If $5 s \leqslant j<6 s$, then

$$
b_{i j}=\left\{\begin{array}{l}
w_{4(j+m+s+1)+1 \rightarrow 4(j+m+s+1)+2} \otimes w_{4 j+3 \rightarrow 4(j+1)+1}, \quad i=2 s+(j+1)_{2 s} \\
-w_{4(j+m)+3 \rightarrow 4(j+m+s+1)+2} \otimes w_{4 j+3 \rightarrow 4(j+1)}, \quad i=(j+1)_{s}, \quad j<6 s-1 \\
e_{4(j+m+s+1)+2} \otimes w_{4 j+3 \rightarrow 4(j+1)+2}, \quad i=7 s+(j+1)_{s}, \quad j<6 s-1 \\
e_{4(j+m+s+1)+2} \otimes w_{4 j+3 \rightarrow 4(j+1)+2}, \quad i=5 s+(j+1)_{s}, \quad j=6 s-1 \\
0, \quad \text { otherwise. }
\end{array}\right.
$$

If $6 s \leqslant j<7 s$, then

$$
b_{i j}=\left\{\begin{array}{l}
w_{4(j+m+s+1)+1 \rightarrow 4(j+m+s+1)+2} \otimes w_{4 j+3 \rightarrow 4(j+1)+1}, \quad i=2 s+(j+1)_{2 s} \\
e_{4(j+m+s+1)+2} \otimes w_{4 j+3 \rightarrow 4(j+1)+2}, \quad i=5 s+(j+1)_{s}, \quad j<7 s-1 \\
-w_{4(j+m)+3 \rightarrow 4(j+m+s+1)+2} \otimes w_{4 j+3 \rightarrow 4(j+1)}, \quad i=(j+1)_{s}, \quad j=7 s-1 \\
e_{4(j+m+s+1)+2} \otimes w_{4 j+3 \rightarrow 4(j+1)+2}, \quad i=7 s+(j+1)_{s}, \quad j=7 s-1 \\
0, \quad \text { otherwise. }
\end{array}\right.
$$

(6) If $r_{0}=5$, then $\Omega^{5}\left(Y_{t}^{(11)}\right)$ is described with $(6 s \times 8 s)$-matrix with the following elements $b_{i j}$ : If $0 \leqslant j<s$, then

$$
b_{i j}=\left\{\begin{array}{l}
\kappa^{\ell}\left(\alpha_{3(j+m+3)}\right) e_{4(j+m+1)} \otimes w_{4 j \rightarrow 4 j+1}, \quad i=j+2 s \\
0, \quad \text { otherwise. }
\end{array}\right.
$$


HOCHSCHILD COHOMOLOGY RING FOR SELF-INJECTIVE ALGEBRAS OF TREE CLASS E. II. 91 If $s \leqslant j<3 s$, then

$$
b_{i j}=\left\{\begin{array}{l}
w_{4(j+m+1) \rightarrow 4(j+m+1)+1} \otimes e_{4(j+s)+1}, \quad i=j+s \\
0, \quad \text { otherwise. }
\end{array}\right.
$$

If $3 s \leqslant j<5 s$, then

$$
b_{i j}=\left\{\begin{array}{l}
w_{4(j+m+1)+1 \rightarrow 4(j+m+1)+2} \otimes w_{4(j+s)+2 \rightarrow 4(j+1)}, \quad i=(j+1)_{2 s} ; \\
w_{4(j+m)+3 \rightarrow 4(j+m+1)+2} \otimes e_{4(j+s)+2}, \quad i=j+s \\
f_{1}(j, 4 s) e_{4(j+m+1)+2} \otimes w_{4(j+s)+2 \rightarrow 4 j+3}, \quad i=6 s+(j)_{2 s} \\
0, \quad \text { otherwise. }
\end{array}\right.
$$

If $5 s \leqslant j<6 s$, then

$$
b_{i j}=\left\{\begin{array}{l}
-\kappa_{1} w_{4(j+m+s+1+s f(j, 6 s-1))+1 \rightarrow 4(j+m+1)+3} \otimes w_{4 j+3 \rightarrow 4(j+1)}, \quad i=(j+1)_{s} ; \\
-\kappa_{1} w_{4(j+m+1+s f(j, 6 s-1))+1 \rightarrow 4(j+m+1)+3} \otimes w_{4 j+3 \rightarrow 4(j+1)}, \quad i=s+(j+1)_{s} ; \\
\kappa_{1} e_{4(j+m+1)+3} \otimes w_{4 j+3 \rightarrow 4(j+s+1+s f(j, 6 s-1))+2}, \quad i=4 s+(j+1)_{s} ; \\
\kappa_{1} e_{4(j+m+1)+3} \otimes w_{4 j+3 \rightarrow 4(j+1+s f(j, 6 s-1))+2}, \quad i=5 s+(j+1)_{s} ; \\
0, \quad \text { otherwise, }
\end{array}\right.
$$

where $\kappa_{1}=\kappa^{\ell}\left(\alpha_{3(j+m+3)}\right) f_{2}\left((j)_{s}, s-1\right)$.

(7) If $r_{0}=6$, then $\Omega^{6}\left(Y_{t}^{(11)}\right)$ is described with $(6 s \times 9 s)$-matrix with the following elements $b_{i j}$ : If $0 \leqslant j<s$, then

$$
b_{i j}=\left\{\begin{array}{l}
\kappa_{1} w_{4(j+m)+1 \rightarrow 4(j+m+1)} \otimes w_{4 j \rightarrow 4 j+1}, \quad i=j+s \\
-\kappa_{1} w_{4(j+m+s)+2 \rightarrow 4(j+m+1)} \otimes w_{4 j \rightarrow 4 j+1}, \quad i=j+2 s \\
\kappa_{1} w_{4(j+m)+2 \rightarrow 4(j+m+1)} \otimes w_{4 j \rightarrow 4(j+s)+1}, \quad i=j+4 s \\
\kappa_{1} e_{4(j+m+1)} \otimes w_{4 j \rightarrow 4(j+1)}, \quad i=(j+1)_{s}, \quad j=s-1 ; \\
0, \quad \text { otherwise, }
\end{array}\right.
$$

where $\kappa_{1}=\kappa^{\ell}\left(\alpha_{3(j+m+3)}\right)$.

If $s \leqslant j<3 s$, then

$$
b_{i j}=\left\{\begin{array}{l}
w_{4(j+m)+2 \rightarrow 4(j+m+1)+1} \otimes e_{4(j+s)+1}, \quad i=j+2 s-s f_{0}(j, 2 s) ; \\
-w_{4(j+m+1) \rightarrow 4(j+m+1)+1} \otimes w_{4(j+s)+1 \rightarrow 4(j+1)}, \quad i=(j+1)_{s}, \quad 2 s-1 \leqslant j<3 s-1 ; \\
0, \quad \text { otherwise. }
\end{array}\right.
$$

If $3 s \leqslant j<5 s$, then

$$
b_{i j}= \begin{cases}-w_{4(j+m)+3 \rightarrow 4(j+m+1)+2} \otimes w_{4(j+s)+2 \rightarrow 4 j+3}, & i=7 s+(j)_{s} ; \\ w_{4(j+m+1) \rightarrow 4(j+m+1)+2} \otimes w_{4(j+s)+2 \rightarrow 4(j+1)}, & i=(j+1)_{s}, \quad 4 s-1 \leqslant j<5 s-1 ; \\ 0, \quad \text { otherwise. } & \end{cases}
$$


If $5 s \leqslant j<6 s$, then

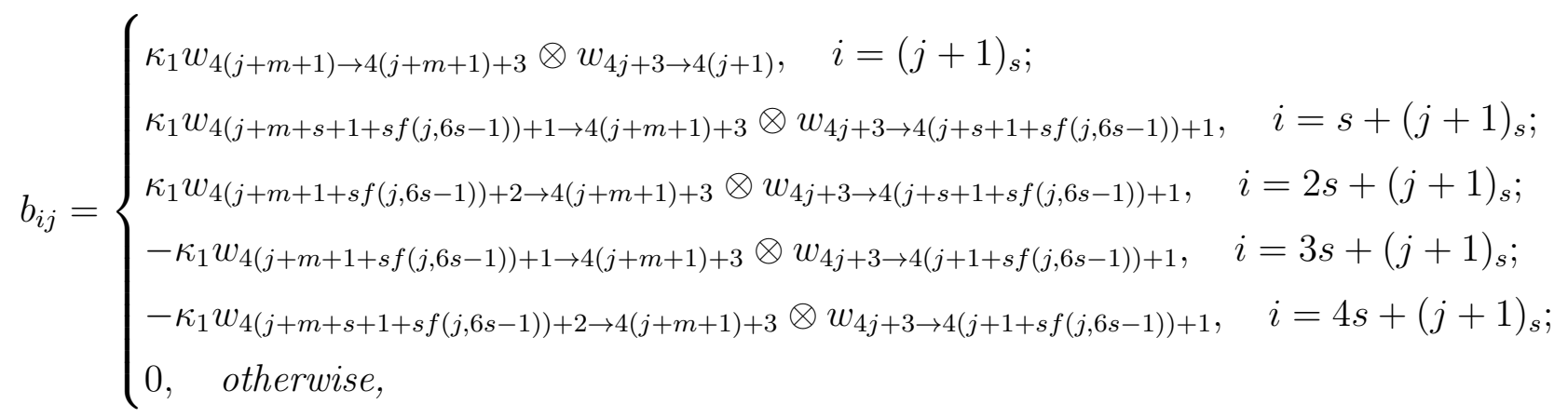

where $\kappa_{1}=-\kappa^{\ell}\left(\alpha_{3(j+m+3)}\right) f_{2}(j, 6 s-1)$.

(8) If $r_{0}=7$, then $\Omega^{7}\left(Y_{t}^{(11)}\right)$ is described with $(7 s \times 8 s)$-matrix with the following elements $b_{i j}$ : If $s \leqslant j<2 s$, then

$$
b_{i j}=\left\{\begin{array}{l}
w_{4(j+m)+3 \rightarrow 4(j+m+s+1)+1} \otimes w_{4 j \rightarrow 4(j+s)+1}, \quad i=j+s \\
w_{4(j+m+1) \rightarrow 4(j+m+s+1)+1} \otimes w_{4 j \rightarrow 4(j+s)+2}, \quad i=j+3 s \\
w_{4(j+m+1) \rightarrow 4(j+m+s+1)+1} \otimes w_{4 j \rightarrow 4 j+2}, \quad i=j+4 s \\
0, \quad \text { otherwise. }
\end{array}\right.
$$

If $2 s \leqslant j<4 s$, then

$$
b_{i j}=\left\{\begin{array}{l}
w_{4(j+m)+3 \rightarrow 4(j+m+s+1)+2} \otimes e_{4 j+1}, \quad i=j \\
0, \quad \text { otherwise. }
\end{array}\right.
$$

If $4 s \leqslant j<6 s$, then

$$
b_{i j}=\left\{\begin{array}{l}
\kappa_{1} w_{4(j+m+s+1)+2 \rightarrow 4(j+m+1)+3} \otimes w_{4 j+2 \rightarrow 4(j+1)}, \quad i=(j+s+1)_{2 s} ; \\
\kappa_{1} w_{4(j+m+1) \rightarrow 4(j+m+1)+3} \otimes e_{4 j+2}, \quad i=j ; \\
-\kappa_{1} w_{4(j+m+1)+1 \rightarrow 4(j+m+1)+3} \otimes w_{4 j+2 \rightarrow 4 j+3}, \quad i=j+2 s ; \\
0, \quad \text { otherwise, }
\end{array}\right.
$$

where $\kappa_{1}=\kappa^{\ell}\left(\alpha_{3(j+m+3)}\right) f_{1}(j, 5 s)$.

If $6 s \leqslant j<7 s$, then

$$
b_{i j}=\left\{\begin{array}{l}
\kappa_{1} w_{4(j+m+s+1+s f(j, 7 s-1))+2 \rightarrow 4(j+m+2)} \otimes w_{4 j+3 \rightarrow 4(j+1)}, \quad i=s+(j+1)_{s} ; \\
-\kappa_{1} w_{4(j+m+1)+3 \rightarrow 4(j+m+2)} \otimes w_{4 j+3 \rightarrow 4(j+1+s f(j, 7 s-1))+1}, \quad i=2 s+(j+1)_{s} ; \\
-\kappa_{1} e_{4(j+m+2)} \otimes w_{4 j+3 \rightarrow 4(j+1+s f(j, 7 s-1))+2}, \quad i=4 s+(j+1)_{s} ; \\
-\kappa_{1} e_{4(j+m+2)} \otimes w_{4 j+3 \rightarrow 4(j+s+1+s f(j, 7 s-1))+2}, \quad i=5 s+(j+1)_{s} ; \\
0, \quad \text { otherwise, }
\end{array}\right.
$$

where $\kappa_{1}=-\kappa^{\ell}\left(\alpha_{3(j+m+4)}\right)$.

(9) If $r_{0}=8$, then $\Omega^{8}\left(Y_{t}^{(11)}\right)$ is described with $(6 s \times 6 s)$-matrix with the following elements $b_{i j}$ : 
If $0 \leqslant j<s$, then

$$
b_{i j}=\left\{\begin{array}{l}
-\kappa_{1} w_{4(j+m)-1 \rightarrow 4(j+m)+3} \otimes e_{4 j}, \quad i=j ; \\
\kappa_{1} f_{2}(j, s-1) e_{4(j+m)+3} \otimes w_{4 j \rightarrow 4(j+1)}, \quad i=(j+1)_{s} ; \\
\kappa_{1} w_{4(j+m)+2 \rightarrow 4(j+m)+3} \otimes w_{4 j \rightarrow 4 j+1}, \quad i=j+s ; \\
-\kappa_{1} w_{4(j+m+s)+1 \rightarrow 4(j+m)+3} \otimes w_{4 j \rightarrow 4 j+2}, \quad i=j+3 s \\
0, \quad \text { otherwise, }
\end{array}\right.
$$

where $\kappa_{1}=\kappa^{\ell}\left(\alpha_{3(j+m+2)}\right)$.

If $s \leqslant j<3 s$, then

$$
b_{i j}=\left\{\begin{array}{l}
-w_{4(j+m)+3 \rightarrow 4(j+m+1)+2} \otimes w_{4(j+s)+1 \rightarrow 4(j+1)}, \quad i=(j+1)_{s} ; \\
0, \quad \text { otherwise. }
\end{array}\right.
$$

If $3 s \leqslant j<5 s$, then

$$
b_{i j}=\left\{\begin{array}{l}
w_{4(j+m+1) \rightarrow 4(j+m+s+1)+1} \otimes w_{4(j+s)+2 \rightarrow 4 j+3}, \quad i=5 s+(j)_{s} ; \\
0, \quad \text { otherwise. }
\end{array}\right.
$$

If $5 s \leqslant j<6 s$, then

$$
b_{i j}=\left\{\begin{array}{l}
\kappa_{1} w_{4(j+m+s+1+s f(j, 6 s-1))+2 \rightarrow 4(j+m+2)} \otimes w_{4 j+3 \rightarrow 4(j+s+1+s f(j, 6 s-1))+1,} \quad i=s+(j+1)_{s} ; \\
\kappa_{1} w_{4(j+m+1+s f(j, 6 s-1))+2 \rightarrow 4(j+m+2)} \otimes w_{4 j+3 \rightarrow 4(j+1+s f(j, 6 s-1))+1}, \quad i=2 s+(j+1)_{s} ; \\
\kappa_{1} w_{4(j+m+1+s f(j, 6 s-1))+1 \rightarrow 4(j+m+2)} \otimes w_{4 j+3 \rightarrow 4(j+s+1+s f(j, 6 s-1))+2,} \quad i=3 s+(j+1)_{s} ; \\
0, \quad \text { otherwise, }
\end{array}\right.
$$

where $\kappa_{1}=\kappa^{\ell}\left(\alpha_{3(j+m-1)}\right) \kappa^{\ell+1}\left(\gamma_{j+m-2}\right)$.

(10) If $r_{0}=9$, then $\Omega^{9}\left(Y_{t}^{(11)}\right)$ is described with $(8 s \times 7 s)$-matrix with the following elements $b_{i j}$ :

If $0 \leqslant j<s$, then

$$
b_{i j}=\left\{\begin{array}{l}
w_{4(j+m)+3 \rightarrow 4(j+m+s+1)+2} \otimes e_{4 j}, \quad i=j \\
-w_{4(j+m+1) \rightarrow 4(j+m+s+1)+2} \otimes w_{4 j \rightarrow 4(j+s)+1}, \quad i=j+2 s \\
0, \quad \text { otherwise. }
\end{array}\right.
$$

If $s \leqslant j<2 s$, then

$$
b_{i j}=\left\{\begin{array}{l}
w_{4(j+m+1) \rightarrow 4(j+m+s+1)+2} \otimes w_{4 j \rightarrow 4\left(j+s-s f_{0}(j, s)\right)+1}, \quad i=j \\
0, \quad \text { otherwise. }
\end{array}\right.
$$

If $2 s \leqslant j<4 s$, then

$$
b_{i j}=\left\{\begin{array}{l}
\kappa_{1} w_{4(j+m+1) \rightarrow 4(j+m+1)+3} \otimes e_{4 j+1}, \quad i=j-s \\
-\kappa_{1} e_{4(j+m+1)+3} \otimes w_{4 j+1 \rightarrow 4(j+1)}, \quad i=(j+1)_{s}, j<3 s-1 \text { or } j=4 s-1 \\
0, \quad \text { otherwise },
\end{array}\right.
$$


where $\kappa_{1}=\kappa^{\ell+1}\left(\alpha_{3(j+m-3)}\right)$.

If $4 s \leqslant j<6 s$, then

$$
b_{i j}=\left\{\begin{array}{l}
\kappa_{1} w_{4(j+m+s+1)+1 \rightarrow 4(j+m+2)} \otimes e_{4 j+2}, \quad i=j-s ; \\
-\kappa_{1} w_{4(j+m+1)+3 \rightarrow 4(j+m+2)} \otimes w_{4 j+2 \rightarrow 4(j+1)}, \quad i=(j+1)_{s}, 5 s-1 \leqslant j<6 s-1 ; \\
0, \quad \text { otherwise, }
\end{array}\right.
$$

where $\kappa_{1}=f_{1}(j, 5 s) \kappa^{\ell}\left(\alpha_{3(j+m-1)}\right) \kappa^{\ell}\left(\gamma_{j+m-2}\right)$.

If $6 s \leqslant j<8 s$, then

$$
b_{i j}=\left\{\begin{array}{l}
w_{4(j+m+1)+3 \rightarrow 4(j+m+s+2)+1} \otimes w_{4 j+3 \rightarrow 4(j+1)}, \quad i=(j+1)_{s}, j<7 s-1 \text { or } j=8 s-1 ; \\
-w_{4(j+m+2) \rightarrow 4(j+m+s+2)+1} \otimes w_{4 j+3 \rightarrow 4(j+1)+1}, \\
\quad i=s+(j+1)_{s}, j<7 s-1 \text { or } j=8 s-1 ; \\
-w_{4(j+m+2) \rightarrow 4(j+m+s+2)+1} \otimes w_{4 j+3 \rightarrow 4(j+s+1)+1}, \\
\quad i=s+(j+1)_{s}, 7 s-1 \leqslant j<8 s-1 ; \\
0, \quad \text { otherwise. }
\end{array}\right.
$$

(11) If $r_{0}=10$, then $\Omega^{10}\left(Y_{t}^{(11)}\right)$ is described with $(9 s \times 6 s)$-matrix with the following elements $b_{i j}$ :

If $0 \leqslant j<s$, then

$$
b_{i j}=\left\{\begin{array}{l}
-\kappa_{1} w_{4(j+m) \rightarrow 4(j+m)+3} \otimes e_{4 j}, \quad i=j ; \\
\kappa_{1} w_{4(j+m+s)+1 \rightarrow 4(j+m)+3} \otimes w_{4 j \rightarrow 4 j+1}, \quad i=j+s \\
-\kappa_{1} w_{4(j+m)+1 \rightarrow 4(j+m)+3} \otimes w_{4 j \rightarrow 4(j+s)+1}, \quad i=j+2 s \\
-\kappa_{1} w_{4(j+m+s)+2 \rightarrow 4(j+m)+3} \otimes w_{4 j \rightarrow 4 j+2}, \quad i=j+3 s \\
\kappa_{1} w_{4(j+m)+2 \rightarrow 4(j+m)+3} \otimes w_{4 j \rightarrow 4(j+s)+2}, \quad i=j+4 s \\
-\kappa_{1} e_{4(j+m)+3} \otimes w_{4 j \rightarrow 4 j+3}, \quad i=j+5 s \\
0, \quad \text { otherwise, }
\end{array}\right.
$$

where $\kappa_{1}=\kappa^{\ell+1}\left(\alpha_{3(j+m-4)}\right)$.

If $s \leqslant j<2 s$, then

$$
b_{i j}=\left\{\begin{array}{l}
\kappa_{1} w_{4(j+m) \rightarrow 4(j+m+1)} \otimes e_{4 j}, \quad i=j-s ; \\
-\kappa_{1} w_{4(j+m+s)+1 \rightarrow 4(j+m+1)} \otimes w_{4 j \rightarrow 4 j+1}, \quad i=j+s \\
\kappa_{1} w_{4(j+m)+2 \rightarrow 4(j+m+1)} \otimes w_{4 j \rightarrow 4(j+s)+2}, \quad i=j+2 s \\
-\kappa_{1} w_{4(j+m+s)+2 \rightarrow 4(j+m+1)} \otimes w_{4 j \rightarrow 4 j+2}, \quad i=j+3 s \\
\kappa_{1} w_{4(j+m)+3 \rightarrow 4(j+m+1)} \otimes w_{4 j \rightarrow 4 j+3}, \quad i=j+4 s \\
0, \quad \text { otherwise, }
\end{array}\right.
$$

where $\kappa_{1}=\kappa^{\ell+1}\left(\alpha_{3(j+m-3)}\right)$. 
If $2 s \leqslant j<3 s-1$, then

$$
b_{i j}=\left\{\begin{array}{l}
-w_{4(j+m+1) \rightarrow 4(j+m+1)+1} \otimes w_{4 j+1 \rightarrow 4(j+1)}, \quad i=(j+1)_{s} \\
0, \quad \text { otherwise. }
\end{array}\right.
$$

If $3 s-1 \leqslant j<4 s-1$, then $b_{i j}=0$.

If $4 s-1 \leqslant j<4 s$, then

$$
b_{i j}=\left\{\begin{array}{l}
-w_{4(j+m+1) \rightarrow 4(j+m+1)+1} \otimes w_{4 j+1 \rightarrow 4(j+1)}, \quad i=(j+1)_{s} \\
0, \quad \text { otherwise. }
\end{array}\right.
$$

If $4 s \leqslant j<5 s$, then

$$
b_{i j}=\left\{\begin{array}{l}
-w_{4(j+m+s)+2 \rightarrow 4(j+m+s+1)+1} \otimes e_{4 j+2}, \quad i=j-s \\
-w_{4(j+m)+3 \rightarrow 4(j+m+s+1)+1} \otimes w_{4 j+2 \rightarrow 4 j+3}, \quad i=j+s ; \\
-w_{4(j+m+1) \rightarrow 4(j+m+s+1)+1} \otimes w_{4 j+2 \rightarrow 4(j+1)}, \quad i=(j+1)_{s}, j<5 s-1 ; \\
0, \quad \text { otherwise. }
\end{array}\right.
$$

If $5 s \leqslant j<6 s-1$, then $b_{i j}=0$.

If $6 s-1 \leqslant j<6 s$, then

$$
b_{i j}=\left\{\begin{array}{l}
w_{4(j+m+1) \rightarrow 4(j+m+s+1)+2} \otimes w_{4(j+s)+2 \rightarrow 4(j+1)}, \quad i=(j+1)_{s} ; \\
0, \quad \text { otherwise. }
\end{array}\right.
$$

If $6 s \leqslant j<7 s$, then

$$
b_{i j}=\left\{\begin{array}{l}
-w_{4(j+m)+2 \rightarrow 4(j+m+1)+1} \otimes e_{4(j+s)+2}, \quad i=j-2 s \\
w_{4(j+m)+3 \rightarrow 4(j+m+1)+1} \otimes w_{4(j+s)+2 \rightarrow 4 j+3}, \quad i=j-s ; \\
-w_{4(j+m+1) \rightarrow 4(j+m+1)+1} \otimes w_{4(j+s)+2 \rightarrow 4(j+1)}, \quad i=(j+1)_{s}, j=7 s-1 ; \\
0, \quad \text { otherwise. }
\end{array}\right.
$$

If $7 s \leqslant j<8 s-1$, then

$$
b_{i j}=\left\{\begin{array}{l}
w_{4(j+m+1) \rightarrow 4(j+m+1)+2} \otimes w_{4 j+2 \rightarrow 4(j+1)}, \quad i=(j+1)_{s} ; \\
0, \quad \text { otherwise. }
\end{array}\right.
$$

If $8 s-1 \leqslant j<8 s$, then $b_{i j}=0$.

If $8 s \leqslant j<9 s$, then

$$
b_{i j}= \begin{cases}\kappa_{1} w_{4(j+m+s+1+s f(j, 9 s-1))+1 \rightarrow 4(j+m+1)+3} \otimes w_{4 j+3 \rightarrow 4(j+1+s f(j, 9 s-1))+1}, & i=s+(j+1)_{s} \\ -\kappa_{1} w_{4(j+m+1+s f(j, 9 s-1))+1 \rightarrow 4(j+m+1)+3} \otimes w_{4 j+3 \rightarrow 4(j+s+1+s f(j, 9 s-1))+1}, & i=2 s+(j+1)_{s} \\ -\kappa_{1} w_{4(j+m+s+1+s f(j, 9 s-1))+2 \rightarrow 4(j+m+1)+3} \otimes w_{4 j+3 \rightarrow 4(j+1+s f(j, 9 s-1))+2}, & i=3 s+(j+1)_{s} \\ \kappa_{1} w_{4(j+m+1+s f(j, 9 s-1))+2 \rightarrow 4(j+m+1)+3} \otimes w_{4 j+3 \rightarrow 4(j+s+1+s f(j, 9 s-1))+2}, & i=4 s+(j+1)_{s} \\ -\kappa_{1} e_{4(j+m+1)+3} \otimes w_{4 j+3 \rightarrow 4(j+1)+3}, \quad i=5 s+(j+1)_{s} & \\ 0, \quad \text { otherwise, } & \end{cases}
$$


where $\kappa_{1}=-\kappa^{\ell+1}\left(\alpha_{3(j+m-3)}\right)$.

(II) Represent an arbitrary $t_{0} \in \mathbb{N}$ in the form $t_{0}=11 \ell_{0}+r_{0}$, where $0 \leqslant r_{0} \leqslant 10$. Then $\Omega^{t_{0}}\left(Y_{t}^{(11)}\right)$ is a $\Omega^{r_{0}}\left(Y_{t}^{(11)}\right)$, whose left components twisted by $\sigma^{\ell_{0}}$, and coefficients multiplied by $(-1)^{\ell_{0}}$.

Proposition 28 (Translates for the case 12). (I) Let $r_{0} \in \mathbb{N}, r_{0}<11$. $r_{0}$-translates of the elements $Y_{t}^{(12)}$ are described by the following way.

(1) If $r_{0}=0$, then $\Omega^{0}\left(Y_{t}^{(12)}\right)$ is described with $(8 s \times 6 s)$-matrix with the following two nonzero elements:

$$
\begin{aligned}
& b_{3 s, 4 s}=\kappa^{\ell}\left(\alpha_{3(j+m+2)}\right) w_{4(j+m)+2 \rightarrow 4(j+m)+3} \otimes e_{4 j+2} ; \\
& b_{4 s, 5 s}=\kappa^{\ell}\left(\alpha_{3(j+m+2)}\right) w_{4(j+m)+2 \rightarrow 4(j+m)+3} \otimes e_{4 j+2} .
\end{aligned}
$$

(2) If $r_{0}=1$, then $\Omega^{1}\left(Y_{t}^{(12)}\right)$ is described with $(9 s \times 7 s)$-matrix with the following nonzero elements:

$$
\begin{gathered}
b_{j,(3)_{s}}=-\kappa_{1} w_{4(j+m)+1 \rightarrow 4(j+m+1)} \otimes e_{4 j} ; \\
b_{j+s,(3)_{s}}=\kappa_{1} w_{4(j+m+s)+1 \rightarrow 4(j+m+1)} \otimes e_{4 j} ; \\
b_{j+2 s,(3)_{s}}=-\kappa_{1} w_{4(j+m)+2 \rightarrow 4(j+m+1)} \otimes w_{4 j \rightarrow 4 j+1} ; \\
b_{j+3 s,(3)_{s}}=\kappa_{1} w_{4(j+m+s)+2 \rightarrow 4(j+m+1)} \otimes w_{4 j \rightarrow 4(j+s)+1} ; \\
b_{j, 2 s+(3)_{s}}=-w_{4(j+m)+2 \rightarrow 4(j+m+1)+2} \otimes e_{4 j+1} ; \\
b_{j-s, 4 s+(3)_{s}}=-w_{4(j+m+s)+2 \rightarrow 4(j+m+s+1)+2} \otimes e_{4(j+s)+1} ; \\
b_{(j+1)_{s}+f(s, 1), 7 s+(3)_{s}-(1)_{s}}=-\kappa_{2} w_{4(j+m+s+1)+1 \rightarrow 4(j+m+1)+3} \otimes w_{4 j+3 \rightarrow 4(j+1)} ; \\
b_{s+(j+1)_{s}-f(s, 1), 7 s+(3)_{s}-(1)_{s}}=\kappa_{2} w_{4(j+m+1)+1 \rightarrow 4(j+m+1)+3} \otimes w_{4 j+3 \rightarrow 4(j+1)} ; \\
b_{2 s+(j+1)_{s}+f(s, 1), 7 s+(3)_{s}-(1)_{s}}=-\kappa_{2} w_{4(j+m+s+1)+2 \rightarrow 4(j+m+1)+3} \otimes w_{4 j+3 \rightarrow 4(j+s+1)+1} ; \\
b_{3 s+(j+1)_{s}-f(s, 1), 7 s+(3)_{s}-(1)_{s}}=\kappa_{2} w_{4(j+m+1)+2 \rightarrow 4(j+m+1)+3} \otimes w_{4 j+3 \rightarrow 4(j+1)+1} ;
\end{gathered}
$$

where $\kappa_{1}=\kappa^{\ell}\left(\alpha_{3\left((3)_{s}+m+3\right)}\right), \kappa_{2}=-\kappa^{\ell}\left(\alpha_{3\left(7 s+(3)_{s}-(1)_{s}+m\right)}\right)$.

(3) If $r_{0}=2$, then $\Omega^{2}\left(Y_{t}^{(12)}\right)$ is described with $(8 s \times 6 s)$-matrix with the following two nonzero elements:

$$
\begin{gathered}
b_{(j+1)_{s}, 2 s+(2)_{s}}=-f_{2}(s, 1) \kappa^{\ell}\left(\alpha_{3(j+m+2)}\right) e_{4(j+m)+3} \otimes w_{4 j+1 \rightarrow 4(j+1)} \\
b_{(j+1)_{s}, 3 s+(2)_{s}}=f_{2}(s, 1) \kappa^{\ell}\left(\alpha_{3(j+m+2)}\right) e_{4(j+m)+3} \otimes w_{4 j+1 \rightarrow 4(j+1)} .
\end{gathered}
$$

(4) If $r_{0}=3$, then $\Omega^{3}\left(Y_{t}^{(12)}\right)$ is described with $(6 s \times 8 s)$-matrix with the following two nonzero elements:

$$
\begin{aligned}
b_{s+(j+1)_{s}-f(s, 1), s+(2)_{s}} & =-f_{2}(s, 1) e_{4(j+m+1)+2} \otimes w_{4(j+s)+1 \rightarrow 4(j+1)} ; \\
b_{(j+1)_{s}+f(s, 1), 2 s+(2)_{s}} & =f_{2}(s, 1) e_{4(j+m+1)+2} \otimes w_{4(j+s)+1 \rightarrow 4(j+1)} .
\end{aligned}
$$

(5) If $r_{0}=4$, then $\Omega^{4}\left(Y_{t}^{(12)}\right)$ is described with $(7 s \times 9 s)$-matrix with the following nonzero elements:

$$
\begin{gathered}
b_{(j+1)_{s},(2)_{s}}=-\kappa^{\ell}\left(\alpha_{3(j+m+2)}\right) e_{4(j+m)+3} \otimes w_{4 j \rightarrow 4(j+1)} ; \\
b_{(j+1)_{s}, s+(2)_{s}+f(s, 1)}=-f_{2}(s, 1) \kappa^{\ell}\left(\alpha_{3(j+m+2)}\right) w_{4(j+m)+3 \rightarrow 4(j+m+1)} \otimes w_{4(j+s)+1 \rightarrow 4(j+1)} \\
b_{s+(j+1)_{s}, s+(2)_{s}+f(s, 1)}=f_{2}(s, 1) \kappa^{\ell}\left(\alpha_{3(j+m+2)}\right) e_{4(j+m+1)} \otimes w_{4(j+s)+1 \rightarrow 4(j+1)} ; \\
b_{s+(j+1)_{s}, 2 s+(2)_{s}-f(s, 1)}=-f_{2}(s, 1) \kappa^{\ell}\left(\alpha_{3(j+m+2)}\right) e_{4(j+m+1)} \otimes w_{4(j+s)+1 \rightarrow 4(j+1)} .
\end{gathered}
$$

(6) If $r_{0}=5$, then $\Omega^{5}\left(Y_{t}^{(12)}\right)$ is described with $(6 s \times 8 s)$-matrix with the following two nonzero elements:

$$
b_{(j+1)_{s}+f(s, 1), s+(2)_{s}}=e_{4(j+m+s+1)+1} \otimes w_{4(j+s)+1 \rightarrow 4(j+1)}
$$


HOCHSCHILD COHOMOLOGY RING FOR SELF-INJECTIVE ALGEBRAS OF TREE CLASS $E_{6}$. II. 97

$$
b_{s+(j+1)_{s}-f(s, 1), 2 s+(2)_{s}}=e_{4(j+m+s+1)+1} \otimes w_{4(j+s)+1 \rightarrow 4(j+1)} .
$$

(7) If $r_{0}=6$, then $\Omega^{6}\left(Y_{t}^{(12)}\right)$ is described with $(6 s \times 9 s)$-matrix with the following nonzero elements:

$$
\begin{gathered}
b_{(j+1)_{s},(2)_{s}}=-\kappa^{\ell}\left(\alpha_{3(j+m+3)}\right) e_{4(j+m+1)} \otimes w_{4 j \rightarrow 4(j+1)} ; \\
b_{j, s+(2)_{s}}=w_{4(j+m+s)+1 \rightarrow 4(j+m+s+1)+1} \otimes e_{4(j+s)+1} ; \\
b_{j+s, 2 s+(2)_{s}}=w_{4(j+m+s)+1 \rightarrow 4(j+m+s+1)+1} \otimes e_{4(j+s)+1} .
\end{gathered}
$$

(8) If $r_{0}=7$, then $\Omega^{7}\left(Y_{t}^{(12)}\right)$ is described with $(7 s \times 8 s)$-matrix with the following nonzero elements:

$$
\begin{gathered}
b_{j+2 s,(2)_{s}}=-w_{4(j+m)+3 \rightarrow 4(j+m+1)+1} \otimes w_{4 j \rightarrow 4 j+1} ; \\
b_{j+4 s,(2)_{s}}=-w_{4(j+m+1) \rightarrow 4(j+m+1)+1} \otimes w_{4 j \rightarrow 4 j+2} ; \\
b_{j+5 s,(2)_{s}}=-w_{4(j+m+1) \rightarrow 4(j+m+1)+1} \otimes w_{4 j \rightarrow 4(j+s)+2} ; \\
b_{j, s+(2)_{s}}=w_{4(j+m)+2 \rightarrow 4(j+m+1)+1} \otimes e_{4 j} ; \\
b_{j+s, s+(2)_{s}}=-w_{4(j+m)+3 \rightarrow 4(j+m+1)+1} \otimes w_{4 j \rightarrow 4(j+s)+1} ; \\
b_{j+3 s, s+(2)_{s}}=-w_{4(j+m+1) \rightarrow 4(j+m+1)+1} \otimes w_{4 j \rightarrow 4(j+s)+2} ; \\
b_{j+4 s, s+(2)_{s}}=-w_{4(j+m+1) \rightarrow 4(j+m+1)+1} \otimes w_{4 j \rightarrow 4 j+2} .
\end{gathered}
$$

(9) If $r_{0}=8$ and $s=2$, then $\Omega^{8}\left(Y_{t}^{(12)}\right)$ is described with $(6 s \times 6 s)$-matrix with the following nonzero elements:

$$
\begin{gathered}
b_{j, s+1}=-w_{4(j+m+s)+2 \rightarrow 4(j+m+s+1)+2} \otimes e_{4(j+2)+1} ; \\
b_{s+(j+1)_{s}, 2 s+1}=-e_{4(j+m+s+1)+2} \otimes w_{4(j+2)+1 \rightarrow 4(j+s+1)+1} ; \\
b_{4 s+(j+1)_{s}, 3 s+1}=-e_{4(j+m+1)+1} \otimes w_{4(j+s)+2 \rightarrow 4(j+s+1)+2} ; \\
b_{(j+1)_{s}, 4 s+1}=-w_{4(j+m)+3 \rightarrow 4(j+m+1)+1} \otimes w_{4(j+s)+2 \rightarrow 4(j+1)} ; \\
b_{3 s+(j+1)_{s}, 4 s+1}=-e_{4(j+m+1)+1} \otimes w_{4(j+s)+2 \rightarrow 4(j+s+1)+2} ; \\
b_{2 s+(j+1)_{s}, 5 s+1}=-w_{4(j+m+s+1)+2 \rightarrow 4(j+m)} \otimes w_{4 j+3 \rightarrow 4(j+s+1)+1} ; \\
b_{3 s+(j+1)_{s}, 5 s+1}=-w_{4(j+m+s+1)+1 \rightarrow 4(j+m)} \otimes w_{4 j+3 \rightarrow 4(j+1)+2} .
\end{gathered}
$$

(10) If $r_{0}=8$ and $s \neq 2$, then $\Omega^{8}\left(Y_{t}^{(12)}\right)$ is described with $(6 s \times 6 s)$-matrix with the following nonzero elements:

$$
\begin{aligned}
& b_{s+(j+1)_{s}, s+1}=-e_{4(j+m+s+1)+2} \otimes w_{4(j+s)+1 \rightarrow 4(j+s+1)+1} ; \\
& b_{j, 2 s-f_{2}(s, 1)}=-w_{4(j+m+s)+2 \rightarrow 4(j+m+s+1)+2} \otimes e_{4(j+s)+1} ; \\
& b_{(j+1)_{s}, 3 s+1}=-w_{4(j+m)+3 \rightarrow 4(j+m+1)+1} \otimes w_{4(j+s)+2 \rightarrow 4(j+1)} ; \\
& b_{3 s+(j+1)_{s}, 3 s+1}=-e_{4(j+m+1)+1} \otimes w_{4(j+s)+2 \rightarrow 4(j+s+1)+2} ; \\
& b_{4 s+(j+1)_{s}, 4 s-f_{2}(s, 1)}=-e_{4(j+m+1)+1} \otimes w_{4(j+s)+2 \rightarrow 4(j+s+1)+2} ; \\
& b_{2 s+(j+1)_{s}, 5 s+(1)_{s}}=-f_{2}(s, 1) \kappa^{\ell+1}\left(\alpha_{3(j+m-1)}\right) w_{4(j+m+1+s f(s, 1))+2 \rightarrow 4(j+m+2)} \otimes w_{4 j+3 \rightarrow 4(j+1+s f(s, 1))+1} \text {; } \\
& b_{3 s+(j+1)_{s}, 5 s+(1)_{s}}=-f_{2}(s, 1) \kappa^{\ell+1}\left(\alpha_{3(j+m-1)}\right) w_{4(j+m+1+s f(s, 1))+1 \rightarrow 4(j+m+2)} \otimes w_{4 j+3 \rightarrow 4(j+s+1+s f(s, 1))+2} \text {. }
\end{aligned}
$$

(11) If $r_{0}=9$ and $s=1$, then $\Omega^{9}\left(Y_{t}^{(12)}\right)$ is described with $(8 s \times 7 s)$-matrix with the following nonzero elements:

$$
\begin{gathered}
b_{j+s, 0}=w_{4(j+m) \rightarrow 4(j+m+1)+2} \otimes w_{4 j \rightarrow 4 j+1} ; \\
b_{j+2 s, 0}=-w_{4(j+m) \rightarrow 4(j+m+1)+2} \otimes w_{4 j \rightarrow 4(j+1)+1} ; \\
b_{j-3 s, 3 s}=\kappa^{\ell+1}\left(\alpha_{3(j+m)}\right) e_{4(j+m)+3} \otimes w_{4 j+1 \rightarrow 4 j} ; \\
b_{j-4 s, 4 s}=\kappa^{\ell+1}\left(\alpha_{3(j+m)}\right) w_{4(j+m)+3 \rightarrow 4(j+m)} \otimes w_{4 j+2 \rightarrow 4 j} ;
\end{gathered}
$$




$$
b_{j-6 s, 6 s}=-w_{4(j+m)+3 \rightarrow 4(j+m)+1} \otimes w_{4 j+3 \rightarrow 4 j} .
$$

(12) If $r_{0}=9$ and $s=2$, then $\Omega^{9}\left(Y_{t}^{(12)}\right)$ is described with $(8 s \times 7 s)$-matrix with the following nonzero elements:

$$
\begin{gathered}
b_{j+s, 1}=w_{4(j+m+1) \rightarrow 4(j+m+1)+2} \otimes w_{4 j \rightarrow 4 j+1} ; \\
b_{j+2 s, 1}=-w_{4(j+m+1) \rightarrow 4(j+m+1)+2} \otimes w_{4 j \rightarrow 4(j+2)+1} ; \\
b_{(j+1)_{s}, 3 s+1}=e_{4(j+m+1)+3} \otimes w_{4 j+1 \rightarrow 4(j+1)} ; \\
b_{(j+1)_{s}, 4 s+1}=w_{4(j+m+1)+3 \rightarrow 4(j+m)} \otimes w_{4 j+2 \rightarrow 4(j+1)} ; \\
b_{(j+1)_{s}, 6 s+1}=-w_{4(j+m+1)+3 \rightarrow 4(j+m+2)+1} \otimes w_{4 j+3 \rightarrow 4(j+1)} .
\end{gathered}
$$

(13) If $r_{0}=9$ and $s>2$, then $\Omega^{9}\left(Y_{t}^{(12)}\right)$ is described with $(8 s \times 7 s)$-matrix with the following nonzero elements:

$$
\begin{gathered}
b_{j+f(s, 1), s+1}=w_{4(j+m+1) \rightarrow 4(j+m+1)+2} \otimes w_{4 j \rightarrow 4(j+s)+1} \\
b_{j+s+f(s, 1), s+1}=-w_{4(j+m+1) \rightarrow 4(j+m+1)+2} \otimes w_{4 j \rightarrow 4 j+1} \\
b_{j-s, 2 s+1}=-\kappa^{\ell+1}\left(\alpha_{3(j+m)}\right) w_{4(j+m+1) \rightarrow 4(j+m+1)+3} \otimes e_{4 j+1} \\
b_{j+3 s-f(s, 1), 2 s+1}=\kappa^{\ell+1}\left(\alpha_{3(j+m)}\right) w_{4(j+m+1)+2 \rightarrow 4(j+m+1)+3} \otimes w_{4 j+1 \rightarrow 4 j+3} \\
b_{(j+1)_{s}, 5 s+1}=-\kappa^{\ell+1}\left(\alpha_{3(j+m-1)}\right) w_{4(j+m+1)+3 \rightarrow 4(j+m+2)} \otimes w_{4 j+2 \rightarrow 4(j+1)} \\
b_{(j+1)_{s}, 7 s+1}=-w_{4(j+m+1)+3 \rightarrow 4(j+m+2)+1} \otimes w_{4 j+3 \rightarrow 4(j+1)}
\end{gathered}
$$

(14) If $r_{0}=10$ and $s=1$, then $\Omega^{10}\left(Y_{t}^{(12)}\right)$ is described with $(9 s \times 6 s)$-matrix with the following nonzero elements:

$$
\begin{gathered}
b_{j+s, 0}=-\kappa_{1} w_{4(j+m+1)+1 \rightarrow 4(j+m)+3} \otimes w_{4 j \rightarrow 4 j+1} ; \\
b_{j+2 s, 0}=\kappa_{1} w_{4(j+m)+1 \rightarrow 4(j+m)+3} \otimes w_{4 j \rightarrow 4(j+1)+1} ; \\
b_{j+3 s, 0}=\kappa_{1} w_{4(j+m+1)+2 \rightarrow 4(j+m)+3} \otimes w_{4 j \rightarrow 4 j+2} ; \\
b_{j+4 s, 0}=-\kappa_{1} w_{4(j+m)+2 \rightarrow 4(j+m)+3} \otimes w_{4 j \rightarrow 4(j+1)+2} ; \\
b_{j+5 s, 0}=\kappa_{1} e_{4(j+m)+3} \otimes w_{4 j \rightarrow 4 j+3} ; \\
b_{j, s}=-\kappa_{2} w_{4(j+m)+1 \rightarrow 4(j+m)} \otimes w_{4 j \rightarrow 4(j+1)+1} ; \\
b_{j+s, s}=\kappa_{2} w_{4(j+m+1)+1 \rightarrow 4(j+m)} \otimes w_{4 j \rightarrow 4 j+1} ; \\
b_{j+2 s, s}=\kappa_{2} w_{4(j+m)+2 \rightarrow 4(j+m)} \otimes w_{4 j \rightarrow 4(j+1)+2} ; \\
b_{j+3 s, s}=-\kappa_{2} w_{4(j+m+1)+2 \rightarrow 4(j+m)} \otimes w_{4 j \rightarrow 4 j+2} ; \\
b_{j+4 s, s}=\kappa_{2} w_{4(j+m)+3 \rightarrow 4(j+m)} \otimes w_{4 j \rightarrow 4 j+3} ; \\
b_{j-s, 2 s}=w_{4(j+m+1)+1 \rightarrow 4(j+m)+1} \otimes e_{4 j+1} ; \\
b_{j+s, 2 s}=-w_{4(j+m+1)+2 \rightarrow 4(j+m)+1} \otimes w_{4 j+1 \rightarrow 4 j+2} ; \\
b_{j+3 s, 2 s}=-w_{4(j+m)+3 \rightarrow 4(j+m)+1} \otimes w_{4 j+1 \rightarrow 4 j+3} ; \\
b_{j-3 s, 5 s}=-w_{4(j+m+1)+1 \rightarrow 4(j+m+1)+2} \otimes w_{4(j+1)+2 \rightarrow 4 j+1} ; \\
b_{j-2 s, 5 s}=-w_{4(j+m)+2 \rightarrow 4(j+m+1)+2} \otimes e_{4(j+1)+2} ; \\
b_{j-s, 5 s}=e_{4(j+m+1)+2} \otimes w_{4(j+1)+2 \rightarrow 4 j+2} ; \\
b_{j, 5 s}=-w_{4(j+m)+3 \rightarrow 4(j+m+1)+2} \otimes w_{4(j+1)+2 \rightarrow 4 j+3} \\
b_{j-3 s, 7 s}=-w_{4(j+m+1)+2 \rightarrow 4(j+m)+2} \otimes e_{4 j+2} \\
b_{j-2 s, 7 s}=w_{4(j+m)+3 \rightarrow 4(j+m)+2} \otimes w_{4 j+2 \rightarrow 4 j+3} \\
b_{j-8 s, 8 s}=-\kappa^{\ell+1}\left(\alpha_{3(j+m)}\right) w_{4(j+m) \rightarrow 4(j+m)+3} \otimes w_{4 j+3 \rightarrow 4 j}
\end{gathered}
$$

where $\kappa_{1}=-\kappa^{\ell+1}\left(\alpha_{3 m}\right), \kappa_{2}=-\kappa^{\ell+1}\left(\alpha_{3(s+m)}\right)$. 
(15) If $r_{0}=10$ and $s=2$, then $\Omega^{10}\left(Y_{t}^{(12)}\right)$ is described with $(9 s \times 6 s)$-matrix with the following nonzero elements:

$$
\begin{gathered}
b_{j+s, 1}=w_{4(j+m+2)+1 \rightarrow 4(j+m)+3} \otimes w_{4 j \rightarrow 4 j+1} ; \\
b_{j+2 s, 1}=-w_{4(j+m)+1 \rightarrow 4(j+m)+3} \otimes w_{4 j \rightarrow 4(j+2)+1} ; \\
b_{j+3 s, 1}=-w_{4(j+m+s)+2 \rightarrow 4(j+m)+3} \otimes w_{4 j \rightarrow 4 j+2} ; \\
b_{j+4 s, 1}=w_{4(j+m)+2 \rightarrow 4(j+m)+3} \otimes w_{4 j \rightarrow 4(j+s)+2} ; \\
b_{j+5 s, 1}=-e_{4(j+m)+3} \otimes w_{4 j \rightarrow 4 j+3} ; \\
b_{j, s+1}=w_{4(j+m)+1 \rightarrow 4(j+m+1)} \otimes w_{4 j \rightarrow 4(j+2)+1} ; \\
b_{j+s, s+1}=-w_{4(j+m+2)+1 \rightarrow 4(j+m+1)} \otimes w_{4 j \rightarrow 4 j+1} ; \\
b_{j+2 s, s+1}=-w_{4(j+m)+2 \rightarrow 4(j+m+1)} \otimes w_{4 j \rightarrow 4(j+s)+2} ; \\
b_{j+3 s, s+1}=w_{4(j+m+s)+2 \rightarrow 4(j+m+1)} \otimes w_{4 j \rightarrow 4 j+2} ; \\
b_{j+4 s, s+1}=-w_{4(j+m)+3 \rightarrow 4(j+m+1)} \otimes w_{4 j \rightarrow 4 j+3} ; \\
b_{j-s, 2 s+1}=w_{4(j+m+2)+1 \rightarrow 4(j+m+s+1)+1} \otimes e_{4 j+1} ; \\
b_{j+s, 2 s+1}=-w_{4(j+m+s)+2 \rightarrow 4(j+m+s+1)+1} \otimes w_{4 j+1 \rightarrow 4 j+2} ; \\
b_{j+3 s, 2 s+1}=-w_{4(j+m)+3 \rightarrow 4(j+m+s+1)+1} \otimes w_{4 j+1 \rightarrow 4 j+3} ; \\
b_{j-2 s, 5 s+1}=-w_{4(j+m)+2 \rightarrow 4(j+m+1)+2} \otimes e_{4(j+s)+2} ; \\
b_{j, 5 s+1}=-w_{4(j+m)+3 \rightarrow 4(j+m+1)+2} \otimes w_{4(j+s)+2 \rightarrow 4 j+3} ; \\
b_{2 s+(j+1)_{s}, 7 s}=-w_{4(j+m+s+1)+1 \rightarrow 4(j+m+s+1)+2} \otimes w_{4 j+2 \rightarrow 4(j+1)+1} ; \\
b_{4 s+(j+1)_{s}, 7 s}=e_{4(j+m+s+1)+2} \otimes w_{4 j+2 \rightarrow 4(j+1)+2} ; \\
b_{j-3 s, 8 s-1}=-w_{4(j+m+s)+2 \rightarrow 4(j+m+s+1)+2} \otimes e_{4 j+2} ; \\
b_{j-2 s, 8 s-1}=w_{4(j+m)+3 \rightarrow 4(j+m+s+1)+2} \otimes w_{4 j+2 \rightarrow 4 j+3} ; \\
b_{(j+1)_{s}, 8 s}=w_{4(j+m+1) \rightarrow 4(j+m+1)+3} \otimes w_{4 j+3 \rightarrow 4(j+1)} ; \\
b_{j-3 s, 8 s}=-w_{4(j+m)+3 \rightarrow 4(j+m+1)+3} \otimes e_{4 j+3} ; \\
b_{j-3 s, 9 s-1}=w_{4(j+m)+3 \rightarrow 4(j+m+1)+3} \otimes e_{4 j+3} .
\end{gathered}
$$

(16) If $r_{0}=10$ and $s>2$, then $\Omega^{10}\left(Y_{t}^{(12)}\right)$ is described with $(9 s \times 6 s)$-matrix with the following nonzero elements:

$$
\begin{gathered}
b_{j+2 s, 1}=-\kappa_{1} w_{4(j+m)+1 \rightarrow 4(j+m)+3} \otimes w_{4 j \rightarrow 4(j+s)+1} ; \\
b_{j+4 s, 1}=\kappa_{1} w_{4(j+m)+2 \rightarrow 4(j+m)+3} \otimes w_{4 j \rightarrow 4(j+s)+2} ; \\
b_{j, s+1}=-\kappa_{2} w_{4(j+m)+1 \rightarrow 4(j+m+1)} \otimes w_{4 j \rightarrow 4(j+s)+1} ; \\
b_{j+2 s, s+1}=\kappa_{2} w_{4(j+m)+2 \rightarrow 4(j+m+1)} \otimes w_{4 j \rightarrow 4(j+s)+2} ; \\
b_{j+4 s, s+1}=\kappa_{2} w_{4(j+m)+3 \rightarrow 4(j+m+1)} \otimes w_{4 j \rightarrow 4 j+3} ; \\
b_{j-s, 2 s+1}=w_{4(j+m+s)+1 \rightarrow 4(j+m+s+1)+1} \otimes e_{4 j+1} ; \\
b_{j+s, 2 s+1}=-w_{4(j+m+s)+2 \rightarrow 4(j+m+s+1)+1} \otimes w_{4 j+1 \rightarrow 4 j+2} ; \\
b_{j+3 s, 2 s+1}=-w_{4(j+m)+3 \rightarrow 4(j+m+s+1)+1} \otimes w_{4 j+1 \rightarrow 4 j+3} ; \\
b_{j-s, 3 s+1}=w_{4(j+m+s)+1 \rightarrow 4(j+m+s+1)+1} \otimes e_{4 j+1} ; \\
b_{j+s, 3 s+1}=-w_{4(j+m+s)+2 \rightarrow 4(j+m+s+1)+1} \otimes w_{4 j+1 \rightarrow 4 j+2} ; \\
b_{j+2 s, 3 s+1}=w_{4(j+m)+3 \rightarrow 4(j+m+s+1)+1} \otimes w_{4 j+1 \rightarrow 4 j+3} ; \\
b_{s+(j+1)_{s}, 5 s}=-w_{4(j+m+1)+1 \rightarrow 4(j+m+1)+2} \otimes w_{4(j+s)+2 \rightarrow 4(j+s+1)+1} ; \\
b_{3 s+(j+1)_{s}, 5 s}=e_{4(j+m+1)+2} \otimes w_{4(j+s)+2 \rightarrow 4(j+s+1)+2} ; \\
b_{j-2 s, 5 s+1}=-w_{4(j+m)+2 \rightarrow 4(j+m+1)+2} \otimes e_{4(j+s)+2} ;
\end{gathered}
$$


100

$$
\begin{gathered}
b_{j, 5 s+1}=-w_{4(j+m)+3 \rightarrow 4(j+m+1)+2} \otimes w_{4(j+s)+2 \rightarrow 4 j+3} ; \\
b_{j-3 s, 7 s+1}=-w_{4(j+m+s)+2 \rightarrow 4(j+m+s+1)+2} \otimes e_{4 j+2} ; \\
b_{j-2 s, 7 s+1}=w_{4(j+m)+3 \rightarrow 4(j+m+s+1)+2} \otimes w_{4 j+2 \rightarrow 4 j+3} ; \\
b_{(j+1)_{s}, 8 s}=-\kappa_{3} w_{4(j+m+1) \rightarrow 4(j+m+1)+3} \otimes w_{4 j+3 \rightarrow 4(j+1)} ; \\
b_{2 s+(j+1)_{s}, 8 s}=-\kappa_{3} w_{4(j+m+1)+1 \rightarrow 4(j+m+1)+3} \otimes w_{4 j+3 \rightarrow 4(j+s+1)+1} \\
b_{4 s+(j+1)_{s}, 8 s}=\kappa_{3} w_{4(j+m+1)+2 \rightarrow 4(j+m+1)+3} \otimes w_{4 j+3 \rightarrow 4(j+s+1)+2} ; \\
b_{j-3 s, 8 s}=\kappa_{3} w_{4(j+m)+3 \rightarrow 4(j+m+1)+3} \otimes e_{4 j+3} ; \\
b_{j-3 s, 8 s+1}=-\kappa^{\ell+1}\left(\alpha_{3(j+m-2)}\right) w_{4(j+m)+3 \rightarrow 4(j+m+1)+3} \otimes e_{4 j+3}
\end{gathered}
$$

where $\kappa_{1}=\kappa^{\ell+1}\left(\alpha_{3 m}\right), \kappa_{2}=\kappa^{\ell+1}\left(\alpha_{3(s+m-1)}\right), \kappa_{3}=\kappa^{\ell+1}\left(\alpha_{3 m}\right)$.

(II) Represent an arbitrary $t_{0} \in \mathbb{N}$ in the form $t_{0}=11 \ell_{0}+r_{0}$, where $0 \leqslant r_{0} \leqslant 10$. Then $\Omega^{t_{0}}\left(Y_{t}^{(12)}\right)$ is a $\Omega^{r_{0}}\left(Y_{t}^{(12)}\right)$, whose left components twisted by $\sigma^{\ell_{0}}$.

Proposition 29 (Translates for the case 13). (I) Let $r_{0} \in \mathbb{N}, r_{0}<11$. $r_{0}$-translates of the elements $Y_{t}^{(13)}$ are described by the following way.

(1) If $r_{0}=0$, then $\Omega^{0}\left(Y_{t}^{(13)}\right)$ is described with $(9 s \times 6 s)$-matrix with the following elements $b_{i j}$ : If $s \leqslant j<2 s$, then

$$
b_{i j}=\left\{\begin{array}{l}
e_{4(j+m+s)+1} \otimes e_{4(j+s)+1}, \quad i=j ; \\
0, \quad \text { otherwise. }
\end{array}\right.
$$

If $2 s \leqslant j<3 s$, then $b_{i j}=0$.

If $3 s \leqslant j<4 s$, then

$$
b_{i j}=\left\{\begin{array}{l}
e_{4(j+m)+1} \otimes e_{4 j+1}, \quad i=j-s \\
0, \quad \text { otherwise }
\end{array}\right.
$$

If $4 s \leqslant j<5 s$, then $b_{i j}=0$.

If $5 s \leqslant j<7 s$, then

$$
b_{i j}=\left\{\begin{array}{l}
e_{4(j+m+s)+2} \otimes e_{4(j+s)+2}, \quad i=j-2 s \\
0, \quad \text { otherwise. }
\end{array}\right.
$$

If $7 s \leqslant j<8 s$, then $b_{i j}=0$.

If $8 s \leqslant j<9 s$, then

$$
b_{i j}=\left\{\begin{array}{l}
w_{4(j+m)+3 \rightarrow 4(j+m+1)} \otimes e_{4 j+3}, \quad i=j-3 s \\
0, \quad \text { otherwise. }
\end{array}\right.
$$

(2) If $r_{0}=1$, then $\Omega^{1}\left(Y_{t}^{(13)}\right)$ is described with $(8 s \times 7 s)$-matrix with the following elements $b_{i j}$ : If $0 \leqslant j<2 s$, then

$$
b_{i j}=\left\{\begin{array}{l}
e_{4(j+m)+2} \otimes w_{4 j \rightarrow 4 j+1}, \quad i=j+2 s \\
0, \quad \text { otherwise. }
\end{array}\right.
$$


HOCHSCHILD COHOMOLOGY RING FOR SELF-INJECTIVE ALGEBRAS OF TREE CLASS $E_{6}$. II. 101 If $2 s \leqslant j<4 s$, then

$$
b_{i j}=\left\{\begin{array}{l}
w_{4(j+m)+2 \rightarrow 4(j+m)+3} \otimes e_{4 j+1}, \quad i=j \\
0, \quad \text { otherwise. }
\end{array}\right.
$$

If $4 s \leqslant j<6 s$, then

$$
b_{i j}=\left\{\begin{array}{l}
w_{4(j+m)+3 \rightarrow 4(j+m+1)} \otimes e_{4 j+2}, \quad i=j \\
0, \quad \text { otherwise }
\end{array}\right.
$$

If $6 s \leqslant j<8 s$, then

$$
b_{i j}=\left\{\begin{array}{l}
e_{4(j+m+1)+1} \otimes w_{4 j+3 \rightarrow 4(j+1)}, \quad i=(j+1)_{2 s} \\
w_{4(j+m+1) \rightarrow 4(j+m+1)+1} \otimes e_{4 j+3}, \quad i=6 s+(j)_{s} \\
0, \quad \text { otherwise. }
\end{array}\right.
$$

(3) If $r_{0}=2$, then $\Omega^{2}\left(Y_{t}^{(13)}\right)$ is described with $(6 s \times 6 s)$-matrix with the following elements $b_{i j}$ : If $s \leqslant j<3 s$, then

$$
b_{i j}=\left\{\begin{array}{l}
e_{4(j+m+s)+2} \otimes e_{4(j+s)+1}, \quad i=j \\
0, \quad \text { otherwise. }
\end{array}\right.
$$

If $3 s \leqslant j<5 s$, then

$$
b_{i j}=\left\{\begin{array}{l}
e_{4(j+m)+1} \otimes e_{4(j+s)+2}, \quad i=j \\
0, \quad \text { otherwise }
\end{array}\right.
$$

If $5 s \leqslant j<6 s$, then

$$
b_{i j}=\left\{\begin{array}{l}
w_{4(j+m)+3 \rightarrow 4(j+m+1)} \otimes w_{4 j+3 \rightarrow 4(j+1)}, \quad i=(j+1)_{s} \\
0, \quad \text { otherwise. }
\end{array}\right.
$$

(4) If $r_{0}=3$, then $\Omega^{3}\left(Y_{t}^{(13)}\right)$ is described with $(7 s \times 8 s)$-matrix with the following elements $b_{i j}$ : If $0 \leqslant j<s$, then

$$
b_{i j}=\left\{\begin{array}{l}
w_{4(j+m)+2 \rightarrow 4(j+m)+3} \otimes e_{4 j}, \quad i=j \\
w_{4(j+m+s)+2 \rightarrow 4(j+m)+3} \otimes e_{4 j}, \quad i=j+s \\
0, \quad \text { otherwise. }
\end{array}\right.
$$

If $s \leqslant j<3 s$, then

$$
b_{i j}=\left\{\begin{array}{l}
w_{4(j+m)+3 \rightarrow 4(j+m+1)} \otimes e_{4(j+s)+1}, \quad i=j+s \\
0, \quad \text { otherwise }
\end{array}\right.
$$


If $3 s \leqslant j<5 s$, then

$$
b_{i j}=\left\{\begin{array}{l}
w_{4(j+m+1) \rightarrow 4(j+m+1)+1} \otimes e_{4(j+s)+2}, \quad i=j+s ; \\
e_{4(j+m+1)+1} \otimes w_{4(j+s)+2 \rightarrow 4 j+3}, \quad i=6 s+(j)_{2 s} ; \\
0, \quad \text { otherwise. }
\end{array}\right.
$$

If $5 s \leqslant j<7 s$, then

$$
b_{i j}=\left\{\begin{array}{l}
e_{4(j+m+s+1)+2} \otimes w_{4 j+3 \rightarrow 4(j+1)}, \quad i=(j+s+1)_{2 s} ; \\
0, \quad \text { otherwise. }
\end{array}\right.
$$

(5) If $r_{0}=4$, then $\Omega^{4}\left(Y_{t}^{(13)}\right)$ is described with $(6 s \times 9 s)$-matrix with the following elements $b_{i j}$ : If $0 \leqslant j<s$, then

$$
b_{i j}=\left\{\begin{array}{l}
w_{4(j+m)-1 \rightarrow 4(j+m)} \otimes e_{4 j}, \quad i=j \\
0, \quad \text { otherwise }
\end{array}\right.
$$

If $s \leqslant j<3 s$, then

$$
b_{i j}=\left\{\begin{array}{l}
e_{4(j+m)+1} \otimes e_{4(j+s)+1}, \quad i=j+s \\
0, \quad \text { otherwise. }
\end{array}\right.
$$

If $3 s \leqslant j<5 s$, then

$$
b_{i j}=\left\{\begin{array}{l}
e_{4(j+m)+2} \otimes e_{4(j+s)+2}, \quad i=j+3 s-s f_{0}(j, 4 s) \\
0, \quad \text { otherwise. }
\end{array}\right.
$$

If $5 s \leqslant j<6 s$, then $b_{i j}=0$.

(6) If $r_{0}=5$, then $\Omega^{5}\left(Y_{t}^{(13)}\right)$ is described with $(6 s \times 8 s)$-matrix with the following elements $b_{i j}$ : If $0 \leqslant j<s$, then

$$
b_{i j}=\left\{\begin{array}{l}
e_{4(j+m+1)} \otimes w_{4 j \rightarrow 4 j+1}, \quad i=j+2 s \\
e_{4(j+m+1)} \otimes w_{4 j \rightarrow 4(j+s)+1}, \quad i=j+3 s \\
0, \quad \text { otherwise. }
\end{array}\right.
$$

If $s \leqslant j<3 s$, then

$$
b_{i j}=\left\{\begin{array}{l}
e_{4(j+m+1)+1} \otimes w_{4(j+s)+1 \rightarrow 4(j+1)}, \quad i=(j+1)_{2 s} ; \\
w_{4(j+m+1) \rightarrow 4(j+m+1)+1} \otimes e_{4(j+s)+1}, \quad i=j+s \\
0, \quad \text { otherwise. }
\end{array}\right.
$$

If $3 s \leqslant j<5 s$, then

$$
b_{i j}=\left\{\begin{array}{l}
w_{4(j+m)+3 \rightarrow 4(j+m+1)+2} \otimes e_{4(j+s)+2}, \quad i=j+s ; \\
e_{4(j+m+1)+2} \otimes w_{4(j+s)+2 \rightarrow 4 j+3}, \quad i=6 s+(j)_{2 s} ; \\
0, \quad \text { otherwise. }
\end{array}\right.
$$


HOCHSCHILD COHOMOLOGY RING FOR SELF-INJECTIVE ALGEBRAS OF TREE CLASS E. II. 103 If $5 s \leqslant j<6 s$, then

$$
b_{i j}=\left\{\begin{array}{l}
w_{4(j+m+s+1+s f(j, 6 s-1))+1 \rightarrow 4(j+m+1)+3} \otimes w_{4 j+3 \rightarrow 4(j+1)}, \quad i=(j+1)_{s} ; \\
w_{4(j+m+1+s f(j, 6 s-1))+1 \rightarrow 4(j+m+1)+3} \otimes w_{4 j+3 \rightarrow 4(j+1)}, \quad i=s+(j+1)_{s} \\
e_{4(j+m+1)+3} \otimes w_{4 j+3 \rightarrow 4(j+s+1+s f(j, 6 s-1))+2}, \quad i=4 s+(j+1)_{s} ; \\
e_{4(j+m+1)+3} \otimes w_{4 j+3 \rightarrow 4(j+1+s f(j, 6 s-1))+2}, \quad i=5 s+(j+1)_{s} ; \\
0, \quad \text { otherwise. }
\end{array}\right.
$$

(7) If $r_{0}=6$, then $\Omega^{6}\left(Y_{t}^{(13)}\right)$ is described with $(7 s \times 9 s)$-matrix with the following elements $b_{i j}$ : If $0 \leqslant j<2 s$, then

$$
b_{i j}=\left\{\begin{array}{l}
e_{4(j+m+s)+1} \otimes w_{4 j \rightarrow 4(j+s)+1}, \quad i=j+3 s f_{0}(j, s) \\
0, \quad \text { otherwise. }
\end{array}\right.
$$

If $2 s \leqslant j<4 s$, then

$$
b_{i j}=\left\{\begin{array}{l}
e_{4(j+m+s)+2} \otimes e_{4 j+1}, \quad i=j+s\left(1-f_{0}(j, 3 s)\right) \\
0, \quad \text { otherwise. }
\end{array}\right.
$$

If $4 s \leqslant j<6 s$, then

$$
b_{i j}=\left\{\begin{array}{l}
w_{4(j+m)+2 \rightarrow 4(j+m)+3} \otimes e_{4 j+2}, \quad i=j+s \\
e_{4(j+m)+3} \otimes w_{4 j+2 \rightarrow 4 j+3}, \quad i=7 s+(j)_{s} \\
0, \quad \text { otherwise. }
\end{array}\right.
$$

If $6 s \leqslant j<7 s$, then

$$
b_{i j}=\left\{\begin{array}{l}
e_{4(j+m+1)} \otimes w_{4 j+3 \rightarrow 4(j+1)}, \quad i=(j+1)_{s} \\
0, \quad \text { otherwise. }
\end{array}\right.
$$

(8) If $r_{0}=7$, then $\Omega^{7}\left(Y_{t}^{(13)}\right)$ is described with $(6 s \times 8 s)$-matrix with the following elements $b_{i j}$ : If $0 \leqslant j<s$, then

$$
b_{i j}=\left\{\begin{array}{l}
w_{4(j+m)+2 \rightarrow 4(j+m)+3} \otimes e_{4 j}, \quad i=j \\
w_{4(j+m+s)+2 \rightarrow 4(j+m)+3} \otimes e_{4 j}, \quad i=j+s \\
0, \quad \text { otherwise. }
\end{array}\right.
$$

If $s \leqslant j<3 s$, then

$$
b_{i j}=\left\{\begin{array}{l}
e_{4(j+m+1)+2} \otimes w_{4(j+s)+1 \rightarrow 4(j+1)}, \quad i=(j+1)_{2 s} \\
w_{4(j+m)+3 \rightarrow 4(j+m+1)+2} \otimes e_{4(j+s)+1}, \quad i=j+s \\
0, \quad \text { otherwise. }
\end{array}\right.
$$


If $3 s \leqslant j<5 s$, then

$$
b_{i j}=\left\{\begin{array}{l}
w_{4(j+m+1) \rightarrow 4(j+m+s+1)+1} \otimes e_{4(j+s)+2}, \quad i=j+s \\
e_{4(j+m+s+1)+1} \otimes w_{4(j+s)+2 \rightarrow 4 j+3}, \quad i=j+3 s \\
0, \quad \text { otherwise. }
\end{array}\right.
$$

If $5 s \leqslant j<6 s$, then

$$
b_{i j}=\left\{\begin{array}{l}
w_{4(j+m+1+s f(j, 6 s-1))+2 \rightarrow 4(j+m+2)} \otimes w_{4 j+3 \rightarrow 4(j+1)}, \quad i=s+(j+1)_{s} \\
e_{4(j+m+2)} \otimes w_{4 j+3 \rightarrow 4(j+s+1+s f(j, 6 s-1))+2}, \quad i=4 s+(j+1)_{s} \\
e_{4(j+m+2)} \otimes w_{4 j+3 \rightarrow 4(j+1+s f(j, 6 s-1))+2}, \quad i=5 s+(j+1)_{s} \\
0, \quad \text { otherwise. }
\end{array}\right.
$$

(9) If $r_{0}=8$, then $\Omega^{8}\left(Y_{t}^{(13)}\right)$ is described with $(8 s \times 6 s)$-matrix with the following elements $b_{i j}$ : If $0 \leqslant j<2 s$, then

$$
b_{i j}=\left\{\begin{array}{l}
w_{4(j+m)-1 \rightarrow 4(j+m+s)+2} \otimes e_{4 j}, \quad i=(j)_{s} \\
e_{4(j+m+s)+2} \otimes w_{4 j \rightarrow 4(j+s)+1}, \quad i=s+(j+s)_{2 s} \\
0, \quad \text { otherwise. }
\end{array}\right.
$$

If $2 s \leqslant j<4 s$, then

$$
b_{i j}=\left\{\begin{array}{l}
w_{4(j+m)+2 \rightarrow 4(j+m)+3} \otimes e_{4 j+1}, \quad i=j-s \\
0, \quad \text { otherwise. }
\end{array}\right.
$$

If $4 s \leqslant j<6 s$, then

$$
b_{i j}=\left\{\begin{array}{l}
w_{4(j+m)+3 \rightarrow 4(j+m+1)} \otimes w_{4 j+2 \rightarrow 4(j+1)}, \quad i=(j+1)_{s}, j<5 s-1 \text { or } j=6 s-1 \\
w_{4(j+m+s)+1 \rightarrow 4(j+m+1)} \otimes e_{4 j+2}, \quad i=j-s \\
e_{4(j+m+1)} \otimes w_{4 j+2 \rightarrow 4 j+3}, \quad i=5 s+(j)_{s} \\
0, \quad \text { otherwise. }
\end{array}\right.
$$

If $6 s \leqslant j<8 s$, then

$$
b_{i j}=\left\{\begin{array}{l}
e_{4(j+m+s+1)+1} \otimes w_{4 j+3 \rightarrow 4(j+1)+2}, \quad i=3 s+(j+1)_{2 s} \\
0, \quad \text { otherwise. }
\end{array}\right.
$$

(10) If $r_{0}=9$, then $\Omega^{9}\left(Y_{t}^{(13)}\right)$ is described with $(9 s \times 7 s)$-matrix with the following elements $b_{i j}$ :

If $s \leqslant j<2 s$, then

$$
b_{i j}=\left\{\begin{array}{l}
w_{4(j+m)+3 \rightarrow 4(j+m+1)} \otimes e_{4 j}, \quad i=j-s \\
0, \quad \text { otherwise }
\end{array}\right.
$$


HOCHSCHILD COHOMOLOGY RING FOR SELF-INJECTIVE ALGEBRAS OF TREE CLASS E $E_{6}$ II. 105 If $2 s \leqslant j<4 s$, then

$$
b_{i j}=\left\{\begin{array}{l}
w_{4(j+m+1) \rightarrow 4(j+m+1)+1} \otimes e_{4 j+1}, \quad i=j-s \\
0, \quad \text { otherwise. }
\end{array}\right.
$$

If $4 s \leqslant j<5 s$, then

$$
b_{i j}=\left\{\begin{array}{l}
e_{4(j+m+s+1)+1} \otimes e_{4 j+2}, \quad i=j-s \\
0, \quad \text { otherwise }
\end{array}\right.
$$

If $5 s \leqslant j<6 s$, then

$$
b_{i j}=\left\{\begin{array}{l}
e_{4(j+m+s+1)+2} \otimes w_{4(j+s)+2 \rightarrow 4 j+3}, \quad i=j \\
0, \quad \text { otherwise. }
\end{array}\right.
$$

If $6 s \leqslant j<7 s$, then

$$
b_{i j}=\left\{\begin{array}{l}
e_{4(j+m+1)+1} \otimes e_{4(j+s)+2}, \quad i=j-2 s \\
0, \quad \text { otherwise. }
\end{array}\right.
$$

If $7 s \leqslant j<8 s$, then

$$
b_{i j}=\left\{\begin{array}{l}
e_{4(j+m+1)+2} \otimes w_{4 j+2 \rightarrow 4 j+3}, \quad i=j-s \\
0, \quad \text { otherwise. }
\end{array}\right.
$$

If $8 s \leqslant j<9 s$, then

$$
b_{i j}=\left\{\begin{array}{l}
e_{4(j+m+1)+3} \otimes w_{4 j+3 \rightarrow 4(j+1)}, \quad i=(j+1)_{s} \\
0, \quad \text { otherwise. }
\end{array}\right.
$$

(11) If $r_{0}=10$, then $\Omega^{10}\left(Y_{t}^{(13)}\right)$ is described with $(8 s \times 6 s)$-matrix with the following elements $b_{i j}$ :

If $0 \leqslant j<2 s$, then

$$
b_{i j}=\left\{\begin{array}{l}
w_{4(j+m) \rightarrow 4(j+m+s)+1} \otimes e_{4 j}, \quad i=(j)_{s} \\
e_{4(j+m+s)+1} \otimes w_{4 j \rightarrow 4 j+1}, \quad i=j+s \\
0, \quad \text { otherwise. }
\end{array}\right.
$$

If $2 s \leqslant j<4 s$, then

$$
b_{i j}=\left\{\begin{array}{l}
w_{4(j+m+s)+1 \rightarrow 4(j+m+1)} \otimes e_{4 j+1}, \quad i=j-s \\
w_{4(j+m+s)+2 \rightarrow 4(j+m+1)} \otimes w_{4 j+1 \rightarrow 4 j+2}, \quad i=j+s \\
w_{4(j+m)+3 \rightarrow 4(j+m+1)} \otimes w_{4 j+1 \rightarrow 4 j+3}, \quad i=5 s+(j)_{s} \\
0, \quad \text { otherwise. }
\end{array}\right.
$$


If $4 s \leqslant j<6 s$, then

$$
b_{i j}=\left\{\begin{array}{l}
w_{4(j+m+s)+2 \rightarrow 4(j+m)+3} \otimes e_{4 j+2}, \quad i=j-s \\
e_{4(j+m)+3} \otimes w_{4 j+2 \rightarrow 4 j+3}, \quad i=5 s+(j)_{s} \\
0, \quad \text { otherwise. }
\end{array}\right.
$$

If $6 s \leqslant j<8 s$, then

$$
b_{i j}=\left\{\begin{array}{l}
w_{4(j+m+1) \rightarrow 4(j+m+s+1)+2} \otimes w_{4 j+3 \rightarrow 4(j+1)}, \quad i=(j+1)_{s} ; \\
w_{4(j+m+s+1)+1 \rightarrow 4(j+m+s+1)+2} \otimes w_{4 j+3 \rightarrow 4(j+1)+1}, \quad i=s+(j+1)_{2 s} \\
e_{4(j+m+s+1)+2} \otimes w_{4 j+3 \rightarrow 4(j+1)+2}, \quad i=3 s+(j+1)_{2 s}, \quad j<7 s \\
0, \quad \text { otherwise. }
\end{array}\right.
$$

If $8 s \leqslant j<7 s$, then $b_{i j}=0$.

If $7 s \leqslant j<8 s$, then

$$
b_{i j}=\left\{\begin{array}{l}
e_{4(j+m+s+1)+2} \otimes w_{4 j+3 \rightarrow 4(j+1)+2}, \quad i=3 s+(j+1)_{2 s} \\
0, \quad \text { otherwise. }
\end{array}\right.
$$

(II) Represent an arbitrary $t_{0} \in \mathbb{N}$ in the form $t_{0}=11 \ell_{0}+r_{0}$, where $0 \leqslant r_{0} \leqslant 10$. Then $\Omega^{t_{0}}\left(Y_{t}^{(13)}\right)$ is a $\Omega^{r_{0}}\left(Y_{t}^{(13)}\right)$, whose left components twisted by $\sigma^{\ell_{0}}$.

Proposition 30 (Translates for the case 14). (I) Let $r_{0} \in \mathbb{N}, r_{0}<11$. $r_{0}$-translates of the elements $Y_{t}^{(14)}$ are described by the following way.

(1) If $r_{0}=0$, then $\Omega^{0}\left(Y_{t}^{(14)}\right)$ is described with $(9 s \times 6 s)$-matrix with one nonzero element that is of the following form:

$$
b_{5 s, 7 s}=\kappa^{\ell}\left(\alpha_{3(j+m+3)}\right) w_{4(j+m)+3 \rightarrow 4(j+m+1)+3} \otimes e_{4 j+3} .
$$

(2) If $r_{0}=1$, then $\Omega^{1}\left(Y_{t}^{(14)}\right)$ is described with $(8 s \times 7 s)$-matrix with the following two nonzero elements:

$$
\begin{aligned}
& b_{j+2 s, 2 s+(3)_{s}}=-\kappa^{\ell}\left(\alpha_{3(j+m+3)}\right) w_{4(j+m)+3 \rightarrow 4(j+m+1)+3} \otimes w_{4 j+1 \rightarrow 4 j+2} ; \\
& b_{j+2 s, 3 s+(3)_{s}}=-\kappa^{\ell}\left(\alpha_{3(j+m+3)}\right) w_{4(j+m)+3 \rightarrow 4(j+m+1)+3} \otimes w_{4 j+1 \rightarrow 4 j+2} .
\end{aligned}
$$

(3) If $r_{0}=2$, then $\Omega^{2}\left(Y_{t}^{(14)}\right)$ is described with $(6 s \times 6 s)$-matrix with one nonzero element that is of the following form:

$$
b_{j,(3)_{s}}=-f_{2}(s, 1) \kappa^{\ell}\left(\alpha_{3(j+m+3)}\right) w_{4(j+m)-1 \rightarrow 4(j+m)+3} \otimes e_{4 j} .
$$

(4) If $r_{0}=3$, then $\Omega^{3}\left(Y_{t}^{(14)}\right)$ is described with $(7 s \times 8 s)$-matrix with one nonzero element that is of the following form:

$$
b_{(j+1)_{s},(2)_{s}}=-f_{2}(s, 1) \kappa^{\ell}\left(\alpha_{3(j+m+3)}\right) w_{4(j+m+1+s f(s, 1))+2 \rightarrow 4(j+m+1)+3} \otimes w_{4 j \rightarrow 4(j+1)} .
$$

(5) If $r_{0}=4$, then $\Omega^{4}\left(Y_{t}^{(14)}\right)$ is described with $(6 s \times 9 s)$-matrix with one nonzero element that is of the following form:

$$
b_{s+(j+1)_{s},(2)_{s}}=-f_{2}(s, 1) \kappa^{\ell}\left(\alpha_{3(j+m+3)}\right) e_{4(j+m+1)} \otimes w_{4 j \rightarrow 4(j+1)} .
$$


HOCHSCHILD COHOMOLOGY RING FOR SELF-INJECTIVE ALGEBRAS OF TREE CLASS E. II. 107

(6) If $r_{0}=5$, then $\Omega^{5}\left(Y_{t}^{(14)}\right)$ is described with $(6 s \times 8 s)$-matrix with one nonzero element that is of the following form:

$$
b_{(j+1)_{s},(2)_{s}}=\kappa^{\ell+1}\left(\alpha_{3(j+m-1)}\right) w_{4(j+m+1+s f(s, 1))+1 \rightarrow 4(j+m+2)} \otimes w_{4 j \rightarrow 4(j+1)} .
$$

(7) If $r_{0}=6$, then $\Omega^{6}\left(Y_{t}^{(14)}\right)$ is described with $(7 s \times 9 s)$-matrix with one nonzero element that is of the following form:

$$
b_{(j+1)_{s}, s+2-3 f(s, 1)}=-w_{4(j+m+1) \rightarrow 4(j+m+s+1)+1} \otimes w_{4 j \rightarrow 4(j+1)} .
$$

(8) If $r_{0}=7$, then $\Omega^{7}\left(Y_{t}^{(14)}\right)$ is described with $(6 s \times 8 s)$-matrix with one nonzero element that is of the following form:

$$
b_{(j+1)_{s},(2)_{s}}=-\kappa^{\ell+1}\left(\alpha_{3(j+m-1)}\right) w_{4(j+m+1+s f(s, 1))+2 \rightarrow 4(j+m+1)+3} \otimes w_{4 j \rightarrow 4(j+1)} .
$$

(9) If $r_{0}=8$, then $\Omega^{8}\left(Y_{t}^{(14)}\right)$ is described with $(8 s \times 6 s)$-matrix with one nonzero element that is of the following form:

$$
b_{(j+1)_{s}, s+2-3 f(s, 1)}=-f_{2}(s, 1) w_{4(j+m)+3 \rightarrow 4(j+m+s+1)+2} \otimes w_{4 j \rightarrow 4(j+1)} .
$$

(10) If $r_{0}=9$, then $\Omega^{9}\left(Y_{t}^{(14)}\right)$ is described with $(9 s \times 7 s)$-matrix with one nonzero element that is of the following form:

$$
b_{j,(2)_{s}}=\kappa^{\ell+1}\left(\alpha_{3(j+m-1)}\right) w_{4(j+m)+3 \rightarrow 4(j+m+1)+3} \otimes e_{4 j} .
$$

(11) If $r_{0}=10$, then $\Omega^{10}\left(Y_{t}^{(14)}\right)$ is described with $(8 s \times 6 s)$-matrix with one nonzero element that is of the following form:

$$
b_{j+s-f(s, 1), 4 s+1}=-f_{2}(s, 1) \kappa^{\ell+1}\left(\alpha_{3(j+m-1)}\right) w_{4(j+m)+3 \rightarrow 4(j+m+1)+3} \otimes w_{4 j+2 \rightarrow 4 j+3} .
$$

(II) Represent an arbitrary $t_{0} \in \mathbb{N}$ in the form $t_{0}=11 \ell_{0}+r_{0}$, where $0 \leqslant r_{0} \leqslant 10$. Then $\Omega^{t_{0}}\left(Y_{t}^{(14)}\right)$ is a $\Omega^{r_{0}}\left(Y_{t}^{(14)}\right)$, whose left components twisted by $\sigma^{\ell_{0}}$.

Proposition 31 (Translates for the case 15). (I) Let $r_{0} \in \mathbb{N}, r_{0}<11$. $r_{0}$-translates of the elements $Y_{t}^{(15)}$ are described by the following way.

(1) If $r_{0}=0$, then $\Omega^{0}\left(Y_{t}^{(15)}\right)$ is described with $(9 s \times 6 s)$-matrix with the following elements $b_{i j}$ : If $0 \leqslant j<s$, then

$$
b_{i j}=\left\{\begin{array}{l}
\kappa^{\ell}\left(\alpha_{3(j+m+3)}\right) e_{4(j+m)} \otimes e_{4 j}, \quad i=j \\
0, \quad \text { otherwise. }
\end{array}\right.
$$

If $s \leqslant j<2 s$, then $b_{i j}=0$.

If $2 s \leqslant j<3 s$, then

$$
b_{i j}=\left\{\begin{array}{l}
w_{4(j+m)+1 \rightarrow 4(j+m)+2} \otimes e_{4 j+1}, \quad i=j-s \\
0, \quad \text { otherwise. }
\end{array}\right.
$$

If $3 s \leqslant j<4 s$, then $b_{i j}=0$.

If $4 s \leqslant j<5 s$, then

$$
b_{i j}=\left\{\begin{array}{l}
w_{4(j+m+s)+1 \rightarrow 4(j+m+s)+2} \otimes e_{4(j+s)+1}, \quad i=j-2 s \\
0, \quad \text { otherwise. }
\end{array}\right.
$$

If $5 s \leqslant j<7 s$, then $b_{i j}=0$. 
If $7 s \leqslant j<8 s$, then

$$
b_{i j}=\left\{\begin{array}{l}
\kappa^{\ell}\left(\alpha_{3(j+m+3)}\right) e_{4(j+m)+3} \otimes e_{4 j+3}, \quad i=j-2 s \\
0, \quad \text { otherwise. }
\end{array}\right.
$$

If $8 s \leqslant j<9 s$, then $b_{i j}=0$.

(2) If $r_{0}=1$, then $\Omega^{1}\left(Y_{t}^{(15)}\right)$ is described with $(8 s \times 7 s)$-matrix with the following elements $b_{i j}$ : If $0 \leqslant j<2 s$, then

$$
b_{i j}=\left\{\begin{array}{l}
w_{4(j+m+s)+1 \rightarrow 4(j+m+s)+2} \otimes e_{4 j}, \quad i=(j+s)_{2 s} \\
0, \quad \text { otherwise. }
\end{array}\right.
$$

If $2 s \leqslant j<4 s$, then

$$
b_{i j}=\left\{\begin{array}{l}
\kappa_{1} w_{4(j+m)+2 \rightarrow 4(j+m)+3} \otimes e_{4 j+1}, \quad i=j \\
\kappa_{1} e_{4(j+m)+3} \otimes w_{4 j+1 \rightarrow 4 j+2}, \quad i=j+2 s \\
0, \quad \text { otherwise }
\end{array}\right.
$$

where $\kappa_{1}=-\kappa^{\ell}\left(\alpha_{3(j+m+3)}\right)$.

If $4 s \leqslant j<5 s$, then $b_{i j}=0$.

If $5 s \leqslant j<6 s$, then

$$
b_{i j}=\left\{\begin{array}{l}
-\kappa^{\ell}\left(\alpha_{3(j+m+4)}\right) e_{4(j+m+1)} \otimes w_{4 j+2 \rightarrow 4 j+3}, \quad i=j+s \\
0, \quad \text { otherwise. }
\end{array}\right.
$$

If $6 s \leqslant j<7 s$, then

$$
b_{i j}=\left\{\begin{array}{l}
w_{4(j+m+1) \rightarrow 4(j+m+s+1)+1} \otimes e_{4 j+3}, \quad i=j ; \\
0, \quad \text { otherwise. }
\end{array}\right.
$$

If $7 s \leqslant j<8 s$, then $b_{i j}=0$.

(3) If $r_{0}=2$, then $\Omega^{2}\left(Y_{t}^{(15)}\right)$ is described with $(6 s \times 6 s)$-matrix with the following elements $b_{i j}$ : If $0 \leqslant j<s$, then

$$
b_{i j}=\left\{\begin{array}{l}
-\kappa^{\ell}\left(\alpha_{3(j+m+2)}\right) e_{4(j+m)-1} \otimes e_{4 j}, \quad i=j \\
0, \quad \text { otherwise. }
\end{array}\right.
$$

If $s \leqslant j<3 s$, then

$$
b_{i j}=\left\{\begin{array}{l}
-w_{4(j+m)+1 \rightarrow 4(j+m)+2} \otimes w_{4(j+s)+1 \rightarrow 4(j+s)+2}, \quad i=j+2 s \\
0, \quad \text { otherwise. }
\end{array}\right.
$$

If $3 s \leqslant j<5 s$, then $b_{i j}=0$.

If $5 s \leqslant j<6 s$, then

$$
b_{i j}=\left\{\begin{array}{l}
\kappa^{\ell}\left(\alpha_{3(j+m+4)}\right) e_{4(j+m+1)} \otimes e_{4 j+3}, \quad i=j \\
0, \quad \text { otherwise }
\end{array}\right.
$$


HOCHSCHILD COHOMOLOGY RING FOR SELF-INJECTIVE ALGEBRAS OF TREE CLASS E6. II. 109 (4) If $r_{0}=3$, then $\Omega^{3}\left(Y_{t}^{(15)}\right)$ is described with $(7 s \times 8 s)$-matrix with the following elements $b_{i j}$ : If $0 \leqslant j<s$, then

$$
b_{i j}=\left\{\begin{array}{l}
-\kappa^{\ell}\left(\alpha_{3(j+m+3)}\right) w_{4(j+m)+2 \rightarrow 4(j+m)+3} \otimes e_{4 j}, \quad i=j \\
-\kappa^{\ell}\left(\alpha_{3(j+m+3)}\right) e_{4(j+m)+3} \otimes w_{4 j \rightarrow 4 j+1}, \quad i=j+2 s \\
0, \quad \text { otherwise. }
\end{array}\right.
$$

If $s \leqslant j<3 s$, then

$$
b_{i j}=\left\{\begin{array}{l}
-\kappa^{\ell}\left(\alpha_{3(j+m+4)}\right) e_{4(j+m+1)} \otimes w_{4(j+s)+1 \rightarrow 4(j+s)+2}, \quad i=j+3 s \\
0, \quad \text { otherwise. }
\end{array}\right.
$$

If $3 s \leqslant j<5 s$, then

$$
b_{i j}=\left\{\begin{array}{l}
w_{4(j+m+1) \rightarrow 4(j+m+s+1)+1} \otimes e_{4(j+s)+2}, \quad i=j+s \\
0, \quad \text { otherwise. }
\end{array}\right.
$$

If $5 s \leqslant j<7 s$, then

$$
b_{i j}=\left\{\begin{array}{l}
w_{4(j+m+1)+1 \rightarrow 4(j+m+1)+2} \otimes e_{4 j+3}, \quad i=6 s+(j)_{2 s} ; \\
0, \quad \text { otherwise. }
\end{array}\right.
$$

(5) If $r_{0}=4$, then $\Omega^{4}\left(Y_{t}^{(15)}\right)$ is described with $(6 s \times 9 s)$-matrix with the following elements $b_{i j}$ : If $0 \leqslant j<s$, then

$$
b_{i j}=\left\{\begin{array}{l}
-\kappa^{\ell}\left(\alpha_{3(j+m+3)}\right) e_{4(j+m)} \otimes e_{4 j}, \quad i=j+s \\
0, \quad \text { otherwise. }
\end{array}\right.
$$

If $s \leqslant j<3 s$, then $b_{i j}=0$.

If $3 s \leqslant j<5 s$, then

$$
b_{i j}=\left\{\begin{array}{l}
w_{4(j+m+s)+1 \rightarrow 4(j+m+s)+2} \otimes e_{4(j+s)+2}, \quad i=j+2 s-s f_{0}(j, 4 s) \\
0, \quad \text { otherwise. }
\end{array}\right.
$$

If $5 s \leqslant j<6 s$, then

$$
b_{i j}=\left\{\begin{array}{l}
-\kappa^{\ell}\left(\alpha_{3(j+m+3)}\right) e_{4(j+m)+3} \otimes e_{4 j+3}, \quad i=j+3 s \\
0, \quad \text { otherwise. }
\end{array}\right.
$$

(6) If $r_{0}=5$, then $\Omega^{5}\left(Y_{t}^{(15)}\right)$ is described with $(6 s \times 8 s)$-matrix with the following elements $b_{i j}$ : If $0 \leqslant j<s$, then

$$
b_{i j}=\left\{\begin{array}{l}
\kappa_{1} w_{4(j+m+s)+1 \rightarrow 4(j+m+1)} \otimes e_{4 j}, \quad i=j+s \\
\kappa_{1} e_{4(j+m+1)} \otimes w_{4 j \rightarrow 4 j+1}, \quad i=j+2 s \\
-\kappa_{1} w_{4(j+m)+3 \rightarrow 4(j+m+1)} \otimes w_{4 j \rightarrow 4 j+2}, \quad i=j+4 s \\
0, \quad \text { otherwise }
\end{array}\right.
$$


where $\kappa_{1}=-\kappa^{\ell}\left(\alpha_{3(j+m+4)}\right)$.

If $s \leqslant j<3 s$, then

$$
b_{i j}=\left\{\begin{array}{l}
e_{4(j+m+s+1)+1} \otimes w_{4(j+s)+1 \rightarrow 4(j+1)}, \quad i=(j+s+1)_{2 s} \\
-w_{4(j+m)+3 \rightarrow 4(j+m+s+1)+1} \otimes w_{4(j+s)+1 \rightarrow 4(j+s)+2}, \quad i=j+3 s \\
0, \quad \text { otherwise. }
\end{array}\right.
$$

If $3 s \leqslant j<5 s$, then

$$
b_{i j}=\left\{\begin{array}{l}
-w_{4(j+m+s+1)+1 \rightarrow 4(j+m+s+1)+2} \otimes w_{4(j+s)+2 \rightarrow 4(j+1)}, \quad i=(j+s+1)_{2 s} \\
w_{4(j+m)+3 \rightarrow 4(j+m+s+1)+2} \otimes e_{4(j+s)+2}, \quad i=j+s \\
0, \quad \text { otherwise. }
\end{array}\right.
$$

If $5 s \leqslant j<6 s$, then

$$
b_{i j}=\left\{\begin{array}{l}
-\kappa_{1} w_{4(j+m+s+1)+1 \rightarrow 4(j+m+1)+3} \otimes w_{4 j+3 \rightarrow 4(j+1)}, \quad i=(j+s+1)_{2 s} ; \\
\kappa_{1} e_{4(j+m+1)+3} \otimes w_{4 j+3 \rightarrow 4(j+1)+2}, \quad i=4 s+(j+1)_{2 s} \\
-\kappa_{1} w_{4(j+m+s+1)+2 \rightarrow 4(j+m+1)+3} \otimes e_{4 j+3}, \quad i=j+s \\
0, \quad \text { otherwise, }
\end{array}\right.
$$

where $\kappa_{1}=\kappa^{\ell}\left(\alpha_{3(j+m+4)}\right)$.

(7) If $r_{0}=6$, then $\Omega^{6}\left(Y_{t}^{(15)}\right)$ is described with $(7 s \times 9 s)$-matrix with the following elements $b_{i j}$ : If $0 \leqslant j<s$, then

$$
b_{i j}=\left\{\begin{array}{l}
e_{4(j+m)+1} \otimes w_{4 j \rightarrow 4 j+1}, \quad i=j+s \\
0, \quad \text { otherwise }
\end{array}\right.
$$

If $s \leqslant j<2 s$, then

$$
b_{i j}=\left\{\begin{array}{l}
-w_{4(j+m) \rightarrow 4(j+m)+1} \otimes e_{4 j}, \quad i=j-s \\
e_{4(j+m)+1} \otimes w_{4 j \rightarrow 4 j+1}, \quad i=j+2 s \\
0, \quad \text { otherwise. }
\end{array}\right.
$$

If $2 s \leqslant j<4 s$, then $b_{i j}=0$.

If $4 s \leqslant j<5 s$, then

$$
b_{i j}=\left\{\begin{array}{l}
\kappa^{\ell}\left(\alpha_{3(j+m+3)}\right) w_{4(j+m)+2 \rightarrow 4(j+m)+3} \otimes e_{4 j+2}, \quad i=j+s \\
0, \quad \text { otherwise. }
\end{array}\right.
$$

If $5 s \leqslant j<6 s$, then

$$
b_{i j}=\left\{\begin{array}{l}
-\kappa_{1} w_{4(j+m)+2 \rightarrow 4(j+m)+3} \otimes e_{4 j+2}, \quad i=j+s \\
\kappa_{1} e_{4(j+m)+3} \otimes w_{4 j+2 \rightarrow 4 j+3}, \quad i=j+2 s \\
0, \quad \text { otherwise }
\end{array}\right.
$$

where $\kappa_{1}=\kappa^{\ell}\left(\alpha_{3(j+m+3)}\right)$. 
HOCHSCHILD COHOMOLOGY RING FOR SELF-INJECTIVE ALGEBRAS OF TREE CLASS $E_{6}$. II. 111

If $6 s \leqslant j<7 s$, then

$$
b_{i j}=\left\{\begin{array}{l}
-f_{2}(j, 7 s-1) \kappa_{1} e_{4(j+m+1)} \otimes e_{4 j+3}, \quad i=j+2 s \\
\kappa_{1} e_{4(j+m+1)} \otimes w_{4 j+3 \rightarrow 4(j+1)}, \quad i=(j+1)_{s}, j<7 s-1 \\
0, \quad \text { otherwise, }
\end{array}\right.
$$

where $\kappa_{1}=\kappa^{\ell}\left(\alpha_{3(j+m+4)}\right)$.

(8) If $r_{0}=7$, then $\Omega^{7}\left(Y_{t}^{(15)}\right)$ is described with $(6 s \times 8 s)$-matrix with the following elements $b_{i j}$ :

If $0 \leqslant j<s$, then

$$
b_{i j}=\left\{\begin{array}{l}
\kappa_{1} w_{4(j+m+s)+2 \rightarrow 4(j+m)+3} \otimes e_{4 j}, \quad i=j+s \\
-\kappa_{1} e_{4(j+m)+3} \otimes w_{4 j \rightarrow 4 j+1}, \quad i=j+2 s \\
0, \quad \text { otherwise }
\end{array}\right.
$$

where $\kappa_{1}=\kappa^{\ell}\left(\alpha_{3(j+m+3)}\right)$.

If $s \leqslant j<3 s$, then

$$
b_{i j}=\left\{\begin{array}{l}
e_{4(j+m+s+1)+2} \otimes w_{4(j+s)+1 \rightarrow 4(j+1)}, \quad i=(j+s+1)_{2 s} ; \\
-w_{4(j+m+1) \rightarrow 4(j+m+s+1)+2} \otimes w_{4(j+s)+1 \rightarrow 4(j+s)+2}, \quad i=j+3 s \\
0, \quad \text { otherwise. }
\end{array}\right.
$$

If $3 s \leqslant j<5 s$, then

$$
b_{i j}=\left\{\begin{array}{l}
-w_{4(j+m+1) \rightarrow 4(j+m+1)+1} \otimes e_{4(j+s)+2}, \quad i=j+s \\
0, \quad \text { otherwise }
\end{array}\right.
$$

If $5 s \leqslant j<6 s$, then

$$
b_{i j}=\left\{\begin{array}{l}
-\kappa_{1} e_{4(j+m+2)} \otimes w_{4 j+3 \rightarrow 4(j+s+1)+2}, \quad i=4 s+(j+s+1)_{2 s} ; \\
\kappa_{1} w_{4(j+m+s+1)+1 \rightarrow 4(j+m+2)} \otimes e_{4 j+3}, \quad i=j+s ; \\
\kappa_{1} w_{4(j+m+s+1)+2 \rightarrow 4(j+m+2)} \otimes w_{4 j+3 \rightarrow 4(j+1)}, \quad i=s+(j+1)_{s}, j=6 s-1 ; \\
-\kappa_{1} w_{4(j+m+1)+3 \rightarrow 4(j+m+2)} \otimes w_{4 j+3 \rightarrow 4(j+1)+1}, \quad i=2 s+(j+1)_{s}, j=6 s-1 ; \\
0, \quad \text { otherwise, }
\end{array}\right.
$$

where $\kappa_{1}=\kappa^{\ell+1}\left(\alpha_{3(j+m-1)}\right)$.

(9) If $r_{0}=8$, then $\Omega^{8}\left(Y_{t}^{(15)}\right)$ is described with $(8 s \times 6 s)$-matrix with the following elements $b_{i j}$ :

If $0 \leqslant j<s$, then

$$
b_{i j}=\left\{\begin{array}{l}
e_{4(j+m)+2} \otimes w_{4 j \rightarrow 4 j+1}, \quad i=j+s \\
w_{4(j+m)+1 \rightarrow 4(j+m)+2} \otimes w_{4 j \rightarrow 4(j+s)+2}, \quad i=j+4 s \\
0, \quad \text { otherwise. }
\end{array}\right.
$$


If $s \leqslant j<2 s$, then

$$
b_{i j}=\left\{\begin{array}{l}
-w_{4(j+m)-1 \rightarrow 4(j+m)+2} \otimes e_{4 j}, \quad i=j-s \\
-e_{4(j+m)+2} \otimes w_{4 j \rightarrow 4 j+1}, \quad i=j+s ; \\
-w_{4(j+m)+1 \rightarrow 4(j+m)+2} \otimes w_{4 j \rightarrow 4(j+s)+2}, \quad i=j+2 s \\
0, \quad \text { otherwise. }
\end{array}\right.
$$

If $2 s \leqslant j<4 s$, then

$$
b_{i j}=\left\{\begin{array}{l}
\kappa^{\ell}\left(\alpha_{3(j+m+3)}\right) e_{4(j+m)+3} \otimes w_{4 j+1 \rightarrow 4(j+1)}, \quad i=(j+1)_{s}, j<3 s-1 \text { or } j=4 s-1 ; \\
0, \quad \text { otherwise. }
\end{array}\right.
$$

If $4 s \leqslant j<6 s$, then

$$
b_{i j}=\left\{\begin{array}{l}
\kappa_{1} w_{4(j+m+s)+1 \rightarrow 4(j+m+1)} \otimes e_{4 j+2}, \quad i=j-s \\
-\kappa_{1} e_{4(j+m+1)} \otimes w_{4 j+2 \rightarrow 4 j+3}, \quad i=j+s, j<5 s \\
0, \quad \text { otherwise, }
\end{array}\right.
$$

where $\kappa_{1}=-\kappa^{\ell+1}\left(\alpha_{3(j+m-2)}\right)$.

If $6 s \leqslant j<7 s$, then

$$
b_{i j}=\left\{\begin{array}{l}
-w_{4(j+m+1) \rightarrow 4(j+m+1)+1} \otimes e_{4 j+3}, \quad i=j-s \\
0, \quad \text { otherwise. }
\end{array}\right.
$$

If $7 s \leqslant j<8 s$, then $b_{i j}=0$.

(10) If $r_{0}=9$, then $\Omega^{9}\left(Y_{t}^{(15)}\right)$ is described with $(9 s \times 7 s)$-matrix with the following elements $b_{i j}$ :

If $0 \leqslant j<s$, then

$$
b_{i j}=\left\{\begin{array}{l}
\kappa^{\ell+1}\left(\alpha_{3(j+m-3)}\right) e_{4(j+m)+3} \otimes e_{4 j}, \quad i=j \\
0, \quad \text { otherwise. }
\end{array}\right.
$$

If $s \leqslant j<2 s$, then

$$
b_{i j}=\left\{\begin{array}{l}
-\kappa^{\ell+1}\left(\alpha_{3(j+m-2)}\right) e_{4(j+m+1)} \otimes w_{4 j \rightarrow 4(j+s)+1}, \quad i=j ; \\
0, \quad \text { otherwise. }
\end{array}\right.
$$

If $2 s \leqslant j<4 s$, then

$$
b_{i j}=\left\{\begin{array}{l}
-w_{4(j+m+1) \rightarrow 4(j+m+s+1)+1} \otimes e_{4 j+1}, \quad i=j-s \\
0, \quad \text { otherwise. }
\end{array}\right.
$$

If $4 s \leqslant j<5 s$, then $b_{i j}=0$.

If $5 s \leqslant j<6 s$, then

$$
b_{i j}=\left\{\begin{array}{l}
-w_{4(j+m+1)+1 \rightarrow 4(j+m+1)+2} \otimes e_{4(j+s)+2}, \quad i=j-2 s \\
0, \quad \text { otherwise. }
\end{array}\right.
$$


HOCHSCHILD COHOMOLOGY RING FOR SELF-INJECTIVE ALGEBRAS OF TREE CLASS E . II. 113

If $6 s \leqslant j<7 s$, then $b_{i j}=0$.

If $7 s \leqslant j<8 s$, then

$$
b_{i j}=\left\{\begin{array}{l}
-w_{4(j+m+s+1)+1 \rightarrow 4(j+m+s+1)+2} \otimes e_{4 j+2}, \quad i=j-3 s \\
0, \quad \text { otherwise. }
\end{array}\right.
$$

If $8 s \leqslant j<9 s$, then

$$
b_{i j}=\left\{\begin{array}{l}
\kappa_{1} w_{4(j+m+1)+2 \rightarrow 4(j+m+1)+3} \otimes e_{4 j+3}, \quad i=j-3 s \\
-\kappa_{1} e_{4(j+m+1)+3} \otimes w_{4 j+3 \rightarrow 4(j+1)}, \quad i=(j+1)_{s}, \quad j=9 s-1 \\
0, \quad \text { otherwise }
\end{array}\right.
$$

where $\kappa_{1}=-\kappa^{\ell+1}\left(\alpha_{3(j+m-2)}\right)$.

(11) If $r_{0}=10$, then $\Omega^{10}\left(Y_{t}^{(15)}\right)$ is described with $(8 s \times 6 s)$-matrix with the following elements $b_{i j}$ :

If $0 \leqslant j<s$, then

$$
b_{i j}=\left\{\begin{array}{l}
-w_{4(j+m) \rightarrow 4(j+m)+1} \otimes e_{4 j}, \quad i=j ; \\
0, \quad \text { otherwise. }
\end{array}\right.
$$

If $s \leqslant j<2 s$, then $b_{i j}=0$.

If $2 s \leqslant j<4 s$, then

$$
b_{i j}=\left\{\begin{array}{l}
\kappa_{1} w_{4(j+m+s)+1 \rightarrow 4(j+m+1)} \otimes e_{4 j+1}, \quad i=j-s \\
\kappa_{1} e_{4(j+m+1)} \otimes w_{4 j+1 \rightarrow 4(j+1)}, \quad i=(j+1)_{s}, j<3 s-1 \text { or } j=4 s-1 \\
0, \quad \text { otherwise }
\end{array}\right.
$$

where $\kappa_{1}=-f_{1}(j, 3 s) \kappa^{\ell}\left(\alpha_{3(j+m-2)}\right)$.

If $4 s \leqslant j<6 s$, then

$$
b_{i j}=\left\{\begin{array}{l}
\kappa_{1} w_{4(j+m+s)+2 \rightarrow 4(j+m)+3} \otimes e_{4 j+2}, \quad i=j-s \\
\kappa_{1} e_{4(j+m)+3} \otimes w_{4 j+2 \rightarrow 4 j+3}, \quad i=j+s, j<5 s \\
0, \quad \text { otherwise }
\end{array}\right.
$$

where $\kappa_{1}=\kappa^{\ell+1}\left(\alpha_{3(j+m-3)}\right)$.

If $6 s \leqslant j<7 s$, then

$$
b_{i j}=\left\{\begin{array}{l}
w_{4(j+m+1)+1 \rightarrow 4(j+m+1)+2} \otimes w_{4 j+3 \rightarrow 4(j+s+1)+1}, \quad i=s+(j+s+1)_{2 s} ; \\
-w_{4(j+m)+3 \rightarrow 4(j+m+1)+2} \otimes e_{4 j+3}, \quad i=j-s \\
0, \quad \text { otherwise. }
\end{array}\right.
$$

If $7 s \leqslant j<8 s$, then

$$
b_{i j}=\left\{\begin{array}{l}
-w_{4(j+m+1)+1 \rightarrow 4(j+m+1)+2} \otimes w_{4 j+3 \rightarrow 4(j+s+1)+1}, \quad i=s+(j+s+1)_{2 s} \\
0, \quad \text { otherwise. }
\end{array}\right.
$$


(II) Represent an arbitrary $t_{0} \in \mathbb{N}$ in the form $t_{0}=11 \ell_{0}+r_{0}$, where $0 \leqslant r_{0} \leqslant 10$. Then $\Omega^{t_{0}}\left(Y_{t}^{(15)}\right)$ is a $\Omega^{r_{0}}\left(Y_{t}^{(15)}\right)$, whose left components twisted by $\sigma^{\ell_{0}}$.

Proposition 32 (Translates for the case 16). (I) Let $r_{0} \in \mathbb{N}, r_{0}<11$. $r_{0}$-translates of the elements $Y_{t}^{(16)}$ are described by the following way.

(1) If $r_{0}=0$, then $\Omega^{0}\left(Y_{t}^{(16)}\right)$ is described with $(8 s \times 6 s)$-matrix with the following two nonzero elements:

$$
\begin{gathered}
b_{0,0}=w_{4(j+m) \rightarrow 4(j+m)+2} \otimes e_{4 j} ; \\
b_{s, 2 s}=w_{4(j+m)+1 \rightarrow 4(j+m)+3} \otimes e_{4 j+1} .
\end{gathered}
$$

(2) If $r_{0}=1$, then $\Omega^{1}\left(Y_{t}^{(16)}\right)$ is described with $(6 s \times 7 s)$-matrix with the following nonzero elements:

$$
\begin{gathered}
b_{j, 0}=w_{4(j+m)+1 \rightarrow 4(j+m)+3} \otimes e_{4 j} ; \\
b_{(j+1)_{s}, 3 s-1}=w_{4(j+m+s+1)+1 \rightarrow 4(j+m+s+1)+2} \otimes w_{4(j+s)+1 \rightarrow 4(j+1)} ; \\
b_{2 s+(j+1)_{s}, 3 s-1}=e_{4(j+m+s+1)+2} \otimes w_{4(j+s)+1 \rightarrow 4(j+s+1)+1} ; \\
b_{(j+1)_{s}, 6 s-1}=w_{4(j+m+1)+1 \rightarrow 4(j+m+2)} \otimes w_{4 j+3 \rightarrow 4(j+1)} ; \\
b_{j+s, 6 s-1}=w_{4(j+m+1) \rightarrow 4(j+m+2)} \otimes e_{4 j+3} .
\end{gathered}
$$

(3) If $r_{0}=2$, then $\Omega^{2}\left(Y_{t}^{(16)}\right)$ is described with $(7 s \times 6 s)$-matrix with the following nonzero elements:

$$
\begin{aligned}
b_{(j+1)_{s}, 2 s-1} & =w_{4(j+m)+3 \rightarrow 4(j+m+1)} \otimes w_{4(j+s)+1 \rightarrow 4(j+1)} \\
b_{j, 2 s-1} & =w_{4(j+m+s)+2 \rightarrow 4(j+m+1)} \otimes e_{4(j+s)+1} \\
b_{j, 3 s-1} & =w_{4(j+m+s)+2 \rightarrow 4(j+m+1)} \otimes e_{4(j+s)+1} \\
b_{j, 4 s-1} & =w_{4(j+m)+1 \rightarrow 4(j+m+1)+1} \otimes e_{4(j+s)+2} .
\end{aligned}
$$

(4) If $r_{0}=3$, then $\Omega^{3}\left(Y_{t}^{(16)}\right)$ is described with $(6 s \times 8 s)$-matrix with the following nonzero elements:

$$
\begin{gathered}
b_{j+s, s-1}=w_{4(j+m+s)+2 \rightarrow 4(j+m+1)} \otimes e_{4 j} ; \\
b_{j+2 s, s-1}=w_{4(j+m)+3 \rightarrow 4(j+m+1)} \otimes w_{4 j \rightarrow 4 j+1} ; \\
b_{j+s, 2 s-1}=w_{4(j+m)+3 \rightarrow 4(j+m+1)+1} \otimes e_{4(j+s)+1} .
\end{gathered}
$$

(5) If $r_{0}=4$, then $\Omega^{4}\left(Y_{t}^{(16)}\right)$ is described with $(6 s \times 9 s)$-matrix with the following nonzero elements:

$$
\begin{gathered}
b_{(j+1)_{s},(s-2)_{s}}=w_{4(j+m)+3 \rightarrow 4(j+m+1)} \otimes w_{4 j \rightarrow 4(j+1)} ; \\
b_{s+(j+1)_{s},(s-2)_{s}}=e_{4(j+m+1)} \otimes w_{4 j \rightarrow 4(j+1)} ; \\
b_{(j+1)_{s}, 2 s-(2)_{s}}=w_{4(j+m)+3 \rightarrow 4(j+m+1)+1} \otimes w_{4(j+s)+1 \rightarrow 4(j+1)} ; \\
b_{s+(j+1)_{s}, 2 s-(2)_{s}}=w_{4(j+m+1) \rightarrow 4(j+m+1)+1} \otimes w_{4(j+s)+1 \rightarrow 4(j+1)} ; \\
b_{j+2 s+f(s, 1), 4 s-(2)_{s}}=w_{4(j+m)+2 \rightarrow 4(j+m+1)+2} \otimes e_{4(j+s)+2} .
\end{gathered}
$$

(6) If $r_{0}=5$, then $\Omega^{5}\left(Y_{t}^{(16)}\right)$ is described with $(7 s \times 8 s)$-matrix with the following two nonzero elements:

$$
\begin{gathered}
b_{(j+1)_{s}, 2 s-2}=e_{4(j+m+s+1)+1} \otimes w_{4 j \rightarrow 4(j+1)} ; \\
b_{j, 3 s-(2)_{s}}=w_{4(j+m+1) \rightarrow 4(j+m+s+1)+2} \otimes e_{4 j+1} .
\end{gathered}
$$

(7) If $r_{0}=6$, then $\Omega^{6}\left(Y_{t}^{(16)}\right)$ is described with $(6 s \times 9 s)$-matrix with the following nonzero elements:

$$
b_{j+s+2 f(s, 1),(s-2)_{s}}=w_{4(j+m+f(s, 1))+1 \rightarrow 4(j+m)+3} \otimes w_{4 j \rightarrow 4(j+f(s, 1))+1}
$$


HOCHSCHILD COHOMOLOGY RING FOR SELF-INJECTIVE ALGEBRAS OF TREE CLASS E $E_{6}$ II. 115

$$
\begin{gathered}
b_{(j+1)_{s}, 2 s-3+2 f(s, 1)}=w_{4(j+m+1) \rightarrow 4(j+m+1)+2} \otimes w_{4(j+s)+1 \rightarrow 4(j+1)} ; \\
b_{s+(j+1)_{s}, 6 s-3}=w_{4(j+m+s+1)+1 \rightarrow 4(j+m+2)} \otimes w_{4 j+3 \rightarrow 4(j+s+1)+1}, \quad s>1 .
\end{gathered}
$$

(8) If $r_{0}=7$, then $\Omega^{7}\left(Y_{t}^{(16)}\right)$ is described with $(8 s \times 8 s)$-matrix with the following nonzero elements:

$$
\begin{gathered}
b_{s+(j+1)_{s}-f(s, 1),(s-3)_{s}}=e_{4(j+m+s+1)+2} \otimes w_{4 j \rightarrow 4(j+1)} ; \\
b_{(j+1)_{s}+f(s, 1), 2 s+(s-3)_{s}}=w_{4(j+m+1)+2 \rightarrow 4(j+m+1)+3} \otimes w_{4 j+1 \rightarrow 4(j+1)} \\
b_{2 s+(j+1)_{s}+f(s, 1), 7 s+(s-3)_{s}}=w_{4(j+m+1)+3 \rightarrow 4(j+m+s+2)+1} \otimes w_{4 j+3 \rightarrow 4(j+s+1)+1} \\
b_{4 s+(j+1)_{s}+f(s, 1), 7 s+(s-3)_{s}}=w_{4(j+m+2) \rightarrow 4(j+m+s+2)+1} \otimes w_{4 j+3 \rightarrow 4(j+s+1)+2} \\
b_{5 s+(j+1)_{s}-f(s, 1), 7 s+(s-3)_{s}}=w_{4(j+m+2) \rightarrow 4(j+m+s+2)+1} \otimes w_{4 j+3 \rightarrow 4(j+1)+2} .
\end{gathered}
$$

(9) If $r_{0}=8$, then $\Omega^{8}\left(Y_{t}^{(16)}\right)$ is described with $(9 s \times 6 s)$-matrix with the following nonzero elements:

$$
\begin{gathered}
b_{(j+1)_{s},(s-3)_{s}}=e_{4(j+m)+3} \otimes w_{4 j \rightarrow 4(j+1)} ; \\
b_{(j+1)_{s}, s+(s-3)_{s}}=w_{4(j+m)+3 \rightarrow 4(j+m+1)} \otimes w_{4 j \rightarrow 4(j+1)} ; \\
b_{(j+1)_{s}, 2 s+(s-3)_{s}}=w_{4(j+m)+3 \rightarrow 4(j+m+1)+1} \otimes w_{4 j+1 \rightarrow 4(j+1)} ; \\
b_{s+(j+1)_{s}+f(s, 1), 5 s+(s-3)_{s}}=e_{4(j+m+s+1)+2} \otimes w_{4(j+s)+2 \rightarrow 4(j+s+1)+1} ; \\
b_{4 s+(j+1)_{s}-f(s, 1), 6 s+(s-3)_{s}}=e_{4(j+m+1)+1} \otimes w_{4(j+s)+2 \rightarrow 4(j+s+1)+2} ; \\
b_{(j+1)_{s}, 8 s+(s-3)_{s}}=w_{4(j+m)+3 \rightarrow 4(j+m+1)+3} \otimes w_{4 j+3 \rightarrow 4(j+1)} ; \\
b_{2 s+(j+1)_{s}-f(s, 1), 8 s+(s-3)_{s}}=w_{4(j+m+s+1)+2 \rightarrow 4(j+m+1)+3} \otimes w_{4 j+3 \rightarrow 4(j+s+1)+1} ; \\
b_{3 s+(j+1)_{s}+f(s, 1), 8 s+(s-3)_{s}}=w_{4(j+m+s+1)+1 \rightarrow 4(j+m+1)+3} \otimes w_{4 j+3 \rightarrow 4(j+1)+2} .
\end{gathered}
$$

(10) If $r_{0}=9$, then $\Omega^{9}\left(Y_{t}^{(16)}\right)$ is described with $(8 s \times 7 s)$-matrix with the following nonzero elements:

$$
\begin{gathered}
b_{(j+1)_{s}, 3 s-3+2 f(s, 1)}=w_{4(j+m+1)+3 \rightarrow 4(j+m+2)} \otimes w_{4 j+1 \rightarrow 4(j+1)} ; \\
b_{(j+1)_{s}, 5 s-3+2 f(s, 1)}=e_{4(j+m+1)+3} \otimes w_{4 j+2 \rightarrow 4(j+1)} ; \\
b_{(j+1)_{s}, 6 s-3+2 f(s, 1)}=e_{4(j+m+1)+3} \otimes w_{4 j+2 \rightarrow 4(j+1)} ; \\
b_{(j+1)_{s}, 7 s-3+2 f(s, 1)}=w_{4(j+m+1)+3 \rightarrow 4(j+m+s+2)+2} \otimes w_{4 j+3 \rightarrow 4(j+1)} .
\end{gathered}
$$

(11) If $r_{0}=10$, then $\Omega^{10}\left(Y_{t}^{(16)}\right)$ is described with $(9 s \times 6 s)$-matrix with the following nonzero elements:

$$
\begin{aligned}
& b_{(j+1)_{s},(s-3)_{s}}=e_{4(j+m+1)} \otimes w_{4 j \rightarrow 4(j+1)} ; \\
& b_{(j+1)_{s}, s+(s-3)_{s}}=w_{4(j+m+1) \rightarrow 4(j+m+1)+1} \otimes w_{4(j+s)+1 \rightarrow 4(j+1)} ; \\
& b_{s+(j+1)_{s}+f(s, 1), s+(s-3)_{s}}=e_{4(j+m+1)+1} \otimes w_{4(j+s)+1 \rightarrow 4(j+s+1)+1} \text {; } \\
& b_{j-2 s, 6 s+(s-3)_{s}}=w_{4(j+m)+2 \rightarrow 4(j+m+1)+2} \otimes e_{4(j+s)+2} ; \\
& b_{j-s, 6 s+(s-3)_{s}}=w_{4(j+m)+3 \rightarrow 4(j+m+1)+2} \otimes w_{4(j+s)+2 \rightarrow 4 j+3} \text {; } \\
& b_{j-2 s, 7 s+(s-3)_{s}}=w_{4(j+m)+3 \rightarrow 4(j+m+1)+3} \otimes e_{4 j+3} .
\end{aligned}
$$

(II) Represent an arbitrary $t_{0} \in \mathbb{N}$ in the form $t_{0}=11 \ell_{0}+r_{0}$, where $0 \leqslant r_{0} \leqslant 10$. Then $\Omega^{t_{0}}\left(Y_{t}^{(16)}\right)$ is a $\Omega^{r_{0}}\left(Y_{t}^{(16)}\right)$, whose left components twisted by $\sigma^{\ell_{0}}$. 
Proposition 33 (Translates for the case 17). (I) Let $r_{0} \in \mathbb{N}, r_{0}<11$. $r_{0}$-translates of the elements $Y_{t}^{(17)}$ are described by the following way.

(1) If $r_{0}=0$, then $\Omega^{0}\left(Y_{t}^{(17)}\right)$ is described with $(8 s \times 6 s)$-matrix with the following two nonzero elements:

$$
\begin{aligned}
& b_{0,0}=w_{4(j+m) \rightarrow 4(j+m+s)+2} \otimes e_{4 j} ; \\
& b_{0, s}=w_{4(j+m) \rightarrow 4(j+m+s)+2} \otimes e_{4 j} .
\end{aligned}
$$

(2) If $r_{0}=1$, then $\Omega^{1}\left(Y_{t}^{(17)}\right)$ is described with $(6 s \times 7 s)$-matrix with the following nonzero elements:

$$
\begin{gathered}
b_{s+(j+1)_{s}-f(s, 1), s+(1)_{s}}=-w_{4(j+m+1)+1 \rightarrow 4(j+m+1)+2} \otimes w_{4(j+s)+1 \rightarrow 4(j+1)} \\
b_{(j+1)_{s}+f(s, 1), 2 s+(1)_{s}}=-w_{4(j+m+1)+1 \rightarrow 4(j+m+1)+2} \otimes w_{4(j+s)+1 \rightarrow 4(j+1)} \\
b_{j+s, 5 s+(1)_{s}}=-\kappa^{\ell}\left(\alpha_{3(j+m+5)}\right) w_{4(j+m+1) \rightarrow 4(j+m+2)} \otimes e_{4 j+3} .
\end{gathered}
$$

(3) If $r_{0}=2$, then $\Omega^{2}\left(Y_{t}^{(17)}\right)$ is described with $(7 s \times 6 s)$-matrix with the following nonzero elements:

$$
\begin{gathered}
b_{(j+1)_{s},(1)_{s}}=f_{2}(s, 1) \kappa^{\ell}\left(\alpha_{3(j+m+3)}\right) e_{4(j+m)+3} \otimes w_{4 j \rightarrow 4(j+1)} ; \\
b_{(j+1)_{s}, s+(1)_{s}}=-f_{2}(s, 1) \kappa^{\ell}\left(\alpha_{3(j+m+4)}\right) w_{4(j+m)+3 \rightarrow 4(j+m+1)} \otimes w_{4(j+s)+1 \rightarrow 4(j+1)} ; \\
b_{j, s+(1)_{s}}=-\kappa^{\ell}\left(\alpha_{3(j+m+4)}\right) w_{4(j+m+s)+2 \rightarrow 4(j+m+1)} \otimes e_{4(j+s)+1} ; \\
b_{(j+1)_{s}, 2 s+(1)_{s}}=f_{2}(s, 1) \kappa^{\ell}\left(\alpha_{3(j+m+4)}\right) w_{4(j+m)+3 \rightarrow 4(j+m+1)} \otimes w_{4(j+s)+1 \rightarrow 4(j+1)} ; \\
b_{j, 2 s+(1)_{s}}=-\kappa^{\ell}\left(\alpha_{3(j+m+4)}\right) w_{4(j+m+s)+2 \rightarrow 4(j+m+1)} \otimes e_{4(j+s)+1} .
\end{gathered}
$$

(4) If $r_{0}=3$, then $\Omega^{3}\left(Y_{t}^{(17)}\right)$ is described with $(6 s \times 8 s)$-matrix with the following nonzero elements:

$$
\begin{gathered}
b_{j+s,(1)_{s}}=\kappa^{\ell}\left(\alpha_{3(j+m+4)}\right) w_{4(j+m+s)+2 \rightarrow 4(j+m+1)} \otimes e_{4 j} ; \\
b_{j+2 s,(1)_{s}}=-\kappa^{\ell}\left(\alpha_{3(j+m+4)}\right) w_{4(j+m)+3 \rightarrow 4(j+m+1)} \otimes w_{4 j \rightarrow 4 j+1} ; \\
b_{j+s, s+(1)_{s}}=-w_{4(j+m)+3 \rightarrow 4(j+m+s+1)+1} \otimes e_{4(j+s)+1} ; \\
b_{j+s, 2 s+(1)_{s}}=-w_{4(j+m)+3 \rightarrow 4(j+m+s+1)+1} \otimes e_{4(j+s)+1} .
\end{gathered}
$$

(5) If $r_{0}=4$, then $\Omega^{4}\left(Y_{t}^{(17)}\right)$ is described with $(6 s \times 9 s)$-matrix with the following nonzero elements:

$$
\begin{gathered}
b_{(j+1)_{s}, 0}=-f_{2}(s, 1) \kappa^{\ell}\left(\alpha_{3(j+m+4)}\right) w_{4(j+m)+3 \rightarrow 4(j+m+1)} \otimes w_{4 j \rightarrow 4(j+1)} \\
b_{s+(j+1)_{s}, 0}=f_{2}(s, 1) \kappa^{\ell}\left(\alpha_{3(j+m+4)}\right) e_{4(j+m+1)} \otimes w_{4 j \rightarrow 4(j+1)} \\
b_{(j+1)_{s}, 2 s-f(s, 1)}=w_{4(j+m)+3 \rightarrow 4(j+m+s+1)+1} \otimes w_{4(j+s)+1 \rightarrow 4(j+1)} \\
b_{(j+1)_{s}, 4 s-f(s, 1)}=-w_{4(j+m)+3 \rightarrow 4(j+m+s+1)+2} \otimes w_{4(j+s)+2 \rightarrow 4(j+1)} \\
b_{(j+1)_{s}, 5 s}=-f_{2}(s, 1) \kappa^{\ell}\left(\alpha_{3(j+m+4)}\right) w_{4(j+m)+3 \rightarrow 4(j+m+1)+3} \otimes w_{4 j+3 \rightarrow 4(j+1)} \\
b_{2 s+(j+1)_{s}, 5 s}=f_{2}(s, 1) \kappa^{\ell}\left(\alpha_{3(j+m+4)}\right) w_{4(j+m+1+f(s, 1))+1 \rightarrow 4(j+m+1)+3} \otimes w_{4 j+3 \rightarrow 4(j+s+1+f(s, 1))+1} \\
b_{5 s+(j+1)_{s}, 5 s}=-f_{2}(s, 1) \kappa^{\ell}\left(\alpha_{3(j+m+4)}\right) w_{4(j+m+1+f(s, 1))+2 \rightarrow 4(j+m+1)+3} \otimes w_{4 j+3 \rightarrow 4(j+s+1+f(s, 1))+2} .
\end{gathered}
$$

(6) If $r_{0}=5$, then $\Omega^{5}\left(Y_{t}^{(17)}\right)$ is described with $(7 s \times 8 s)$-matrix with the following nonzero elements:

$$
\begin{gathered}
b_{(j+1)_{s}+f(s, 1), 0}=-e_{4(j+m+1)+1} \otimes w_{4 j \rightarrow 4(j+1)} ; \\
b_{s+(j+1)_{s}-f(s, 1), s}=-e_{4(j+m+1)+1} \otimes w_{4 j \rightarrow 4(j+1)} ; \\
b_{(j+1)_{s}, 4 s+f(s, 1)=} f_{2}(s, 1) \kappa^{\ell}\left(\alpha_{3(j+m+4)}\right) w_{4(j+m+1)+1 \rightarrow 4(j+m+1)+3} \otimes w_{4 j+2 \rightarrow 4(j+1)} ; \\
b_{s+(j+1)_{s}, 4 s+f(s, 1)=} f_{2}(s, 1) \kappa^{\ell}\left(\alpha_{3(j+m+4)}\right) w_{4(j+m+s+1)+1 \rightarrow 4(j+m+1)+3} \otimes w_{4 j+2 \rightarrow 4(j+1)} ;
\end{gathered}
$$


HOCHSCHILD COHOMOLOGY RING FOR SELF-INJECTIVE ALGEBRAS OF TREE CLASS $E_{6}$. II. 117

$$
\begin{aligned}
b_{4 s+(j+1)_{s}, 4 s+f(s, 1)} & =-f_{2}(s, 1) \kappa^{\ell}\left(\alpha_{3(j+m+4)}\right) e_{4(j+m+1)+3} \otimes w_{4 j+2 \rightarrow 4(j+1)+2} ; \\
b_{2 s+(j+1)_{s}, 6 s} & =\kappa^{\ell}\left(\alpha_{3(j+m+5)}\right) e_{4(j+m+2)} \otimes w_{4 j+3 \rightarrow 4(j+1+f(s, 1))+1} .
\end{aligned}
$$

(7) If $r_{0}=6$, then $\Omega^{6}\left(Y_{t}^{(17)}\right)$ is described with $(6 s \times 9 s)$-matrix with the following nonzero elements:

$$
\begin{gathered}
b_{(j+1)_{s}, s+f(s, 1)}=w_{4(j+m+1) \rightarrow 4(j+m+s+1)+2} \otimes w_{4(j+s)+1 \rightarrow 4(j+1)} ; \\
b_{(j+1)_{s}, 3 s+f(s, 1)}=w_{4(j+m+1) \rightarrow 4(j+m+1)+1} \otimes w_{4(j+s)+2 \rightarrow 4(j+1)} ;
\end{gathered}
$$

$b_{s+(j+1)_{s}, 5 s}=f_{2}(s, 1) \kappa^{\ell+1}\left(\alpha_{3(j+m-1)}\right) w_{4(j+m+s+1+f(s, 1))+1 \rightarrow 4(j+m+2)} \otimes w_{4 j+3 \rightarrow 4(j+s+1+f(s, 1))+1}$.

(8) If $r_{0}=7$, then $\Omega^{7}\left(Y_{t}^{(17)}\right)$ is described with $(8 s \times 8 s)$-matrix with the following nonzero elements:

$$
\begin{gathered}
b_{(j+1)_{s}+f(s, 1), 0}=-e_{4(j+m+1)+2} \otimes w_{4 j \rightarrow 4(j+1)} ; \\
b_{s+(j+1)_{s}-f(s, 1), s}=e_{4(j+m+1)+2} \otimes w_{4 j \rightarrow 4(j+1)} ; \\
b_{(j+1)_{s}, 4 s+f(s, 1)}=\kappa^{\ell+1}\left(\alpha_{3(j+m-1)}\right) w_{4(j+m+1)+2 \rightarrow 4(j+m+2)} \otimes w_{4 j+2 \rightarrow 4(j+1)} \\
b_{4 s+(j+1)_{s}, 4 s+f(s, 1)}=-\kappa^{\ell+1}\left(\alpha_{3(j+m-1)}\right) e_{4(j+m+2)} \otimes w_{4 j+2 \rightarrow 4(j+1)+2} \\
b_{2 s+(j+1)_{s}, 6 s+f(s, 1)}=w_{4(j+m+1)+3 \rightarrow 4(j+m+2)+1} \otimes w_{4 j+3 \rightarrow 4(j+1)+1} \\
b_{4 s+(j+1)_{s}, 6 s+f(s, 1)}=w_{4(j+m+2) \rightarrow 4(j+m+2)+1} \otimes w_{4 j+3 \rightarrow 4(j+1)+2} \\
b_{5 s+(j+1)_{s}, 6 s+f(s, 1)}=w_{4(j+m+2) \rightarrow 4(j+m+2)+1} \otimes w_{4 j+3 \rightarrow 4(j+s+1)+2} .
\end{gathered}
$$

(9) If $r_{0}=8$ and $s=1$, then $\Omega^{8}\left(Y_{t}^{(17)}\right)$ is described with $(9 s \times 6 s)$-matrix with the following nonzero elements:

$$
\begin{gathered}
b_{j, 0}=-\kappa^{\ell+1}\left(\alpha_{3(j+m-3)}\right) e_{4(j+m)+3} \otimes e_{4 j} ; \\
b_{j-s, s}=\kappa^{\ell+1}\left(\alpha_{3(j+m-3)}\right) w_{4(j+m)+3 \rightarrow 4(j+m)} \otimes e_{4 j} ; \\
b_{j-s, 5 s}=-w_{4(j+m+1)+1 \rightarrow 4(j+m+1)+2} \otimes w_{4(j+1)+2 \rightarrow 4 j+2} ; \\
b_{j-6 s, 6 s}=w_{4(j+m)+3 \rightarrow 4(j+m)+1} \otimes w_{4(j+1)+2 \rightarrow 4 j} ; \\
b_{j-4 s, 7 s}=-w_{4(j+m)+1 \rightarrow 4(j+m)+2} \otimes w_{4 j+2 \rightarrow 4(j+1)+2} ; \\
b_{j-8 s, 8 s}=-\kappa^{\ell+1}\left(\alpha_{3(j+m-3)}\right) w_{4(j+m)+3 \rightarrow 4(j+m+1)+3} \otimes w_{4 j+3 \rightarrow 4 j} ; \\
b_{j-6 s, 8 s}=-\kappa^{\ell+1}\left(\alpha_{3(j+m-3)}\right) w_{4(j+m+1)+2 \rightarrow 4(j+m)+3} \otimes w_{4 j+3 \rightarrow 4(j+1)+1} ; \\
b_{j-5 s, 8 s}=-\kappa^{\ell+1}\left(\alpha_{3(j+m-3)}\right) w_{4(j+m+1)+1 \rightarrow 4(j+m)+3} \otimes w_{4 j+3 \rightarrow 4 j+2} ;
\end{gathered}
$$

(10) If $r_{0}=8$ and $s>1$, then $\Omega^{8}\left(Y_{t}^{(17)}\right)$ is described with $(9 s \times 6 s)$-matrix with the following nonzero elements:

$$
\begin{gathered}
b_{(j+1)_{s}, 0}=\kappa^{\ell+1}\left(\alpha_{3(j+m-3)}\right) e_{4(j+m)+3} \otimes w_{4 j \rightarrow 4(j+1)} ; \\
b_{(j+1)_{s}, 4 s}=w_{4(j+m)+3 \rightarrow 4(j+m+1)+1} \otimes w_{4 j+2 \rightarrow 4(j+1)} ; \\
b_{3 s+(j+1)_{s}, 5 s}=-w_{4(j+m+1)+1 \rightarrow 4(j+m+1)+2} \otimes w_{4(j+s)+2 \rightarrow 4(j+s+1)+2} ; \\
b_{4 s+(j+1)_{s}, 7 s}=-w_{4(j+m+s+1)+1 \rightarrow 4(j+m+s+1)+2} \otimes w_{4 j+2 \rightarrow 4(j+1)+2} ; \\
b_{(j+1)_{s}, 8 s}=\kappa^{\ell+1}\left(\alpha_{3(j+m-2)}\right) w_{4(j+m)+3 \rightarrow 4(j+m+1)+3} \otimes w_{4 j+3 \rightarrow 4(j+1)} ; \\
b_{2 s+(j+1)_{s}, 8 s}=\kappa^{\ell+1}\left(\alpha_{3(j+m-2)}\right) w_{4(j+m+s+1)+2 \rightarrow 4(j+m+1)+3} \otimes w_{4 j+3 \rightarrow 4(j+s+1)+1} ; \\
b_{3 s+(j+1)_{s}, 8 s}=\kappa^{\ell+1}\left(\alpha_{3(j+m-2)}\right) w_{4(j+m+s+1)+1 \rightarrow 4(j+m+1)+3} \otimes w_{4 j+3 \rightarrow 4(j+1)+2} .
\end{gathered}
$$

(11) If $r_{0}=9$ and $s=1$, then $\Omega^{9}\left(Y_{t}^{(17)}\right)$ is described with $(8 s \times 7 s)$-matrix with the following two nonzero elements:

$$
\begin{gathered}
b_{j-4 s, 4 s}=-\kappa^{\ell+1}\left(\alpha_{3(j+m-2)}\right) e_{4(j+m)+3} \otimes w_{4 j+2 \rightarrow 4 j} ; \\
b_{j-6 s, 6 s}=w_{4(j+m)+3 \rightarrow 4(j+m)+2} \otimes w_{4 j+3 \rightarrow 4 j} .
\end{gathered}
$$


(12) If $r_{0}=9$ and $s>1$, then $\Omega^{9}\left(Y_{t}^{(17)}\right)$ is described with $(8 s \times 7 s)$-matrix with the following two nonzero elements:

$$
\begin{gathered}
b_{(j+1)_{s}, 5 s}=-\kappa^{\ell+1}\left(\alpha_{3(j+m-2)}\right) e_{4(j+m+1)+3} \otimes w_{4 j+2 \rightarrow 4(j+1)} ; \\
b_{(j+1)_{s}, 7 s}=-w_{4(j+m+1)+3 \rightarrow 4(j+m+2)+2} \otimes w_{4 j+3 \rightarrow 4(j+1)} .
\end{gathered}
$$

(13) If $r_{0}=10$ and $s=1$, then $\Omega^{10}\left(Y_{t}^{(17)}\right)$ is described with $(9 s \times 6 s)$-matrix with the following nonzero elements:

$$
\begin{gathered}
b_{j, 0}=-\kappa^{\ell+1}\left(\alpha_{3(j+m-2)}\right) e_{4(j+m)} \otimes e_{4 j} ; \\
b_{j+s, 2 s}=w_{4(j+m+1)+2 \rightarrow 4(j+m)+2} \otimes w_{4 j+1 \rightarrow 4 j+2} ; \\
b_{j+3 s, 2 s}=w_{4(j+m)+3 \rightarrow 4(j+m)+2} \otimes w_{4 j+1 \rightarrow 4 j+3} ; \\
b_{j, 4 s}=w_{4(j+m)+2 \rightarrow 4(j+m+1)+2} \otimes w_{4(j+1)+1 \rightarrow 4(j+1)+2} ; \\
b_{j+s, 4 s}=-w_{4(j+m)+3 \rightarrow 4(j+m+1)+2} \otimes w_{4(j+1)+1 \rightarrow 4 j+3} ; \\
b_{j-2 s, 7 s}=-\kappa^{\ell+1}\left(\alpha_{3(j+m-2)}\right) e_{4(j+m)+3} \otimes e_{4 j+3} .
\end{gathered}
$$

(14) If $r_{0}=10$ and $s>1$, then $\Omega^{10}\left(Y_{t}^{(17)}\right)$ is described with $(9 s \times 6 s)$-matrix with the following nonzero elements:

$$
\begin{gathered}
b_{(j+1)_{s}, 0}=\kappa^{\ell+1}\left(\alpha_{3(j+m-2)}\right) e_{4(j+m+1)} \otimes w_{4 j \rightarrow 4(j+1)} ; \\
b_{j+s, 2 s}=w_{4(j+m+s)+2 \rightarrow 4(j+m+s+1)+2} \otimes w_{4 j+1 \rightarrow 4 j+2} ; \\
b_{j+3 s, 2 s}=w_{4(j+m)+3 \rightarrow 4(j+m+s+1)+2} \otimes w_{4 j+1 \rightarrow 4 j+3} ; \\
b_{j, 4 s}=w_{4(j+m)+2 \rightarrow 4(j+m+1)+2} \otimes w_{4(j+s)+1 \rightarrow 4(j+s)+2} ; \\
b_{j+s, 4 s}=-w_{4(j+m)+3 \rightarrow 4(j+m+1)+2} \otimes w_{4(j+s)+1 \rightarrow 4 j+3} ; \\
b_{j-2 s, 7 s}=-\kappa^{\ell+1}\left(\alpha_{3(j+m-2)}\right) w_{4(j+m)+3 \rightarrow 4(j+m+1)+3} \otimes e_{4 j+3} .
\end{gathered}
$$

(II) Represent an arbitrary $t_{0} \in \mathbb{N}$ in the form $t_{0}=11 \ell_{0}+r_{0}$, where $0 \leqslant r_{0} \leqslant 10$. Then $\Omega^{t_{0}}\left(Y_{t}^{(17)}\right)$ is a $\Omega^{r_{0}}\left(Y_{t}^{(17)}\right)$, whose left components twisted by $\sigma^{\ell_{0}}$, and coefficients multiplied by $(-1)^{\ell_{0}}$.

Proposition 34 (Translates for the case 18). (I) Let $r_{0} \in \mathbb{N}, r_{0}<11$. $r_{0}$-translates of the elements $Y_{t}^{(18)}$ are described by the following way.

(1) If $r_{0}=0$, then $\Omega^{0}\left(Y_{t}^{(18)}\right)$ is described with $(6 s \times 6 s)$-matrix with the following elements $b_{i j}$ : If $s \leqslant j<3 s$, then

$$
b_{i j}=\left\{\begin{array}{l}
w_{4(j+m+s)+1 \rightarrow 4(j+m+s)+2} \otimes e_{4(j+s)+1}, \quad i=j \\
0, \quad \text { otherwise. }
\end{array}\right.
$$

If $3 s \leqslant j<5 s$, then $b_{i j}=0$.

If $5 s \leqslant j<6 s$, then

$$
b_{i j}=\left\{\begin{array}{l}
-\kappa^{\ell}\left(\alpha_{3(j+m+4+1)}\right) w_{4(j+m)+3 \rightarrow 4(j+m+1)} \otimes e_{4 j+3}, \quad i=j \\
0, \quad \text { otherwise. }
\end{array}\right.
$$

(2) If $r_{0}=1$, then $\Omega^{1}\left(Y_{t}^{(18)}\right)$ is described with $(7 s \times 7 s)$-matrix with the following elements $b_{i j}$ : 
HOCHSCHILD COHOMOLOGY RING FOR SELF-INJECTIVE ALGEBRAS OF TREE CLASS E . II. 119 If $0 \leqslant j<s$, then

$$
b_{i j}=\left\{\begin{array}{l}
\kappa_{1} w_{4(j+m)+1 \rightarrow 4(j+m)+3} \otimes e_{4 j}, \quad i=j \\
-\kappa_{1} w_{4(j+m+s)+1 \rightarrow 4(j+m)+3} \otimes e_{4 j}, \quad i=j+s \\
0, \quad \text { otherwise }
\end{array}\right.
$$

where $\kappa_{1}=\kappa^{\ell}\left(\alpha_{3(j+4+m)}\right)$.

If $s \leqslant j<3 s$, then

$$
b_{i j}=\left\{\begin{array}{l}
\kappa_{1} w_{4(j+m+s)+2 \rightarrow 4(j+m+1)} \otimes e_{4(j+s)+1}, \quad i=j+s \\
\kappa_{1} w_{4(j+m)+3 \rightarrow 4(j+m+1)} \otimes w_{4(j+s)+1 \rightarrow 4(j+s)+2}, \quad i=j+3 s \\
0, \quad \text { otherwise }
\end{array}\right.
$$

where $\kappa_{1}=\kappa^{\ell}\left(\alpha_{3(j+m+4+1)}\right)$.

If $3 s \leqslant j<5 s$, then

$$
b_{i j}=\left\{\begin{array}{l}
-w_{4(j+m)+3 \rightarrow 4(j+m+1)+1} \otimes e_{4(j+s)+2}, \quad i=j+s \\
0, \quad \text { otherwise. }
\end{array}\right.
$$

If $5 s \leqslant j<7 s$, then

$$
b_{i j}=\left\{\begin{array}{l}
-w_{4(j+m+s+1)+1 \rightarrow 4(j+m+s+1)+2} \otimes w_{4 j+3 \rightarrow 4(j+1)}, \quad i=(j+s+1)_{2 s} \\
-w_{4(j+m+1) \rightarrow 4(j+m+s+1)+2} \otimes e_{4 j+3}, \quad i=6 s+(j)_{s} \\
0, \quad \text { otherwise. }
\end{array}\right.
$$

(3) If $r_{0}=2$, then $\Omega^{2}\left(Y_{t}^{(18)}\right)$ is described with $(6 s \times 6 s)$-matrix with the following elements $b_{i j}$ : If $0 \leqslant j<s$, then

$$
b_{i j}=\left\{\begin{array}{l}
\kappa^{\ell}\left(\alpha_{3(j+m+4)}\right) w_{4(j+m)-1 \rightarrow 4(j+m)} \otimes e_{4 j}, \quad i=j ; \\
0, \quad \text { otherwise. }
\end{array}\right.
$$

If $s \leqslant j<3 s$, then $b_{i j}=0$.

If $3 s \leqslant j<5 s$, then

$$
b_{i j}=\left\{\begin{array}{l}
-w_{4(j+m)+1 \rightarrow 4(j+m)+2} \otimes e_{4(j+s)+2}, \quad i=j \\
0, \quad \text { otherwise. }
\end{array}\right.
$$

If $5 s \leqslant j<6 s$, then $b_{i j}=0$.

(4) If $r_{0}=3$, then $\Omega^{3}\left(Y_{t}^{(18)}\right)$ is described with $(6 s \times 8 s)$-matrix with the following elements $b_{i j}$ : If $0 \leqslant j<s$, then

$$
b_{i j}=\left\{\begin{array}{l}
\kappa_{1} w_{4(j+m)+2 \rightarrow 4(j+m+1)} \otimes e_{4 j}, \quad i=j \\
\kappa_{1} w_{4(j+m)+3 \rightarrow 4(j+m+1)} \otimes w_{4 j \rightarrow 4 j+1}, \quad i=j+2 s \\
0, \quad \text { otherwise }
\end{array}\right.
$$

where $\kappa_{1}=\kappa^{\ell}\left(\alpha_{3(j+m+4+1)}\right)$. 
If $s \leqslant j<3 s$, then

$$
b_{i j}=\left\{\begin{array}{l}
-w_{4(j+m)+3 \rightarrow 4(j+m+1)+1} \otimes e_{4(j+s)+1}, \quad i=j+s \\
0, \quad \text { otherwise. }
\end{array}\right.
$$

If $3 s \leqslant j<4 s$, then

$$
b_{i j}=\left\{\begin{array}{l}
-f_{1}(j, 4 s-1) e_{4(j+m+1)+2} \otimes w_{4(j+s)+2 \rightarrow 4(j+1)}, \quad i=(j+1)_{2 s} \\
0, \quad \text { otherwise. }
\end{array}\right.
$$

If $4 s \leqslant j<5 s$, then

$$
b_{i j}=\left\{\begin{array}{l}
f_{1}(j, 5 s-1) e_{4(j+m+1)+2} \otimes w_{4(j+s)+2 \rightarrow 4(j+1)}, \quad i=(j+1)_{2 s} \\
0, \quad \text { otherwise. }
\end{array}\right.
$$

If $5 s \leqslant j<6 s$, then

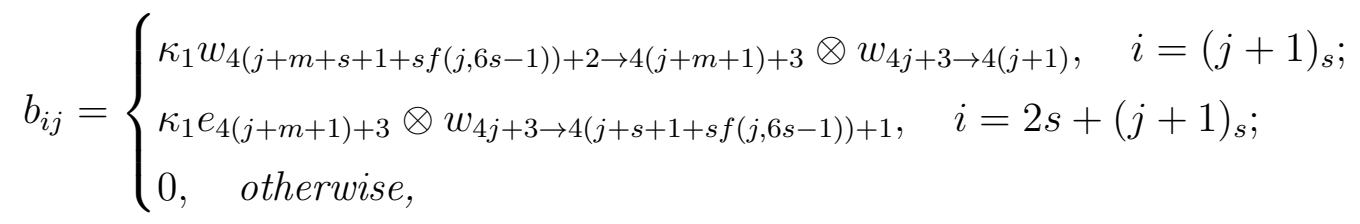

where $\kappa_{1}=-\kappa^{\ell}\left(\alpha_{3(j+m+4+1)}\right) f_{1}(j, 6 s-1)$.

(5) If $r_{0}=4$, then $\Omega^{4}\left(Y_{t}^{(18)}\right)$ is described with $(7 s \times 9 s)$-matrix with the following elements $b_{i j}$ : If $s \leqslant j<2 s$, then

$$
b_{i j}=\left\{\begin{array}{l}
w_{4(j+m)-1 \rightarrow 4(j+m+s)+1} \otimes e_{4 j}, \quad i=j-s \\
0, \quad \text { otherwise. }
\end{array}\right.
$$

If $2 s \leqslant j<3 s$, then

$$
b_{i j}=\left\{\begin{array}{l}
w_{4(j+m+s)+1 \rightarrow 4(j+m+s)+2} \otimes e_{4 j+1}, \quad i=j \\
-e_{4(j+m+s)+2} \otimes w_{4 j+1 \rightarrow 4 j+2}, \quad i=j+3 s \\
0, \quad \text { otherwise. }
\end{array}\right.
$$

If $3 s \leqslant j<4 s$, then

$$
b_{i j}=\left\{\begin{array}{l}
w_{4(j+m+s)+1 \rightarrow 4(j+m+s)+2} \otimes e_{4 j+1}, \quad i=j \\
-e_{4(j+m+s)+2} \otimes w_{4 j+1 \rightarrow 4 j+2}, \quad i=j+4 s \\
0, \quad \text { otherwise. }
\end{array}\right.
$$

If $4 s \leqslant j<6 s$, then

$$
b_{i j}=\left\{\begin{array}{c}
\kappa^{\ell}\left(\alpha_{3(j+4+m)}\right) f_{1}(j, 6 s-1) e_{4(j+m)+3} \otimes w_{4 j+2 \rightarrow 4(j+1)}, \\
i=(j+1)_{s}, j<5 s-1 \text { or } j=6 s-1 \\
0, \quad \text { otherwise. }
\end{array}\right.
$$

If $6 s \leqslant j<7 s$, then $b_{i j}=0$. 
HOCHSCHILD COHOMOLOGY RING FOR SELF-INJECTIVE ALGEBRAS OF TREE CLASS $E_{6}$. II. 121

(6) If $r_{0}=5$, then $\Omega^{5}\left(Y_{t}^{(18)}\right)$ is described with $(6 s \times 8 s)$-matrix with the following elements $b_{i j}$ : If $0 \leqslant j<s$, then

$$
b_{i j}=\left\{\begin{array}{l}
\kappa_{1} w_{4(j+m)+1 \rightarrow 4(j+m)+3} \otimes e_{4 j}, \quad i=j \\
\kappa_{1} w_{4(j+m+s)+1 \rightarrow 4(j+m)+3} \otimes e_{4 j}, \quad i=j+s \\
-\kappa_{1} e_{4(j+m)+3} \otimes w_{4 j \rightarrow 4 j+2}, \quad i=j+4 s \\
-\kappa_{1} e_{4(j+m)+3} \otimes w_{4 j \rightarrow 4(j+s)+2}, \quad i=j+5 s \\
0, \quad \text { otherwise, }
\end{array}\right.
$$

where $\kappa_{1}=-\kappa^{\ell}\left(\alpha_{3(j+4+m)}\right)$.

If $s \leqslant j<3 s$, then

$$
b_{i j}=\left\{\begin{array}{l}
-w_{4(j+m+1)+1 \rightarrow 4(j+m+1)+2} \otimes w_{4(j+s)+1 \rightarrow 4(j+1)}, \quad i=(j+1)_{2 s} ; \\
-w_{4(j+m+1) \rightarrow 4(j+m+1)+2} \otimes e_{4(j+s)+1}, \quad i=j+s \\
0, \quad \text { otherwise. }
\end{array}\right.
$$

If $3 s \leqslant j<5 s$, then $b_{i j}=0$.

If $5 s \leqslant j<6 s$, then

$$
b_{i j}=\left\{\begin{array}{l}
-\kappa^{\ell+1}\left(\alpha_{3(j+m)}\right) f_{1}(j, 6 s-1) e_{4(j+m+2)} \otimes w_{4 j+3 \rightarrow 4(j+s+1+s f(j, 6 s-1))+1}, \quad i=2 s+(j+1)_{s} ; \\
0, \quad \text { otherwise. }
\end{array}\right.
$$

(7) If $r_{0}=6$, then $\Omega^{6}\left(Y_{t}^{(18)}\right)$ is described with $(8 s \times 9 s)$-matrix with the following elements $b_{i j}$ : If $0 \leqslant j<s$, then

$$
b_{i j}=\left\{\begin{array}{l}
w_{4(j+m) \rightarrow 4(j+m+s)+2} \otimes e_{4 j}, \quad i=j \\
-w_{4(j+m+s)+1 \rightarrow 4(j+m+s)+2} \otimes w_{4 j \rightarrow 4(j+s)+1}, \quad i=j+3 s \\
0, \quad \text { otherwise. }
\end{array}\right.
$$

If $s \leqslant j<2 s$, then

$$
b_{i j}=\left\{\begin{array}{l}
-w_{4(j+m) \rightarrow 4(j+m+s)+2} \otimes e_{4 j}, \quad i=j-s ; \\
w_{4(j+m+s)+1 \rightarrow 4(j+m+s)+2} \otimes w_{4 j \rightarrow 4(j+s)+1}, \quad i=j ; \\
0, \quad \text { otherwise. }
\end{array}\right.
$$

If $2 s \leqslant j<3 s$, then

$$
b_{i j}=\left\{\begin{array}{l}
\kappa^{\ell}\left(\alpha_{3(j+m+4)}\right) f_{2}\left((j)_{s}, s-1\right) w_{4(j+m)+1 \rightarrow 4(j+m)+3} \otimes e_{4 j+1}, \quad i=j-s \\
0, \quad \text { otherwise. }
\end{array}\right.
$$

If $3 s \leqslant j<4 s$, then

$$
b_{i j}=\left\{\begin{array}{l}
-\kappa^{\ell}\left(\alpha_{3(j+m+4)}\right) f_{2}\left((j)_{s}, s-1\right) w_{4(j+m)+1 \rightarrow 4(j+m)+3} \otimes e_{4 j+1}, \quad i=j ; \\
0, \quad \text { otherwise. }
\end{array}\right.
$$


If $4 s \leqslant j<6 s$, then

$b_{i j}=\left\{\begin{array}{l}-\kappa^{\ell+1}\left(\alpha_{3(j+m-1)}\right) e_{4(j+m+1)} \otimes w_{4 j+2 \rightarrow 4(j+1)}, \quad i=(j+1)_{s}, j<5 s-1 \text { or } j=6 s-1 ; \\ 0, \quad \text { otherwise. }\end{array}\right.$

If $6 s \leqslant j<7 s-1$, then

$$
b_{i j}=\left\{\begin{array}{l}
w_{4(j+m+1) \rightarrow 4(j+m+s+1)+1} \otimes w_{4 j+3 \rightarrow 4(j+1)}, \quad i=(j+1)_{s} \\
0, \quad \text { otherwise. }
\end{array}\right.
$$

If $7 s-1 \leqslant j<6 s$, then $b_{i j}=0$.

If $6 s \leqslant j<7 s$, then

$$
b_{i j}=\left\{\begin{array}{l}
-e_{4(j+m+s+1)+1} \otimes w_{4 j+3 \rightarrow 4(j+s+1)+1}, \quad i=3 s+(j+1)_{s}-2 s f(j, 7 s-1) ; \\
0, \quad \text { otherwise. }
\end{array}\right.
$$

If $7 s \leqslant j<8 s-1$, then $b_{i j}=0$.

If $8 s-1 \leqslant j<8 s$, then

$$
b_{i j}=\left\{\begin{array}{l}
w_{4(j+m+1) \rightarrow 4(j+m+s+1)+1} \otimes w_{4 j+3 \rightarrow 4(j+1)}, \quad i=(j+1)_{s} \\
0, \quad \text { otherwise. }
\end{array}\right.
$$

If $8 s \leqslant j<7 s$, then $b_{i j}=0$.

If $7 s \leqslant j<8 s$, then

$$
b_{i j}=\left\{\begin{array}{l}
-e_{4(j+m+s+1)+1} \otimes w_{4 j+3 \rightarrow 4(j+s+1)+1}, \quad i=s+(j+1)_{s}+2 s f(j, 8 s-1) \\
0, \quad \text { otherwise. }
\end{array}\right.
$$

(8) If $r_{0}=7$, then $\Omega^{7}\left(Y_{t}^{(18)}\right)$ is described with $(9 s \times 8 s)$-matrix with the following elements $b_{i j}$ : If $s \leqslant j<2 s$, then

$$
b_{i j}=\left\{\begin{array}{l}
-\kappa_{1} w_{4(j+m)+2 \rightarrow 4(j+m+1)} \otimes e_{4 j}, \quad i=j \\
\kappa_{1} w_{4(j+m)+3 \rightarrow 4(j+m+1)} \otimes w_{4 j \rightarrow 4(j+s)+1}, \quad i=j+s \\
\kappa_{1} e_{4(j+m+1)} \otimes w_{4 j \rightarrow 4(j+s)+2}, \quad i=j+3 s \\
\kappa_{1} e_{4(j+m+1)} \otimes w_{4 j \rightarrow 4 j+2}, \quad i=j+4 s \\
0, \quad \text { otherwise, }
\end{array}\right.
$$

where $\kappa_{1}=\kappa^{\ell+1}\left(\alpha_{3(j+m-1)}\right)$.

If $2 s \leqslant j<4 s$, then

$$
b_{i j}=\left\{\begin{array}{l}
w_{4(j+m)+3 \rightarrow 4(j+m+1)+1} \otimes e_{4 j+1}, \quad i=j \\
w_{4(j+m+1) \rightarrow 4(j+m+1)+1} \otimes w_{4 j+1 \rightarrow 4 j+2}, \quad i=j+2 s \\
-e_{4(j+m+1)+1} \otimes w_{4 j+1 \rightarrow 4 j+3}, \quad i=j+4 s \\
0, \quad \text { otherwise. }
\end{array}\right.
$$

If $4 s \leqslant j<9 s$, then $b_{i j}=0$. 
HOCHSCHILD COHOMOLOGY RING FOR SELF-INJECTIVE ALGEBRAS OF TREE CLASS $E_{6}$. II. 123

(9) If $r_{0}=8$, then $\Omega^{8}\left(Y_{t}^{(18)}\right)$ is described with $(8 s \times 6 s)$-matrix with the following elements $b_{i j}$ : If $0 \leqslant j<s$, then

$$
b_{i j}=\left\{\begin{array}{l}
w_{4(j+m)-1 \rightarrow 4(j+m+s)+1} \otimes e_{4 j}, \quad i=j \\
e_{4(j+m+s)+1} \otimes w_{4 j \rightarrow 4 j+2}, \quad i=j+3 s \\
0, \quad \text { otherwise. }
\end{array}\right.
$$

If $s \leqslant j<2 s$, then

$$
b_{i j}=\left\{\begin{array}{l}
e_{4(j+m+s)+1} \otimes w_{4 j \rightarrow 4 j+2}, \quad i=j+3 s \\
0, \quad \text { otherwise. }
\end{array}\right.
$$

If $2 s \leqslant j<4 s$, then

$$
b_{i j}=\left\{\begin{array}{c}
\kappa^{\ell+1}\left(\alpha_{3(j+m-1)}\right) w_{4(j+m)+3 \rightarrow 4(j+m+1)} \otimes w_{4 j+1 \rightarrow 4(j+1)} \\
i=(j+1)_{s}, j<3 s-1 \text { or } j=4 s-1 ; \\
\kappa^{\ell+1}\left(\alpha_{3(j+m-1)}\right) w_{4(j+m)+2 \rightarrow 4(j+m+1)} \otimes e_{4 j+1}, \quad i=j-s \\
0, \quad \text { otherwise. }
\end{array}\right.
$$

If $4 s \leqslant j<6 s$, then $b_{i j}=0$.

If $6 s \leqslant j<8 s$, then

$$
b_{i j}=\left\{\begin{array}{l}
-f_{1}(j, 7 s) e_{4(j+m+s+1)+2} \otimes w_{4 j+3 \rightarrow 4(j+s+1)+1}, \quad i=s+(j+s+1)_{2 s} ; \\
0, \quad \text { otherwise. }
\end{array}\right.
$$

(10) If $r_{0}=9$, then $\Omega^{9}\left(Y_{t}^{(18)}\right)$ is described with $(9 s \times 7 s)$-matrix with the following elements $b_{i j}$ :

If $0 \leqslant j<s$, then

$$
b_{i j}=\left\{\begin{array}{l}
-\kappa^{\ell+1}\left(\alpha_{3(j+m-1)}\right) w_{4(j+m)+3 \rightarrow 4(j+m+1)} \otimes e_{4 j}, \quad i=j ; \\
0, \quad \text { otherwise. }
\end{array}\right.
$$

If $s \leqslant j<2 s$, then $b_{i j}=0$.

If $2 s \leqslant j<3 s$, then

$$
b_{i j}=\left\{\begin{array}{l}
w_{4(j+m+1) \rightarrow 4(j+m+1)+2} \otimes e_{4 j+1}, \quad i=j-s \\
-e_{4(j+m+1)+2} \otimes w_{4 j+1 \rightarrow 4 j+3}, \quad i=j+3 s \\
0, \quad \text { otherwise. }
\end{array}\right.
$$

If $3 s \leqslant j<4 s$, then $b_{i j}=0$.

If $4 s \leqslant j<5 s$, then

$$
b_{i j}=\left\{\begin{array}{l}
w_{4(j+m+1) \rightarrow 4(j+m+s+1)+2} \otimes e_{4(j+s)+1}, \quad i=j-2 s \\
-e_{4(j+m+s+1)+2} \otimes w_{4(j+s)+1 \rightarrow 4 j+3}, \quad i=j+2 s \\
0, \quad \text { otherwise. }
\end{array}\right.
$$

If $5 s \leqslant j<7 s$, then $b_{i j}=0$. 
If $7 s \leqslant j<8 s$, then

$$
b_{i j}=\left\{\begin{array}{l}
-\kappa^{\ell+1}\left(\alpha_{3(j+m-1)}\right) f_{1}(j, 8 s-1) e_{4(j+m+1)+3} \otimes w_{4 j+3 \rightarrow 4(j+1)}, \quad i=(j+1)_{s} \\
0, \quad \text { otherwise. }
\end{array}\right.
$$

If $8 s \leqslant j<9 s$, then $b_{i j}=0$.

(11) If $r_{0}=10$, then $\Omega^{10}\left(Y_{t}^{(18)}\right)$ is described with $(8 s \times 6 s)$-matrix with the following elements $b_{i j}$ :

If $0 \leqslant j<s$, then

$$
b_{i j}=\left\{\begin{array}{l}
w_{4(j+m) \rightarrow 4(j+m+s)+2} \otimes e_{4 j}, \quad i=j ; \\
-w_{4(j+m+s)+1 \rightarrow 4(j+m+s)+2} \otimes w_{4 j \rightarrow 4 j+1}, \quad i=j+s ; \\
e_{4(j+m+s)+2} \otimes w_{4 j \rightarrow 4 j+2}, \quad i=j+3 s \\
0, \quad \text { otherwise. }
\end{array}\right.
$$

If $s \leqslant j<2 s$, then

$$
b_{i j}=\left\{\begin{array}{l}
-w_{4(j+m) \rightarrow 4(j+m+s)+2} \otimes e_{4 j}, \quad i=j-s ; \\
-w_{4(j+m+s)+1 \rightarrow 4(j+m+s)+2} \otimes w_{4 j \rightarrow 4 j+1}, \quad i=j+s ; \\
e_{4(j+m+s)+2} \otimes w_{4 j \rightarrow 4 j+2}, \quad i=j+3 s \\
0, \quad \text { otherwise. }
\end{array}\right.
$$

If $2 s \leqslant j<3 s$, then

$$
b_{i j}=\left\{\begin{array}{l}
-\kappa_{1} w_{4(j+m+s)+1 \rightarrow 4(j+m)+3} \otimes e_{4 j+1}, \quad i=j-s \\
\kappa_{1} w_{4(j+m+s)+2 \rightarrow 4(j+m)+3} \otimes w_{4 j+1 \rightarrow 4 j+2}, \quad i=j+s \\
\kappa_{1} e_{4(j+m)+3} \otimes w_{4 j+1 \rightarrow 4 j+3}, \quad i=j+3 s \\
0, \quad \text { otherwise, }
\end{array}\right.
$$

where $\kappa_{1}=\kappa^{\ell+1}\left(\alpha_{3(j+m-2)}\right)$.

If $3 s \leqslant j<4 s$, then

$$
b_{i j}=\left\{\begin{array}{l}
-\kappa_{1} w_{4(j+m+s)+1 \rightarrow 4(j+m)+3} \otimes e_{4 j+1}, \quad i=j-s ; \\
\kappa_{1} w_{4(j+m+s)+2 \rightarrow 4(j+m)+3} \otimes w_{4 j+1 \rightarrow 4 j+2}, \quad i=j+s ; \\
-\kappa_{1} e_{4(j+m)+3} \otimes w_{4 j+1 \rightarrow 4 j+3}, \quad i=j+2 s \\
0, \quad \text { otherwise, }
\end{array}\right.
$$

where $\kappa_{1}=\kappa^{\ell+1}\left(\alpha_{3(j+m-2)}\right)$.

If $4 s \leqslant j<8 s$, then $b_{i j}=0$.

(II) Represent an arbitrary $t_{0} \in \mathbb{N}$ in the form $t_{0}=11 \ell_{0}+r_{0}$, where $0 \leqslant r_{0} \leqslant 10$. Then $\Omega^{t_{0}}\left(Y_{t}^{(18)}\right)$ is a $\Omega^{r_{0}}\left(Y_{t}^{(18)}\right)$, whose left components twisted by $\sigma^{\ell_{0}}$.

Proposition 35 (Translates for the case 19). (I) Let $r_{0} \in \mathbb{N}, r_{0}<11$. $r_{0}$-translates of the elements $Y_{t}^{(19)}$ are described by the following way. 
HOCHSCHILD COHOMOLOGY RING FOR SELF-INJECTIVE ALGEBRAS OF TREE CLASS $E_{6}$. II. 125

(1) If $r_{0}=0$, then $\Omega^{0}\left(Y_{t}^{(19)}\right)$ is described with $(7 s \times 6 s)$-matrix with the following two nonzero elements:

$$
\begin{gathered}
b_{s, s}=\kappa^{\ell}\left(\alpha_{3(j+5)}\right) w_{4(j+m+s)+1 \rightarrow 4(j+m+1)} \otimes e_{4(j+s)+1} ; \\
b_{2 s, 2 s}=\kappa^{\ell}\left(\alpha_{3(j+5)}\right) w_{4(j+m+s)+1 \rightarrow 4(j+m+1)} \otimes e_{4(j+s)+1} .
\end{gathered}
$$

(2) If $r_{0}=1$, then $\Omega^{1}\left(Y_{t}^{(19)}\right)$ is described with $(6 s \times 7 s)$-matrix with the following two nonzero elements:

$$
\begin{gathered}
b_{j,(3)_{s}}=-\kappa^{\ell}\left(\alpha_{3(j+m+5)}\right) w_{4(j+m)+1 \rightarrow 4(j+m+1)} \otimes e_{4 j} ; \\
b_{j+s,(3)_{s}}=\kappa^{\ell}\left(\alpha_{3(j+m+5)}\right) w_{4(j+m+s)+1 \rightarrow 4(j+m+1)} \otimes e_{4 j} .
\end{gathered}
$$

(3) If $r_{0}=2$, then $\Omega^{2}\left(Y_{t}^{(19)}\right)$ is described with $(6 s \times 6 s)$-matrix with the following nonzero elements:

$$
\begin{gathered}
b_{(j+1)_{s},(2)_{s}}=-f_{2}(s, 1) \kappa^{\ell}\left(\alpha_{3(j+m+5)}\right) w_{4(j+m)+3 \rightarrow 4(j+m+1)} \otimes w_{4 j \rightarrow 4(j+1)} ; \\
b_{(j+1)_{s}, s+(2)_{s}}=f_{2}(s, 1) w_{4(j+m)+3 \rightarrow 4(j+m+1)+1} \otimes w_{4(j+s)+1 \rightarrow 4(j+1)} ; \\
b_{(j+1)_{s}, 2 s+(2)_{s}}=-f_{2}(s, 1) w_{4(j+m)+3 \rightarrow 4(j+m+1)+1} \otimes w_{4(j+s)+1 \rightarrow 4(j+1)} .
\end{gathered}
$$

(4) If $r_{0}=3$, then $\Omega^{3}\left(Y_{t}^{(19)}\right)$ is described with $(7 s \times 8 s)$-matrix with the following two nonzero elements:

$$
\begin{aligned}
b_{s+(j+1)_{s}-f(s, 1), 2 s+(2)_{s}} & =-f_{2}(s, 1) e_{4(j+m+s+1)+2} \otimes w_{4 j+1 \rightarrow 4(j+1)} ; \\
b_{(j+1)_{s}+f(s, 1), 3 s+(2)_{s}} & =f_{2}(s, 1) e_{4(j+m+s+1)+2} \otimes w_{4 j+1 \rightarrow 4(j+1)} .
\end{aligned}
$$

(5) If $r_{0}=4$, then $\Omega^{4}\left(Y_{t}^{(19)}\right)$ is described with $(6 s \times 9 s)$-matrix with the following nonzero elements:

$$
\begin{gathered}
b_{(j+1)_{s},(2)_{s}}=-\kappa^{\ell}\left(\alpha_{3(j+m+4)}\right) e_{4(j+m)+3} \otimes w_{4 j \rightarrow 4(j+1)} ; \\
b_{(j+1)_{s}, s+(2)_{s}+f(s, 1)}=w_{4(j+m)+3 \rightarrow 4(j+m+1)+2} \otimes w_{4(j+s)+1 \rightarrow 4(j+1)} ; \\
b_{s+(j+1)_{s}, s+(2)_{s}+f(s, 1)}=-w_{4(j+m+1) \rightarrow 4(j+m+1)+2} \otimes w_{4(j+s)+1 \rightarrow 4(j+1)} ; \\
b_{s+(j+1)_{s}, 2 s+(2)_{s}-f(s, 1)}=w_{4(j+m+1) \rightarrow 4(j+m+1)+2} \otimes w_{4(j+s)+1 \rightarrow 4(j+1)} .
\end{gathered}
$$

(6) If $r_{0}=5$, then $\Omega^{5}\left(Y_{t}^{(19)}\right)$ is described with $(8 s \times 8 s)$-matrix with the following nonzero elements:

$$
\begin{gathered}
b_{s+(j+1)_{s}-f(s, 1),(2)_{s}}=w_{4(j+m+s+1)+1 \rightarrow 4(j+m+s+1)+2} \otimes w_{4 j \rightarrow 4(j+1)} ; \\
b_{(j+1)_{s}+f(s, 1), s+(2)_{s}}=-w_{4(j+m+s+1)+1 \rightarrow 4(j+m+s+1)+2} \otimes w_{4 j \rightarrow 4(j+1)} ; \\
b_{(j+1)_{s}+f(s, 1), 2 s+(2)_{s}}=-\kappa^{\ell+1}\left(\alpha_{3(j+m-1)}\right) w_{4(j+m+1)+1 \rightarrow 4(j+m+1)+3} \otimes w_{4 j+1 \rightarrow 4(j+1)} ; \\
b_{s+(j+1)_{s}-f(s, 1), 3 s+(2)_{s}}=-\kappa^{\ell+1}\left(\alpha_{3(j+m-1)}\right) w_{4(j+m+1)+1 \rightarrow 4(j+m+1)+3} \otimes w_{4 j+1 \rightarrow 4(j+1)} .
\end{gathered}
$$

(7) If $r_{0}=6$, then $\Omega^{6}\left(Y_{t}^{(19)}\right)$ is described with $(9 s \times 9 s)$-matrix with the following nonzero elements:

$$
\begin{gathered}
b_{(j+1)_{s}, s+(2)_{s}}=-\kappa^{\ell+1}\left(\alpha_{3(j+m-1)}\right) e_{4(j+m+1)} \otimes w_{4 j \rightarrow 4(j+1)} ; \\
b_{j-s, 2 s+(2)_{s}}=w_{4(j+m)+1 \rightarrow 4(j+m+1)+1} \otimes e_{4 j+1} ; \\
b_{j, 3 s+(2)_{s}}=w_{4(j+m)+1 \rightarrow 4(j+m+1)+1} \otimes e_{4 j+1} .
\end{gathered}
$$

(8) If $r_{0}=7$, then $\Omega^{7}\left(Y_{t}^{(19)}\right)$ is described with $(8 s \times 8 s)$-matrix with the following nonzero elements:

$$
\begin{gathered}
b_{j+s,(2)_{s}}=w_{4(j+m+s)+2 \rightarrow 4(j+m+s+1)+1} \otimes e_{4 j} ; \\
b_{j+2 s,(2)_{s}}=-w_{4(j+m)+3 \rightarrow 4(j+m+s+1)+1} \otimes w_{4 j \rightarrow 4 j+1} ; \\
b_{j+4 s,(2)_{s}}=-w_{4(j+m+1) \rightarrow 4(j+m+s+1)+1} \otimes w_{4 j \rightarrow 4 j+2} ; \\
b_{j+5 s,(2)_{s}}=-w_{4(j+m+1) \rightarrow 4(j+m+s+1)+1} \otimes w_{4 j \rightarrow 4(j+s)+2} ;
\end{gathered}
$$




$$
\begin{gathered}
b_{j+s, s+(2)_{s}}=-w_{4(j+m)+3 \rightarrow 4(j+m+s+1)+1} \otimes w_{4 j \rightarrow 4(j+s)+1} ; \\
b_{j+3 s, s+(2)_{s}}=-w_{4(j+m+1) \rightarrow 4(j+m+s+1)+1} \otimes w_{4 j \rightarrow 4(j+s)+2} ; \\
b_{j+4 s, s+(2)_{s}}=-w_{4(j+m+1) \rightarrow 4(j+m+s+1)+1} \otimes w_{4 j \rightarrow 4 j+2} .
\end{gathered}
$$

(9) If $r_{0}=8$ and $s=2$, then $\Omega^{8}\left(Y_{t}^{(19)}\right)$ is described with $(9 s \times 6 s)$-matrix with the following nonzero elements:

$$
\begin{gathered}
b_{j-s, 2 s+1}=w_{4(j+m)+2 \rightarrow 4(j+m+1)+2} \otimes e_{4 j+1} ; \\
b_{(j+1)_{s}, 3 s+1}=w_{4(j+m)+3 \rightarrow 4(j+m+s+1)+1} \otimes w_{4 j+1 \rightarrow 4(j+1)} \\
b_{s+(j+1)_{s}, 4 s+1}=e_{4(j+m+s+1)+2} \otimes w_{4(j+2)+1 \rightarrow 4(j+s+1)+1} .
\end{gathered}
$$

(10) If $r_{0}=8$ and $s \neq 2$, then $\Omega^{8}\left(Y_{t}^{(19)}\right)$ is described with $(9 s \times 6 s)$-matrix with the following nonzero elements:

$$
\begin{gathered}
b_{(j+1)_{s}, s+1+f(s, 1)}=w_{4(j+m)+3 \rightarrow 4(j+m+1+f(s, 1))+1} \otimes w_{4(j+s+f(s, 1))+1 \rightarrow 4(j+1)} ; \\
b_{s+(j+1)_{s}, 2 s+1+f(s, 1)}=e_{4(j+m+1+f(s, 1))+2} \otimes w_{4(j+f(s, 1))+1 \rightarrow 4(j+1+f(s, 1))+1} ; \\
b_{j-2 s+f(s, 1), 4 s+1-3 f(s, 1)}=w_{4(j+m+s+f(s, 1))+2 \rightarrow 4(j+m+s+1+f(s, 1))+2} \otimes e_{4(j+s+f(s, 1))+1} .
\end{gathered}
$$

(11) If $r_{0}=9$ and $s=2$, then $\Omega^{9}\left(Y_{t}^{(19)}\right)$ is described with $(8 s \times 7 s)$-matrix with the following nonzero elements:

$$
\begin{gathered}
b_{j, s+1}=-w_{4(j+m+1) \rightarrow 4(j+m+s+1)+2} \otimes w_{4 j \rightarrow 4(j+2)+1} \\
b_{j+s, s+1}=w_{4(j+m+1) \rightarrow 4(j+m+s+1)+2} \otimes w_{4 j \rightarrow 4 j+1} \\
b_{(j+1)_{s}, 3 s+1}=e_{4(j+m+1)+3} \otimes w_{4 j+1 \rightarrow 4(j+1)} .
\end{gathered}
$$

(12) If $r_{0}=9$ and $s \neq 2$, then $\Omega^{9}\left(Y_{t}^{(19)}\right)$ is described with $(8 s \times 7 s)$-matrix with the following nonzero elements:

$$
\begin{gathered}
b_{j-f(s, 1), 1}=f_{2}(s, 1) w_{4(j+m)+3 \rightarrow 4(j+m+s+1)+2} \otimes e_{4 j} ; \\
b_{j-s, 2 s+1}=\kappa^{\ell+1}\left(\alpha_{3(j+m-1)}\right) w_{4(j+m+1) \rightarrow 4(j+m+1)+3} \otimes e_{4 j+1} ; \\
b_{j+3 s, 2 s+1}=-\kappa^{\ell+1}\left(\alpha_{3(j+m-1)}\right) w_{4(j+m+1)+2 \rightarrow 4(j+m+1)+3} \otimes w_{4 j+1 \rightarrow 4 j+3} .
\end{gathered}
$$

(13) If $r_{0}=10$ and $s=1$, then $\Omega^{10}\left(Y_{t}^{(19)}\right)$ is described with $(6 s \times 6 s)$-matrix with the following nonzero elements:

$$
\begin{gathered}
b_{j, 0}=\kappa^{\ell+1}\left(\alpha_{3(j+m-1)}\right) w_{4(j+m) \rightarrow 4(j+m)+3} \otimes e_{4 j} ; \\
b_{j+s, 0}=-\kappa^{\ell+1}\left(\alpha_{3(j+m-1)}\right) w_{4(j+m+1)+1 \rightarrow 4(j+m)+3} \otimes w_{4 j \rightarrow 4 j+1} ; \\
b_{j+3 s, 0}=\kappa^{\ell+1}\left(\alpha_{3(j+m-1)}\right) w_{4(j+m+1)+2 \rightarrow 4(j+m)+3} \otimes w_{4 j \rightarrow 4 j+2} ; \\
b_{j-s, s}=-w_{4(j+m) \rightarrow 4(j+m+1)+2} \otimes w_{4(j+1)+1 \rightarrow 4 j} ; \\
b_{j+s, s}=-w_{4(j+m+1)+1 \rightarrow 4(j+m+1)+2} \otimes w_{4(j+1)+1 \rightarrow 4 j+1} .
\end{gathered}
$$

(14) If $r_{0}=10$ and $s=2$, then $\Omega^{10}\left(Y_{t}^{(19)}\right)$ is described with $(6 s \times 6 s)$-matrix with the following nonzero elements:

$$
\begin{gathered}
b_{j+s, 1}=-w_{4(j+m+2)+1 \rightarrow 4(j+m)+3} \otimes w_{4 j \rightarrow 4 j+1} ; \\
b_{j+2 s, 1}=w_{4(j+m)+1 \rightarrow 4(j+m)+3} \otimes w_{4 j \rightarrow 4(j+2)+1} ; \\
b_{j+3 s, 1}=w_{4(j+m+s)+2 \rightarrow 4(j+m)+3} \otimes w_{4 j \rightarrow 4 j+2} ; \\
b_{j+4 s, 1}=-w_{4(j+m)+2 \rightarrow 4(j+m)+3} \otimes w_{4 j \rightarrow 4(j+s)+2} ; \\
b_{j+5 s, 1}=e_{4(j+m)+3} \otimes w_{4 j \rightarrow 4 j+3} ; \\
b_{2 s+(j+1)_{s}, 2 s}=-w_{4(j+m+1)+1 \rightarrow 4(j+m+1)+2} \otimes w_{4(j+2)+1 \rightarrow 4(j+s+1)+1} ; \\
b_{j+2 s, 2 s+1}=w_{4(j+m)+2 \rightarrow 4(j+m+1)+2} \otimes w_{4(j+2)+1 \rightarrow 4(j+s)+2} ;
\end{gathered}
$$


HOCHSCHILD COHOMOLOGY RING FOR SELF-INJECTIVE ALGEBRAS OF TREE CLASS $E_{6}$. II. 127

$$
\begin{gathered}
b_{j+3 s, 2 s+1}=-w_{4(j+m)+3 \rightarrow 4(j+m+1)+2} \otimes w_{4(j+2)+1 \rightarrow 4 j+3} ; \\
b_{(j+1)_{s}, 5 s}=-w_{4(j+m+1) \rightarrow 4(j+m)} \otimes w_{4 j+3 \rightarrow 4(j+1)} .
\end{gathered}
$$

(15) If $r_{0}=10$ and $s>2$, then $\Omega^{10}\left(Y_{t}^{(19)}\right)$ is described with $(6 s \times 6 s)$-matrix with the following nonzero elements:

$$
\begin{gathered}
b_{j, 1}=\kappa^{\ell+1}\left(\alpha_{3(j+m-1)}\right) w_{4(j+m) \rightarrow 4(j+m)+3} \otimes e_{4 j} ; \\
b_{j+2 s, 1}=\kappa^{\ell+1}\left(\alpha_{3(j+m-1)}\right) w_{4(j+m)+1 \rightarrow 4(j+m)+3} \otimes w_{4 j \rightarrow 4(j+s)+1} ; \\
b_{j+4 s, 1}=-\kappa^{\ell+1}\left(\alpha_{3(j+m-1)}\right) w_{4(j+m)+2 \rightarrow 4(j+m)+3} \otimes w_{4 j \rightarrow 4(j+s)+2} ; \\
b_{(j+1)_{s}, s}=w_{4(j+m+1) \rightarrow 4(j+m+1)+2} \otimes w_{4(j+s)+1 \rightarrow 4(j+1)} ; \\
b_{s+(j+1)_{s}, s}=-w_{4(j+m+1)+1 \rightarrow 4(j+m+1)+2} \otimes w_{4(j+s)+1 \rightarrow 4(j+s+1)+1} ; \\
b_{(j+1)_{s}, 5 s}=-\kappa^{\ell+1}\left(\alpha_{3(j+m-1)}\right) w_{4(j+m+1) \rightarrow 4(j+m+2)} \otimes w_{4 j+3 \rightarrow 4(j+1)} ; \\
b_{2 s+(j+1)_{s}, 5 s}=-\kappa^{\ell+1}\left(\alpha_{3(j+m-1)}\right) w_{4(j+m+s+1)+1 \rightarrow 4(j+m+2)} \otimes w_{4 j+3 \rightarrow 4(j+1)+1} ; \\
b_{4 s+(j+1)_{s}, 5 s}=\kappa^{\ell+1}\left(\alpha_{3(j+m-1)}\right) w_{4(j+m+s+1)+2 \rightarrow 4(j+m+2)} \otimes w_{4 j+3 \rightarrow 4(j+1)+2} .
\end{gathered}
$$

(II) Represent an arbitrary $t_{0} \in \mathbb{N}$ in the form $t_{0}=11 \ell_{0}+r_{0}$, where $0 \leqslant r_{0} \leqslant 10$. Then $\Omega^{t_{0}}\left(Y_{t}^{(19)}\right)$ is a $\Omega^{r_{0}}\left(Y_{t}^{(19)}\right)$, whose left components twisted by $\sigma^{\ell_{0}}$, and coefficients multiplied by $(-1)^{\ell_{0}}$.

Proposition 36 (Translates for the case 20). (I) Let $r_{0} \in \mathbb{N}, r_{0}<11$. $r_{0}$-translates of the elements $Y_{t}^{(20)}$ are described by the following way.

(1) If $r_{0}=0$, then $\Omega^{0}\left(Y_{t}^{(20)}\right)$ is described with $(7 s \times 6 s)$-matrix with the following elements $b_{i j}$ : If $0 \leqslant j<s$, then

$$
b_{i j}=\left\{\begin{array}{l}
\kappa^{\ell}\left(\alpha_{3(j+m+4)}\right) w_{4(j+m) \rightarrow 4(j+m)+3} \otimes e_{4 j}, \quad i=j \\
0, \quad \text { otherwise. }
\end{array}\right.
$$

If $s \leqslant j<2 s$, then

$$
b_{i j}=\left\{\begin{array}{l}
-\kappa^{\ell}\left(\alpha_{3(j+m+4+1)}\right) w_{4(j+m+s)+1 \rightarrow 4(j+m+1)} \otimes e_{4(j+s)+1}, \quad i=j \\
0, \quad \text { otherwise. }
\end{array}\right.
$$

If $2 s \leqslant j<3 s$, then $b_{i j}=0$.

If $3 s \leqslant j<4 s$, then

$$
b_{i j}=\left\{\begin{array}{l}
w_{4(j+m+s)+2 \rightarrow 4(j+m+s+1)+1} \otimes e_{4(j+s)+2}, \quad i=j ; \\
0, \quad \text { otherwise. }
\end{array}\right.
$$

If $4 s \leqslant j<6 s$, then $b_{i j}=0$.

If $6 s \leqslant j<7 s$, then

$$
b_{i j}=\left\{\begin{array}{l}
w_{4(j+m)+3 \rightarrow 4(j+m+1)+2} \otimes e_{4 j+3}, \quad i=j-s \\
0, \quad \text { otherwise. }
\end{array}\right.
$$

(2) If $r_{0}=1$, then $\Omega^{1}\left(Y_{t}^{(20)}\right)$ is described with $(6 s \times 7 s)$-matrix with the following elements $b_{i j}$ : 
If $0 \leqslant j<s$, then

$$
b_{i j}=\left\{\begin{array}{l}
-\kappa^{\ell}\left(\alpha_{3(j+m+5)}\right) w_{4(j+m)+1 \rightarrow 4(j+m+1)} \otimes e_{4 j}, \quad i=j \\
0, \quad \text { otherwise. }
\end{array}\right.
$$

If $s \leqslant j<2 s$, then

$$
b_{i j}=\left\{\begin{array}{l}
-w_{4(j+m+s)+2 \rightarrow 4(j+m+s+1)+1} \otimes e_{4(j+s)+1}, \quad i=j+s \\
0, \quad \text { otherwise. }
\end{array}\right.
$$

If $2 s \leqslant j<3 s$, then $b_{i j}=0$.

If $3 s \leqslant j<4 s$, then

$$
b_{i j}=\left\{\begin{array}{l}
-w_{4(j+m)+3 \rightarrow 4(j+m+s+1)+2} \otimes e_{4(j+s)+2}, \quad i=j+s \\
0, \quad \text { otherwise. }
\end{array}\right.
$$

If $4 s \leqslant j<5 s$, then $b_{i j}=0$.

If $5 s \leqslant j<6 s$, then

$$
b_{i j}=\left\{\begin{array}{l}
\kappa^{\ell}\left(\alpha_{3(j+m+5)}\right) w_{4(j+m+1) \rightarrow 4(j+m+1)+3} \otimes e_{4 j+3}, \quad i=j+s \\
0, \quad \text { otherwise. }
\end{array}\right.
$$

(3) If $r_{0}=2$, then $\Omega^{2}\left(Y_{t}^{(20)}\right)$ is described with $(6 s \times 6 s)$-matrix with the following elements $b_{i j}$ : If $0 \leqslant j<s$, then

$$
b_{i j}= \begin{cases}\kappa_{1} w_{4(j+m)+2 \rightarrow 4(j+m+1)} \otimes w_{4 j \rightarrow 4 j+1}, & i=j+s \\ \kappa_{1} w_{4(j+m)+3 \rightarrow 4(j+m+1)} \otimes w_{4 j \rightarrow 4(j+1)}, & i=(j+1)_{s}, j=s-1 \\ 0, \quad \text { otherwise, } & \end{cases}
$$

where $\kappa_{1}=\kappa^{\ell}\left(\alpha_{3(j+m+5)}\right)$.

If $s \leqslant j<2 s-1$, then

$$
b_{i j}=\left\{\begin{array}{l}
w_{4(j+m)+3 \rightarrow 4(j+m+s+1)+1} \otimes w_{4(j+s)+1 \rightarrow 4(j+1)}, \quad i=(j+1)_{s} ; \\
0, \quad \text { otherwise. }
\end{array}\right.
$$

If $2 s-1 \leqslant j<3 s-1$, then $b_{i j}=0$.

If $3 s-1 \leqslant j<3 s$, then

$$
b_{i j}=\left\{\begin{array}{l}
w_{4(j+m)+3 \rightarrow 4(j+m+s+1)+1} \otimes w_{4(j+s)+1 \rightarrow 4(j+1)}, \quad i=(j+1)_{s} ; \\
0, \quad \text { otherwise. }
\end{array}\right.
$$

If $3 s \leqslant j<4 s-1$, then

$$
b_{i j}=\left\{\begin{array}{l}
w_{4(j+m)+3 \rightarrow 4(j+m+s+1)+2} \otimes w_{4(j+s)+2 \rightarrow 4(j+1)}, \quad i=(j+1)_{s} ; \\
0, \quad \text { otherwise. }
\end{array}\right.
$$

If $4 s-1 \leqslant j<5 s-1$, then $b_{i j}=0$. 
HOCHSCHILD COHOMOLOGY RING FOR SELF-INJECTIVE ALGEBRAS OF TREE CLASS E . II. 129 If $5 s-1 \leqslant j<5 s$, then

$$
b_{i j}=\left\{\begin{array}{l}
w_{4(j+m)+3 \rightarrow 4(j+m+s+1)+2} \otimes w_{4(j+s)+2 \rightarrow 4(j+1)}, \quad i=(j+1)_{s} ; \\
0, \quad \text { otherwise. }
\end{array}\right.
$$

If $5 s \leqslant j<6 s$, then

$$
b_{i j}=\left\{\begin{array}{l}
-\kappa^{\ell}\left(\alpha_{3(j+m+5)}\right) w_{4(j+m+s+1+s f(j, 6 s-1))+2 \rightarrow 4(j+m+1)+3} \otimes w_{4 j+3 \rightarrow 4(j+s+1+s f(j, 6 s-1))+1} \\
i=s+(j+1)_{s} \\
0, \quad \text { otherwise. }
\end{array}\right.
$$

(4) If $r_{0}=3$, then $\Omega^{3}\left(Y_{t}^{(20)}\right)$ is described with $(7 s \times 8 s)$-matrix with the following elements $b_{i j}$ : If $0 \leqslant j<s$, then

$$
b_{i j}=\left\{\begin{array}{l}
w_{4(j+m)+3 \rightarrow 4(j+m+1)+1} \otimes w_{4 j \rightarrow 4 j+1}, \quad i=j+2 s \\
0, \quad \text { otherwise. }
\end{array}\right.
$$

If $s \leqslant j<4 s$, then $b_{i j}=0$.

If $4 s \leqslant j<6 s$, then

$$
b_{i j}=\left\{\begin{array}{l}
\kappa^{\ell}\left(\alpha_{3(j+m+5)}\right) f_{1}(j, 5 s) w_{4(j+m+1)+2 \rightarrow 4(j+m+1)+3} \otimes w_{4 j+2 \rightarrow 4(j+1)}, \\
\quad i=(j+1)_{s}, j<5 s-1 \text { or } j=6 s-1 \\
0, \quad \text { otherwise. }
\end{array}\right.
$$

If $6 s \leqslant j<7 s$, then $b_{i j}=0$.

(5) If $r_{0}=4$, then $\Omega^{4}\left(Y_{t}^{(20)}\right)$ is described with $(6 s \times 9 s)$-matrix with the following elements $b_{i j}$ : If $0 \leqslant j<s$, then

$$
b_{i j}=\left\{\begin{array}{l}
\kappa_{1} w_{4(j+m+s)+1 \rightarrow 4(j+m)+3} \otimes w_{4 j \rightarrow 4 j+1}, \quad i=j+2 s \\
-\kappa_{1} w_{4(j+m+s)+2 \rightarrow 4(j+m)+3} \otimes w_{4 j \rightarrow 4 j+2}, \quad i=j+5 s \\
\kappa_{1} e_{4(j+m)+3} \otimes w_{4 j \rightarrow 4(j+1)}, \quad i=(j+1)_{s}, \quad j<s-1 \\
0, \quad \text { otherwise, }
\end{array}\right.
$$

where $\kappa_{1}=\kappa^{\ell}\left(\alpha_{3(j+m+4)}\right)$.

If $s \leqslant j<3 s$, then

$$
b_{i j}=\left\{\begin{array}{l}
w_{4(j+m)+3 \rightarrow 4(j+m+s+1)+2} \otimes w_{4(j+s)+1 \rightarrow 4(j+1)}, \quad i=(j+1)_{s}, j<2 s-1 \text { or } j=3 s-1 ; \\
0, \quad \text { otherwise. }
\end{array}\right.
$$

If $3 s \leqslant j<6 s$, then $b_{i j}=0$.

(6) If $r_{0}=5$, then $\Omega^{5}\left(Y_{t}^{(20)}\right)$ is described with $(8 s \times 8 s)$-matrix with the following elements $b_{i j}$ : If $s \leqslant j<2 s$, then

$$
b_{i j}= \begin{cases}w_{4(j+m+1)+1 \rightarrow 4(j+m+1)+2} \otimes w_{4 j \rightarrow 4(j+1)}, & i=(j+1)_{2 s} \\ w_{4(j+m+1) \rightarrow 4(j+m+1)+2} \otimes w_{4 j \rightarrow 4(j+s)+1}, & i=j+s \\ 0, \quad \text { otherwise. }\end{cases}
$$


If $2 s \leqslant j<4 s$, then

$$
b_{i j}=\left\{\begin{array}{c}
\kappa_{1} w_{4(j+m+1)+1 \rightarrow 4(j+m+1)+3} \otimes w_{4 j+1 \rightarrow 4(j+1)}, \\
i=(j+1)_{s}, j<3 s-1 \text { or } j=4 s-1 ; \\
\kappa_{1} w_{4(j+m+s+1)+1 \rightarrow 4(j+m+1)+3} \otimes w_{4 j+1 \rightarrow 4(j+1)}, \\
i=s+(j+1)_{s}, j<3 s-1 \text { or } j=4 s-1 ; \\
0, \quad \text { otherwise, }
\end{array}\right.
$$

where $\kappa_{1}=\kappa^{\ell+1}\left(\alpha_{3(j+m-1)}\right)$.

If $4 s \leqslant j<6 s$, then

$b_{i j}=\left\{\begin{array}{l}\kappa^{\ell+1}\left(\alpha_{3(j+m)}\right) e_{4(j+m+2)} \otimes w_{4 j+2 \rightarrow 4(j+1)+1}, \quad i=2 s+(j+1)_{s}, j<5 s-1 \text { or } j=6 s-1 ; \\ 0, \quad \text { otherwise. }\end{array}\right.$

If $6 s \leqslant j<8 s$, then $b_{i j}=0$.

(7) If $r_{0}=6$, then $\Omega^{6}\left(Y_{t}^{(20)}\right)$ is described with $(9 s \times 9 s)$-matrix with the following elements $b_{i j}$ : If $0 \leqslant j<s$, then

$$
b_{i j}=\left\{\begin{array}{l}
-\kappa^{\ell+1}\left(\alpha_{3(j+m-2)}\right) w_{4(j+m)+1 \rightarrow 4(j+m)+3} \otimes w_{4 j \rightarrow 4 j+1}, \quad i=j+s \\
0, \quad \text { otherwise. }
\end{array}\right.
$$

If $s \leqslant j<2 s-1$, then

$$
b_{i j}=\left\{\begin{array}{l}
\kappa^{\ell+1}\left(\alpha_{3(j+m-1)}\right) e_{4(j+m+1)} \otimes w_{4 j \rightarrow 4(j+1)}, \quad i=(j+1)_{s} \\
0, \quad \text { otherwise. }
\end{array}\right.
$$

If $2 s-1 \leqslant j<2 s$, then $b_{i j}=0$.

If $2 s \leqslant j<4 s$, then

$b_{i j}=\left\{\begin{array}{l}w_{4(j+m+1) \rightarrow 4(j+m+s+1)+1} \otimes w_{4 j+1 \rightarrow 4(j+1)}, \quad i=(j+1)_{s}, j<3 s-1 \text { or } j=4 s-1 \\ 0, \quad \text { otherwise. }\end{array}\right.$

If $4 s \leqslant j<5 s-1$, then

$$
b_{i j}=\left\{\begin{array}{l}
e_{4(j+m+1)+1} \otimes w_{4 j+2 \rightarrow 4(j+1)+1}, \quad i=s+(j+1)_{s} \\
0, \quad \text { otherwise. }
\end{array}\right.
$$

If $5 s-1 \leqslant j<7 s-1$, then $b_{i j}=0$.

If $7 s-1 \leqslant j<7 s$, then

$$
b_{i j}=\left\{\begin{array}{l}
e_{4(j+m+s+1)+1} \otimes w_{4(j+s)+2 \rightarrow 4(j+s+1)+1}, \quad i=s+(j+1)_{s} \\
0, \quad \text { otherwise. }
\end{array}\right.
$$

If $7 s \leqslant j<9 s$, then $b_{i j}=0$.

(8) If $r_{0}=7$, then $\Omega^{7}\left(Y_{t}^{(20)}\right)$ is described with $(8 s \times 8 s)$-matrix with the following elements $b_{i j}$ : 
HOCHSCHILD COHOMOLOGY RING FOR SELF-INJECTIVE ALGEBRAS OF TREE CLASS $E_{6}$. II. 131 If $0 \leqslant j<s$, then

$$
b_{i j}=\left\{\begin{array}{l}
-w_{4(j+m)+3 \rightarrow 4(j+m+1)+1} \otimes w_{4 j \rightarrow 4 j+1}, \quad i=j+2 s \\
-w_{4(j+m+1) \rightarrow 4(j+m+1)+1} \otimes w_{4 j \rightarrow 4 j+2}, \quad i=j+4 s \\
-w_{4(j+m+1) \rightarrow 4(j+m+1)+1} \otimes w_{4 j \rightarrow 4(j+s)+2}, \quad i=j+5 s \\
0, \quad \text { otherwise. }
\end{array}\right.
$$

If $s \leqslant j<2 s$, then $b_{i j}=0$.

If $2 s \leqslant j<4 s$, then

$$
b_{i j}=\left\{\begin{array}{c}
\kappa^{\ell+1}\left(\alpha_{3(j+m)}\right) w_{4(j+m+1)+2 \rightarrow 4(j+m+2)} \otimes w_{4 j+1 \rightarrow 4(j+1)}, \\
i=(j+1)_{s}, j<3 s-1 \text { or } j=4 s-1 \\
0, \quad \text { otherwise. }
\end{array}\right.
$$

If $4 s \leqslant j<8 s$, then $b_{i j}=0$.

(9) If $r_{0}=8$, then $\Omega^{8}\left(Y_{t}^{(20)}\right)$ is described with $(9 s \times 6 s)$-matrix with the following elements $b_{i j}$ : If $0 \leqslant j<s$, then

$$
b_{i j}=\left\{\begin{array}{l}
-\kappa_{1} w_{4(j+m)+2 \rightarrow 4(j+m+1)} \otimes w_{4 j \rightarrow 4 j+1}, \quad i=j+s \\
-\kappa_{1} w_{4(j+m)+1 \rightarrow 4(j+m+1)} \otimes w_{4 j \rightarrow 4(j+s)+2}, \quad i=j+4 s \\
\kappa_{1} w_{4(j+m)+3 \rightarrow 4(j+m+1)} \otimes w_{4 j \rightarrow 4(j+1)}, \quad i=(j+1)_{s}, \quad j=s-1 \\
0, \quad \text { otherwise, }
\end{array}\right.
$$

where $\kappa_{1}=\kappa^{\ell+1}\left(\alpha_{3(j+m-1)}\right)$.

If $s \leqslant j<2 s-1$, then

$$
b_{i j}=\left\{\begin{array}{l}
w_{4(j+m)+3 \rightarrow 4(j+m+s+1)+1} \otimes w_{4(j+s)+1 \rightarrow 4(j+1)}, \quad i=(j+1)_{s} ; \\
0, \quad \text { otherwise. }
\end{array}\right.
$$

If $2 s-1 \leqslant j<4 s-1$, then $b_{i j}=0$.

If $4 s-1 \leqslant j<4 s$, then

$$
b_{i j}=\left\{\begin{array}{l}
w_{4(j+m)+3 \rightarrow 4(j+m+1)+1} \otimes w_{4 j+1 \rightarrow 4(j+1),} \quad i=(j+1)_{s} ; \\
0, \quad \text { otherwise. }
\end{array}\right.
$$

If $4 s \leqslant j<7 s$, then $b_{i j}=0$.

If $7 s \leqslant j<8 s$, then

$$
b_{i j}=\left\{\begin{array}{l}
\kappa_{1} w_{4(j+m)+3 \rightarrow 4(j+m+1)+3} \otimes w_{4 j+3 \rightarrow 4(j+1)}, \quad i=(j+1)_{s} ; \\
\kappa_{1} w_{4(j+m+1+s f(j, 8 s-1))+2 \rightarrow 4(j+m+1)+3} \otimes w_{4 j+3 \rightarrow 4(j+1+s f(j, 8 s-1))+1,} \quad i=2 s+(j+1)_{s} ; \\
\kappa_{1} w_{4(j+m+1+s f(j, 8 s-1))+1 \rightarrow 4(j+m+1)+3} \otimes w_{4 j+3 \rightarrow 4(j+s+1+s f(j, 8 s-1))+2,} i=3 s+(j+1)_{s} ; \\
0, \quad \text { otherwise, }
\end{array}\right.
$$

where $\kappa_{1}=-\kappa^{\ell+1}\left(\alpha_{3(j+m-1)}\right)$.

If $8 s \leqslant j<9 s$, then $b_{i j}=0$. 
(10) If $r_{0}=9$, then $\Omega^{9}\left(Y_{t}^{(20)}\right)$ is described with $(8 s \times 7 s)$-matrix with the following elements $b_{i j}$ :

If $0 \leqslant j<s$, then

$$
b_{i j}=\left\{\begin{array}{l}
w_{4(j+m)+3 \rightarrow 4(j+m+1)+2} \otimes e_{4 j}, \quad i=j ; \\
0, \quad \text { otherwise. }
\end{array}\right.
$$

If $s \leqslant j<3 s-1$, then $b_{i j}=0$.

If $3 s-1 \leqslant j<4 s-1$, then

$$
b_{i j}=\left\{\begin{array}{l}
-\kappa^{\ell+1}\left(\alpha_{3(j+m-1)}\right) e_{4(j+m+1)+3} \otimes w_{4 j+1 \rightarrow 4(j+1)}, \quad i=(j+1)_{s} \\
0, \quad \text { otherwise. }
\end{array}\right.
$$

If $4 s-1 \leqslant j<5 s$, then $b_{i j}=0$.

If $5 s \leqslant j<6 s$, then

$$
b_{i j}=\left\{\begin{array}{l}
\kappa^{\ell+1}\left(\alpha_{3(j+m)}\right) w_{4(j+m+1)+3 \rightarrow 4(j+m+2)} \otimes w_{4 j+2 \rightarrow 4(j+1)}, \quad i=(j+1)_{s} ; \\
0, \quad \text { otherwise. }
\end{array}\right.
$$

If $6 s \leqslant j<7 s-1$, then

$$
b_{i j}=\left\{\begin{array}{l}
-w_{4(j+m+1)+3 \rightarrow 4(j+m+2)+1} \otimes w_{4 j+3 \rightarrow 4(j+1)}, \quad i=(j+1)_{s} \\
0, \quad \text { otherwise. }
\end{array}\right.
$$

If $7 s-1 \leqslant j<7 s$, then

$$
b_{i j}=\left\{\begin{array}{l}
-w_{4(j+m+2) \rightarrow 4(j+m+2)+1} \otimes w_{4 j+3 \rightarrow 4(j+1)+1}, \quad i=2 s+(j+1)_{s} \\
-e_{4(j+m+2)+1} \otimes w_{4 j+3 \rightarrow 4(j+s+1)+2}, \quad i=3 s+(j+1)_{s} \\
0, \quad \text { otherwise. }
\end{array}\right.
$$

If $7 s \leqslant j<8 s$, then $b_{i j}=0$.

(11) If $r_{0}=10$, then $\Omega^{10}\left(Y_{t}^{(20)}\right)$ is described with $(6 s \times 6 s)$-matrix with the following elements $b_{i j}$ :

If $0 \leqslant j<s$, then

$$
b_{i j}=\left\{\begin{array}{l}
\kappa_{1} w_{4(j+m) \rightarrow 4(j+m)+3} \otimes e_{4 j}, \quad i=j ; \\
-\kappa_{1} w_{4(j+m+s)+1 \rightarrow 4(j+m)+3} \otimes w_{4 j \rightarrow 4 j+1}, \quad i=j+s \\
\kappa_{1} w_{4(j+m)+1 \rightarrow 4(j+m)+3} \otimes w_{4 j \rightarrow 4(j+s)+1}, \quad i=j+2 s \\
\kappa_{1} w_{4(j+m+s)+2 \rightarrow 4(j+m)+3} \otimes w_{4 j \rightarrow 4 j+2}, \quad i=j+3 s \\
-\kappa_{1} w_{4(j+m)+2 \rightarrow 4(j+m)+3} \otimes w_{4 j \rightarrow 4(j+s)+2}, \quad i=j+4 s \\
\kappa_{1} e_{4(j+m)+3} \otimes w_{4 j \rightarrow 4 j+3}, \quad i=j+5 s \\
0, \quad \text { otherwise, }
\end{array}\right.
$$

where $\kappa_{1}=\kappa^{\ell+1}\left(\alpha_{3(j+m-2)}\right)$.

If $s \leqslant j<2 s-1$, then $b_{i j}=0$. 
HOCHSCHILD COHOMOLOGY RING FOR SELF-INJECTIVE ALGEBRAS OF TREE CLASS $E_{6}$. II. 133 If $2 s-1 \leqslant j<2 s$, then

$$
b_{i j}=\left\{\begin{array}{l}
-w_{4(j+m+1) \rightarrow 4(j+m+s+1)+2} \otimes w_{4(j+s)+1 \rightarrow 4(j+1)}, \quad i=(j+1)_{s} \\
0, \quad \text { otherwise. }
\end{array}\right.
$$

If $2 s \leqslant j<5 s-1$, then $b_{i j}=0$.

If $5 s-1 \leqslant j<5 s$, then

$$
b_{i j}=\left\{\begin{array}{l}
w_{4(j+m+1) \rightarrow 4(j+m+1)+1} \otimes w_{4(j+s)+2 \rightarrow 4(j+1)}, \quad i=(j+1)_{s} \\
-e_{4(j+m+1)+1} \otimes w_{4(j+s)+2 \rightarrow 4(j+s+1)+1}, \quad i=s+(j+1)_{s} \\
0, \quad \text { otherwise. }
\end{array}\right.
$$

If $5 s \leqslant j<6 s-1$, then $b_{i j}=0$.

If $6 s-1 \leqslant j<6 s$, then

$$
b_{i j}= \begin{cases}\kappa_{1} w_{4(j+m+1)+1 \rightarrow 4(j+m+2)} \otimes w_{4 j+3 \rightarrow 4(j+s+1)+1}, & i=2 s+(j+1)_{s} \\ \kappa_{1} w_{4(j+m+s+1)+2 \rightarrow 4(j+m+2)} \otimes w_{4 j+3 \rightarrow 4(j+1)+2}, & i=3 s+(j+1)_{s} \\ -\kappa_{1} w_{4(j+m+1)+2 \rightarrow 4(j+m+2)} \otimes w_{4 j+3 \rightarrow 4(j+s+1)+2}, & i=4 s+(j+1)_{s} \\ \kappa_{1} w_{4(j+m+1)+3 \rightarrow 4(j+m+2)} \otimes w_{4 j+3 \rightarrow 4(j+1)+3}, \quad i=5 s+(j+1)_{s} \\ 0, \quad \text { otherwise, }\end{cases}
$$

where $\kappa_{1}=-f_{2}(s, 1) \kappa^{\ell+1}\left(\alpha_{3(j+m+1)}\right)$.

(II) Represent an arbitrary $t_{0} \in \mathbb{N}$ in the form $t_{0}=11 \ell_{0}+r_{0}$, where $0 \leqslant r_{0} \leqslant 10$. Then $\Omega^{t_{0}}\left(Y_{t}^{(20)}\right)$ is a $\Omega^{r_{0}}\left(Y_{t}^{(20)}\right)$, whose left components twisted by $\sigma^{\ell_{0}}$.

Proposition 37 (Translates for the case 21). (I) Let $r_{0} \in \mathbb{N}, r_{0}<11$. Denote by $\kappa_{0}=$ $\kappa^{\ell}\left(\gamma_{4}\right) \kappa^{\ell}\left(\gamma_{5}\right) \kappa^{\ell}\left(\gamma_{6}\right)-\kappa^{\ell}\left(\alpha_{15}\right)$. Then $r_{0}$-translates of the elements $Y_{t}^{(21)}$ are described by the following way.

(1) If $r_{0}=0$, then $\Omega^{0}\left(Y_{t}^{(21)}\right)$ is described with $(6 s \times 6 s)$-matrix with one nonzero element that is of the following form:

$$
b_{0,0}=-\kappa^{\ell}\left(\alpha_{15}\right) w_{4(j+m) \rightarrow 4(j+m+1)} \otimes e_{4 j} .
$$

(2) If $r_{0}=1$, then $\Omega^{1}\left(Y_{t}^{(21)}\right)$ is described with $(6 s \times 7 s)$-matrix with one nonzero element that is of the following form:

$$
b_{(j+1)_{s},(1)_{s}}=f_{2}(s, 1) \kappa_{0} w_{4(j+m+1+f(s, 1))+1 \rightarrow 4(j+m+2)} \otimes w_{4 j \rightarrow 4(j+1)} .
$$

(3) If $r_{0}=2$, then $\Omega^{2}\left(Y_{t}^{(21)}\right)$ is described with $(7 s \times 6 s)$-matrix with one nonzero element that is of the following form:

$$
b_{(j+1)_{s}, 1}=f_{2}(s, 1) \kappa^{\ell+1}\left(\alpha_{3(j+m-1)}\right) \kappa_{0} w_{4(j+m)+3 \rightarrow 4(j+m+1)+1} \otimes w_{4 j \rightarrow 4(j+1)} .
$$

(4) If $r_{0}=3$, then $\Omega^{3}\left(Y_{t}^{(21)}\right)$ is described with $(6 s \times 8 s)$-matrix with one nonzero element that is of the following form:

$$
b_{(j+1)_{s},(1)_{s}}=-\kappa_{0} w_{4(j+m+1+f(s, 1))+2 \rightarrow 4(j+m+1)+3} \otimes w_{4 j \rightarrow 4(j+1)} .
$$


(5) If $r_{0}=4$, then $\Omega^{4}\left(Y_{t}^{(21)}\right)$ is described with $(8 s \times 9 s)$-matrix with one nonzero element that is of the following form:

$$
b_{(j+1)_{s}, 1}=-\kappa^{\ell+1}\left(\alpha_{3(j+m-1)}\right) \kappa_{0} w_{4(j+m)+3 \rightarrow 4(j+m+1)+2} \otimes w_{4 j \rightarrow 4(j+1)} .
$$

(6) If $r_{0}=5$, then $\Omega^{5}\left(Y_{t}^{(21)}\right)$ is described with $(9 s \times 8 s)$-matrix with the following two nonzero elements:

$$
\begin{aligned}
b_{(j+1)_{s}+f(s, 1),(1)_{s}} & =f_{2}(s, 1) \kappa_{0} w_{4(j+m+1)+1 \rightarrow 4(j+m+1)+3} \otimes w_{4 j \rightarrow 4(j+1)} ; \\
b_{s+(j+1)_{s}-f(s, 1),(1)_{s}} & =f_{2}(s, 1) \kappa_{0} w_{4(j+m+s+1)+1 \rightarrow 4(j+m+1)+3} \otimes w_{4 j \rightarrow 4(j+1)} .
\end{aligned}
$$

(7) If $r_{0}=6$, then $\Omega^{6}\left(Y_{t}^{(21)}\right)$ is described with $(8 s \times 9 s)$-matrix with one nonzero element that is of the following form:

$$
b_{(j+1)_{s},(1)_{s}}=f_{2}(s, 1) \kappa^{\ell+1}\left(\alpha_{3(j+m-1)}\right) \kappa_{0} w_{4(j+m+1) \rightarrow 4(j+m+1)+1} \otimes w_{4 j \rightarrow 4(j+1)} .
$$

(8) If $r_{0}=7$, then $\Omega^{7}\left(Y_{t}^{(21)}\right)$ is described with $(9 s \times 8 s)$-matrix with one nonzero element that is of the following form:

$$
b_{(j+1)_{s},(1)_{s}}=f_{2}(s, 1) \kappa^{\ell+1}\left(\gamma_{j+m-1}\right) \kappa_{0} w_{4(j+m+1+f(s, 1))+2 \rightarrow 4(j+m+2)} \otimes w_{4 j \rightarrow 4(j+1)} .
$$

(9) If $r_{0}=8$, then $\Omega^{8}\left(Y_{t}^{(21)}\right)$ is described with $(8 s \times 6 s)$-matrix with one nonzero element that is of the following form:

$$
b_{(j+1)_{s}, 1}=f_{2}(s, 1) \kappa^{\ell+1}\left(\alpha_{3(j+m-2)}\right) \kappa_{0} w_{4(j+m)+3 \rightarrow 4(j+m+1)+2} \otimes w_{4 j \rightarrow 4(j+1)} .
$$

(10) If $r_{0}=9$, then $\Omega^{9}\left(Y_{t}^{(21)}\right)$ is described with $(6 s \times 7 s)$-matrix with one nonzero element that is of the following form:

$$
b_{j,(1)_{s}}=-f_{2}(s, 1) \kappa^{\ell+1}\left(\gamma_{j+m-2}\right) \kappa_{0} w_{4(j+m)+3 \rightarrow 4(j+m+1)+3} \otimes e_{4 j} .
$$

(11) If $r_{0}=10$, then $\Omega^{10}\left(Y_{t}^{(21)}\right)$ is described with $(7 s \times 6 s)$-matrix with one nonzero element that is of the following form:

$$
b_{(j+1)_{s}, 0}=-\kappa^{\ell+1}\left(\gamma_{j+m-2}\right) \kappa_{0} w_{4(j+m+1) \rightarrow 4(j+m+1)+3} \otimes w_{4 j \rightarrow 4(j+1)} .
$$

(II) Represent an arbitrary $t_{0} \in \mathbb{N}$ in the form $t_{0}=11 \ell_{0}+r_{0}$, where $0 \leqslant r_{0} \leqslant 10$. Then $\Omega^{t_{0}}\left(Y_{t}^{(21)}\right)$ is a $\Omega^{r_{0}}\left(Y_{t}^{(21)}\right)$, whose left components twisted by $\sigma^{\ell_{0}}$, and coefficients multiplied by $(-1)^{\ell_{0}}$.

Proposition 38 (Translates for the case 22). (I) Let $r_{0} \in \mathbb{N}, r_{0}<11$. $r_{0}$-translates of the elements $Y_{t}^{(22)}$ are described by the following way.

(1) If $r_{0}=0$, then $\Omega^{0}\left(Y_{t}^{(22)}\right)$ is described with $(6 s \times 6 s)$-matrix with the following elements $b_{i j}$ : If $0 \leqslant j<s$, then

If $s \leqslant j<5 s$, then $b_{i j}=0$.

$$
b_{i j}=\left\{\begin{array}{l}
\kappa^{\ell}\left(\alpha_{3(j+m+5)}\right) e_{4(j+m)} \otimes e_{4 j}, \quad i=j \\
0, \quad \text { otherwise. }
\end{array}\right.
$$

If $5 s \leqslant j<6 s$, then

$$
b_{i j}=\left\{\begin{array}{l}
-\kappa^{\ell}\left(\alpha_{3(j+m+5)}\right) e_{4(j+m)+3} \otimes e_{4 j+3}, \quad i=j \\
0, \quad \text { otherwise. }
\end{array}\right.
$$

(2) If $r_{0}=1$, then $\Omega^{1}\left(Y_{t}^{(22)}\right)$ is described with $(6 s \times 7 s)$-matrix with the following elements $b_{i j}$ : 
HOCHSCHILD COHOMOLOGY RING FOR SELF-INJECTIVE ALGEBRAS OF TREE CLASS E $E_{6}$ II. 135 If $0 \leqslant j<s$, then

$$
b_{i j}=\left\{\begin{array}{l}
\kappa_{1} w_{4(j+m)+1 \rightarrow 4(j+m+1)} \otimes e_{4 j}, \quad i=j \\
\kappa_{1} w_{4(j+m)+2 \rightarrow 4(j+m+1)} \otimes w_{4 j \rightarrow 4 j+1}, \quad i=j+2 s \\
\kappa_{1} w_{4(j+m)+3 \rightarrow 4(j+m+1)} \otimes w_{4 j \rightarrow 4 j+2}, \quad i=j+4 s \\
-\kappa_{1} e_{4(j+m+1)} \otimes w_{4 j \rightarrow 4 j+3}, \quad i=j+6 s \\
0, \quad \text { otherwise, }
\end{array}\right.
$$

where $\kappa_{1}=-\kappa^{\ell}\left(\alpha_{3(j+m+6)}\right)$.

If $s \leqslant j<3 s$, then

$$
b_{i j}=\left\{\begin{array}{l}
-e_{4(j+m+1)+1} \otimes w_{4(j+s)+1 \rightarrow 4(j+1)}, \quad i=(j+1)_{2 s} ; \\
w_{4(j+m)+3 \rightarrow 4(j+m+1)+1} \otimes w_{4(j+s)+1 \rightarrow 4(j+s)+2}, \quad i=j+3 s \\
0, \quad \text { otherwise. }
\end{array}\right.
$$

If $3 s \leqslant j<5 s$, then

$$
b_{i j}=\left\{\begin{array}{l}
-w_{4(j+m+1)+1 \rightarrow 4(j+m+1)+2} \otimes w_{4(j+s)+2 \rightarrow 4(j+1)}, \quad i=(j+1)_{2 s} \\
w_{4(j+m)+3 \rightarrow 4(j+m+1)+2} \otimes e_{4(j+s)+2}, \quad i=j+s \\
0, \quad \text { otherwise. }
\end{array}\right.
$$

If $5 s \leqslant j<6 s$, then

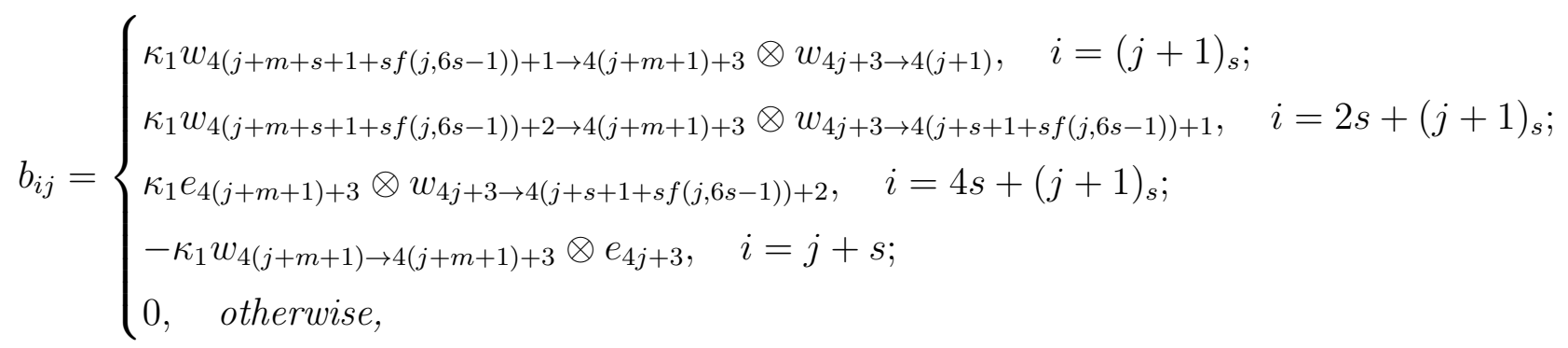

where $\kappa_{1}=-\kappa^{\ell}\left(\alpha_{3(j+m+6)}\right)$.

(3) If $r_{0}=2$, then $\Omega^{2}\left(Y_{t}^{(22)}\right)$ is described with $(7 s \times 6 s)$-matrix with the following elements $b_{i j}$ : If $0 \leqslant j<s$, then

$$
b_{i j}=\left\{\begin{array}{l}
e_{4(j+m+s)+1} \otimes w_{4 j \rightarrow 4 j+2}, \quad i=j+3 s \\
0, \quad \text { otherwise }
\end{array}\right.
$$

If $s \leqslant j<2 s$, then

$$
b_{i j}=\left\{\begin{array}{l}
-w_{4(j+m)-1 \rightarrow 4(j+m+s)+1} \otimes e_{4 j}, \quad i=j-s \\
e_{4(j+m+s)+1} \otimes w_{4 j \rightarrow 4 j+2}, \quad i=j+3 s \\
0, \quad \text { otherwise. }
\end{array}\right.
$$

If $2 s \leqslant j<4 s$, then $b_{i j}=0$. 
If $4 s \leqslant j<6 s$, then

$$
b_{i j}=\left\{\begin{array}{l}
\kappa_{1} w_{4(j+m+s)+1 \rightarrow 4(j+m)+3} \otimes e_{4 j+2}, \quad i=j-s ; \\
-\kappa_{1} e_{4(j+m)+3} \otimes w_{4 j+2 \rightarrow 4(j+1)}, \quad i=(j+1)_{s}, j<5 s-1 \text { or } j=6 s-1 ; \\
0, \quad \text { otherwise, }
\end{array}\right.
$$

where $\kappa_{1}=\kappa^{\ell}\left(\alpha_{3(j+m+5)}\right) f_{1}(j, 5 s)$.

If $6 s \leqslant j<7 s$, then

$$
b_{i j}=\left\{\begin{array}{l}
-\kappa^{\ell+1}\left(\alpha_{3(j+m)}\right) e_{4(j+m+1)} \otimes e_{4 j+3}, \quad i=j-s \\
0, \quad \text { otherwise. }
\end{array}\right.
$$

(4) If $r_{0}=3$, then $\Omega^{3}\left(Y_{t}^{(22)}\right)$ is described with $(6 s \times 8 s)$-matrix with the following elements $b_{i j}$ : If $0 \leqslant j<s$, then

$$
b_{i j}=\left\{\begin{array}{l}
\kappa_{1} w_{4(j+m)+2 \rightarrow 4(j+m)+3} \otimes e_{4 j}, \quad i=j \\
\kappa_{1} e_{4(j+m)+3} \otimes w_{4 j \rightarrow 4 j+1}, \quad i=j+2 s \\
0, \quad \text { otherwise }
\end{array}\right.
$$

where $\kappa_{1}=\kappa^{\ell}\left(\alpha_{3(j+m+5)}\right)$.

If $s \leqslant j<3 s$, then

$$
b_{i j}=\left\{\begin{array}{l}
-f_{2}\left((j)_{s}, s-1\right) f_{1}(j, 2 s) e_{4(j+m+1)+2} \otimes w_{4(j+s)+1 \rightarrow 4(j+1)}, \quad i=(j+1)_{2 s} \\
w_{4(j+m+1) \rightarrow 4(j+m+1)+2} \otimes w_{4(j+s)+1 \rightarrow 4(j+s)+2}, \quad i=j+3 s \\
0, \quad \text { otherwise. }
\end{array}\right.
$$

If $3 s \leqslant j<5 s$, then

$$
b_{i j}=\left\{\begin{array}{l}
w_{4(j+m+1) \rightarrow 4(j+m+s+1)+1} \otimes e_{4(j+s)+2}, \quad i=j+s \\
0, \quad \text { otherwise }
\end{array}\right.
$$

If $5 s \leqslant j<6 s$, then

$$
b_{i j}=\left\{\begin{array}{l}
-\kappa^{\ell+1}\left(\alpha_{3(j+m+1)}\right) w_{4(j+m+s+1)+2 \rightarrow 4(j+m+2)} \otimes w_{4 j+3 \rightarrow 4(j+1)} \\
i=(j+1)_{s}, j<6 s-1 ; \\
-\kappa^{\ell+1}\left(\alpha_{3(j+m+1)}\right) w_{4(j+m+1)+3 \rightarrow 4(j+m+2)} \otimes w_{4 j+3 \rightarrow 4(j+s+1)+1} \\
i=2 s+(j+1)_{s}, j<6 s-1 ; \\
\kappa^{\ell+1}\left(\alpha_{3(j+m+1)}\right) e_{4(j+m+2)} \otimes w_{4 j+3 \rightarrow 4(j+s+1+s f(j, 6 s-1))+2}, \quad i=4 s+(j+1)_{s} ; \\
-\kappa^{\ell+1}\left(\alpha_{3(j+m+1)}\right) w_{4(j+m+s+1)+1 \rightarrow 4(j+m+2)} \otimes e_{4 j+3}, \quad i=j+s \\
0, \quad \text { otherwise. }
\end{array}\right.
$$

(5) If $r_{0}=4$, then $\Omega^{4}\left(Y_{t}^{(22)}\right)$ is described with $(8 s \times 9 s)$-matrix with the following elements $b_{i j}$ : 
HOCHSCHILD COHOMOLOGY RING FOR SELF-INJECTIVE ALGEBRAS OF TREE CLASS E. II. 137 If $0 \leqslant j<s$, then

$$
b_{i j}=\left\{\begin{array}{l}
-w_{4(j+m) \rightarrow 4(j+m+s)+2} \otimes e_{4 j}, \quad i=j+s \\
-w_{4(j+m+s)+1 \rightarrow 4(j+m+s)+2} \otimes w_{4 j \rightarrow 4 j+1}, \quad i=j+2 s \\
e_{4(j+m+s)+2} \otimes w_{4 j \rightarrow 4 j+2}, \quad i=j+5 s \\
0, \quad \text { otherwise. }
\end{array}\right.
$$

If $s \leqslant j<2 s$, then

$$
b_{i j}=\left\{\begin{array}{l}
-w_{4(j+m)-1 \rightarrow 4(j+m+s)+2} \otimes e_{4 j}, \quad i=j-s \\
-w_{4(j+m) \rightarrow 4(j+m+s)+2} \otimes e_{4 j}, \quad i=j ; \\
w_{4(j+m+s)+1 \rightarrow 4(j+m+s)+2} \otimes w_{4 j \rightarrow 4 j+1}, \quad i=j+2 s \\
-e_{4(j+m+s)+2} \otimes w_{4 j \rightarrow 4 j+2}, \quad i=j+6 s \\
0, \quad \text { otherwise. }
\end{array}\right.
$$

If $2 s \leqslant j<4 s$, then

$b_{i j}=\left\{\begin{array}{l}-\kappa^{\ell+1}\left(\alpha_{3(j+m-1)}\right) e_{4(j+m)+3} \otimes w_{4 j+1 \rightarrow 4(j+1)}, \quad i=(j+1)_{s}, j<3 s-1 \text { or } j=4 s-1 ; \\ 0, \quad \text { otherwise. }\end{array}\right.$

If $4 s \leqslant j<5 s$, then

$$
b_{i j}=\left\{\begin{array}{l}
-\kappa_{1} w_{4(j+m)+1 \rightarrow 4(j+m+1)} \otimes e_{4 j+2}, \quad i=j \\
\kappa_{1} e_{4(j+m+1)} \otimes w_{4 j+2 \rightarrow 4(j+1)}, \quad i=s+(j+1)_{s}, \quad j<5 s-1 \\
0, \quad \text { otherwise, }
\end{array}\right.
$$

where $\kappa_{1}=-\kappa^{\ell+1}\left(\alpha_{3(j+m)}\right)$.

If $5 s \leqslant j<6 s$, then

$$
b_{i j}=\left\{\begin{array}{l}
-\kappa_{1} w_{4(j+m+s)+2 \rightarrow 4(j+m+1)} \otimes e_{4 j+2}, \quad i=j+2 s ; \\
\kappa_{1} w_{4(j+m)+3 \rightarrow 4(j+m+1)} \otimes w_{4 j+2 \rightarrow 4(j+1)}, \quad i=(j+1)_{s}, \quad j=6 s-1 ; \\
\kappa_{1} e_{4(j+m+1)} \otimes w_{4 j+2 \rightarrow 4(j+1)}, \quad i=s+(j+1)_{s}, \quad j=6 s-1 ; \\
0, \quad \text { otherwise, }
\end{array}\right.
$$

where $\kappa_{1}=-\kappa^{\ell+1}\left(\alpha_{3(j+m)}\right)$.

If $6 s \leqslant j<7 s-1$, then

$$
b_{i j}=\left\{\begin{array}{l}
w_{4(j+m+1) \rightarrow 4(j+m+s+1)+1} \otimes w_{4 j+3 \rightarrow 4(j+1)}, \quad i=s+(j+1)_{s} \\
0, \quad \text { otherwise. }
\end{array}\right.
$$

If $7 s-1 \leqslant j<7 s$, then $b_{i j}=0$. 
If $7 s \leqslant j<8 s$, then

$$
b_{i j}=\left\{\begin{array}{l}
-w_{4(j+m)+3 \rightarrow 4(j+m+s+1)+1} \otimes e_{4 j+3}, \quad i=j+s \\
w_{4(j+m+1) \rightarrow 4(j+m+s+1)+1} \otimes w_{4 j+3 \rightarrow 4(j+1)}, \quad i=s+(j+1)_{s}, j=8 s-1 \\
0, \quad \text { otherwise. }
\end{array}\right.
$$

(6) If $r_{0}=5$, then $\Omega^{5}\left(Y_{t}^{(22)}\right)$ is described with $(9 s \times 8 s)$-matrix with the following elements $b_{i j}$ : If $0 \leqslant j<s$, then

$$
b_{i j}=\left\{\begin{array}{l}
\kappa_{1} w_{4(j+m)+1 \rightarrow 4(j+m)+3} \otimes e_{4 j}, \quad i=j \\
\kappa_{1} w_{4(j+m+s)+1 \rightarrow 4(j+m)+3} \otimes e_{4 j}, \quad i=j+s \\
-\kappa_{1} e_{4(j+m)+3} \otimes w_{4 j \rightarrow 4 j+2}, \quad i=j+4 s \\
-\kappa_{1} e_{4(j+m)+3} \otimes w_{4 j \rightarrow 4(j+s)+2}, \quad i=j+5 s \\
0, \quad \text { otherwise, }
\end{array}\right.
$$

where $\kappa_{1}=\kappa^{\ell+1}\left(\alpha_{3(j+m-1)}\right)$.

If $s \leqslant j<2 s$, then

$$
b_{i j}=\left\{\begin{array}{l}
-\kappa_{1} w_{4(j+m+s)+1 \rightarrow 4(j+m+1)} \otimes e_{4 j}, \quad i=j-s \\
\kappa_{1} e_{4(j+m+1)} \otimes w_{4 j \rightarrow 4(j+s)+1}, \quad i=j+s \\
-\kappa_{1} e_{4(j+m+1)} \otimes w_{4 j \rightarrow 4 j+1}, \quad i=j+2 s \\
0, \quad \text { otherwise, }
\end{array}\right.
$$

where $\kappa_{1}=-\kappa^{\ell+1}\left(\alpha_{3(j+m)}\right)$.

If $2 s \leqslant j<3 s$, then

$$
b_{i j}=\left\{\begin{array}{l}
e_{4(j+m+1)+1} \otimes w_{4 j+1 \rightarrow 4(j+1)}, \quad i=(j+1)_{2 s} \\
w_{4(j+m)+3 \rightarrow 4(j+m+1)+1} \otimes w_{4 j+1 \rightarrow 4 j+2}, \quad i=j+2 s \\
0, \quad \text { otherwise. }
\end{array}\right.
$$

If $3 s \leqslant j<4 s$, then

$$
b_{i j}=\left\{\begin{array}{l}
e_{4(j+m+1)+1} \otimes w_{4 j+1 \rightarrow 4(j+1)}, \quad i=(j+1)_{2 s} \\
0, \quad \text { otherwise. }
\end{array}\right.
$$

If $4 s \leqslant j<5 s$, then $b_{i j}=0$.

If $5 s \leqslant j<6 s$, then

$$
b_{i j}=\left\{\begin{array}{l}
w_{4(j+m)+3 \rightarrow 4(j+m+s+1)+2} \otimes e_{4(j+s)+2}, \quad i=j-s \\
0, \quad \text { otherwise. }
\end{array}\right.
$$

If $6 s \leqslant j<7 s$, then

$$
b_{i j}=\left\{\begin{array}{l}
-w_{4(j+m)+3 \rightarrow 4(j+m+1)+1} \otimes e_{4(j+s)+2}, \quad i=j-s \\
0, \quad \text { otherwise. }
\end{array}\right.
$$


HOCHSCHILD COHOMOLOGY RING FOR SELF-INJECTIVE ALGEBRAS OF TREE CLASS E . II. 139 If $7 s \leqslant j<8 s$, then $b_{i j}=0$.

If $8 s \leqslant j<9 s$, then

$$
b_{i j}=\left\{\begin{array}{l}
\kappa_{1} w_{4(j+m+1)+2 \rightarrow 4(j+m+1)+3} \otimes e_{4 j+3}, \quad i=j-2 s \\
\kappa_{1} w_{4(j+m+1)+1 \rightarrow 4(j+m+1)+3} \otimes w_{4 j+3 \rightarrow 4(j+1)}, \quad i=(j+1)_{s}, \quad j<9 s-1 ; \\
\kappa_{1} w_{4(j+m+s+1)+1 \rightarrow 4(j+m+1)+3} \otimes w_{4 j+3 \rightarrow 4(j+1)}, \quad i=s+(j+1)_{s}, j<9 s-1 ; \\
-\kappa_{1} e_{4(j+m+1)+3} \otimes w_{4 j+3 \rightarrow 4(j+1)+2}, \quad i=4 s+(j+1)_{s}, j<9 s-1 ; \\
-\kappa_{1} e_{4(j+m+1)+3} \otimes w_{4 j+3 \rightarrow 4(j+s+1)+2}, \quad i=5 s+(j+1)_{s}, \quad j<9 s-1 ; \\
0, \quad \text { otherwise, }
\end{array}\right.
$$

where $\kappa_{1}=\kappa^{\ell+1}\left(\alpha_{3(j+m)}\right)$.

(7) If $r_{0}=6$, then $\Omega^{6}\left(Y_{t}^{(22)}\right)$ is described with $(8 s \times 9 s)$-matrix with the following elements $b_{i j}$ : If $0 \leqslant j<2 s$, then

$$
b_{i j}=\left\{\begin{array}{l}
w_{4(j+m) \rightarrow 4(j+m+s)+1} \otimes e_{4 j}, \quad i=(j)_{s} \\
e_{4(j+m+s)+1} \otimes w_{4 j \rightarrow 4(j+s)+1}, \quad i=j+3 s f_{0}(j, s) \\
0, \quad \text { otherwise. }
\end{array}\right.
$$

If $2 s \leqslant j<4 s$, then

$$
b_{i j}=\left\{\begin{array}{l}
\kappa^{\ell+1}\left(\alpha_{3(j+m)}\right) e_{4(j+m+1)} \otimes w_{4 j+1 \rightarrow 4(j+1)}, \quad i=(j+1)_{s} \\
0, \quad \text { otherwise. }
\end{array}\right.
$$

If $4 s \leqslant j<6 s$, then

$$
b_{i j}=\left\{\begin{array}{l}
\kappa_{1} w_{4(j+m)+2 \rightarrow 4(j+m)+3} \otimes e_{4 j+2}, \quad i=j+s \\
-\kappa_{1} e_{4(j+m)+3} \otimes w_{4 j+2 \rightarrow 4 j+3}, \quad i=j+2 s, j \geqslant 5 s \\
0, \quad \text { otherwise, }
\end{array}\right.
$$

where $\kappa_{1}=-\kappa^{\ell+1}\left(\alpha_{3(j+m-1)}\right)$.

If $6 s \leqslant j<7 s$, then $b_{i j}=0$.

If $7 s \leqslant j<8 s$, then

$$
b_{i j}=\left\{\begin{array}{l}
w_{4(j+m)+3 \rightarrow 4(j+m+s+1)+2} \otimes e_{4 j+3}, \quad i=j \\
0, \quad \text { otherwise. }
\end{array}\right.
$$

(8) If $r_{0}=7$, then $\Omega^{7}\left(Y_{t}^{(22)}\right)$ is described with $(9 s \times 8 s)$-matrix with the following elements $b_{i j}$ : 
If $0 \leqslant j<s$, then

$$
b_{i j}=\left\{\begin{array}{l}
\kappa_{1} w_{4(j+m+s)+2 \rightarrow 4(j+m+1)} \otimes e_{4 j}, \quad i=j+s \\
-\kappa_{1} w_{4(j+m)+3 \rightarrow 4(j+m+1)} \otimes w_{4 j \rightarrow 4 j+1}, \quad i=j+2 s \\
\kappa_{1} e_{4(j+m+1)} \otimes w_{4 j \rightarrow 4 j+2}, \quad i=j+4 s \\
\kappa_{1} e_{4(j+m+1)} \otimes w_{4 j \rightarrow 4(j+s)+2}, \quad i=j+5 s \\
0, \quad \text { otherwise }
\end{array}\right.
$$

where $\kappa_{1}=-\kappa^{\ell+1}\left(\alpha_{3(j+m)}\right)$.

If $s \leqslant j<2 s$, then $b_{i j}=0$.

If $2 s \leqslant j<3 s$, then

$$
b_{i j}=\left\{\begin{array}{l}
-e_{4(j+m+1)+2} \otimes w_{4 j+1 \rightarrow 4(j+1)}, \quad i=(j+1)_{2 s} \\
-w_{4(j+m)+3 \rightarrow 4(j+m+1)+2} \otimes e_{4 j+1}, \quad i=j \\
0, \quad \text { otherwise. }
\end{array}\right.
$$

If $3 s \leqslant j<4 s$, then $b_{i j}=0$.

If $4 s \leqslant j<5 s$, then

$$
b_{i j}=\left\{\begin{array}{l}
-e_{4(j+m+s+1)+2} \otimes w_{4(j+s)+1 \rightarrow 4(j+1)}, \quad i=(j+s+1)_{2 s} \\
-w_{4(j+m)+3 \rightarrow 4(j+m+s+1)+2} \otimes e_{4(j+s)+1}, \quad i=j-s \\
0, \quad \text { otherwise. }
\end{array}\right.
$$

If $5 s \leqslant j<7 s$, then $b_{i j}=0$.

If $7 s \leqslant j<8 s$, then

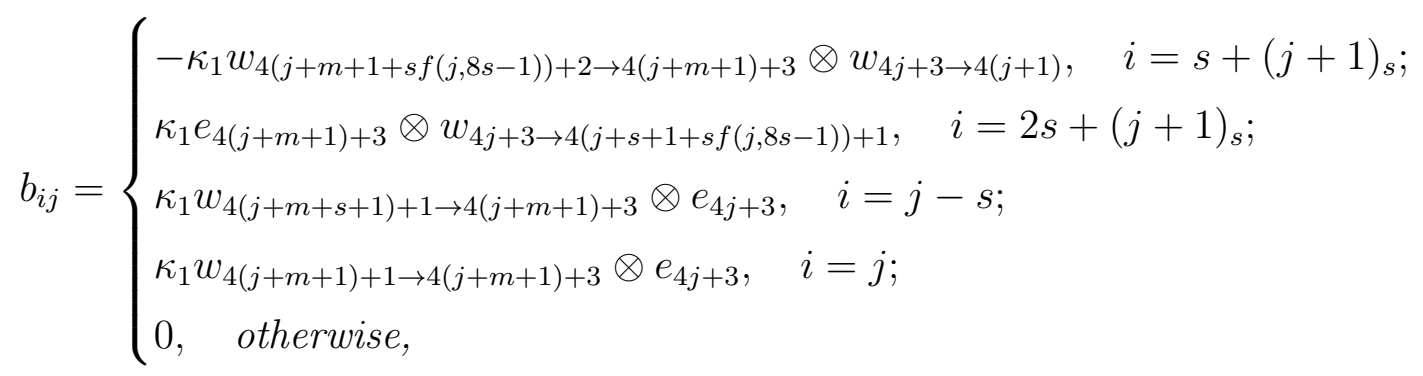

where $\kappa_{1}=-\kappa^{\ell+1}\left(\alpha_{3(j+m)}\right)$.

If $8 s \leqslant j<9 s$, then $b_{i j}=0$.

(9) If $r_{0}=8$, then $\Omega^{8}\left(Y_{t}^{(22)}\right)$ is described with $(8 s \times 6 s)$-matrix with the following elements $b_{i j}$ : If $0 \leqslant j<2 s$, then

$$
b_{i j}=\left\{\begin{array}{l}
w_{4(j+m)-1 \rightarrow 4(j+m+s)+2} \otimes e_{4 j}, \quad i=j, \quad j<s \\
-e_{4(j+m+s)+2} \otimes w_{4 j \rightarrow 4(j+s)+1}, \quad i=s+(j+s)_{2 s} \\
-w_{4(j+m+s)+1 \rightarrow 4(j+m+s)+2} \otimes w_{4 j \rightarrow 4 j+2}, \quad i=j+3 s \\
0, \quad \text { otherwise. }
\end{array}\right.
$$


HOCHSCHILD COHOMOLOGY RING FOR SELF-INJECTIVE ALGEBRAS OF TREE CLASS $E_{6}$. II. 141 If $2 s \leqslant j<4 s$, then

$$
b_{i j}=\left\{\begin{array}{l}
-\kappa_{1} w_{4(j+m)+2 \rightarrow 4(j+m)+3} \otimes e_{4 j+1}, \quad i=j-s \\
\kappa_{1} w_{4(j+m+s)+1 \rightarrow 4(j+m)+3} \otimes w_{4 j+1 \rightarrow 4 j+2}, \quad i=j+s \\
\kappa_{1} e_{4(j+m)+3} \otimes w_{4 j+1 \rightarrow 4(j+1)}, \quad i=(j+1)_{s}, \quad j<3 s-1 \text { or } j=4 s-1 \\
0, \quad \text { otherwise }
\end{array}\right.
$$

where $\kappa_{1}=-\kappa^{\ell+1}\left(\alpha_{3(j+m-1)}\right)$.

If $4 s \leqslant j<5 s$, then $b_{i j}=0$.

If $5 s \leqslant j<6 s$, then

$$
b_{i j}=\left\{\begin{array}{l}
\kappa^{\ell+1}\left(\alpha_{3(j+m)}\right) e_{4(j+m+1)} \otimes w_{4 j+2 \rightarrow 4 j+3}, \quad i=j \\
0, \quad \text { otherwise. }
\end{array}\right.
$$

If $6 s \leqslant j<7 s$, then

$$
b_{i j}=\left\{\begin{array}{l}
-w_{4(j+m+1) \rightarrow 4(j+m+s+1)+1} \otimes e_{4 j+3}, \quad i=j-s \\
0, \quad \text { otherwise }
\end{array}\right.
$$

If $7 s \leqslant j<8 s$, then $b_{i j}=0$.

(10) If $r_{0}=9$, then $\Omega^{9}\left(Y_{t}^{(22)}\right)$ is described with $(6 s \times 7 s)$-matrix with the following elements $b_{i j}$ :

If $0 \leqslant j<s$, then

$$
b_{i j}=\left\{\begin{array}{l}
\kappa^{\ell+1}\left(\alpha_{3(j+m-1)}\right) e_{4(j+m)+3} \otimes e_{4 j}, \quad i=j \\
0, \quad \text { otherwise }
\end{array}\right.
$$

If $s \leqslant j<3 s$, then

$$
b_{i j}=\left\{\begin{array}{l}
w_{4(j+m+1) \rightarrow 4(j+m+1)+2} \otimes e_{4(j+s)+1}, \quad i=j \\
0, \quad \text { otherwise. }
\end{array}\right.
$$

If $3 s \leqslant j<5 s$, then $b_{i j}=0$.

If $5 s \leqslant j<6 s$, then

$$
b_{i j}=\left\{\begin{array}{l}
\kappa_{1} e_{4(j+m+2)} \otimes w_{4 j+3 \rightarrow 4(j+s+1+s f(j, 6 s-1))+1}, \quad i=s+(j+1)_{s} \\
\kappa_{1} w_{4(j+m+s+1)+2 \rightarrow 4(j+m+2)} \otimes e_{4 j+3}, \quad i=j ; \\
-\kappa_{1} w_{4(j+m+1)+3 \rightarrow 4(j+m+2)} \otimes w_{4 j+3 \rightarrow 4(j+1)}, \quad i=(j+1)_{s}, j=6 s-1 \\
0, \quad \text { otherwise, }
\end{array}\right.
$$

where $\kappa_{1}=-\kappa^{\ell+1}\left(\alpha_{3(j+m+1)}\right)$. $b_{i j}$ :

(11) If $r_{0}=10$, then $\Omega^{10}\left(Y_{t}^{(22)}\right)$ is described with $(7 s \times 6 s)$-matrix with the following elements 
If $0 \leqslant j<s$, then

$$
b_{i j}=\left\{\begin{array}{l}
-\kappa_{1} w_{4(j+m) \rightarrow 4(j+m)+3} \otimes e_{4 j}, \quad i=j ; \\
-\kappa_{1} w_{4(j+m+s)+1 \rightarrow 4(j+m)+3} \otimes w_{4 j \rightarrow 4 j+1}, \quad i=j+s ; \\
\kappa_{1} w_{4(j+m)+1 \rightarrow 4(j+m)+3} \otimes w_{4 j \rightarrow 4(j+s)+1}, \quad i=j+2 s ; \\
\kappa_{1} w_{4(j+m+s)+2 \rightarrow 4(j+m)+3} \otimes w_{4 j \rightarrow 4 j+2}, \quad i=j+3 s ; \\
-\kappa_{1} w_{4(j+m)+2 \rightarrow 4(j+m)+3} \otimes w_{4 j \rightarrow 4(j+s)+2}, \quad i=j+4 s ; \\
\kappa_{1} e_{4(j+m)+3} \otimes w_{4 j \rightarrow 4 j+3}, \quad i=j+5 s ; \\
0, \quad \text { otherwise, }
\end{array}\right.
$$

where $\kappa_{1}=-\kappa^{\ell+1}\left(\alpha_{3(j+m-1)}\right)$.

If $s \leqslant j<3 s$, then

$$
b_{i j}=\left\{\begin{array}{l}
-\kappa_{1} w_{4(j+m)+1 \rightarrow 4(j+m+1)} \otimes e_{4(j+s)+1}, \quad i=j ; \\
\kappa_{1} w_{4(j+m)+2 \rightarrow 4(j+m+1)} \otimes w_{4(j+s)+1 \rightarrow 4(j+s)+2}, \quad i=j+2 s ; \\
\kappa_{1} w_{4(j+m)+3 \rightarrow 4(j+m+1)} \otimes w_{4(j+s)+1 \rightarrow 4 j+3}, \quad i=j+4 s, j<2 s ; \\
\kappa_{1} e_{4(j+m+1)} \otimes w_{4(j+s)+1 \rightarrow 4(j+1)}, \quad i=(j+1)_{s}, j<2 s-1 \text { or } j=3 s-1 ; \\
0, \quad \text { otherwise, }
\end{array}\right.
$$

where $\kappa_{1}=-\kappa^{\ell+1}\left(\alpha_{3(j+m)}\right)$.

If $3 s \leqslant j<4 s$, then

$$
b_{i j}= \begin{cases}w_{4(j+m+1) \rightarrow 4(j+m+s+1)+1} \otimes w_{4(j+s)+2 \rightarrow 4(j+1)}, & i=(j+1)_{s}, j<4 s-1 ; \\ w_{4(j+m)+3 \rightarrow 4(j+m+s+1)+1} \otimes w_{4(j+s)+2 \rightarrow 4 j+3}, & i=j+2 s ; \\ 0, \quad \text { otherwise. } & \end{cases}
$$

If $4 s \leqslant j<5 s-1$, then $b_{i j}=0$.

If $5 s-1 \leqslant j<5 s$, then

$$
b_{i j}=\left\{\begin{array}{l}
w_{4(j+m+1) \rightarrow 4(j+m+s+1)+1} \otimes w_{4(j+s)+2 \rightarrow 4(j+1)}, \quad i=(j+1)_{s} \\
0, \quad \text { otherwise. }
\end{array}\right.
$$

If $5 s \leqslant j<6 s-1$, then $b_{i j}=0$.

If $6 s-1 \leqslant j<6 s$, then

$$
b_{i j}=\left\{\begin{array}{l}
-w_{4(j+m+1) \rightarrow 4(j+m+1)+2} \otimes w_{4 j+3 \rightarrow 4(j+1)}, \quad i=(j+1)_{s} \\
0, \quad \text { otherwise. }
\end{array}\right.
$$

If $6 s \leqslant j<7 s$, then

$$
b_{i j}=\left\{\begin{array}{l}
-w_{4(j+m)+3 \rightarrow 4(j+m+1)+2} \otimes e_{4 j+3}, \quad i=j-s \\
-w_{4(j+m+1) \rightarrow 4(j+m+1)+2} \otimes w_{4 j+3 \rightarrow 4(j+1)}, \quad i=(j+1)_{s}, j<7 s-1 \\
0, \quad \text { otherwise. }
\end{array}\right.
$$


HOCHSCHILD COHOMOLOGY RING FOR SELF-INJECTIVE ALGEBRAS OF TREE CLASS $E_{6}$. II. 143

(II) Represent an arbitrary $t_{0} \in \mathbb{N}$ in the form $t_{0}=11 \ell_{0}+r_{0}$, where $0 \leqslant r_{0} \leqslant 10$. Then $\Omega^{t_{0}}\left(Y_{t}^{(22)}\right)$ is a $\Omega^{r_{0}}\left(Y_{t}^{(22)}\right)$, whose left components twisted by $\sigma^{\ell_{0}}$, and coefficients multiplied by $(-1)^{\ell_{0}}$.

Proposition 39 (Translates for the case 23). (I) Let $r_{0} \in \mathbb{N}, r_{0}<11$. $r_{0}$-translates of the elements $Y_{t}^{(23)}$ are described by the following way.

(1) If $r_{0}=0$, then $\Omega^{0}\left(Y_{t}^{(23)}\right)$ is described with $(6 s \times 6 s)$-matrix with one nonzero element that is of the following form:

$$
b_{j, 0}=w_{4(j+m) \rightarrow 4(j+m+1)} \otimes e_{4 j} .
$$

(2) If $r_{0}=1$, then $\Omega^{1}\left(Y_{t}^{(23)}\right)$ is described with $(7 s \times 7 s)$-matrix with one nonzero element that is of the following form:

$$
b_{j, 6 s}=w_{4(j+m+1) \rightarrow 4(j+m+2)} \otimes e_{4 j+3} .
$$

(3) If $r_{0}=2$, then $\Omega^{2}\left(Y_{t}^{(23)}\right)$ is described with $(6 s \times 6 s)$-matrix with one nonzero element that is of the following form:

$$
b_{j, 5 s}=w_{4(j+m+1) \rightarrow 4(j+m+2)} \otimes e_{4 j+3} .
$$

(4) If $r_{0}=3$, then $\Omega^{3}\left(Y_{t}^{(23)}\right)$ is described with $(8 s \times 8 s)$-matrix with the following two nonzero elements:

$$
\begin{aligned}
& b_{j, 4 s}=w_{4(j+m+1) \rightarrow 4(j+m+2)} \otimes e_{4 j+2} ; \\
& b_{j, 5 s}=w_{4(j+m+1) \rightarrow 4(j+m+2)} \otimes e_{4 j+2} .
\end{aligned}
$$

(5) If $r_{0}=4$, then $\Omega^{4}\left(Y_{t}^{(23)}\right)$ is described with $(9 s \times 9 s)$-matrix with one nonzero element that is of the following form:

$$
b_{j, s}=w_{4(j+m) \rightarrow 4(j+m+1)} \otimes e_{4 j} .
$$

(6) If $r_{0}=5$, then $\Omega^{5}\left(Y_{t}^{(23)}\right)$ is described with $(8 s \times 8 s)$-matrix with the following two nonzero elements:

$$
\begin{aligned}
& b_{j, 2 s}=w_{4(j+m+1) \rightarrow 4(j+m+2)} \otimes e_{4 j+1} ; \\
& b_{j, 3 s}=w_{4(j+m+1) \rightarrow 4(j+m+2)} \otimes e_{4 j+1} .
\end{aligned}
$$

(7) If $r_{0}=6$, then $\Omega^{6}\left(Y_{t}^{(23)}\right)$ is described with $(9 s \times 9 s)$-matrix with the following two nonzero elements:

$$
\begin{gathered}
b_{j, 0}=w_{4(j+m) \rightarrow 4(j+m+1)} \otimes e_{4 j} ; \\
b_{j, 8 s}=w_{4(j+m+1) \rightarrow 4(j+m+2)} \otimes e_{4 j+3} .
\end{gathered}
$$

(8) If $r_{0}=7$, then $\Omega^{7}\left(Y_{t}^{(23)}\right)$ is described with $(8 s \times 8 s)$-matrix with the following two nonzero elements:

$$
\begin{aligned}
& b_{j, 4 s}=w_{4(j+m+1) \rightarrow 4(j+m+2)} \otimes e_{4 j+2} ; \\
& b_{j, 5 s}=w_{4(j+m+1) \rightarrow 4(j+m+2)} \otimes e_{4 j+2} .
\end{aligned}
$$

(9) If $r_{0}=8$, then $\Omega^{8}\left(Y_{t}^{(23)}\right)$ is described with $(6 s \times 6 s)$-matrix with one nonzero element that is of the following form:

$$
b_{j, 5 s}=w_{4(j+m+1) \rightarrow 4(j+m+2)} \otimes e_{4 j+3} .
$$

(10) If $r_{0}=9$, then $\Omega^{9}\left(Y_{t}^{(23)}\right)$ is described with $(7 s \times 7 s)$-matrix with the following two nonzero elements:

$$
\begin{aligned}
b_{j, s} & =w_{4(j+m+1) \rightarrow 4(j+m+2)} \otimes e_{4(j+s)+1} ; \\
b_{j, 2 s} & =w_{4(j+m+1) \rightarrow 4(j+m+2)} \otimes e_{4(j+s)+1} .
\end{aligned}
$$


(11) If $r_{0}=10$, then $\Omega^{10}\left(Y_{t}^{(23)}\right)$ is described with $(6 s \times 6 s)$-matrix with one nonzero element that is of the following form:

$$
b_{j, 0}=w_{4(j+m) \rightarrow 4(j+m+1)} \otimes e_{4 j} .
$$

(II) Represent an arbitrary $t_{0} \in \mathbb{N}$ in the form $t_{0}=11 \ell_{0}+r_{0}$, where $0 \leqslant r_{0} \leqslant 10$. Then $\Omega^{t_{0}}\left(Y_{t}^{(23)}\right)$ is a $\Omega^{r_{0}}\left(Y_{t}^{(23)}\right)$, whose left components twisted by $\sigma^{\ell_{0}}$.

Proposition 40 (Translates for the case 24). (I) Let $r_{0} \in \mathbb{N}, r_{0}<11$. $r_{0}$-translates of the elements $Y_{t}^{(24)}$ are described by the following way.

(1) If $r_{0}=0$, then $\Omega^{0}\left(Y_{t}^{(24)}\right)$ is described with $(6 s \times 6 s)$-matrix with one nonzero element that is of the following form:

$$
b_{j, 5}=w_{4 j+3 \rightarrow 4(j+1)+3} \otimes e_{4 j+3} .
$$

(2) If $r_{0}=1$, then $\Omega^{1}\left(Y_{t}^{(24)}\right)$ is described with $(7 s \times 7 s)$-matrix with the following two nonzero elements:

$$
\begin{aligned}
& b_{j, 4 s}=w_{4(j+m)+3 \rightarrow 4(j+m+1)+3} \otimes e_{4 j+2} \\
& b_{j, 5 s}=w_{4(j+m)+3 \rightarrow 4(j+m+1)+3} \otimes e_{4 j+2} .
\end{aligned}
$$

(3) If $r_{0}=2$, then $\Omega^{2}\left(Y_{t}^{(24)}\right)$ is described with $(6 s \times 6 s)$-matrix with one nonzero element that is of the following form:

$$
b_{j, 0}=w_{4(j+m)+3 \rightarrow 4(j+m+1)+3} \otimes e_{4 j} .
$$

(4) If $r_{0}=3$, then $\Omega^{3}\left(Y_{t}^{(24)}\right)$ is described with $(8 s \times 8 s)$-matrix with the following two nonzero elements:

$$
\begin{gathered}
b_{j-2 s, 2 s}=w_{4(j+m)+2 \rightarrow 4(j+m)+3} \otimes w_{4 j+1 \rightarrow 4 j} ; \\
b_{j-2 s, 3 s}=-w_{4(j+m)+2 \rightarrow 4(j+m)+3} \otimes w_{4 j+1 \rightarrow 4 j} .
\end{gathered}
$$

(5) If $r_{0}=4$, then $\Omega^{4}\left(Y_{t}^{(24)}\right)$ is described with $(9 s \times 9 s)$-matrix with the following two nonzero elements:

$$
\begin{gathered}
b_{j, 0}=e_{4(j+m)+3} \otimes w_{4 j \rightarrow 4(j+1)} ; \\
b_{j, s}=e_{4(j+m)} \otimes w_{4 j \rightarrow 4(j+1)} .
\end{gathered}
$$

(6) If $r_{0}=5$, then $\Omega^{5}\left(Y_{t}^{(24)}\right)$ is described with $(8 s \times 8 s)$-matrix with the following two nonzero elements:

$$
\begin{aligned}
& b_{j, 0}=e_{4(j+m)+1} \otimes w_{4 j \rightarrow 4(j+1)} ; \\
& b_{j, s}=e_{4(j+m)+1} \otimes w_{4 j \rightarrow 4(j+1)} .
\end{aligned}
$$

(7) If $r_{0}=6$, then $\Omega^{6}\left(Y_{t}^{(24)}\right)$ is described with $(9 s \times 9 s)$-matrix with one nonzero element that is of the following form:

$$
b_{j, 0}=e_{4(j+m)} \otimes w_{4 j \rightarrow 4(j+1)} .
$$

(8) If $r_{0}=7$, then $\Omega^{7}\left(Y_{t}^{(24)}\right)$ is described with $(8 s \times 8 s)$-matrix with the following two nonzero elements:

$$
\begin{aligned}
& b_{j, 0}=e_{4(j+m)+2} \otimes w_{4 j \rightarrow 4(j+1)} \\
& b_{j, s}=e_{4(j+m)+2} \otimes w_{4 j \rightarrow 4(j+1)} .
\end{aligned}
$$

(9) If $r_{0}=8$, then $\Omega^{8}\left(Y_{t}^{(24)}\right)$ is described with $(6 s \times 6 s)$-matrix with one nonzero element that is of the following form:

$$
b_{j, 0}=e_{4(j+m)+3} \otimes w_{4 j \rightarrow 4(j+1)} .
$$


HOCHSCHILD COHOMOLOGY RING FOR SELF-INJECTIVE ALGEBRAS OF TREE CLASS $E_{6}$. II. 145

(10) If $r_{0}=9$, then $\Omega^{9}\left(Y_{t}^{(24)}\right)$ is described with $(7 s \times 7 s)$-matrix with the following two nonzero elements:

$$
\begin{aligned}
b_{j+s, 0} & =-w_{4(j+m) \rightarrow 4(j+m)+3} \otimes w_{4 j \rightarrow 4 j+1} ; \\
b_{j+2 s, 0} & =w_{4(j+m) \rightarrow 4(j+m)+3} \otimes w_{4 j \rightarrow 4(j+1)+1} .
\end{aligned}
$$

(11) If $r_{0}=10$, then $\Omega^{10}\left(Y_{t}^{(24)}\right)$ is described with $(6 s \times 6 s)$-matrix with the following nonzero elements:

$$
\begin{gathered}
b_{j, 0}=-w_{4(j+m) \rightarrow 4(j+m+1)} \otimes e_{4 j} ; \\
b_{j-5 s, 5 s}=w_{4(j+m) \rightarrow 4(j+m)+3} \otimes w_{4 j+3 \rightarrow 4 j} ; \\
b_{j, 5 s}=w_{4(j+m)+3 \rightarrow 4(j+m+1)+3} \otimes e_{4 j+3} .
\end{gathered}
$$

(II) Represent an arbitrary $t_{0} \in \mathbb{N}$ in the form $t_{0}=11 \ell_{0}+r_{0}$, where $0 \leqslant r_{0} \leqslant 10$. Then $\Omega^{t_{0}}\left(Y_{t}^{(24)}\right)$ is a $\Omega^{r_{0}}\left(Y_{t}^{(24)}\right)$, whose left components twisted by $\sigma^{\ell_{0}}$.

\section{Multiplications in $\mathrm{HH}^{*}(R)$}

From the descriptions of elements $Y_{t}^{(i)}$ and its $\Omega$-translates we can find multiplications of the elements using the formula (囝).

We will find a multiplication of elements of the types 4 and 3 for $s>1$.

Consider two arbitrary elements $Y_{t_{4}}^{(4)}$ and $Y_{t_{3}}^{(3)}$. For its degrees $t_{4}$ and $t_{3}$ we have:

$$
\begin{aligned}
& t_{4}=11 \ell_{4}+1, \ell_{4}(n+s) \equiv s(2 s), \ell_{4} \% 2 \text { or char } K=2 ; \\
& t_{3}=11 \ell_{3}+1, \ell_{3}(n+s) \equiv 0(2 s), \ell_{3} \vdots 2 \text { or char } K=2 .
\end{aligned}
$$

Let $t=t_{4}+t_{3}$; this is the degree of an element $Y_{t_{4}}^{(4)} Y_{t_{3}}^{(3)}$. Then $t=11\left(\ell_{4}+\ell_{3}\right)+2$. Group of the degree $t$ has type (5). $Y_{t}^{(3)}$ is an $(7 s \times 6 s)$-matrix with two nonzero elements $y_{0,0}=w_{0 \rightarrow 1} \otimes e_{0}$ and $y_{0, s}=w_{0 \rightarrow 4 s+1} \otimes e_{0} . \Omega^{t_{3}}\left(Y_{t_{4}}^{(4)}\right)$ is an $(6 s \times 7 s)$-matrix that was described in proposition 20 . Multiplication of $\Omega^{t_{3}}\left(Y_{t_{4}}^{(4)}\right)$ and $Y_{t}^{(3)}$ an $(6 s \times 6 s)$-matrix with the following two nonzero elements:

$$
\begin{gathered}
b_{0,0}=\kappa^{\ell_{4}}\left(\alpha_{0}\right) \kappa^{\ell_{3}}\left(\alpha_{1}\right) \kappa^{\ell_{3}}\left(\alpha_{2}\right) w_{0 \rightarrow 3} \otimes e_{0} ; \\
b_{0,3 s-1}=-\kappa^{\ell_{3}}\left(\alpha_{3 s-1}\right) w_{0 \rightarrow 4 s+2} \otimes w_{8 s-3 \rightarrow 0} .
\end{gathered}
$$

We have $\ell_{3}: 2$, hence $\kappa^{\ell_{3}}\left(\alpha_{1}\right)=\kappa^{\ell_{3}}\left(\alpha_{3 s-1}\right)=1, \kappa^{\ell_{3}}\left(\alpha_{2}\right)=\kappa^{\ell_{3}}\left(\alpha_{0}\right), \kappa^{\ell_{4}}\left(\alpha_{0}\right) \kappa^{\ell_{3}}\left(\alpha_{1}\right) \kappa^{\ell_{3}}\left(\alpha_{2}\right)=$ $\kappa^{\ell_{4}}\left(\alpha_{0}\right) \kappa^{\ell_{3}}\left(\alpha_{0}\right)=\kappa^{\ell_{4}+\ell_{3}}\left(\alpha_{0}\right) . Y_{t}^{(5)}$ is an $(6 s \times 6 s)$-matrix with a single nonzero element $y_{0,0}=$ $\kappa^{\ell}\left(\alpha_{0}\right) w_{0 \rightarrow 3} \otimes e_{0}$, hence $Y^{(4)} Y^{(3)}$ coincide with $Y^{(5)}$ for degree of type (5).

Multiplications of other elements, except $Y^{(5)}, Y^{(10)}, Y^{(17)}, Y^{(19)}$ and $Y^{(21)}$, are similarly considered. To get the whole picture we should prove the following lemma.

\section{Lemma 41.}

(a) Let $Y^{(5)}$ be an arbitrary element from generators of the corresponding type. Then there are elements $Y^{(3)}$ and $Y^{(4)}$ such as $Y^{(5)}=Y^{(3)} Y^{(4)}$.

(b) Let $Y^{(10)}$ be an arbitrary element from generators of the corresponding type. Then there are elements $Y^{(3)}$ and $Y^{(6)}$ such as $Y^{(10)}=Y^{(3)} Y^{(6)}$.

(c) Let $Y^{(17)}$ be an arbitrary element from generators of the corresponding type. Then there are elements $Y^{(3)}$ and $Y^{(15)}$ such as $Y^{(17)}=Y^{(3)} Y^{(15)}$.

(d) Let $Y^{(19)}$ be an arbitrary element from generators of the corresponding type. Then there are elements $Y^{(3)}$ and $Y^{(18)}$ such as $Y^{(19)}=Y^{(3)} Y^{(18)}$. 
(e) Let $Y^{(21)}$ be an arbitrary element from generators of the corresponding type. Then there are elements $Y^{(3)}$ and $Y^{(20)}$ such as $Y^{(21)}=Y^{(3)} Y^{(20)}$.

Proof. The degree 1 has type 3, for all $s$. It only remains to use the relations for type (3).

\section{REFERENCES}

[1] C. Riedtmann, Algebren, Darstellungsköcher, Überlagerungen und zurück. - Comment. Math. Helv., 1980, v. 55, 199-224.

[2] K. Erdmann, T. Holm, Twisted bimodules and Hochschild cohomology for self-injective algebras of class $A_{n}$. — Forum Math., 1999, v. 11, 177-201.

[3] A. I. Generalov, M. A. Kachalova, Bimodule Resolution of Möbius Algebras. — Zap. Nauchn. Semin. POMI, 321 (2005), 36-66.

[4] M. A. Kachalova, Hochschild cohomology of Möbius Algebras. — Zap. Nauchn. Semin. POMI, 330 (2006), 173-200.

[5] M. A. Pustovykh, Hochschild cohomology ring of Möbius algebras. — Zap. Nauchn. Semin. POMI, 388 (2011), 210-246.

[6] Yu. V. Volkov, A. I. Generalov, Hochschild cohomology for self-injective algebras of tree class $D_{n}$. I. — Zap. Nauchn. Semin. POMI, 343 (2007), 121-182.

[7] Yu. V. Volkov, Hochschild cohomology for self-injective algebras of tree class $D_{n}$. II. — Zap. Nauchn. Semin. POMI, 365 (2009), 63-121.

[8] Yu. V. Volkov, A. I. Generalov, Hochschild cohomology for self-injective algebras of tree class $D_{n}$. III. Zap. Nauchn. Semin. POMI, 386 (2011), 100-128.

[9] Yu. V. Volkov, Hochschild cohomology for nonstandard self-injective algebras of tree class $D_{n}$. - Zap. Nauchn. Semin. POMI, 388 (2011), 48-99.

[10] Yu. V. Volkov, Hochschild cohomology for self-injective algebras of tree class $D_{n}$. IV. — Zap. Nauchn. Semin. POMI, 388 (2011), 100-118.

[11] Yu. V. Volkov, Hochschild cohomology for self-injective algebras of tree class $D_{n}$. V. — Zap. Nauchn. Semin. POMI, 394 (2011), 140-173.

[12] M. A. Pustovykh, Hochschild cohomology ring for algebras of type $E_{6}$. — Zap. Nauchn. Semin. POMI, 423 (2014), 205-243.

[13] D. Happel, Hochschild cohomology of finite-dimensional algebras. — Lect. Notes Math., 1989, 1404, 108-126.

[14] Yu. V. Volkov, A. I. Generalov, S. O. Ivanov, On construction of bimodule resolutions with the help of Happel's lemma. — Zap. Nauchn. Semin. POMI, 375 (2010), 61-70.

E-mail address: mashakachalova@mail.ru 